Portland State University

PDXScholar

Summer 6-4-2015

\title{
Intrapersonal and Interpersonal Consequences of Loneliness: Health Behavior, Social Interactions, Self- Disclosure, and Perceived Responsiveness
}

Sarah Noel Arpin

Portland State University

Follow this and additional works at: https://pdxscholar.library.pdx.edu/open_access_etds

Part of the Health Psychology Commons, and the Social Psychology Commons Let us know how access to this document benefits you.

\section{Recommended Citation}

Arpin, Sarah Noel, "Intrapersonal and Interpersonal Consequences of Loneliness: Health Behavior, Social Interactions, Self-Disclosure, and Perceived Responsiveness" (2015). Dissertations and Theses. Paper 2340.

https://doi.org/10.15760/etd.2337

This Dissertation is brought to you for free and open access. It has been accepted for inclusion in Dissertations and Theses by an authorized administrator of PDXScholar. For more information, please contact pdxscholar@pdx.edu. 
Intrapersonal and Interpersonal Consequences of Loneliness: Health Behavior, Social Interactions, Self-Disclosure, and Perceived Responsiveness

by

Sarah Noel Arpin

A dissertation submitted in partial fulfillment of the requirements for the degree of

\author{
Doctor of Philosophy \\ in \\ Applied Psychology
}

\author{
Dissertation Committee: \\ Cynthia D. Mohr, Chair \\ Kimberly Kahn \\ Todd Bodner \\ Jeffrey D. Robinson
}

Portland State University 
(C) 2015 Sarah Noel Arpin 


\begin{abstract}
As a social species, human beings are driven by an innate desire to belong and are thus motivated to develop and maintain meaningful social relationships. As such, perceiving a lack of belongingness strongly impacts psychological and physiological health and wellbeing. A common form of perceived relationship deficits is loneliness, a negativeaffective experience detrimental to health and well-being over time. Through a series of three manuscripts, this dissertation applies the full-cycle model of social psychological research to explore various affective, behavioral, and cognitive consequences of loneliness. Whereas existing models of loneliness focus on long-lasting or chronic forms of loneliness, these studies investigate chronic and transient loneliness, as well as processes through which transient loneliness may develop into more chronic levels. The first paper demonstrates that solitary consumption is a unique behavioral response to transient loneliness, which may exacerbate the experience of loneliness and negatively impact health over time. The second paper provides support for a positivity-deficit perspective, demonstrating that chronic loneliness is related to less disclosure of recent positive experiences, a deficit which may be consequential for the development of close relationships. The third paper demonstrates the role of transient loneliness in inhibiting individuals and their interaction partners from reaping the social rewards of positiveevent disclosure, particularly among those who typically feel socially connected. Taken together, these studies expand the current understanding transient loneliness, revealing various social-cognitive and behavioral consequences which could impede the socialreaffiliation process, and thus contribute to the maintenance of loneliness over time.
\end{abstract}




\section{DEDICATION}

This dissertation is dedicated to all of the support resources and social connections present throughout life - who have made my experiences of loneliness few and far between, and who have provided me comfort in times of stress and company with whom to celebrate times of joy. 


\section{ACKNOWLEDGEMENTS}

I would like to extend my appreciation to my dissertation committee for their support and enthusiasm throughout this process. First, I would like to thank my doctoral advisor, Dr. Cynthia Mohr, for her kindness, support, and encouragement over the past five years. Cynthia, in you I have found not only a lifelong colleague but also a friend. I also wish to thank Dr. Jeffrey Robinson for his generosity in providing me access to his research laboratory as I conducted study three of this dissertation.

I would also like to extend my gratitude to my lab team, who devoted innumerable hours of their time during data collection. Kendra, Jason, Tara, Bitsie, Nina, Yumi, Devin, Martin, Tiffani, Sarah, Megan, and Stephanie, I am immensely grateful for your enthusiasm and dedication to this project. Further, I would like to acknowledge the American Psychological Association for funding my third study through the APA Dissertation Research Award.

I also wish to thank my friends, peers, and colleagues. I feel blessed for your unwavering support and encouragement throughout graduate school.

Last but certainly not least, I would like to extend my gratitude to my parents (Greg and Lori), sister (Megan), and partner (Ian) for their ongoing support, encouragement, and patience over the past five years. This would not have been possible without all of you, and for that I am forever grateful.

I am not afraid of storms, for I am learning how to sail my ship. -Louisa May Alcott 
Table of Contents

Abstract

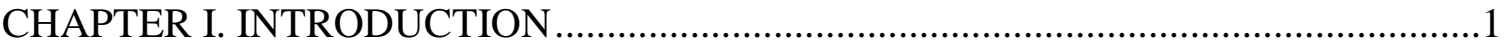

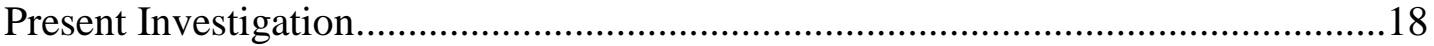

Chapter II Overview: Having friends and feeling lonely: A daily-process examination of transient loneliness, socialization, and drinking behavior .................18

Chapter III Overview: No one to turn to in times of joy: Loneliness and

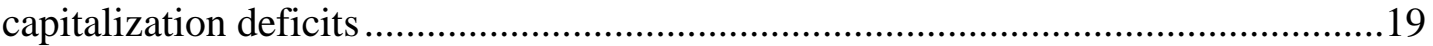

Chapter IV Overview: Experimental induction of loneliness and the perceived provision and receipt of responsive behavior during positive-event disclosure .........21

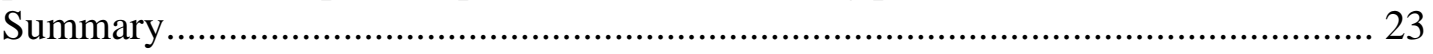

CHAPTER II. MANUSCRIPT I. HAVING FRIENDS AND FEELING LONELY: A DAILY-PROCESS EXAMINATION OF TRANSIENT LONELINESS,

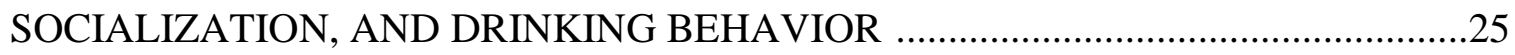

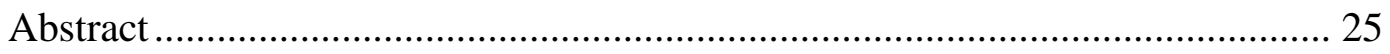

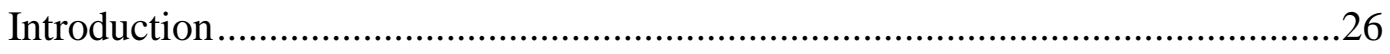

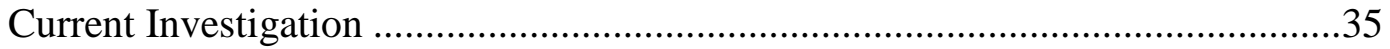

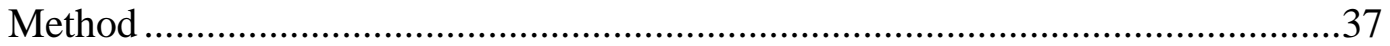

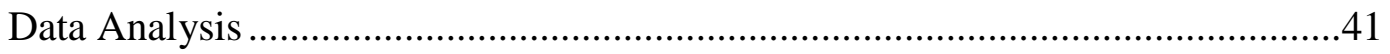

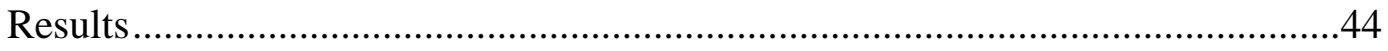

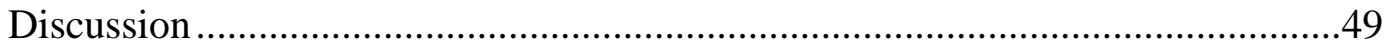

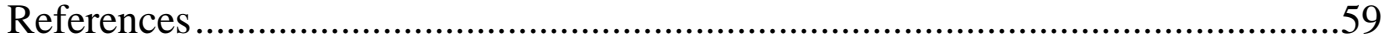

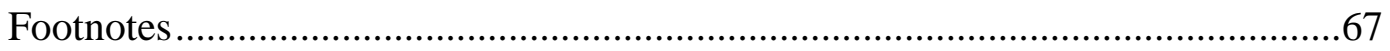

CHAPTER III. MANUSCRIPT II. NO ONE TO TURN TO IN TIMES OF JOY:

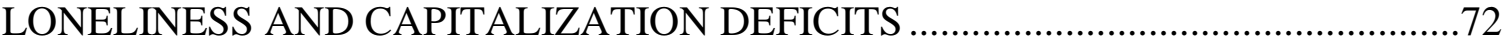

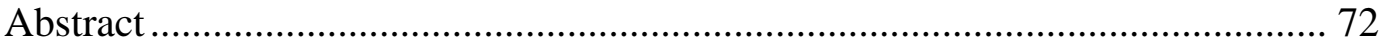

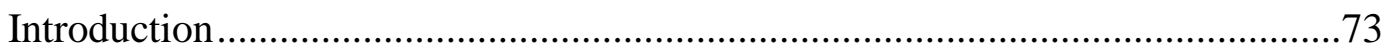

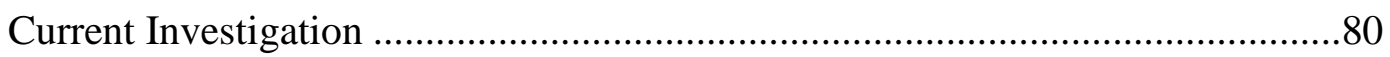

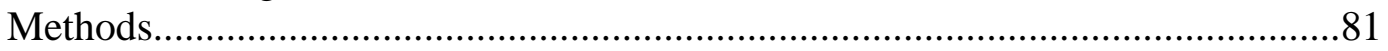

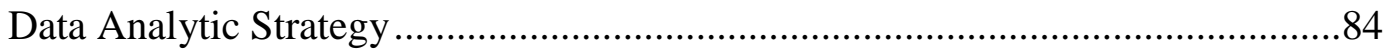

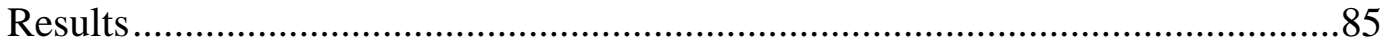

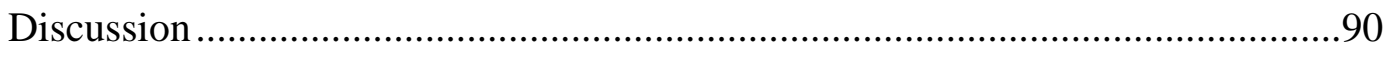

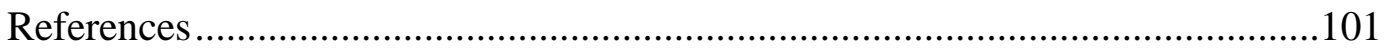

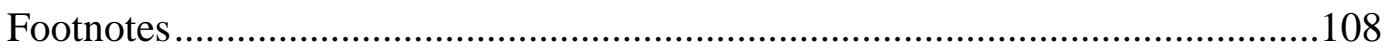


CHAPTER IV. MANUSCRIPT III: EXPERIMENTAL INDUCTION OF LONELINESS AND THE PERCEIVED PROVISION AND RECEIVE OF RESPONSIVE BEHAVIOR DURING POSITIVE-EVENT DISCLOSURE ............................................................117

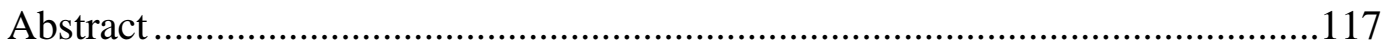

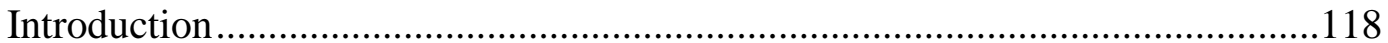

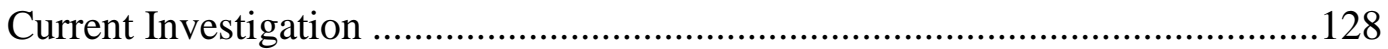

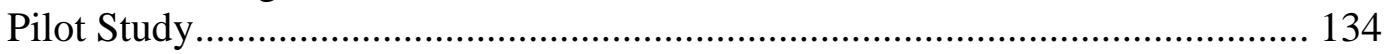

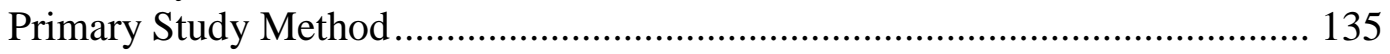

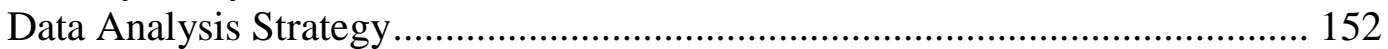

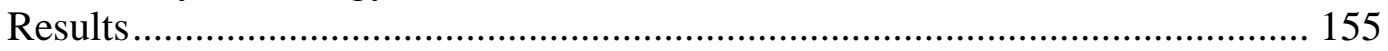

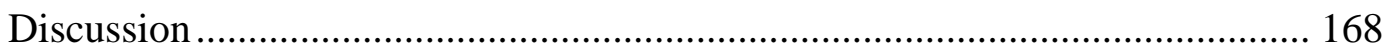

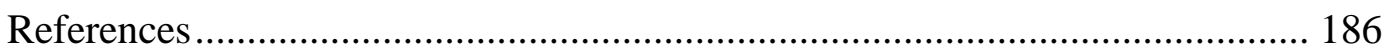

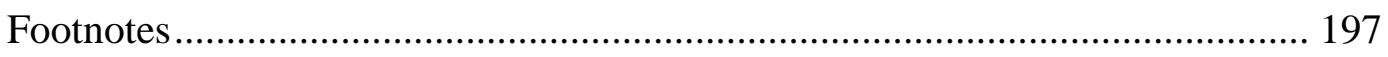

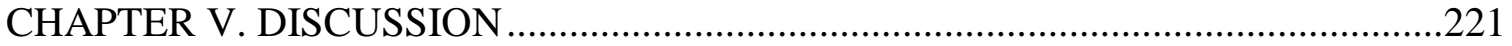

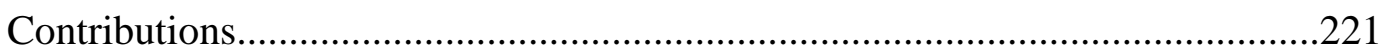

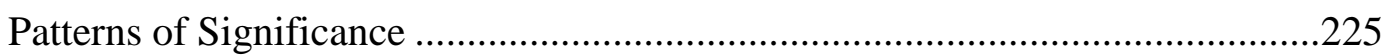

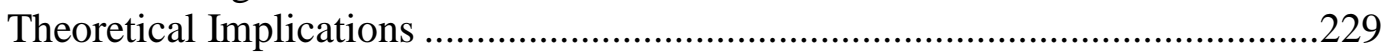

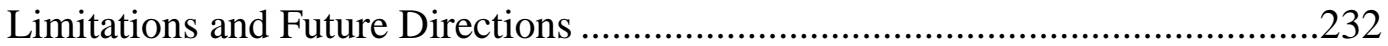

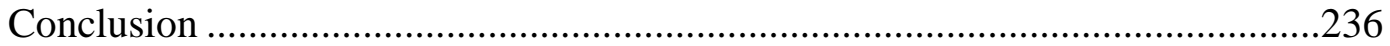

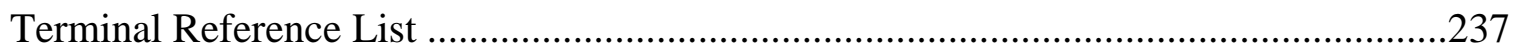

Appendix A: Pilot Study Procedure and Results ......................................................... 261

Appendix B: Lab Procedure/Lab Script..................................................................... 270 
List of Tables

\section{CHAPTER II. MANUSCRIPT I. HAVING FRIENDS AND FEELING LONELY: A DAILY-PROCESS EXAMINATION OF TRANSIENT LONELINESS, SOCIALIZATION, AND DRINKING BEHAVIOR}

Table 1.0: Bivariate correlations between study variables .68

Table 1.1: Within-person associations between loneliness, alcohol consumption, and time spent interacting with others. 62

Table 1.2: Multilevel mediation models for social and solitary outcomes.

CHAPTER III. MANUSCRIPT II. NO ONE TO TURN TO IN TIMES OF JOY: LONELINESS AND CAPITALIZATION DEFICITS

Table 2.0: Bivariate correlations among raw study variables

Table 2.1: Model fit and model comparisons for full and partial mediation .110

Table 2.2: Results for partial mediated model with transformed variables.

Table 2.3: Total and specific indirect effects and BC confidence intervals .112

Table 2.4: Probability profiles for likelihood of capitalizing on positive events .....113

\section{CHAPTER IV. MANUSCRIPT II. EXPERIMENTAL INVESTIGATION OF LONELINESS AND THE PERCEIVED PROVISION AND RECEIPT OF RESPONSIVE BEHAVIOR DURING POSITIVE-EVENT DISCLOSURE}

Table 3.0. Bivariate correlations among key study variables

Table 3.1. Group comparisons for actor induction condition

Table 3.2. Actor indirect effects of state loneliness, perceived responsiveness, state self-esteem, and perceived event importance 201

Table 3.3. Moderated mediation of chronic loneliness on induction-state lonelinesspositive affect indirect effect. .202 Table 3.4. Moderated mediation of chronic loneliness on induction-state lonelinessliking indirect effect.

Table 3.5. Moderated mediation of chronic loneliness on induction-state lonelinessenjoyment indirect effect.

Table 3.6. Moderated mediation of chronic loneliness on induction conditionperceived responsiveness- positive affect association indirect effect .205 Table 3.7. Moderated mediation of chronic loneliness on induction conditionperceived responsiveness-liking indirect effect.

Table 3.8. Moderated mediation of chronic loneliness on induction conditionperceived responsiveness-enjoyment indirect effect

Table 3.9. Group comparisons for dyad condition 208 Table 3.10. Indirect effects of perceived responsiveness on partner dyad conditioncapitalization outcomes models 209

Table 3.11. Summary of hypotheses and results. 210 
List of Figures

CHAPTER II. MANUSCRIPT I. HAVING FRIENDS AND FEELING LONELY: A DAILY-PROCESS EXAMINATION OF TRANSIENT LONELINESS, SOCIALIZATION, AND DRINKING BEHAVIOR

Figure 2.0: Perceived social support moderation for loneliness-solitary consumption associations

CHAPTER III. MANUSCRIPT II. NO ONE TO TURN TO IN TIMES OF JOY: LONELINESS AND CAPITALIZATION DEFICITS

Figure 3.0: Conceptual model for mechanisms through which loneliness relates to positive-event disclosure

Figure 3.1: Partial mediated model (final model)...........................................115

Figure 3.2: Partial mediated model with parameter estimates. 116

\section{CHAPTER IV. MANUSCRIPT III: EXPERIMENTAL INVESTIGATION OF LONELINESS AND THE PERCEIVED PROVISION AND RECEIPT OF RESPONSIVE BEHAVIOR DURING POSITIVE-EVENT DISCLOSURE}

Figure 4.0: Indirect effects of loneliness on the relationships between actor mood induction, perceived responsiveness, self-reported provision of responsive behavior, and capitalization outcome associations

Figure 4.1: Indirect effects of perceived responsiveness on the relationship between actor mood induction and capitalization outcomes

Figure 4.2: Indirect effects of state self-esteem and perceived event importance on the effects of actor induction on capitalization outcomes.

Figure 4.3: Moderation of loneliness on indirect effects of lonely mood............215

Figure 4.4: Moderation of loneliness on indirect effects of responsiveness........216 Figure 4.5: Indirect effects of perceived responsiveness on the relationship between partner dyad condition and capitalization outcomes Figure 4.6. Interaction of state loneliness and chronic loneliness on liking towards lab partner .218 Figure 4.7. Interaction of state loneliness and chronic loneliness on enjoyment of the interaction. .219

Figure 4.8. Interaction of perceived responsiveness and chronic loneliness on liking towards lab partner .220 


\section{CHAPTER 1. INTRODUCTION}

It is widely known that supportive social relationships are vital elements of human flourishing and well-being. Various theorists have argued that interpersonal relationships satisfy a fundamental human need, and that the desire to develop and maintain relationships is an essential human motivation (Baumeister \& Leary, 1995; Deci \& Ryan, 2000). One major framework with social psychology which has been used to describe the importance of social relationships is Deci and Ryan's theory of self-determination, which maintains that human beings are intrinsically motivated to fulfill the basic need of interpersonal relatedness, or feelings of closeness and connectedness with others. Reis, Sheldon, Gable, Roscoe, and Ryan (2000) demonstrate that on a daily level, such needs are best fulfilled when an individual feels unconditionally cared for and supported, which results in greater feelings of intimacy, self-esteem, vitality, and positive affect (LaGuardia \& Patrick, 2008). Overall relatedness need satisfaction is known to relate to greater positive affect and well-being, whereas low relatedness and interpersonal connectedness result in various psychological and behavioral consequences, including anxiety, depression, alienation and risk behavior (e.g., alcohol and marijuana use, early sexual activity; Deci \& Ryan, 2000; Heinrich, Brookmeyer, Shrier \& Sharah, 2006; Resnick et al., 1997).

Echoing the self-determination perspective, Baumeister and Leary (1995) propose that human beings have a pervasive drive to form and maintain lasting, positive, and significant interpersonal relationships. According to their need to belong hypothesis, the universal tendency to form social relationships is a fundamental motivation which shapes 
human emotions, cognitions, and behavior. Specifically, when the need to belong is thwarted, individuals experience affective consequences, including reduced psychological need satisfaction and positive affect, and increased levels of negative affect. Further, unfulfilled belongingness needs relate to negative physiological and psychological health outcomes (i.e., poor physical health and psychological maladjustment), and are predictive goal-direct behavior designed to remediate unsatisfied belongingness needs (Baumeister \& Leary, 1995; Sheldon \& Gunz, 2009). A prime example of this process is work by Baumeister and DeWall (2005), which demonstrates that laboratory-induced social exclusion results in significant impairments in cognitive ability, memory retrieval, and logical reasoning. Similar work has shown that priming individuals to feel lonely or ostracized predicts deficits in self-regulation (Rotenberg \& Flood, 1998) and at times more aggressive behavior (Warburton, Williams, \& Cairns, 2006).

An additional component of the need-to-belong hypothesis is that individuals are not only motivated to affiliate with individuals and groups, but are also motivated to exhibit behavior which encourages others to desire mutual affiliation and interaction (Leary, 2010). For example, early work by Mehrabian (1969) provided evidence that persons motivated to engage in close, positive social interactions are more likely to exhibit nonverbal behavior which indicates openness, friendliness, and interest (e.g., smiling, open body posture, also known as immediacy behaviors). Such behaviors are thought to promote relational value, that is the extent to which others consider a relationship with an individual as valuable and important (Leary, 2010). Perceiving that 
one's relational value is high is related to feelings of acceptance and belonging, while low perceived relational value is connected to feelings of rejection and isolation.

In extension of the self-determination and needs-based perspectives of social relationships, much work has considered the influence of social relationships and need satisfaction on psychological and physiological health and well-being. Much of the literature on social relationships, health, and well-being stems from the seminal work of sociologist Emile Durkheim (1857/1951) who proposed that a lack or breakdown of family, friend, and community ties has severe pathological outcomes, including suicide. He theorized that a lack of social ties leads to a loss of social resources, particularly those providing support and defining social roles and norms (Cohen, Brissette, Skoner, \& Doyle, 2000; Durkheim, 1951). Since the work of Durkheim, research has continuously shown that social connectedness is related to numerous health outcomes including lower mortality, healthier immune system functioning, and more health-facilitating behavior, such as exercise (Berkman \& Breslow, 1983; Cohen, 1991; House, Umberson, \& Landis, 1988). Similarly, perceiving that others are available in times of need (i.e., perceived social support) is related to greater health outcomes overall, as well as attenuated physiological and psychological responses to stress and negativity (main effect and buffering hypotheses of social support; Cohen \& Wills, 1985). Conversely, and in line with the original work of Durkheim, a perceived lack of social support and lack of connectedness with others is related to anxiety, depression, and the development of various mood disorders (Baumeister \& Leary, 1995; DeLongis, Folkman, \& Lazarus, 1988; Sarason, Sarason, \& Gurung, 2001). Additionally, failure to maintain lasting, 
positive interpersonal relationships results in a sense of deprivation, anger, and of central focus to this dissertation, loneliness (Cacioppo et al., 2000; Heinrich \& Gullone, 2006).

\section{Loneliness}

Commonly defined as the perception of social isolation (Perlman \& Peplau, 1984), or a perceived deficit in the quantity or quality of interpersonal relationships (Russell, Peplau, \& Cutrona, 1980), loneliness is a commonly-reported experience associated with feelings of dissatisfaction, unhappiness, anxiety, hostility, emptiness, boredom, and restlessness (Perlman \& Peplau, 1982; Perlman \& Peplau, 1984; Rook, 1984). Though often associated with depression, loneliness can be experienced by socially-connected and isolated individuals alike. Further, research has shown that loneliness occurs throughout the lifespan, and is not limited to socially-alienated and isolated individuals (Cacioppo, Gripp, London, Goossens, \& Cacioppo, 2015). Indeed, early loneliness theorists differentiated between forms of loneliness based on chronicity, stability, and predisposing variables or events. Specifically, Young (1982) defined chronic, situational, and transient forms of loneliness, describing chronic loneliness as long-lasting and pervasive feelings of isolation, situational loneliness as feelings of isolation and uneasiness which occur during major life transitions (e.g., moving to a new city or losing a loved one), and transient loneliness as shorter bouts of loneliness commonly experienced throughout daily life.

Much evidence exists which demonstrates that loneliness has serious mental and physical health consequences similar to those associated with objective social. Specifically, long-term experiences of loneliness are related to poorer self-reported 
physical health symptoms (Cornwell \& Waite, 2009), low immune system functioning (Glaser et al., 1985), poor cardiovascular health, and high blood pressure (Hawkley, Masi, Berry, \& Cacioppo, 2006), as well as greater mortality, disease susceptibility, cognitive decline, obesity, and poorer self-reported health symptoms (i.e., headaches, fatigue; Heinreich \& Gullone, 2009). Given the severity of these physiological health outcomes, loneliness has been of central concern within the social psychology, relationships, and health literature, with work exploring this construct spanning over the past fifty years.

Further, recent scholars and clinicians have expressed concern with growing levels of isolation and loneliness in current American society, despite increased levels of connectedness which social media and technology provide (Hawkley \& Cacioppo, 2007). Indeed, longitudinal evidence has suggested that increased internet and forms of Facebook use (i.e., non-personalized use such as scanning status updates) are actually related to less social involvement, less communication with family members, as well as increased depression and loneliness (Burke, Marlow, \& Lento, 2010; Kraut et al., 1998). These trends, along with well-established evidence for the health consequences of loneliness over time, make loneliness a timely and very relevant construct to explore within the current study of social relationships, health, and well-being.

\section{Cognitive-Discrepancy Model of Loneliness: Objective versus Subjective Isolation}

While loneliness is related to similar health outcomes as more objective levels of social isolation, it is a distinct phenomenon which is characterized by a subjective evaluation of relationship quality. One of the most basic models of loneliness is the 
cognitive discrepancy-attributional model, which proposes that the basis of loneliness is a perceived discrepancy between desired and actual interpersonal relationships (Paloutzien \& Janigian, 1987; Perlman \& Peplau, 1981). This model describes loneliness as a subjective experience based on the perception and evaluation of relationship quality, thus distinguishing loneliness from objective social isolation (Peplau, 1985). Further, the discrepancy-attributional perspective of loneliness focuses on the difference between one's desired and actual levels of social relations, rather than general levels of social contact and interaction. In line with the cognitive model, research has demonstrated that perceived and objective social isolation (loneliness and social network size) are only moderately correlated (Cornwell \& Waite, 2009; Shankur, McMunn, Banks, \& Steptoe, 2011). That is, loneliness is not synonymous with social isolation or lack of social integration, and the socially isolated should not be assumed to be among the lonely.

Though there is a clear distinction between objective and subjective reports of isolation (i.e., social isolation and loneliness), researchers have identified that the quantity of network ties, or lack thereof, is a key though distal determinant of loneliness (e.g., Cutrona, 1986; Hawkley et al., 2008). For example, previous evidence has suggested that those experiencing greater loneliness have smaller and less satisfying social networks, less frequent interactions with close friends and family, and engage in social activities less frequently than do nonlonely individuals (Jones \& Moore, 1987; Wenger, Davis, Shahtahmasebi, \& Scott, 1996). Similarly, Cutrona (1986) demonstrated that the number of kin in a given social network significantly contributes to feelings of loneliness, such that individuals with more kin exhibit lower levels of loneliness. Marital 
status and frequency of contact with kin were also significantly related to increased perceptions of support by a given network (Cutrona, 1986). In a similar vein, Bondevik and Skogstad (1998) provided evidence that low frequency of contacts with friends and neighbors is related to higher levels of loneliness. Therefore, lacking specific close relationships, frequent positive interactions within these relationships and more generally, a diverse network of social ties is thought to predispose individuals to the distressing experience of loneliness.

Though the absence of close relationships and a diverse social network may predispose individuals to feelings of loneliness, social interactions, marital relationships, and social ties are not necessarily protective factors against loneliness. As an example, recent polls show that $20-40 \%$ of married and non-married individuals in the United States report being lonely at any given time (Bekhet, \& Zauszniewski, 2012; Cacioppo \& Patrick, 2008; Wilson \& Moulton, 2010). Further, recent work has provided evidence that loneliness fluctuates within and between days as a function of social context and negative social contacts (e.g., Gross, Juvenon, \& Gable, 2002). Thus, though the presence of social ties and close relationships may relate to lower levels of chronic loneliness, it is likely and has been demonstrated that even socially connected individuals experience loneliness as a daily experience (Cacioppo \& Patrick, 2008).

Yet, despite the early theorists' distinction among different forms of loneliness (Young, 1982), as well as evidence suggesting growing rates of loneliness among socially isolated and connected individuals alike, the majority of research to date has continued to focus on chronic levels of loneliness by employing cross-sectional measures and 
methods, or has not differentiated among the forms of loneliness at all. Thus, while much is known about the correlates and consequences of chronic loneliness, less is known about transient loneliness, despite the fact that it is commonly experienced within the general population. In fact, some theorists posit that transient experiences of loneliness are less impactful and of less concern than loneliness experienced at chronic levels (Cacioppo \& Patrick, 2008). Conversely, other theorists suggest that it is imperative to examine processes through which transient loneliness may evolve into a more severe condition and find ways to prevent this transition from occurring (Perlman \& Peplau, 1981).

This dissertation takes the later view, investigating processes through which transient experiences of loneliness could develop into more chronic levels over time, as well as mechanisms which may make loneliness (at chronic and acute levels) a particularly difficult experience to overcome. To do so, this dissertation draws from an existing multidimensional framework of loneliness, which describes this affective phenomenon in terms of cognitive, affective, and behavioral manifestations and consequences (Peplau \& Perlman, 1982). Further, this framework is combined with transactional models of loneliness, which describe how the various consequences and behavioral correlates of loneliness interact with the environment to inhibit relationship processes, thus contributing to the persistence of loneliness over time. Whereas previous work using this framework does not differentiate between chronic and transient levels, this dissertation describes both forms in relation to cognitions, affect, and behavior. In doing so, this combination of studies expands on previous work by describing individual 
and relationship processes through which acute levels of loneliness can develop into more chronic levels, and thus influencing psychological and physiological health over time.

\section{Multidimensional Model of Loneliness: Affect, Behaviors, and Cognitions}

Similar to Baumeister and Leary's (1995) conceptualization of affective, motivational/cognitive, and behavioral consequences of the need to belong, theorists describe loneliness as a multidimensional construct, with affective, cognitive, and behavioral features and correlates (Peplau \& Perlman, 1979).

Loneliness as a negative-affective experience. As already described, loneliness is an unpleasant, negative-affective experience, associated with various other forms of negative affect (e.g., boredom, unhappiness, anxiety, worthlessness, and sadness; Perlman \& Peplau, 1979). Additionally, loneliness is related to greater negative affectivity and lower optimism (Davis, Hanson, Edson, \& Ziegler, 1992), greater social anxiety and lower feelings of self-worth or self-esteem (Peplau \& Perlman, 1984), as well as lower life satisfaction (e.g., Schumaker, Shea, Monfries, \& Groth-Marnat, 1993). Lonely persons also report less intimacy, trust, comfort and more negative feelings during and following social interactions (e.g., Hawkley, Burleson, Bernston, \& Cacioppo, 2003). Further, the experience of loneliness is strongly related to depression, and whereas distinct constructs, depression and loneliness typically co-occur among chronically lonely individuals (Heinreich \& Gullone, 2009).

Cognitive and perceptual deficits related to loneliness. Though loneliness is clearly manifested as a form of negative affect, it is unique in that it has strong motivational properties. Specifically, theorists assert that loneliness serves as a social 
pain or hunger which "motivates the maintenance and formation of social connections necessary for the survival of our genes" (Hawkley \& Cacioppo, 2010, p. 2). In line with this perspective, research has shown that lonely persons tend to exhibit increased social monitoring and a greater sensitivity and attentiveness to social cues, behavior which plays a role in the regulation of belongingness needs, feelings of connectedness, etc. (social-monitoring system; Gardner, Pickett, Jefferis, \& Knowles, 2010). Interestingly, though social monitoring tends to relate to greater social functioning and relationship well-being, lonely persons have a difficult time translating these social cues into appropriate behavior during interpersonal interactions (Gardner et al., 2010).

Moreover, and of greatest relevance to this dissertation, loneliness relates to more negative perceptions of social interactions, as well as negative appraisals of close relationships and social support (e.g., Heinrich \& Gullone, 2006; Solano, Batten, \& Parish, 1982; Wittenberg \& Reis, 1986). Thus, while lonely persons may be carefully in tune to the social environment and motivated to seek social relationships, they have difficulty engaging in these social interactions and have negative expectations for these interactions when they do occur. An additional cognitive correlate of loneliness relates to attributions for success and failure. Specifically, lonely persons tend to attribute failure to more stable, internal attributes (i.e., lack of skills or ability, characterological deficits), whereas they attribute successes to external and temporary causes (Anderson, Horowitz, \& French, 1983). This style of attribution is problematic, in that loneliness may influence individuals' ability to reap the benefits of positive experiences and successes. As will be described below, negative perceptions and appraisals of social interactions and external 
attributions for success have implications for positive relationship processes (i.e., capitalization; Gable, Reis, Impett, \& Asher, 2004; Langston, 1994), which not only play a large role in the development and maintenance of close relationships, but are also known to reduce momentary feelings of loneliness.

Behavioral consequences of loneliness. Behavioral correlates and consequences of loneliness have been explored in terms health and risk-related behavior, which includes the study of coping responses to loneliness and social skill deficits characteristic of lonely individuals (e.g., Jones et al., 1982; Rubenstein \& Shaver, 1982; Solano et al., 1982). In particular, research has shown that lonely persons tend to exhibit fewer healthpromoting behaviors, such as less exercise, poor nutrition, and poorer sleep quality (e.g., Cacioppo et al., 2002; Heinrich \& Gullone, 2006). Further, research examining coping responses to loneliness describes sad passivity behaviors, by which individuals attempt to alleviate feelings of loneliness through behavior such as substance use or risky sexual behavior (e.g., Heinrich \& Gullone, 2006). Interestingly, studies examining associations among loneliness and negative health behaviors (i.e., alcohol consumption) are less conclusive, showing both differential and comparable risk behaviors among lonely and less lonely individuals (e.g., Cacioppo et al., 2002; Shankur et al., 2011). Yet, much of the existing research investigating health-behavioral correlates of loneliness, such as alcohol consumption, employs cross-sectional measures of loneliness and associated health behaviors (e.g., UCLA Loneliness Scale; Russell et al., 1996), therefore limiting findings to the more stable, chronic experiences of loneliness. Further, such measures do not capture the fluctuating, state-like (i.e., transient) experiences of loneliness, which 
could be experienced by even socially connected individuals. Indeed, more work is needed to understand how transient experiences of loneliness influence health-related behavior on a daily level, which could have implications for long-term physiological health and psychological well-being.

In addition to negative health behavioral outcomes, loneliness is related to various social behavioral deficits which are thought to influence the ability to develop lasting social relationships. In particular, loneliness is associated with self and other reports of poor social skills and less responsive behavior during social interactions (e.g., Jones, Hobbs, \& Hockenbury, 1982), including lower conversational involvement and more self-focused attention (Bell, 1985). Additionally, researchers have tied loneliness to general social-withdrawal-type behavior (e.g., Cacioppo et al., 2006) and to the lower use of support-seeking behavior (Wilson \& Moulton, 2010). Further, research has shown that lonely individuals are less likely to report disclosing negative events (Wittenberg \& Reis, 1986). In addition, loneliness has been related to a lower willingness to disclose negative events, as well as difficulty managing appropriate levels of disclosure during social interactions (disclosure flexibility; Chelune, Sultan, \& Williams, 1980). That loneliness negatively influences disclosure behavior is problematic, given that this support-seeking behavior is particularly important in the development of intimacy and the maintenance of close, interpersonal relationships (Reis \& Shaver, 1988), and which could serve as an anecdote to feelings of loneliness in the moment and over time. 
CONSEQUENCES OF LONELINESS

\section{Transactional Model of Loneliness}

As clearly exhibited in Peplau and Perlman's (1979) multidimensional model, loneliness has widespread effects on cognitions, affect, and behavior. Yet, this model does not describe how these various 'symptom' of loneliness could function to maintain this negative-affective experience, or processes through which transient experiences of loneliness could develop into more chronic levels. Transactional models of loneliness provide insight into these processes, positing that the affective, cognitive, and behavioral consequences of loneliness, and in particular social behavioral deficits, interact with the environment to exacerbate and maintain this negative-affective experience (e.g., Ernst \& Cacioppo, 1998; Cacioppo, Grippo, London, Goossens, \& Cacioppo, 2015).

In particular, cognitive and behavioral deficits associated with loneliness (the current dissertation argues at both acute and chronic levels) interfere with the ability to effectively engage in positive social interactions, thus contributing to the persistence of loneliness in the moment, and growing feelings of loneliness over time. One example of this process relates to observed deficits in negative event disclosure among lonely persons. In line with transactional models of loneliness, evidence has suggested that this relationship between loneliness and disclosure behavior is explained by poor social skills and negative perceptions of social interactions (Solano, Batten, \& Parish, 1982). Additionally, early research and theory suggested that lonely persons may view selfdisclosure as risky, as it makes these individuals vulnerable to potential social rejection (Rotenberg, 1997; Wittenberg \& Reis, 1986). However, while the avoidance of selfdisclosure may be more comfortable, doing so prevents these individuals from reaping 
the social rewards of disclosure interactions (i.e., intimacy, liking, and closeness).

Ironically, lonely individuals are missing out these important social interactions which could potentially aid in the development of close relationships and reduce loneliness over time.

Loneliness and positivity deficits. Much work has considered the behaviors and experiences of lonely persons during social interactions and disclosure in general, providing a clear example of different mechanisms through which the many features of loneliness inhibit important interpersonal process, thus contributing to the persistence of loneliness over time. Yet, no work has considered how loneliness influences engagement in more positive relationship processes, such as the disclosure of positive events (e.g., Gable et al., 2004; Langston, 1994). Research within the emotions, self-regulation, and personality literature demonstrates that positive and negative processes and behavioral systems are functionally independent. That is, the absence of negative does not imply the presence of positive, and vice versa (Reis \& Gable, 2003). Recent work by Kashdan, Ferssizidis, Farmer, Adams, and McKnight (2013) considers the independence of positive and negative processes by taking a positivity-deficit perspective in exploring symptoms of social anxiety which interfere with the maintenance of close, healthy interpersonal relationships. Given the tendency for loneliness research to date to focus on negative behaviors and negative-event related processes (i.e., disclosure of negative events), future work should examine loneliness from a similar positivity-deficit perspective. In particular, research should explore how loneliness influences engagement in positive (approach-oriented) behaviors and processes, such as the disclosure of positive events, or 
capitalization, the absence of which could limit individuals from developing lasting social relationships.

Capitalization. Recent work has shown that the disclosure of positive events, or capitalization, is a particularly positive interpersonal experience which builds various personal and social resources. Additionally, perceived support during good times is arguably a better predictor of well-being and relationship outcomes, compared to perceptions of support during difficult times (Gable et al., 2004; Gable, Reis, Impett, \& Asher, 2006). Though effective capitalization interactions have been shown to reduce loneliness and increase perceptions of support, work has not considered how lonely feelings inhibit successful engagement in these positive social interactions. To the extent that capitalization has both intrapersonal and interpersonal outcomes, and is related to reduced levels of loneliness (Gable \& Reis, 2010), it would be beneficial to explore how both chronic and transient experiences of loneliness influence successful engagement in this positive relationship process. Such research would provide insight into transactional models of loneliness, which posit that loneliness and it's affective, behavioral, and cognitive correlates interfere with the development of positive, interpersonal relationships.

Interestingly, recent research has examined how social anxiety, a correlate of loneliness and common characteristic of lonely individuals, relates to behavior within lab-based capitalization interactions. Such research has shown that individuals with greater social anxiety perceive their interaction partners as less responsive during the disclosure of a positive event (i.e., capitalization attempt), exhibited less supportive 
responses to their capitalization attempt, and were thus less likely to benefit from capitalization as relationship maintenance and repair process (Kashdan et al., 2013). Additionally, interaction partners of these socially anxious individuals also reported receiving and providing a less supportive response during the capitalization interaction (i.e., positive-event disclosure). Similar research has shown individuals with low selfesteem and partners of individuals with low self-esteem reported were less likely to disclosing positive events as they anticipated the receipt of a less supportive response from their interaction partner (MacGregor, Fitzsimons, \& Holmes, 2013; Smith \& Reis, 2012).

Whereas loneliness is related to social anxiety and self-esteem, it is a distinct construct, the independent effects of which have yet to be examined in relation to behavior and cognitions within a capitalization interaction. Additionally, no research to date has examined how the loneliness of one individual influences the behaviors and perceptions of his/her interaction partner. Yet, as described by previous theories in social psychology and close relationships research, social interactions and disclosure interactions more specifically are transactional in nature. Specifically, Reis and Shaver's (1988) interpersonal model of intimacy development posits that, within disclosure interactions the behaviors and perceptions of one individual interacts with and influence the behaviors and perceptions of interaction partners. In particular, each individual's motives, needs, goals and fears influences his/her own disclosure behavior as well as response to the disclosure of his/her interaction partner. Further, perceptions of 
responsiveness are influenced by both an individual's motives, needs, goals, and fears, as well as the actual behavioral response of an interaction partner.

Clark and Lemay (2010) present a similar perspective through their integrative model of responsiveness. In particular, this model demonstrates that one individual's perceptions of responsiveness influence his/her own provision of responsive behavior, well-being, and future response seeking behavior. In turn, the provision of responsive behavior influences the perceptions, behavior, and well-being of his/her partner (Clark \& Lemay, 2010).

In line with previously established transactional models of social interaction, disclosure, and responsiveness (e.g., Clark \& Lemay, 2010; Reis \& Shaver, 1988), recent work has shown that emotional, cognitive, and behavioral consequences of loneliness function to negatively influence the experience of those interacting with lonely persons, and subsequently induce feelings of loneliness among others (induction hypothesis; Cacioppo, Fowler, \& Christakis, 2009). One example of this process relates to social interactions of lonely persons with their network members. Specifically and as described above, feelings of loneliness are related to increased shyness, hostility, anxiety, lower self-esteem, and social awkwardness. These behavioral deficits of lonely persons make interactions less enjoyable for interaction partner, who may in turn experience increased levels of loneliness and associated emotional, cognitive, and behavioral consequences. The induction hypothesis, along with previously established transactional models of selfdisclosure and responsiveness demonstrate the importance of assessing the perceptions and behaviors of both lonely persons and those with whom they interact. Such research 
would be beneficial in revealing one potential pathway through which loneliness interferes with vital relationship-building interactions and processes, thus perpetuating feelings of loneliness over time.

\section{Present Investigation}

This dissertation took a multi-study approach to address various gaps in the current loneliness literature. Using a full-cycle approach, the current study employed experimental and non-experimental methods (i.e., daily-process, cross-sectional survey, and experimental laboratory methods) to examine affective, cognitive, and behavioral correlates of chronic and acute levels of loneliness, thus contributing to a more comprehensive understanding of loneliness as consequential to intrapersonal and interpersonal behavior and well-being (Mortenson \& Cialdini, 2010). The sections that follow provide a brief introduction to the three manuscripts included in this dissertation.

\section{Chapter II Overview: Having friends and feeling lonely: A daily-process} examination of transient loneliness, socialization, and drinking behavior

The first paper (Arpin, Mohr, \& Brannan, 2015) investigates daily experiences of loneliness in relation to subsequent daily health and social behavior (i.e., social and solitary alcohol consumption, time spent interacting with others), as well as betweenperson differences in daily loneliness-behavior associations (i.e., gender, social support, relationship status). Additionally, analyses investigate the mediating role of time spent with others on loneliness-related changes in social and solitary alcohol consumption. This is the first study to date to examine loneliness-related behavior at the daily, withinpersons level of analysis. Further, results provide insight into the unique role of 
loneliness in inhibiting vital support processes within personal relationships (i.e., support seeking), as well as self-defeating patterns of behavior (i.e., solitary consumption) which could serve to increase levels of loneliness and relate to health deficits over time.

Based on known patterns of social-withdrawal responses to loneliness (e.g., Rubenstein \& Shaver, 1982), and models of negative mood-related alcohol consumption (e.g., Mohr et al., 2001), it was hypothesized that increases in loneliness would relate to increases in solitary consumption, but decreases in social consumption and time spent interacting with others. Additionally, and in line with previously established gender differences in reactivity to daily stress and negative mood (Bolger, DeLongis, Kessler, \& Schilling, 1989), it was predicted that women would exhibit stronger mood drinking slopes relative to men. Given that loneliness represents a perceived relationship deficit, or discrepancy in desired and actual social relationships (Cacioppo et al., 2006; Paloutzien \& Janigian, 1987) it was predicted the buffering effects of social support typically observed within the literature would not be observed in relation to daily increases in transient loneliness. Lastly, loneliness-related increases in solitary consumption would occur above and beyond changes in social behavior, while decreases in social consumption would be explained by decreased socialization on days with greater loneliness.

\section{Chapter III Overview: No one to turn to in times of joy: Loneliness and capitalization deficits.}

The second paper of this dissertation investigated associations among chronic loneliness, perceptions of support, self-esteem, perceptions of event-importance, and the 
likelihood of disclosing positive events in a large-scale student survey of college student health and interpersonal relationships. While previous research has explored chronic loneliness in relation to various negative behavioral processes and social deficits (i.e., negative health behavior, poor social skills, low self-esteem, maladaptive attributions of positive event and successes, negative perceptions of social relationships), no research to date has examined how loneliness influences engagement in positive relationship processes, such as capitalization. Capitalization, or positive-event disclosure, is a key relationship maintenance and repair process related to positive intrapersonal (e.g., positive affect) and inter-personal outcomes (e.g., relationship satisfaction, intimacy; Gable et al., 2004; Gable et al., 2006). Whereas previous research has examined capitalization as relating to increased perceptions of support and reduced loneliness (Gable \& Reis, 2010), this study examined these variables from a novel perspective by exploring how chronic levels of loneliness inhibit the process of capitalization from unfolding.

Based on previous evidence of negative-event disclosure deficits related to loneliness (Wittenberg \& Reis, 1986), it was predicted that loneliness would relate to a lower likelihood of capitalizing on positive events by disclosing them to others. Additionally, this association would be explained by lonely individual's lower perceptions of social support, self-esteem, and less positive perceptions of event importance. Findings are interpreted in light of the current loneliness and close relationships research, as well as Kashdan et al.'s (2013) positivity-deficit perspective. In particular, processes are discussed through which lonely individuals are experiencing 
deficits in positive interpersonal processes, which may contribute to the persistence of loneliness over time.

\section{Chapter IV Overview: Experimental investigation of loneliness and the perceived provision and receipt of responsive behavior during positive event-disclosure}

The third paper examined the influence of transient loneliness on individual and interaction partner experiences within a lab-based, positive event-sharing interaction. Expanding on the previous loneliness literature, this study took an ecologically valid approach by examining self- and other- reports of perceptions and behavior within the interaction. Overall, the purpose of this study was to explore the extent to which momentary feelings of loneliness influence positive social behavior and cognitions (i.e., provision and perceived receipt of responsive behavior), and impair the intrapersonal and interpersonal benefits of capitalization (i.e., positive affect, partner liking).

Specifically, this study investigated how state feelings of loneliness versus feelings of social connectedness (experimental induction) influence individual perceptions of responsiveness and the provision of responsive behavior, as well as levels of positive affect, enjoyment of and interaction, and partner liking following the eventsharing interaction. In addition, analyses explored whether 'nonlonely' partners (socialconnectedness induction) of 'lonely' participants (loneliness mood induction) reported similar deficits in the receipt and provision of responsive behavior, as well as less positive affect, liking towards the other participant, and enjoyment of the interaction following the capitalization interaction task. Further, the extent to which perceived responsiveness explained deficits in positive affect, enjoyment of the interaction, liking 
towards lab partner were examined for 'lonely' individuals and their interaction partners. Importantly, these effects were compared to those of individuals in interaction dyads wherein both participants received a connectedness induction. Lastly, the study examined the extent to which individuals at chronic levels of loneliness, as assessed in an initial assessment, experienced worse outcomes of the capitalization interaction (i.e., positive affect, liking towards lab partner, enjoyment of the interaction), compared to individuals at lower levels of loneliness.

Transactional models of loneliness posit that the behavioral consequences of loneliness, such as poor social skills, deficits in self-disclosure, and low conversational involvement in social interactions, operate on the environment to exacerbate and maintain loneliness (Ernst \& Cacioppo, 1998). Additionally, the induction hypothesis posits that loneliness and its behavioral consequences contribute to the spread of loneliness to those with whom lonely individuals interact (Cacioppo, Fowler, \& Christakis, 2009). Further, as argued by Reis and Shaver (1988) and Clark and Lemay (2010), social interactions and self-disclosure more specifically are interpersonal, transactional processes within which each individual's cognitions, motives, and past experiences influence the likelihood of self-disclosure as well as responsiveness to partner disclosure. Given these theoretical models of loneliness, self-disclosure, and social interaction, it was hypothesized that individuals induced to feel lonely (versus socially connected) and their interaction partners would report receiving and providing a less supportive response in the context of an event-sharing interaction, and report lower levels of positive affect, less partner liking, and less enjoyment of the interaction following a positive event-disclosure interaction. 
This study was the first of its kind to examine the effects of transient or state loneliness on social behavior and perceptions through a randomized experimental mood induction, which allowed for the examination of the effects of acute levels of loneliness above and beyond its affective correlates (i.e., general negative affect) and controlling for individual differences in chronic levels of loneliness. Thus, this study makes a substantial contribution to the existing loneliness literature, which primarily focuses on behavioral consequences of chronic loneliness in the context of negative behavioral processes (e.g., alcohol consumption, lack of negative-event disclosure). In contrast to this approach, this study considered positivity deficits related to transient experiences of loneliness within the context of a positive social interaction, which may be consequential to the development and maintenance of close relationships.

\section{Summary}

In sum, this dissertation presents three complete manuscripts which investigate the effects of acute and chronic levels of loneliness on intrapersonal and interpersonal health and social behavior, through various affective, cognitive, and behavioral processes. The first study investigated within-person associations among transient loneliness and context-specific consumption, unpacking individual differences and the indirect effects of time spent with others. The second paper examined the extent to which loneliness relates to the disclosure of positive events, and as various mechanisms which influence engagement in this positive relationship process (i.e., self-esteem, social support, and perceived event importance). Finally, the third manuscript explored causal associations among acute experiences of loneliness and individual and interaction partner perceptions 
and behavior during an event-sharing interaction. Whereas existing loneliness research has relied heavily on cross-sectional and survey methods, this dissertation examined the consequences of loneliness at the between and within-person levels of analysis, through experimental and non-experimental methods (i.e., cross-sectional surveys and dailyprocess methods). Further, this combination of studies unpacks intrapersonal (i.e., moodrelated alcohol consumption) and interpersonal (self-disclosure, and perceived responsiveness) processes, expanding the current understanding of loneliness as consequential to individual health and well-being, to include interpersonal functioning and the development and maintenance of close relationships. 
CHAPTER II. MANUSCRIPT I. HAVING FRIENDS AND FEELING LONELY: A DAILY-PROCESS EXAMINATION OF TRANSIENT LONELINESS, SOCIALIZATION, AND DRINKING BEHAVIOR

\begin{abstract}
Loneliness is a well-known indicator of relationship deficits, with potentially severe consequences on health and well-being (Perlman \& Peplau, 1981). Research has employed cross-sectional methods to examine behavioral consequences of loneliness (e.g., Cacioppo et al., 2002). However, within-person associations between daily fluctuations in loneliness and subsequent behavioral outcomes have yet to be explored. Using a sample of community-dwelling adults $(\mathrm{N}=49)$, the authors examined associations between daily loneliness on daily time with others, and subsequent contextspecific alcohol consumption (i.e., social and solitary consumption), and individual differences in these patterns of behavior. Daytime loneliness significantly and uniquely predicted patterns of social behavior and context-specific consumption. Time with others mediated loneliness-social consumption associations, but not loneliness-solitary consumption relationships. These findings contribute to existing literature by demonstrating the unique properties of solitary versus social consumption as behavioral responses to loneliness, thus addressing inconsistent findings regarding the effects of loneliness on alcohol consumption.
\end{abstract}

Keywords: Loneliness, social relationships, daily-process methodology, alcohol consumption 
Theorists argue that interpersonal relationships satisfy a fundamental human need, and that the desire to develop and maintain relationships motivates much of human behavior. One major social psychological framework which has been used to describe the importance of social relationships is Deci and Ryan's (2000) theory of self-determination, which maintains that human beings are intrinsically motivated to fulfill the basic need of interpersonal relatedness, or feelings of closeness and connectedness with others. Much work has demonstrated that being well-integrated in a diverse network of social ties predicts greater physical health and engagement in health-facilitating behavior (e.g., Berkman \& Breslow, 1983). Conversely, a lack of strong social ties and perceived deficits in social connectedness are related to negative outcomes including depression, engagement in risk-related behavior, and loneliness.

Loneliness, or perceived social isolation, is one common indicator of relationship deficits associated with feelings of dissatisfaction, anxiety, emptiness, and boredom (Perlman \& Peplau, 1981). Though commonly associated with objective levels of social isolation, loneliness is a distinct psychological phenomenon. Specifically, and as proposed by the cognitive discrepancy model, loneliness is based on a perceived discrepancy between desired and actual interpersonal relationships (Dykstra \& Fokkema, 2007; Peplau \& Perlman, 1979). Accordingly, research has demonstrated that perceived and objective social isolation (i.e., social network size) are only moderately correlated (e.g., Cornwell \& Waite, 2009). That is, loneliness is not synonymous with social isolation or lack of social integration, and the socially isolated should not be assumed to be among the lonely. 
Despite the distinction between loneliness and objective levels of social connectedness, loneliness has been related to similar mental and physical health consequences as objective levels of social isolation. Specifically, long-term experiences of loneliness are related to poorer self-reported physical health, low immune system functioning, greater mortality, disease susceptibility, cognitive decline, and obesity (e.g., Heinreich \& Gullone, 2009). Given the severity of these physiological health outcomes, loneliness has been of central concern within the social psychology, close relationships, and health literature, with work exploring this construct spanning over the past fifty years. Moreover, recent research reveals that rates of loneliness are on the rise, with 30$40 \%$ of Americans reporting feelings of loneliness at any given time (e.g., Wilson \& Moulton, 2010). Thus, the effect of loneliness on health and well-being is a particularly timely process to explore.

\section{Multidimensional and Transactional Models of Loneliness}

A key framework describing the various consequences of loneliness is Peplau and Perlman's (1979) multidimensional model of loneliness. This model draws from existing research to describe loneliness as a multi-dimensional construct, with affective, cognitive, and behavioral manifestations, many of which can be used to explain the long-term health effects of this negative-affective experience. In example, research has shown that loneliness is a distressing affective experience, associated with greater negative affectivity, lower life satisfaction, and low self-esteem (e.g., Davis, Hanson, Edson, \& Ziegler, 1992). Further, lonely persons generally report more negative perceptions of social interactions, increased social monitoring and sensitivity to social threats (cognitive 
correlates of loneliness; Gardner, Pickett, Jefferis, \& Knowles, 2010). Of greatest relevance to the current study are behavioral outcomes and correlates of loneliness (e.g., Perlman \& Peplau, 1981). Specifically, research has provided evidence that lonely persons exhibit fewer health-promoting behaviors (e.g., poor nutrition, poorer sleep quality; Cacioppo et al., 2002; Heinrich \& Gullone, 2006), and greater health risk-related behaviors, such as alcohol consumption and substance use (e.g., Cacioppo et al., 2002; Shankur, McMunn, Banks, \& Steptoe, 2011). Interestingly, research has revealed both differential and comparable risk behaviors among lonely and less lonely individuals.

However, much of the research to date examining loneliness and health behavior has employed cross-sectional methods and assesses general levels of consumption. These methods do not capture the fluctuating, everyday experiences of loneliness which may uniquely relate to behavioral outcomes. Interestingly, early theorists differentiated chronic loneliness (long-lasting feelings of isolation) from more transient experiences, which are known to fluctuate across days as a function of social context and are more commonly experienced throughout daily life (Gross et al., 2002; Young, 1982). Yet, despite these distinct typologies, the majority of current research focuses on chronic levels of loneliness as well as associated health and behavioral outcomes. Thus, very little is known about behavioral responses to and consequences of transient loneliness, which may be experienced by socially-connected and isolated individuals alike.

Loneliness researchers have elucidated a diversity of behavioral strategies which people use in response to chronic levels of loneliness (e.g., Rubenstein \& Shaver, 1982). Among these responses are solitary behaviors or active solitude (e.g., listening to music, 
journaling) through which individuals reflect on the experience of loneliness, seeking social contact, and sad passivity behaviors through which individuals attempt to alleviate feelings of loneliness through substance use (e.g., Heinrich \& Gullone, 2006). Researchers have also tied loneliness to general social-withdrawal-type behavior (e.g., Cacioppo et al., 2008) and the use of fewer active coping strategies, such as supportseeking (Wilson \& Moulton, 2010). Ironically, though loneliness signals the need to reconnect, it is associated with increased sensitivity to social threat, fear of rejection, and thus avoidance of social participation and activity (e.g., Rokach \& Neto, 2000). Thus, while seeking social contact may help to alleviate feelings of loneliness, many individuals are likely to withdraw from social behavior in times of loneliness out of fear for potential rejection.

Transactional models of loneliness posit that these various behavioral consequences of loneliness interact with the environment to maintain and even exacerbate loneliness over time (Ernst \& Cacioppo, 1998). For example, by withdrawing from social interaction because of negative perceptions, lonely persons' behavior perpetuates feelings of loneliness. Additionally, certain health behavioral responses to loneliness, such as substance use (i.e., sad passivity), may actually serve to exacerbate levels of lonely mood and thus relate to poorer health outcomes over time. Specifically, and as demonstrated in the alcohol myopia literature (e.g., Steele \& Joseph, 1990), particular forms of negative mood-related use (i.e., solitary consumption) actually exacerbate negative moods and stressors, because attention is focused on precipitating stressors rather than distractions present in more social settings (Armeli et al., 2003). 
Thus, sustained patterns of loneliness-related consumption may contribute to the maintenance of this negative-affective experience. In the current study, we examine one aspect of the transactional processes, specifically patterns of daily loneliness-related behavior (i.e., alcohol consumption and social-withdrawal) which, when repeated over time, could contribute to the maintenance of loneliness, and thus negative health outcomes.

\section{Daily Mood-Related Alcohol Consumption and Drinking Context}

Given our focus on daily patterns of behavior coinciding with the transactional model, and the relative dearth of research examining behavioral outcomes of daily fluctuations in loneliness, our work is primarily focused on state or transient levels of loneliness. Accordingly, the present study uses daily-process methodology to explore how transient experiences of loneliness relate to subsequent reports of social behavior (i.e., time spent with others) and context-specific alcohol consumption (i.e., social and solitary consumption), behavioral outcomes which have been previously examined as general responses to loneliness. Daily-process research, which employs multiple assessments per day per participant, is particularly beneficial for loneliness research, as it allows for the examination of fluctuating experiences of loneliness, subsequent behavioral outcomes, and individual differences in these within-person patterns of behavior. Such methodology minimizes retrospective bias, affording the examination of antecedents, correlates, and consequences of daily experiences, as well as betweenperson differences in daily patterns of behavior (Bolger, Davis, \& Rafaeli, 2003). 
Previous daily-process research has revealed valuable information about withinperson patterns of fluctuating moods and behavioral outcomes, demonstrating that the regulation of negative affect is a primary motive for alcohol consumption (Cooper, 1994). Further, research examining mood-related alcohol consumption has also revealed that the context of drinking matters, affirming that social and solitary alcohol consumption are psychologically distinct drinking behaviors with different antecedents and consequences (Cooper, Frone, Russell, \& Mudar, 1995). In particular, daily negative interpersonal experiences are associated with increased solitary consumption (i.e., drinking-to-cope), while positive interpersonal experiences are predictive of greater drinking in social contexts, or enhancement drinking (Mohr et al., 2001).

Whereas previous daily-process research has provided evidence for negative affect-motivated drinking behavior, research has not examined fluctuations in loneliness as an immediate precursor to daily alcohol consumption. Further, previous research exploring loneliness and health behavior does not distinguish between social and solitary drinking experiences. Yet, research and theory describe responses to loneliness as uniquely relating to behaviors within social and solitary contexts. Specifically, sad passivity responses likely include increases in solitary drinking behavior, whereas socialwithdrawal responses relate to decreased social behavior (Rubenstein \& Shaver, 1982). To the extent that transient loneliness is a negative-affective experience which has been related to general levels of alcohol consumption, it would be important to examine within-person relationships among loneliness and context-specific drinking behavior. 
Distinguishing between social and solitary consumption as outcomes of daily loneliness would provide insight into behavioral processes through which loneliness relates to negative health outcomes over time (i.e., multidimensional and transactional models of loneliness). Specifically, previous research has elucidated the differential effects of social versus solitary consumption on short-term and long-term health. For instance, social consumption tends to have the greatest stress-dampening effects compared to solitary consumption (e.g., Armeli et al., 2003). Further, negative moodrelated drinking could lead to the development of alcohol problems, particularly as it reflects drinking-to-cope motivation (Cooper et al., 1995). Additionally, distinguishing between drinking contexts would also address inconsistencies in the current loneliness literature, which typically averages over drinking context, particularly in terms of the relation of loneliness to alcohol use and abuse. Thus, in the current study, we examine how loneliness relates to changes in social versus solitary consumption. To demonstrate the importance of distinguishing between social and solitary consumption, we assess how daily fluctuations in loneliness relate to total daily consumption, irrespective of drinking context.

Further, though chronic loneliness has also been related to social-withdrawal, research has not considered whether daily fluctuations in loneliness relate to subsequent decreases in social behavior. Thus, in the current study, we also examine how daily increases in loneliness relate to subsequent decreases in time spent interacting with others. To differentiate these changes in socialization from social and solitary 
consumption, we examine whether increases in daily loneliness relate to changes in social and solitary consumption indirectly through decreases in social behavior.

\section{Differences in Mood-Drinking Relationships: Stress-Vulnerability Models}

In addition to negative mood-related and context-specific drinking behavior, daily-process research has revealed important differences in the extent to which individuals exhibit affect-motivated patterns of drinking behavior (i.e., stressvulnerability model; Hussong, Hicks, Levy, \& Curran, 2001). In particular, work examining the stress-vulnerability model has demonstrated individual differences in negative mood-related drinking associations, particularly in terms of gender, drinking motivations, and social support (e.g., Hussong et al., 2001).

Evidence from the alcohol literature provides mixed support in terms of gender differences in mood-drinking relationships. For example, some researchers have shown that men exhibit stronger associations between daily negative moods and alcohol consumption (Armeli et al., 2000), while others have demonstrated unique patterns of mood-related consumption among women, relative to men (i.e., Hussong et al, 2001). More general research within the stress and coping literature provides evidence of gender differences in reactivity to daily stress and moods, showing the effects of daily interpersonal conflict on mood are stronger for women relative to men (e.g., Mohr et al., 2003). These findings are consistent with theoretical arguments describing the strong interpersonal and communal orientations of women, which may make experiences of loneliness more salient (Wood \& Eagly, 2002). Researchers have similarly explored individual differences in responses to loneliness, providing evidence that women tend to 
exhibit greater frequency of alcohol intoxication at high levels of loneliness relative to men (Bonin, McCreary, \& Sadava, 2000). However, no research to date has examined whether women and men differ in drinking responses to daily increases in loneliness. To the extent that loneliness is a stressful, negative emotional experience, it would be important to consider the role of gender when examining patterns of daily lonelinessrelated drinking behavior.

In addition, research has explored the role of social support as a buffer to negative mood, stress, and interpersonal conflict. Under traditional stress-buffering models, social support would reduce the effects of loneliness on behavioral outcomes, such as alcohol consumption (e.g., Cohen \& Wills, 1985). Indeed, previous research has shown that positive associations among daily stressors and subsequent alcohol use are weaker for individuals at high levels of support (e.g., Wang, Liu, Zhan, \& Shi, 2010). However, it is possible that this negative-affective experience interacts uniquely with perceptions of support. For individuals at high levels of support, loneliness may act as a signal that typical support resources are unavailable. Further, increases in loneliness may be more salient for those with generally high levels of support compared to those with low support, given the contrast to what these individuals generally experience within their relationships. Thus, while perceived social support typically predicts better stress-related outcomes, it is possible that this process is inhibited for transient experiences of loneliness, which signal a discrepancy between what is desired in relationships and what is received in the moment. 
In sum, though previous research and theory has considered loneliness and its and behavioral consequences, there is a relative dearth of research unpacking transient experiences of loneliness and associated behavioral outcomes. Further, existing research examining the effects of loneliness on alcohol consumption tends to average over drinking context, despite known responses to loneliness which differentially relate to social and solitary behavior. Lastly, examining gender and support as moderators of daily loneliness-behavior associations would provide insight into cognitive-discrepancy models of loneliness, which describe loneliness as based in a perceptual deficit between desired and actual social relationships.

\section{Current Investigation}

The present study utilizes daily-process methodology to explore temporal associations among increases in loneliness and subsequent health and social behavioral outcomes (i.e., social consumption, solitary consumption, time spent interacting with others), consistent with known categories of responses to loneliness. In addition, we explore the moderating effect of gender and social support on within-person lonelinessbehavior associations, as well as the mediating effects of time spent with others on loneliness-drinking associations. Specifically, the following research questions were explored: (1) Do changes in daytime loneliness relate to subsequent drinking behavior in solitary or social contexts?; (2) Do changes in daytime loneliness relate to time spent with others at a later time point?; and (3) Do these within-person associations vary by gender and social support? As an additional exploratory step, we considered whether changes in social and solitary consumption were explained by changes in social behavior. 
Specifically, we examined the extent to which time spent with others mediated loneliness-solitary and loneliness-social consumption associations. Additionally we considered individual differences in these indirect effects based on gender and social support.

Based on previous daily-process research and known responses to loneliness (e.g., sad passivity and social-withdrawal), we predicted that individuals would exhibit greater solitary consumption and less social consumption subsequent to that day's increases in loneliness. Further, we expected that on days with greater loneliness participants would exhibit decreases general social behavior (i.e., time spent interacting with others). We also predicted that women would exhibit stronger increases in consumption following increases in loneliness. Additionally, drawing from discrepancy-based models of loneliness, we anticipated that individuals at higher levels of social support would exhibit stronger reactivity to daily increases in loneliness. Lastly, based on previous dailyprocess research, wherein negative moods and social interactions predicted drinking at home alone above and beyond the effects of time spent interacting with others (Mohr et al., 2005), we anticipated that changes in social behavior help to explain the effects of loneliness on lower levels of social consumption at a later time point. However, we did not anticipate the reduction in time spent with others would explain subsequent increases in loneliness-related solitary consumption. 


\section{Method}

\section{Participants and Procedure}

Forty-nine community-dwelling adults (50\% female; average age $=36, S D=$ 16.16) participated as a part of a larger daily-process study investigating interpersonal relationships, moods, and health behaviors. Ninety percent of participants were Caucasian, $2 \%$ were African-American, and 6\% were Hispanic/Latino/Spanish, and 4\% identified as other/multiracial. Thirty-six percent of participants were married or cohabitating. Seventy-six percent of participants were employed, earning average income ranging from $\$ 27,000$ to $\$ 44,000$, with a mean education level of a bachelor's degree or lower. Participants were recruited through internet postings, flyers and local newspapers dispersed through the greater Portland Metropolitan area.

Given our interest in studying moderate-to-heavy alcohol consumers, we did not include light consumers in our general study sample (i.e., men who drank less than 3 drinks per day/12 per week, women who drank less than 2 drinks per day/6 per week), nor did we include individuals who met the criteria for alcohol dependence or current abuse (as assessed by the Computerized Diagnostic Interview Schedule IV; Robins, Cottler, Bukholz, \& Compton, 1995), or those reporting illicit substance use. Those showing signs of clinical depression were also excluded from the study.

Eligible participants were invited to complete informed consent and an initial survey (i.e., gender, age, perceived support), followed by a 30-day, daily assessment of moods, social interactions, and health behaviors completed via a hand-held electronic interviewer (i.e., ELI). Daily surveys took about five minutes to complete and were 
completed at three interval time points throughout each day: 10:00-11:30 a.m. (Interval 1), 4:00-5:30 p.m. (Interval 2) and 8:30-10:00 p.m. (Interval 3). The ELI sounded an audible alert at random points during each window offered a vibration function, allowing participants to delay responding up to 3 hours. Participants could earn up to $\$ 185$ and inclusion in a cash lottery for participation, depending on completeness of surveys.

Of the 49 participants who consented to the study, technical problems with the ELI rendered data for 2 participants unusable. The remaining participants provided a potential 4230 person-day observations ( $47 \times 90$ surveys). Of those, $2 \%$ were outside the 3-hour grace period, in which case participants responded to questions regarding that day's experiences. Responses which were not appropriate to address lagged mooddrinking relationships and thus not included. In preliminary analysis, we conducted hypothesized models including data reported outside the 2-hour grace-period, within a 1and 2-hour grace-period, or only observations occurring within the survey windows described above. Significance and interpretation of results were comparable using original and extended timeframes. For the present analysis, we retained observations occurring within a 2-hour grace period to maintain power of observations, while minimizing overlap of interval time-points. Thirty-five additional observations were eliminated as they were between the 2- and 3-hour time windows. Based on interviews completed within a 2-hour grace period, a total of 3329 surveys were completed from a possible 4111 , resulting in $81 \%$ compliance, which is comparable to previous daily diary studies (e.g., Feldman, Downey, \& Shaffer-Neitz, 1999). 


\section{Initial Assessment Measures}

Basic demographics. Participants reported on basic demographics, including gender, age, income, and personality.

Perceived social support. General perceptions of support were assessed via the Interpersonal Support Evaluation List (ISEL; Cohen, Mermelstein, Karmarck, \& Hoberman, 1985). Participants responded to a series of statements probing perceptions of support availability on a 4-point scale (1-Definitely false; 4-Definitely true). Example items included "If I wanted to have lunch with someone, I could easily find someone to join me" and "There is someone I can turn to for advice about handing problems with my family." Items demonstrated acceptable internal consistency, consistent with previous uses of this measure (Cronbach's alpha $=.87$ ).

\section{Daily Measures}

Loneliness. At each daily interval participants completed a mood assessment adapted from the Positive and Negative Affect Schedule (PANAS; Watson, Clark, \& Tellegen, 1988). Daily loneliness was assessed as one of the negative affect items on the PANAS. Participants were asked to indicate how lonely they felt in the moment, based on a 5-point Likert-type scale (0- Not at all, 4-Extremely). Previous researchers have validated the use of single-item measures of loneliness against the well-validated UCLA Loneliness Scale (e.g., Pressman et al., 2005). Further, research has shown that moods can be reliability assessed with single items (e.g., Burisch, 1984; Myers \& Diener, 1995), with the advantage of face validity and brevity, thus reducing participant fatigue (Larsen \& Fredrickson, 1999). Daily reports of loneliness were aggregated across the thirty-day 
study as a proxy for mean-levels of loneliness, which we included as a level-2 covariate in all of our analyses.

Daily alcohol consumption. Participants recorded alcohol consumption at each interval, indicating the number of alcohol beverages consumed since the previous interview (i.e., Interval 1, drinking which occurred the previous evening; Interval 2, drinking which occurred during the day; Interval 3, drinking since the previous interview). Participants were trained to recognize standard drink sizes, based on NIAAA (National Institute on Alcohol Abuse and Alcoholism) criteria (i.e., one drink defined as one $12 \mathrm{oz}$. can/bottle beer, $8 \mathrm{oz}$. malt liqueur, $5 \mathrm{oz}$. glass of wine, $12 \mathrm{oz}$. wine cooler, or 1.5 oz. of straight liquor/mixed drink). At each time point, participants indicated whether drinking had occurred at home or away from home, and whether they were "alone," "interacting with drinking others" "interacting with non-drinking others," "not interacting and not drinking," or "not interacting and others drinking," and the number of drinks consumed in each context $(0$ to $>12)$.

To reduce the number of context-specific categories, analyses were limited to drinking which occurred home alone (i.e., SOLITARY) and away from home while interacting with others also drinking (i.e., SOCIAL). Drinks consumed in other contexts were too few to evaluate the appropriateness of combining them with drinks home alone or away with others. Previous research has utilized these specific categories as indicators of social and solitary drinking contexts (e.g., Mohr et al., 2013). Total consumption was computed by summing the total number of drinks consumed at home and away from home, irrespective of specific drinking contexts. Lastly, previous research has shown that 
self-reports of alcohol use within 24-hours of consumption are highly correlated with objective measures of use, thus supporting the reliability of this recall method (Perrine, Mundt, Searles, \& Lester, 1995).

Interacting with others. At each measurement time-point, participants noted the number of hours $(0$ to $>8)$ spent interacting with others since the previous interview (i.e., INTERACTING). Previous research has reliably assessed daily time spent interacting with others in vivo, via similar checklists of daily events (i.e., daily events checklist; Armeli et al., 2003). Evening time interacting was recorded in the following day's Interval-1 survey assessment.

\section{Data Analysis}

To examine within-person associations between daytime loneliness and subsequent behavior, we conducted multilevel regressions using Hierarchical Linear Modeling software (HLM, v.6.0; Raudenbush, Bryk, Cheong, \& Congdon, 2000), which accounts for the unbalanced, nested nature of daily diary data. A two-level model was specified, consisting of Level 1 within-person and Level-2 between-person effects. Level1 predictors were person-centered and Level-2 predictors were grand mean-centered (Hofmann \& Gavin, 1998). Person-centered loneliness was lagged by participant ID, allowing for the prediction of consumption from loneliness at an earlier time point. All analyses controlled for gender, age, mean-levels of loneliness, income, and neuroticism at level-2, variables which previous research has shown to influence rates of alcohol consumption and responses to loneliness. 
Preliminary analyses revealed low rates of consumption during the daytime interval (SOLITARY, $M=.02, S D=.19$; SOCIAL, $M=.09, S D=.55$ ), which is consistent with previous diary studies of moderate drinkers for whom the majority of drinking occurs in the evening (e.g., Armeli et al., 2003; Swendsen et al., 2000). Given the high number of structural zeros for Interval 2 rates of consumption, analysis for the drinking outcomes were limited to Interval 1 and Interval 3 assessments. Additionally, it should be noted that response options for drinking were count variables and thus nonnormally distributed. Further, data for both drinking contexts were zero-inflated. Therefore, we modeled SOCIAL, SOLITARY outcomes on a Poisson sampling distribution (Coxe, West, \& Aiken, 2009; Raudenbush \& Bryk, 2002).

To test within-person associations among daily loneliness and subsequent behavior, we modeled within-person outcomes (i.e., SOCIAL, SOLITARY, and INTERACTING) as a function of within-person predictors (i.e., loneliness, Level-1). Further, we examined mean-level differences in Level-1 outcomes, by modeling Level-1 intercepts as a function of Level-2 predictors/covariates. To control for day-of-the-week and time-of-day effects, six dummy coded day-of-the-week variables (Tuesday contrast), and one time-of-day dummy code (i.e., evening) were included as fixed effects, as per suggestion of Bryk and Raudenbush (1992) ${ }^{1}$. Equation 1 provides an example of Level-1 and Level-2 models for the SOCIAL drinking outcome. Identical Level-1 and Level-2 models were run for SOLITARY and INTERACTING outcomes.

$$
\begin{gathered}
\text { Social Consumption }_{\mathrm{it}}=b_{0 \mathrm{i}}+b_{1 \mathrm{i}}\left(\text { Loneliness }_{\mathrm{it}-1}\right)+b_{2 \mathrm{it}}(\text { Mon })+b_{3 \mathrm{it}}(\text { Wed })+ \\
b_{4 \mathrm{it}}(\text { Thurs })+b_{5 \mathrm{it}}(\text { Fri })+b_{6 \mathrm{it}}(\text { Sat })+b_{7 \mathrm{it}}(\text { Sun })+b_{8 \mathrm{it}}(\text { Evening })+\mathrm{e}_{\mathrm{it}}
\end{gathered}
$$


DAILY LONELINESS AND DRINKING BEHAVIOR

$$
\begin{aligned}
& b_{0 \mathrm{i}}=\gamma_{00}+\gamma_{01}(\text { Age })+\gamma_{02}(\text { Mean 30-Day Loneliness })+\gamma_{03}(\text { Gender })+ \\
& \gamma_{04}(\text { Income })+\gamma_{05}(\text { Neuroticism })+\mathrm{u}_{0 \mathrm{i}} \\
& b_{1 \mathrm{i}}=\gamma_{10}+\mathrm{u}_{1 \mathrm{i}}
\end{aligned}
$$

where person $i$ 's SOLITARY at time point $t$ is modeled as a function of person $i$ 's intercept (i.e., expected SOLITARY at his/her average level of loneliness), loneliness at the previous time point $t-1$, and a residual $e_{i t}$. The Level-1 slope for loneliness $\left(b_{1 \mathrm{i}}\right)$ can be interpreted as the estimated change in social consumption associated with an increase in person $i$ 's loneliness from his/her mean levels.

To examine inter-individual differences in within-person associations, Level-1 loneliness slopes were modeled as a function of gender and social support. Equation 4 shows the intercepts model with control variables, and Equation 5 shows the Level-2 regression model including cross-level interactions of gender and social support on Level-1 loneliness-SOCIAL slopes.

$$
\begin{aligned}
& b_{0 \mathrm{i}}=\gamma_{00}+\gamma_{01}(\text { Age })+\gamma_{02}(\text { Mean 30-Day Loneliness })+\gamma_{03}(\text { Gender })+ \\
& \gamma_{04}(\text { Income })+\gamma_{05}(\text { Neuroticism })+\gamma_{06}(\text { Social Support })+\mathrm{u}_{0 \mathrm{i}} \\
& b_{1 \mathrm{i}}=\gamma_{10}+\gamma_{11}(\text { Gender })+\gamma_{12}(\text { SocSupport })+\mathrm{u}_{1 \mathrm{i}}
\end{aligned}
$$

As an exploratory step, we also examined the indirect effects of time spent with others on loneliness-SOCIAL and loneliness-SOLITARY associations, to further differentiate loneliness-related consumption from reductions in social behavior. To do so, we utilized methods originally developed by Kenny, Korchmaros, and Bolger (2003) for the estimation of a series of random effects lower-level mediation models. Using specification methods developed by Bauer, Preacher, and Gil (2006), we estimated 
confidence intervals for indirect effect point estimates. As this particular method required the utilization of normal approximation, and to our knowledge no methods have been developed for testing lower-level random effects mediation for non-normal outcomes, we log-transformed the drinking outcomes. Lastly, we explored the moderating effects of gender and social support on significant mediation models.

\section{Results}

\section{Descriptive Statistics}

Across the 30-day study, participants consumed an average of 2.09 total drinks per drinking day $(S D=1.86)$, with an average of 6.97 evenings drinking home alone and 6.25 evenings drinking with others. Participants consumed a maximum of 6 solitary drinks per drinking day, and 13 social drinks per drinking day. Average solitary consumption across the 30-day study was consumption was $1.71(S D=1.31)$ drinks per solitary drinking day, and mean social consumption was $2.97(S D=2.56)$ drinks per social drinking day. Across the 30-day study, which included drinking and nondrinking days, participants reported an average of .18 solitary drinks and .38 social drinks. Additionally, participants consumed $20 \%$ in social contexts, and $17 \%$ in solitary contexts out of total drinks consumed. Within-person correlations among social and solitary consumption were significant and negative $(b=-.04, p=.002)$, such that greater solitary consumption related to less social consumption on a given day.

Average daily loneliness was $1.42(S D=.50)$, and participants interacted with others an average of 2.85 hours per days on which they interacted with others. Across the 30-day study (on interacting and non-interacting days), participants reported an average 
of 1.50 hours interacting with others. Within-person variability in loneliness (average within-person standard deviation; $S D=.52$ ) was comparable to levels of between-person variance. There were no gender differences in mean-levels of social or solitary consumption, time spent with others, or loneliness over the 30-day study. Younger participants reported greater mean-levels of social consumption $(b=-.04, p<.001)$. Individuals earning a lower income $(b=-.43, p<.001)$, and individuals with greater loneliness across the 30-day study $(b=1.09, p=.03$ ) reported greater mean-levels of solitary consumption. Descriptive statistics and bivariate correlations are displayed in Table 1.0.

\section{Multilevel Loneliness Associations}

We first examined whether increases in daytime loneliness were related to increases in SOLITARY at a later time point (Table 1.1). As expected, loneliness significantly related to subsequent SOLITARY within-person $(b=.44, p<.001$; exponentiated slope estimate $=1.55,95 \%$ CI $[1.31,1.75])$, such that individuals reported greater SOLITARY on days with greater loneliness. Thus, it can be said that a one unit increase in loneliness was related to an increase in SOLITARY drinks at a later time point by $55 \%$, or by about one drink. Despite the absence of significant slope variance, $u_{1 i}=.43, \chi^{2}(39)=51.17, p=.09$, it was appropriate to test for moderation given substantive theoretical justification (e.g., Snijders \& Bosker, 1999), as previously articulated for gender and social support. Gender significantly moderated the association between loneliness and SOLITARY $(b=.47, p=.001$; exponentiated slope estimate $=$ $1.60,95 \%$ CI $[1.22,2.11])$, with women reporting significant increases in SOLITARY 
following days with greater loneliness $(\gamma=.91, p<.001)$, while loneliness-SOLITARY associations were not significant for men $(\gamma=-.14, p=.18)$. There was also a marginally significant moderating effect of perceived social support on loneliness-SOLITARY associations $(b=.02, p=.05$; exponentiated slope estimate $=1.02,95 \% \mathrm{CI}[1.00,1.05])$. Interestingly, individuals at higher levels of support exhibited stronger increases in solitary consumption on days with greater loneliness $(\gamma=.57, p<.001)$ relative to those who reported lower levels of support $(\gamma=.28, p<.001)$.

Next, we examined whether increases in daytime loneliness were related to decreases in subsequent SOCIAL (Table 1.1). As expected, on days with greater loneliness, participants reported significant decreases in subsequent SOCIAL $(b=-.24, p$ $=.02$; exponentiated slope estimate $=.76,95 \%$ CI $[.67, .96])$. Thus, it can be said that a one unit increase in loneliness was related to a decrease in SOCIAL by $25 \%$, or by about three fourths of a drink. Significant variance in within-person loneliness-SOCIAL slopes warranted the examination of inter-individual differences, $u_{1 i}=.28, \chi^{2}(41)=93.07, p<$ .001. However, the moderating effects of gender and social support on lonelinessSOCIAL slopes were not significant.

To ensure that relationships observed among loneliness and levels of consumption were representative of unique patterns of drinking behavior, we examined the extent to which daytime loneliness related to subsequent total daily consumption, irrespective of drinking context. Daytime loneliness did not significantly relate to subsequent total levels of consumption $(b=.07, p=.75$; exponentiated slope estimate $=1.07,95 \% \mathrm{CI}[.87,1.1])$. 
Despite significant slope variance, $u_{1 i}=.07, \chi^{2}(38)=94.98, p<.001$, there were no individual differences in this association, based on gender or social support.

We then examined within-person associations among loneliness and subsequent INTERACTING, finding that daytime increases in loneliness related to subsequent decreases in INTERACTING $(b=-.17, p=.03$; exponentiated slope estimate $=.84,95 \%$ CI [.81, .99]). Thus, following increases in loneliness, individuals spent less time with others. Significant variance in within-person loneliness-INTERACTING associations, $u_{1 i}$ $=.05, \chi^{2}(39)=60.42, p=.02$, warranted the exploration of individual differences in loneliness-interacting slopes. However, the moderating effects of gender $(b=.03, p=$ $.83)$ and social support $(b=-.00, p=.75)$ on loneliness-INTERACTING associations were not significant (Table 1.1).

\section{Multilevel Mediational Analyses}

As an exploratory step, and to differentiate decreases in time spent with others from decreases in social consumption and increases in solitary changes in social and solitary consumption, we investigated the mediating effects of time spent with others on loneliness-drinking behavior associations. Specifically, we tested a series of random effects, lower-level mediational models specifying INTERACTING as the mediator for loneliness-SOLITARY and loneliness-SOCIAL associations (Kenny et al., 2003).

We first estimated the indirect effects of INTERACTING on loneliness-SOCIAL associations, controlling for gender, age, mean 30-day loneliness, income and neuroticism, as well as mean 30-day time spent interacting with others. Further, we specified random effects for all three causal pathways of the mediational model (i.e., $a_{j}$, 
$\left.b_{j}, c^{\prime}{ }_{j}\right)$. As reported in Table 1.2 , two of the three causal pathways significantly varied across persons (i.e., loneliness-INTERACTING $\left[a_{j}\right]$ and interacting-SOCIAL $\left[b_{j}\right]$ ), and both of these pathways were also significantly different from zero. Specifically, loneliness significantly related to decreased time spent with others $(b=-.03, p=.01)$, and time spent with others was related to greater social consumption $(b=.05, p<.001)$; upon the inclusion of time spent with others in the model, the original direct effect of loneliness on subsequent SOCIAL was no longer significant $(b=.002, p=.63)$. Further exploration revealed a significant indirect effect of INTERACTING on lonelinessSOCIAL associations (indirect effect $=-.001, p=.048 ; 95 \%$ CI [-2.38E-03, -8.746E-06]), such that loneliness-related reductions in subsequent social consumption were due to decreases in general social behavior. Given significant variability in paths $a_{j}$ and $b_{j}$ and significant indirect effects, we conducted an exploratory moderated mediation, examining inter-individual differences in indirect effects based on gender and levels of social support. However, results did not provide support for moderation.

Next, we examined the indirect effect of INTERACTING on lonelinessSOLITARY associations, again specifying random effects for all three causal pathways in the model (i.e., $a_{j}, b_{j}, c^{\prime}{ }_{j}$ ). All three causal pathways varied across persons, and on average these paths were significantly different than zero (Table 1.2). Specifically, loneliness significantly predicted increases in solitary consumption $(b=.01, p=.02)$ and reductions in time spent with others $(b=-.03, p<.001)$. Accordingly, time spent with others related to decreases in solitary consumption $(b=-.01, p=.02)$. Yet, there were no significant indirect effects of time spent with others on the loneliness-SOLITARY 
association. That is, loneliness related strongly to increases in subsequent solitary consumption over and above decreases in time spent with others.

\section{Discussion}

In the present study, we sought to expand existing research on health behavioral correlates and responses to chronic loneliness by examining transient loneliness on a daily level, unpacking behavioral processes related to this negative-affective phenomenon. Using daily-process methodology and lagged multilevel models, we specified time-contingent associations between loneliness and social and drinking behaviors consistent with known responses to loneliness. In doing so, we revealed that increases in loneliness were related to increases in solitary consumption and decreases in general time spent with others at a later time point. Additionally, increases in loneliness were related to decreases in social consumption. We also provided evidence for individual differences in loneliness-solitary consumption associations, countering known models of support-buffering by showing that individuals with greater support exhibited stronger loneliness-related increases in solitary consumption. Additionally, we differentiated among changes in general social behavior and social and solitary consumption by exploring the indirect effects of time spent with others on lonelinessdrinking associations. In doing so, these results provide insight into problematic patterns of drinking behavior related to daily experiences of loneliness.

Average within-person patterns of loneliness-related behavior revealed in this study are in line with known responses to chronic levels of loneliness. Specifically, results are consistent with sad passivity and social-withdrawal responses to loneliness, 
through which individuals attempt to attenuate their loneliness through alcohol use, and by avoiding social interactions (Rubenstein \& Shaver, 1982). Results are also consistent with previous daily-process research examining negative mood-related, context - specific alcohol consumption, whereby negative mood-related consumption frequently occurs in solitary but not social contexts (e.g., Mohr et al., 2001). The results of the current study provide additional evidence of sad passivity responses to loneliness, as well as support for solitary drinking contexts as characteristic of negative mood-related drinking behavior.

We also demonstrated the importance of differentiating between social and solitary consumption, as well as the unique effects of loneliness on solitary consumption, by examining a series of multilevel mediational models, wherein general social behavior mediated loneliness-drinking associations. Results from our mediational models provide evidence for two patterns of loneliness-related behavior. Specifically, results demonstrate sad passivity behaviors in the form of solitary consumption, and social-withdrawal which resulted in decreased social consumption. Our findings provide preliminary evidence of problematic patterns of drinking behavior related to daily experiences of loneliness, which could relate to health deficits and increased levels of loneliness over time.

That loneliness continued to predict increases in solitary consumption, above and beyond reductions in general social behavior, while the effects of loneliness on social consumption were explained by reductions in social behavior, is indicative of maladaptive patterns of behavior associated with transient experiences of loneliness. Although alcohol consumption is known to reduce stress in the short-term (i.e., stress- 
dampening; Armeli et al., 2003), evidence within the stress-dampening literature suggests that negative mood-related solitary consumption actually increases stress and negative mood, relative to social consumption which has greater stress-dampening effects. Consistent with this, research examining the restrictive effects of consumption on cognitive functioning (i.e., alcohol myopia; Steele \& Joseph, 1990), has demonstrated that solitary consumption in particular exacerbates the effect of stress on mood. It is the pattern of increased solitary drinking behavior following increases in loneliness which is potentially harmful, and which can lead to alcohol abuse, even at lower levels of consumption (Creswell, Chung, Clark, \& Martin, 2013). Additionally, this pattern of behavior likely inhibits individuals from pursuing more effective responses (i.e., pursuing meaningful social interactions), thus exacerbating loneliness over time. Though these results have the potential to make a large contribution to the existing literature, future research should attempt to replicate these patterns of loneliness-related drinking and social behavior.

The current study also provides insight into inconsistent findings within the literature regarding the effects of loneliness on alcohol use (e.g., Cacioppo et al., 2002; Shankur et al., 2011). However, these studies average over drinking context rather than examining social and solitary drinking behaviors distinctly. As demonstrated by our results, averaging over drinking contexts likely cancels out the effects of loneliness on consumption, given the opposite directions of loneliness-social and loneliness-solitary coefficients (see Table 1.1). Our findings affirm theoretical models arguing for the 
distinctiveness of different drinking behaviors, which have distinct antecedents (e.g., Cooper, 1994).

An additional contribution of the current study relates to our consideration of individual differences in daily loneliness-behavior associations. In particular, we examined the role of gender and social support in influencing loneliness-related drinking behavior and time spent with others. Our results revealed that women were more negatively influenced by the transient experience of loneliness. These findings are consistent with recent loneliness research demonstrating that women are more likely to report greater frequency of intoxication than men at high levels of chronic loneliness (Bonin, et al., 2000). Additionally, these findings are consistent with previous research examining gender differences in patterns of negative mood-related consumption, specifically work by Hussong et al. (2001), whereby women demonstrated stronger associations among daily sad mood and alcohol consumption, while men demonstrated stronger sad mood-drinking relationships in other time frames. Drawing from theory describing the strong interpersonal orientation characteristic of women, we anticipated that women would exhibit stronger loneliness-related increases in alcohol consumption. Our findings support this prediction. Further, our findings provide evidence in support of cognitive-discrepancy models of loneliness, whereby the experience of loneliness occurs upon a perceived discrepancy of what is desired or expected and what is received in the moment. This experience may be more salient among those oriented towards interpersonal relationships. 
We also demonstrated individual differences in the effects of loneliness on solitary consumption based on perceived social support. As posited by the discrepancy hypothesis (Dykstra \& Fokkema, 2007), loneliness is not synonymous with objective levels of social isolation, but rather it occurs when what is experienced deviates from what one desires or expects within a relationship. Given the perceptual basis of this negative-affective experience, even the most socially-connected individuals can experience loneliness (Peplau \& Perlman, 1979). We interpret this result in view of perceived responsiveness, whereby perceiving that relationships are unavailable or unresponsive is a powerful experience with detrimental effects on well-being and relationship functioning, even among those with typically high levels of support (Clark \& Lemay, 2010). Given that transient loneliness is indicative of a discrepancy between what is expected in relationships and what is experienced in the moment, it is possible that transient loneliness is particularly salient for those who generally experience high levels of support, as it contrasts typical feelings of support availability. Therefore, social support may not provide the same buffering effects for alcohol consumption as seen with general negative affect and daily stress.

Additionally, it may be that experiences of daily loneliness inhibit individuals from fully engaging in and benefitting from socially-supportive interactions with those in their support networks. Indeed, previous research has shown that lonely individuals tend to perceive their social interactions as more negative, and that even acute experiences of loneliness elicit feelings of social anxiety, low-self-esteem, and poor social skills, social deficits which influence the provision and receipt of social support (e.g., Peplau \& 
Perlman, 1979). Thus, on days with greater loneliness, even those with typically high levels of social support may be limited in their perceptions and interactions with others. Future research should explore the extent to which transient experiences of loneliness relate to similar social-perceptual and behavioral deficits at the daily level, thus inhibiting support-seeking behavior.

However, it is important to note that this study employed a moderate-to-heavy drinking sample. As such, light drinkers, depressed individuals, and illicit substance users were not included in our study sample. Thus our findings may not be generalizable to light drinkers, or those suffering from depression and illicit substance users. Light consumers, who were not included in this study due to the sporadic nature of drinking, may exhibit different patterns of behavior following increases in loneliness. In particular, support-seeking behavior and increased time interacting with others may be more normative among light or non-drinking samples, wherein we might expect to see positive relationships between loneliness and increased social interaction for these individuals. Alternatively, these individuals may exhibit non-drinking-related, sad passivity responses to loneliness (e.g., crying, over-eating) which similarly lend to maladaptive patterns of behavior over time. Future research should continue to explore daily associations among loneliness and subsequent behavioral responses, both in drinking and non-drinking contexts, and among a more diverse sample of participants (i.e., abstainers and drinkers).

Although we revealed individual differences in loneliness-solitary consumption associations, there were no moderating effects of gender or social support on loneliness- 
social consumption or loneliness-interacting with others associations, nor were there individual differences in the indirect effects of time spent interacting on lonelinessSOCIAL associations. Yet, given significant variance in our loneliness-social consumption and loneliness-interacting slopes, it is clear that some individuals were decreasing their social behavior (i.e., social consumption and interacting with others) to a greater extent than others, and that some individuals may have actually increased these behaviors on days with greater loneliness. Thus, research should continue to explore other factors (e.g., personality, attachment) which influence withdrawal versus supportseeking responses to loneliness.

\section{Limitations}

Despite the study's strengths, there are limitations to note, particularly the use of a single-item measure of loneliness. Whereas multiple items are preferred when assessing constructs, studies have shown that moods can be reliably measured with single items (e.g., Myers \& Diener, 1995). Furthermore, studies have validated the use of single-item measures of loneliness against the traditional well-validated UCLA Loneliness Scale (e.g., Pressman et al., 2005). As we measured loneliness and subsequent behavior thrice per day over thirty days, a single item measure of loneliness afforded the advantage of brevity and reduction of response fatigue (Larsen \& Fredrickson, 1999).

Additionally, our study did not include a validated measure of trait loneliness to compare with daily levels of loneliness. Although inclusion of a trait-level measure would be ideal, we aggregated daily loneliness across three daily time-points and computed the average of this value across the thirty-day study for each participant. This 
allowed us to control for mean-levels of loneliness in our level-2 model, serving as a proxy for trait loneliness. Further, in controlling for mean levels of loneliness, we were able to estimate how fluctuations in loneliness influenced consumption, above and beyond levels of loneliness participants typically experienced. Lastly, while the present study examines patterns of behavior consistent with known responses to loneliness, we did not directly measure self-reported responses to loneliness (e.g., sad passivity, socialwithdrawal, etc.). However, in assessing temporal sequences of behavior following increases in loneliness, we were able to observe patterns of behavior outside of individuals' awareness, thus providing for a more objective assessment of the behavioral consequences to loneliness less susceptible to retrospective and recall bias (Reis \& Gable, 2000). In modeling loneliness at the within-person level of analysis, this study makes a meaningful contribution to the existing literature.

It is also important to note that this sample was comprised of individuals with generally low levels of loneliness, thus limiting generalizability to higher levels of loneliness. However, individuals at higher levels of loneliness would likely show less variation in daily loneliness and consumption, thus making it difficult to observe significant within-person, loneliness-drinking slopes. Yet, person-centering allowed for the examination of fluctuations around each individual's baseline level of loneliness. Thus, we examined how deviations from one's average levels of loneliness, related to subsequent behavioral responses. Another consideration is the role of third variables as explanations for the results of the current study. Of note are personality differences and demographic variables (i.e., neuroticism, income, gender, age) known to play a role in 
general patterns of alcohol use, and mood and stress-related consumption (e.g., Keyes \& Hasin, 2008). To control for the potential variable effects, we included various level-2 covariates in all of our analyses (i.e., age, gender, neuroticism, income, etc.), thus ruling out these potential third variables as explanations for our findings.

Lastly, though our sample size is consistent with those utilized in previous dailyprocess research ( $\mathrm{N}=47$; e.g., Liu, Wang, Zhan, \& Shi, 2009), there is the possibility our analyses were limited in statistical power. Specifically, as delineated by Maas and Hox (2005), slope estimates for Level-2 effects are potentially prone to bias when the number of high-order observations is below $\mathrm{N}=50$. Thus, our sample size may have limited our ability to detect between-person differences (i.e., cross-level interaction, multilevel moderated mediation). Thus, the moderating effects of gender and social support on loneliness-solitary consumption associations, as observed in this study, should be interpreted with caution. However, our primary hypotheses of interest were focused on within-person patterns of behavior. Based on criteria provided by Kreft and DeLeeuw (1998), who argue that 30 observations per 30 participants allows for sufficient power to examine multilevel relationships (total of 900 person-day observations), our sample size was sufficiently powered to examine relationships of interest $(\mathrm{N}=329$ person-day observations). To provide evidence of the robustness of these within-person effects, we include estimates of confidence intervals for all of our parameter estimates, as well as for the random effects, lower-level mediation point estimate. 


\section{Conclusion}

Using daily-process methodology, this investigation examined temporal associations among loneliness and subsequent behavioral outcomes, revealing patterns of behavior consistent with known responses to loneliness (i.e., social-withdrawal, alcohol consumption). Further, this study provides evidence suggestive of individual differences in daily responses to loneliness, demonstrating that transient loneliness is a particularly powerful experience for women and individuals with higher levels of social support. In revealing variability in loneliness-drinking relationships, this study leaves room for future research to investigate individual difference variables which may explain who else drinks or withdraws in response to daily loneliness. Lastly, this study revealed unique effects of loneliness on daily solitary consumption, irrespective of decreases in social interaction, providing evidence of a potentially problematic behavior pattern which could contribute to the development negative health outcomes (e.g., alcohol problems) and the persistence of loneliness over time. 


\section{References}

Armeli, S., Mohr, C., Tennen, H., Todd, M., Carney, M. A., Affleck, G., \& Hromi, A. (2003). A daily-process examination of the stress-response dampening effects of alcohol consumption. Psychology of Addictive Behaviors, 17(4), 266-276.

Bauer, D. J., Preacher, K. J., \& Gil, K. M. (2006). Conceptualizing and testing random indirect effects and moderated mediation in multilevel models: new procedures and recommendations. Psychological Methods, 11(2), 142.

Berkman, L. D., \& Breslow, L. (1983). Health and ways of living. New York, New York: Oxford University Press.

Bolger, N., Davis, A., \& Rafaeli, E. (2003). Diary methods: Capturing life as it is lived. Annual Review of Psychology 54, 579-616.

Bolger, N., DeLongis, A., Kessler, R.C., \& Schilling, E. A. (1989). Effects of daily stress on negative mood. Journal of Personality and Social Psychology, 57(5), 808-818.

Bonin, M. F., McCreary, D. R., \& Sadava, S. W. (2000). Problem drinking behavior in two community-based samples of adults: Influence of gender, coping, loneliness, and depression. Journal of Addictive Behaviors, 14(2), 151-161.

Bryk, A. S., \& Raudenbush, S. W. (1992). Hierarchical linear models: Applications and data analysis methods. Newbury Park: Sage.

Burisch, M. (1984). Approaches to personality inventory construction: A comparison of merits. American Psychologist, 39, 214-227.

Cacioppo, J. T., Hawkley, L. C., Crawford, L. E., Ernst, J. M., Burleson, M. H., Kowalewski, R. B., Malarkey, W. B., Van Cauter, E. \& Bernston, G. G. (2002). 
DAILY LONELINESS AND DRINKING BEHAVIOR

Loneliness and Health: Potential mechanisms. Psychometric Medicine, 64(407417).

Cacioppo J. T., \& Patrick B. (2008). Loneliness: Human nature and the need for social connection. New York: W. W. Norton \& Company.

Clark, M. S., \& Lemay, E. P. (2010). Close relationships. Handbook of Social Psychology.

Cohen, S., Mermelstein, R., Kamarck, T., \& Hoberman, H. M. (1985). Measuring the functional components of social support. In Social support: Theory, research and applications (pp. 73-94). Springer Netherlands.

Cohen, S., \& Wills, T. A. (1985). Stress, social support, and the buffering hypothesis, Psychological Bulletin, 98(2), 310.

Cooper, M. L. (1994). Motivations for alcohol use among adolescents: Development and validation of a four-factor model. Psychological Assessment, 6(2), 117-128.

Cooper, M. L., Frone, M. R., Russell, M., \& Mudar, P. (1995). Drinking to regulate positive and negative emotions: a motivational model of alcohol use. Journal of Personality and Social Psychology, 69(5), 990.

Coxe, S., West, S. G., \& Aiken, L. S. (2009). The analysis of count data: A gentle introduction to Poisson regression and its alternatives. Journal of Personality Assessment, 91(2), 121-136.

Cornwell, E. Y., \& Waite, L. J. (2009). Social disconnectedness, perceived isolation, and health among older adults. Journal of Health and Social Behavior, 50(1), 31-48. 
DAILY LONELINESS AND DRINKING BEHAVIOR

Creswell, K. G., Chung, T., Clark, D. B., \& Martin, C. S. (2013). Solitary Alcohol Use in Teens Is Associated With Drinking in Response to Negative Affect and Predicts Alcohol Problems in Young Adulthood. Clinical Psychological Science.

Cronbach, L. J. (1951). Coefficient alpha and the internal structure of tests. Psychometrika, 16(3), 297-334.

Davis, S. F., Hanson, H., Edson, R., \& Ziegler, C. (1992). The relationship between optimism-pessimism, loneliness, and level of self-esteem in college students. College Student Journal, 26(2), 244-247.

Deci, E. L., \& Ryan, R. M. (2000). The "what" and "why" of goal pursuits: Human needs and the self determination of behavior. Psychological Inquiry, 11(4), 227-268.

Dykstra, P.A. Fokkema, T. (2007). Social and emotional loneliness among divorced and married men and women: Comparing the deficit and cognitive perspectives. Journal of Applied Social Psychology, 29, 1-12.

Ernst, J. M., \& Cacioppo, J. T. (1998). Lonely hearts: Psychological perspectives on loneliness. Applied and Preventive Psychology, 8. 1-22.

Feldman, S., Downey, G., \& Shaffer-Neitz, R., 1999. Pain, negative mood, and perceived support in chronic pain patients: A daily diary study of people with relax sympathic dystrophy syndrome. Journal of Consulting and Clinical Psychology, 67.

Gable, S. L., Gonzaga, G. C., \& Strachman, A. (2006). Will you be there for me when things go right? Supportive responses to positive event disclosures. Journal of Personality and Social Psychology, 91(5), 904. 
Gerstein, L. H., \& Tesser, A. (1987). Antecedents and responses associated with loneliness. Journal of Social and Personal Relationships, 4(3), 329-363.

Gross, E. F., Juvenon, J., \& Gable, S. L. (2002). Internet use and well-being in adolescence. Journal of Social Issues, 58, 75-90.

Heinriech, L. M., \& Gullone, E. (2006). The clinical significance of loneliness: A literature review. Clinical Psychology Review, 26(6), 695-718.

Hofmann, D. \& Gavin, M. (1998). Centering decisions in hierarchical linear models: Implications for research in organizations. Journal of Management, 24(5), 623641.

Hussong, A. M., Hicks, R. E., Levy, S. A., \& Curran, P. J. (2001). Specifying the relations between affect and heavy alcohol use among young adults. Journal of Abnormal Psychology, 110(3), 449.

Kenny, D. A., Korchmaros, J. D., \& Bolger, N. (2003). Lower level mediation in multilevel models. Psychological Methods, 8(2), 115.

Keyes, K. M., \& Hasin, D. S. (2008). Socio-economic status and problem alcohol use: the positive relationship between income and the DSM-IV alcohol abuse diagnosis. Addiction, 103(7), 1120-1130.

Kreft, I. G., \& De Leeuw, J. (1998). Introducing Multilevel Modeling. Sage.

Larsen, R. J., \& Fredrickson, B. L. (1999). Measurement issues in emotion research. In D. Kahneman, E. Diener, \& N. Schwarz (Eds.), Well-being: The foundations of hedonic psychology (pp.40-60). New York: Russell Sage Foundation. 
Liu, S., Wang, M., Zhan, Y., \& Shi, J. (2009). Daily work stress and alcohol use: testing the cross-level moderation effects of neuroticism and job involvement. Personnel Psychology, 62(3), 575-597.

Maas, C. J., \& Hox, J. J. (2005). Sufficient sample sizes for multilevel modeling. Methodology: European Journal of Research Methods for the Behavioral and Social Sciences, 1(3), 86.

Mohr, C. D., Armeli, S., Ohannessian, C. M., Tennen, H., Carney, A., \& Affleck, G. (2003). Daily interpersonal experiences and distress: Are women more vulnerable? Journal of Social and Clinical Psychology, 22(4), 393-423.

Mohr, C. D., Armeli, S., Tennen, H., Carney, M.A., Affleck, G., \& Hromi, A. (2001). Daily interpersonal experiences, context, and alcohol consumption: Crying in your beer and toasting to good times. Journal of Personality and Social Psychology, 80(3), 489-500.

Mohr, C. D., Armeli, S., Tennen H., Temple, M., Todd, M., Clark, J., \& Carney, M. A. (2005). Moving beyond the keg party: A daily-process study of college student drinking motivations. Psychology of Addictive Behaviors, 19(4), 392-403.

Mohr, C. D., Brannan, D., Wendt, S., Jacobs, L., Wright, R., \& Wang, M. (2013). Daily mood-drinking slopes as predictors: A new take on drinking motives and related outcomes. Psychology of Addictive Behaviors, 27(4), 944.

Myers, D. G., \& Diener, E. (1995). Who is happy? Psychological Science, 6(1), 10-19. Peplau, L. A., \& Perlman, D. (1979). Blueprint for a social psychological theory of loneliness. Love and Attraction, 101-110. 
Perlman, D., \& Peplau, L. A. (1981). Toward a social psychology of loneliness. Personal Relationships, 3, 31-56.

Perrine, M. W., Mundt, J. C., Searles, J. S. \& Lester, L. S. (1995). Validation of daily self-report alcohol consumption using interactive voice response (IVR) technology. Journal of Studies on Alcohol, 56, 487-490.

Pressman, S. D., Cohen, S., Miller, G. E., Barkin, A., Rabin, B. S., \& Treanor, J. J. (2005). Loneliness, social network size, and immune response to influenza vaccination in college freshmen. Health Psychology, 24(3), 297.

Raudenbush, S. W., \& Bryk, A. S. (2002). Hierarchical linear models: Applications and data analysis methods. Thousand Oaks, CA: Sage Publications, Inc.

Reis, H. T., \& Gable, S. L. (2000). Event-sampling and other methods for studying everyday experience. In H. T. Reis \& C. Judd (Eds.), Handbook of Research Methods in Social and Personality Psychology, (pp. 190-222). New York: Cambridge University Press.

Reis, H. T., Sheldon, K. M., Gable, S. L., Roscoe, J., Ryan, R. M. (2000). Daily wellbeing: The role of autonomy, competence, and relatedness. Personality and Social Psychology Bulletin, 26(4), 419-435.

Robins, L. N., Cottler, L., Bucholz, K., \& Compton, W. (1995). The Diagnostic Interview Schedule for DSM-IV. Washington University School of Medicine, St. Louis, MO. 
Rokach, A., \& Neto, F. (2000). Coping with loneliness in adolescence: A cross-cultural study. Social Behavior and Personality: An International Journal, 28(4), 329341.

Rubenstein, C. M., \& Shaver, P. (1982). The experience of loneliness. In L. A. Peplau \& D. Perlman (Eds.), Loneliness: A sourcebook of current theory, research, and therapy (pp. 206-223). New York: Wiley-Interscience.

Russell, D. W. (1996). UCLA Loneliness Scale (Version 3): Reliability, validity, and factor structure. Journal of Personality Assessment, 66(1), 20-40.

Shankur, A., McMunn, A., Banks, J., \& Steptoe, A. (2011). Loneliness, social isolation, and behavioral and biological health indicators in older adults. Health Psychology, 30(4), 377-385.

Steele, C. M., \& Joseph, R. A. (1990). Alcohol myopia: Its prized and dangerous effects. American Psychologist, 921-933.

Swendsen, J. D., Tennen, H., Carney, M. A., Affleck, G., Willard, A., \& Hromi, A. (2000). Mood and alcohol consumption: An experience sampling test of the selfmedication hypothesis. Journal of Abnormal Psychology, 109, 198-204.

Wang, M., Liu, S., Zhan, Y., \& Shi, J. (2010). Daily work-family conflict and alcohol use: Testing the cross-level moderation effects of peer drinking norms and social support. Journal of Applied Psychology, 95(2), 377.

Watson, D., Clark, L. A., \& Tellegen, A. (1988). Development and validation of brief measures of positive and negative affect: The PANAS scales. Journal of Personality and Social Psychology, 54(6), 1063-1070. 
Wilson, C., \& Moulton, B. (2010). Loneliness among older adults: A national survey of adults 45+. Prepared by Knowledge Networks and Insight Policy Research. Washington, DC: AARP. Nonartists, (45).

Wood, W., \& Eagly, A. H. (2002). A cross-cultural analysis of the behavior of women and men: Implications for the origins of sex differences. Psychological Bulletin, 128(5), 699-727.

Young, J. E. (1982). Loneliness, depression, and cognitive therapy: Theory and application. In L. A. Peplau \& D. Perlman (Eds.), Loneliness: A sourcebook of current theory, research, and therapy. New Work, NY: Wiley Inter-science. 


\section{Footnotes}

${ }^{1}$ In supplementary analyses, we controlled for weekend versus weekday via a dummy code. Our results were nearly identical to those estimated in our original model. Thus, it is clear that our findings generalize across both weekends and weekdays. To retain consistency with previous research examining daily mood-related alcohol consumption, we present results from our original model included six day-of-the-week dummy codes. 


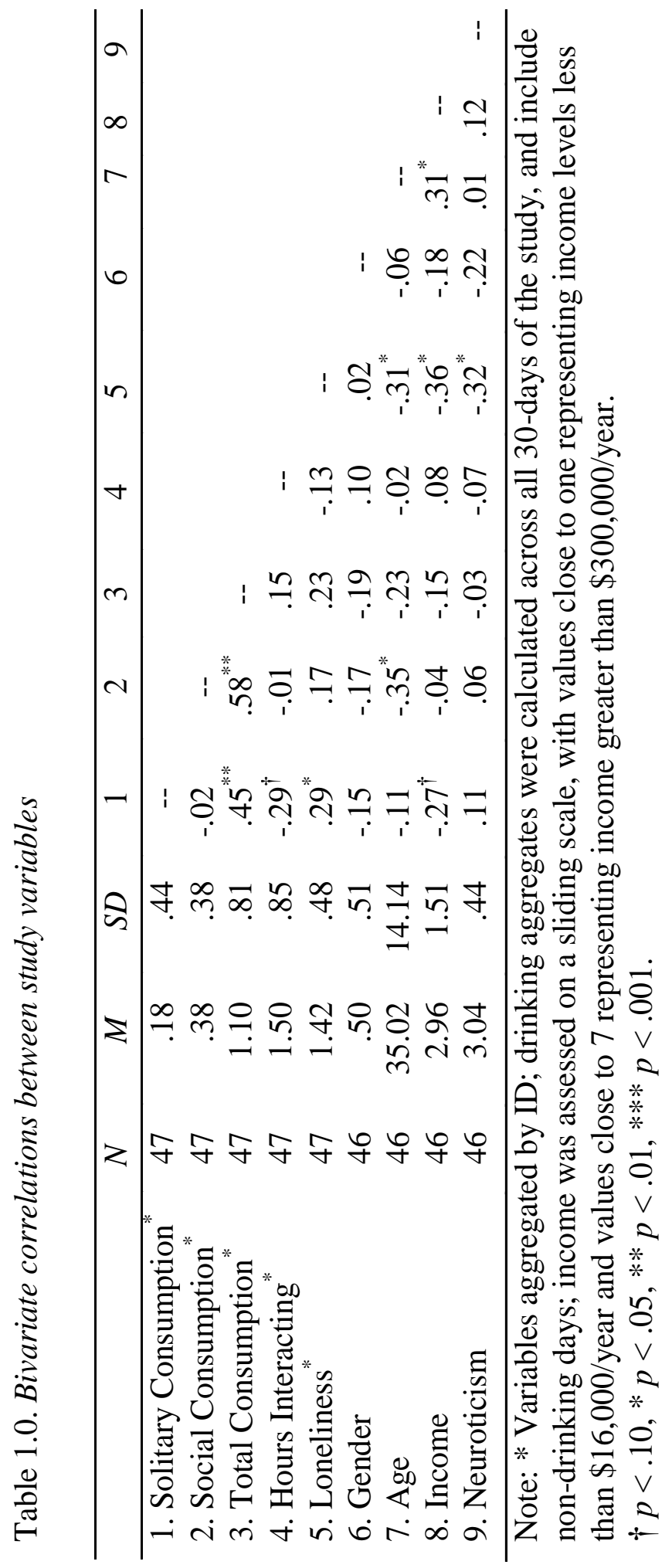




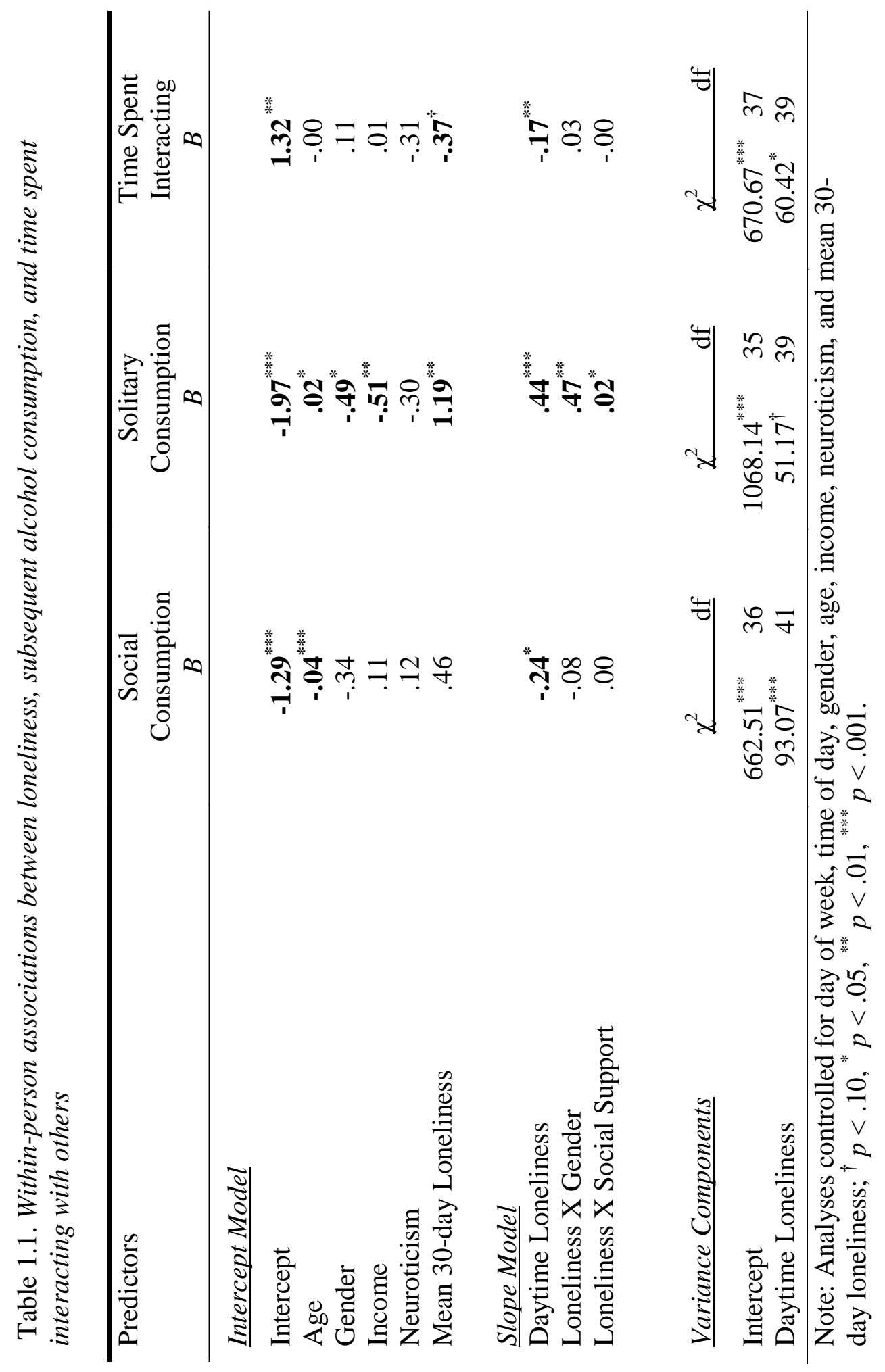


Table 1.2. Multilevel mediation models for social and solitary outcome.

Mediation Model

$\underline{\text { Loneliness-Interacting-Social }}$

$a$

$b$

$c$

Loneliness-Interacting-Solitary

$a$

$b$

$c^{\prime}$
Point Estimate (SE)

$-.03(.01)$

$.05(.01)$

$.002(.01)$

$$
\begin{aligned}
& \mathbf{- . 0 3}^{*}(.01) \\
& \text {-.01* (.003) }
\end{aligned}
$$$$
.01 *(.004)
$$

$\underline{\text { Indirect Effect }}$

Loneliness-Intx-Social

Loneliness-Intx-Solitary $\underline{\text { Avg. Random Indirect Effect }}$

$\mathbf{- . 0 0 1}(.001)$

$.0003(.001)$
95\% CI

Lower Upper

$-2.38 \mathrm{E}-03 \quad-8.746 \mathrm{E}-06$

$-8.72 \mathrm{E}-04 \quad 1.6 \mathrm{E}-03$

Note: Analyses controlled for day of week, time of day, gender, age, income, neuroticism, and mean 30day loneliness. Bold indirect effect point estimates are significant based on $95 \%$ confidence intervals. 
Figure 2.0. Perceived social support moderation for loneliness-solitary consumption associations.

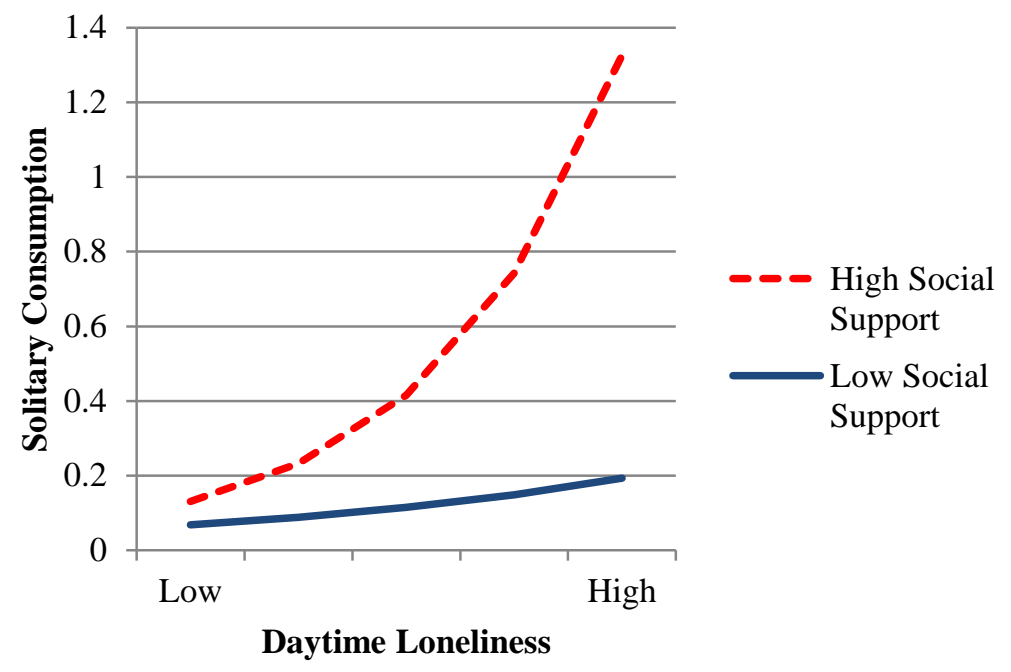


CHAPTER III. MANUSCRIPT II. NO ONE TO TURN TO IN TIMES OF JOY: LONELINESS AND CAPITALIZATION DEFICITS.

\begin{abstract}
Previous work has demonstrated that lonely persons are less likely to disclose negative events (e.g., Solano, Batton, \& Parish, 1982). Research has yet to explore how loneliness influences the disclosure of positive events, or capitalization (Langston, 1994). The current study examined how chronic loneliness relates to the likelihood of capitalizing on positive events within an undergraduate student sample $(\mathrm{N}=1147 ; 74 \%$ female $)$. Additionally, the mediating effects of cognitive and perceptual consequences of loneliness (i.e., self-esteem, social support, perceived event importance) were explored. Results provide evidence in support of loneliness-related deficits in the likelihood of disclosing positive events, which were partially explained by lower levels self-esteem and perceived event importance. Findings revealed potential mechanisms through which loneliness interferes with positive relationship processes, contributing to the maintenance of loneliness over time.
\end{abstract}

Keywords: Loneliness, self-disclosure, self-esteem, social support 
The distressing experience of loneliness is one which is consequential for individual health and well-being (Cacioppo, Hawkley, \& Berntson, 2003; Peplau \& Perlman, 1979). Indeed, much work has shown that loneliness relates to negative physiological health outcomes, including but not limited to greater morbidity, disease susceptibility, sleep deficits, and poor cardiovascular health (e.g., Cacioppo et al., 2003). Defined as perceived social isolation (Perlman \& Peplau, 1984), loneliness is a commonly-reported experience, which according to the cognitive-discrepancy model of loneliness, is based on a discrepancy between desired and actual levels of social contact (Paloutzien \& Janigian, 1987; Russell, Peplau, \& Cutrona, 1980). Thus, whereas objective levels of social isolation are certainly known to predispose individuals to this negative-affective experience (e.g., Cutrona, 1986; Hawkley et al., 2008), loneliness is distinct from objective social isolation as it is rooted in an evaluation of relationships rather than actual number of social ties. In turn, even the most socially-connected of individuals can experience loneliness. Indeed, recent polls suggest that $30-40 \%$ of Americans report feeling lonely at any given time (e.g., Bekhet \& Zauszniewski, 2012; Wilson \& Moulton, 2010). Further, despite increasing access to social contact which social media and the internet provides, it is suspected that rates of loneliness are on the rise (Cacioppo et al., 2003; de Jong-Gierveld, 1987), thus making this affective experience a timely and relevant construct to explore.

Early theorists describe loneliness as a multidimensional construct, with various affective, cognitive, and behavioral manifestations (Peplau \& Perlman, 1979). In support of this multidimensional framework, research has revealed various behavioral and 
perceptual correlates of loneliness which may be consequential to social well-being and the development of close relationships. In particular, evidence suggests that lonely persons exhibit poorer social skills (e.g., lower conversational involvement; Bell, 1985), greater social-withdrawal and lower self-esteem (e.g., Peplau \& Perlman, 1979, and report more negative perceptions of social relationships and social interactions in general (e.g., Wittenberg \& Reis, 1986). Transactional models of loneliness posit that these various social, perceptual, and behavioral consequences operate on the environment to maintain this negative-affective experience (Ernst \& Cacioppo, 1998). Otherwise stated, the various consequences or symptoms of loneliness likely inhibit positive social interactions, thus stunting the development of relationships, and as a result contributing to continued feelings of loneliness over time. Whereas previous studies examining this negative-affective experience have made mention of transactional models in explaining the persistent effects of loneliness (e.g., Ernst \& Cacioppo, 1998), few studies have actually tested mechanisms through which loneliness relates to behavioral outcomes. The current study examined the indirect effects of multiple social perceptual and cognitive correlates of chronic loneliness in inhibiting positive social behavior, namely the disclosure of positive events or capitalization.

\section{Self-Disclosure Deficits and Capitalization}

One particular social behavior which is known to play a large role in the development of interpersonal relationships, and which evidence has shown to be deficient among lonely persons, is self-disclosure. In general, lonely persons tend to withdraw socially in times of need rather than making use of social capital resources through 
support-seeking behavior (Cacioppo et al., 2000; Heinreich \& Gullone, 2006). Further, lonely persons are not only less likely to report disclosing negative events (Solano et al., 1982), but they also exhibit difficulty managing appropriate levels of disclosure during social interactions when they do occur (Chelune, Sultan, \& Williams, 1980). In line with transactional models of loneliness, researchers posit that these deficits in disclosure behavior can be at least partially explained by the social behavioral and perceptual deficits symptomatic of loneliness (e.g., social skill deficits, negative perceptions of social interactions; Rotenberg, 1997). Specifically, these deficits contribute to lonely person's avoidance of disclosure interactions and social interactions in general, thus thwarting opportunities for relationship development. Additionally, lonely persons may view disclosure as a risky behavior, such that it requires a certain level of vulnerability during social interactions (Wittenberg \& Reis, 1996). Yet, whereas the avoidance of disclosure behavior may be momentarily more comfortable among the lonely, it inhibits these individuals from reaping the social rewards of self-disclosure. As one of the building blocks of interpersonal relationships, disclosure behavior plays an important role in facilitating feelings of interpersonal trust, closeness, and connectedness (e.g., Collins $\&$ Miller, 1994). That lonely persons are missing out on this interpersonal process is problematic, given its potential to reduce loneliness through the facilitation of intimate relationships.

The majority of existing research relating loneliness to disclosure behavior, as well as more general research and theory on self-disclosure, has focused primarily on the disclosure of negative events, self-relevant information, and personal experiences (Reis \& 
Shaver, 1988; Solano et al., 1982). Yet, more recent research has identified the disclosure of positive events, or capitalization (Gable, Reis, Impett, \& Asher, 2004; Langston, 1994) as a particularly important relationship development and maintenance process. Some recent researchers have even asserted that the social sharing of positive events may be a more powerful predictor of well-being and relationship outcomes than the disclosure of negative events and stressors (e.g., Kashdan, Ferssizidis, Farmer, Adams, \& McKnight, 2013). Indeed, sharing good news with others (capitalization) is related to a variety of intrapersonal and interpersonal outcomes, including increased levels of positive affect, memorability of positive events, increased social support, feelings of intimacy, closeness, trust towards others, and increased life satisfaction (Gable, Gonzaga, \& Strachman, 2006). Further, when met with a supportive response, capitalization has been shown to reduce momentary feelings of loneliness (Gable \& Reis, 2010). The capitalization literature has demonstrated that the presence of others is just as vital during the good times and during the bad, and has thus contributed largely to the field of positive psychology and relationships science.

\section{A Positivity-Deficit Perspective}

Interestingly, in a recent review of the capitalization literature, Gable and Reis (2010) called for research considering person factors which may interfere with the ability to successfully engage in positive relationship processes, such as capitalization. Given that loneliness is related to deficits in the disclosure of negative events, as well as reduced support-seeking behavior in general, it is likely that chronically lonely persons also exhibit deficits in capitalization behavior. However, no research to date has examined 
chronic loneliness as one individual difference variable which influences capitalization behavior, thus thwarting the development of close relationships.

Recent work by Kashdan et al. (2013) examined deficits in capitalization behavior among individuals with social anxiety. In particular, Kashdan explores positivity deficits which interfere with socially anxious individuals' ability to develop and maintain healthy interpersonal relationships, thus contributing to dysfunctional behavior and psychological maladjustment, and relationship difficulties. Specifically, socially anxious persons report receiving a less supportive response from others when disclosing positive events, and rate positive events as less important and meaningful compared to their less socially anxious counterparts (Kashdan et al., 2013). Similar research has examined the behavior of low self-esteem individuals in relation to capitalization, showing that those with low selfesteem perceive their partners as less responsive, and thus benefit less from capitalization as a relationship-maintenance strategy (Smith \& Reis, 2012). The current study takes a positivity-deficit perspective by examining how loneliness uniquely relates to the selfreported disclosure of positive events.

Further, and in line with transactional models of loneliness, this study explores how various consequences and correlates of loneliness function to impede capitalization behavior, thus thwarting the development of close, meaningful relationships. Whereas transactional models of loneliness suggest that the various social behavioral and perceptual consequences of loneliness play role in inhibiting support-seeking behavior, the authors are aware of no research to date which has actually tested mechanisms through which loneliness relates to deficits in disclosure behavior. The current 
investigation addresses this gap in the literature by examining potential mechanisms through which loneliness might influence capitalization. Specifically, the extent to which self-esteem, social support, and perceptions of event importance contribute to lonelinessrelated deficits in the social sharing of positive events is explored (Figure 3.0).

Early work by Anderson, Horowitz, and French (1983) demonstrated that lonely persons attribute positive events and successes to external and temporary causes, whereas they attribute their failure to stable and internal-characterological deficits. Additionally, loneliness is related to greater negative affectivity (Davis, Hanson, Edson, \& Zielger, 1992). This work suggests that lonely persons tend to make more negative attributions for successes and positive events, and thus might be more likely to perceive positive events as less important relative to less lonely individuals. However, this attribution-style is problematic, in that it likely thwarts lonely individuals' ability to reap the benefits of positive experiences and successes, and may inhibit engagement in positive social interactions which celebrate these events. As described within Kashdan et al.'s (2013) positivity-deficit perspective, diminishing the importance of positive events may make individuals less likely to share them with others.

Further, existing research has provided support for self-esteem as a mediator of the potential relationship between loneliness and capitalization behavior. That is, selfesteem is one characteristic of chronically lonely persons which has previously been shown to relate to deficits in capitalization behavior (Perlman \& Peplau, 1981). Individuals with low self-esteem are not only less likely to utilize capitalization as a relationship maintenance and repair process, but they are less likely to be disclosed to, 
given others' expectations of unresponsive, unsupportive behavior (MacGregor \& Holmes, 2011; Smith \& Reis, 2012). Research by Wood, Heipel, and Michela (2003) further underscores this process by providing evidence that low self-esteem is associated with positive-affect dampening. That is, individuals with low self-esteem are less likely to exhibit positive-affect enhancing behavior, such as capitalization. To the extent that low self-esteem is a common characteristic among the lonely (e.g., Davis et al, 1992; McWhirter, 1997), it is possible that it plays a role in dampening lonely persons' responses to positive experiences via reductions in capitalization.

In addition to perceptions of event importance and self-esteem, it is also likely that general perceptions of support availability influence individuals' motivations to seek out others in celebration of positive experiences. Previous capitalization research has primarily examined social support as a key outcome of successful capitalization experiences (Gable \& Reis, 2010). Yet, as an indicator of expectations regarding the availability of close others, low perceptions of social support likely influence the likelihood that one will seek out others with whom to share positive experiences. However, no work to date has examined how general, trait-based levels of support influence the likelihood of capitalizing on positive events. Thus, the current study examined social support at a more trait-based level than is typically done in the capitalization literature, exploring how loneliness and related perceptions of support availability influence the likelihood of sharing positive events. In doing so, this investigation provides a novel perspective of social support, or perceived lack thereof, as 
one characteristic of loneliness which indirectly influences capitalization behavior, rather than an outcome of the process itself.

\section{Current Investigation}

The current study examined the inhibiting effects of loneliness on a key relationship maintenance and repair strategy, namely the disclosure of positive events. This study thereby makes a large contribution to the existing capitalization literature by exploring one person-factor (i.e., loneliness), which may interfere with individual's ability to successfully capitalize on positive events. Additionally, this study drew from established transactional models of loneliness (Ernst \& Cacioppo, 1998), to examine mechanisms through which loneliness relates to deficits in capitalization behavior (i.e., self-esteem, social-support, and perceived event importance; Figure 3.0).

Based on existing research and theory, loneliness was expected to relate to a lower likelihood of capitalizing on positive events. Further, it was anticipated that this relationship would be partially mediated by lower levels of self-esteem, perceived event importance, and perceptions of social support among lonely versus less lonely individuals. Whereas previous research has examined how loneliness relates to the disclosure of negative events, no studies to date have examined whether lonely people differ in socially sharing positive events, or capitalization. Consequently, the present investigation makes a substantial contribution to the current loneliness literature. To the extent that capitalization has various intrapersonal and interpersonal outcomes, including reduced feelings of loneliness in the moment, it was important to examine processes 
through which chronic levels of loneliness inhibit this positive relationship behavior, thus contributing to the persistence of loneliness over time.

\section{Methods}

\section{Participants}

One thousand one hundred and forty-seven undergraduate students (74\% female) were recruited via in-class announcements in undergraduate psychology courses. Participants were mostly non-traditional aged undergraduate students, compared to typical college student samples, with an average age of $26(S D=7.80$, range $=17-70)$. Sixty-two percent of participants were employed, with $65 \%$ earning over $\$ 16,000$ per year. Sixty-five percent of participants reported being single, 29\% married or cohabitating, $16 \%$ parents, $87 \%$ identified as heterosexual. Additionally, $65 \%$ of participants were Caucasian, 11\% Asian, 8\% Hispanic/Latino, 4\% African American, 2\% Native Hawaiian/Pacific Islander, 2\% Native American, and 8\% identified as Other. Participants were offered extra credit in compensation for their participation in the study.

\section{Procedures}

Following in-class advertisements of the study, interested and eligible participants were provided a link to an online informed consent followed by a 30-40 minute online survey. The questions of interest in the present study were assessed as part of a larger survey examining individual differences and retrospective reports of past week social and health behavioral experiences. Participants were told that the purpose of the study was to assess how social interactions and emotions affect engagement in certain behaviors. 


\section{Measures}

Chronic loneliness. Chronic loneliness was assessed via the UCLA Loneliness Scale (University of California; Russell, 1996). Participants were asked to respond to twenty statements relating to general feelings of belongingness using a 4-point Likert scale (1- Never, 4-Always). Example items included "How often do you feel that you are "in tune" with the people around you?" and "How often to you feel that your relationships with others are not meaningful." This scale demonstrated high internal consistency (Cronbach's alpha $=.94)$.

Capitalization. Capitalization and perceived event importance were assessed in terms of the past 7-day experiences of positive event disclosure. Participants were asked to think about the most positive thing that happened to them and to rate the importance of this event (1-Unimportant, 5-Important). Capitalization was assessed by a yes/no question, to which participants indicated whether or not they had told anyone about this event. These items were modeled after Gable et al.'s (2004) assessment of capitalization experiences and perceived event importance.

Perceived social support. A twelve-item version of the Interpersonal Support Evaluation List (ISEL; Cohen, Mermelstein, Kamarck, \& Hoberman, 1985) was used to assess general perceptions of support availability. Participations responded to items relating to the perceived availability of others in times of need using a four point scale (1Definitely true, 4-Definitely false). This scale is composed of three subscales assessing belonging, appraisal, and tangible support. Total social support was assessed by summing 
across the three subscales. Items demonstrated adequate internal consistency (Cronbach's alpha $=.82)$.

Self-esteem. Self-esteem was assessed by the Rosenberg Self-Esteem Scale (Rosenberg, 1965). Participants indicated their agreement with 10-items describing attitudes towards the self, using a 4-point rating scale (1-Strongly agree, 4-Strongly disagree). Sample items include "On the whole, I am satisfied with myself" and "I take a positive attitude toward myself." Negatively-framed items were recoded, and items were summed to create a composite self-esteem score, with higher scores representing greater self-esteem. This measure exhibited adequate internal consistency (Cronbach's alpha = $.90)$.

\section{Data Screening and Transformations}

Of the original 1147 participants, 238 were missing observations on key study variables. Further, nine additional cases were flagged as multivariate outliers. Exploratory analyses revealed that missingness of capitalization data was partially explained by levels of loneliness and social support. Interestingly, individuals higher in loneliness were less likely to have missing capitalization data $(r=-.06, p=.08)$. Participants with less social support were also less likely to have missing capitalization data $(r=.07, p=.05)$. Provided that no other patterns of missingness were detected in the data, missingness on these study variables qualified as missing at random (MAR;

Tabachnik \& Fidell, 2007) ${ }^{1}$. Given our large sample size and thus low concern for statistical power, the list-wise deletion method was used in addressing missing data. This method excludes cases for which a single value is missing on key study variables. These 
data screening procedures rendered a final sample size of $\mathrm{N}=909$ ( $80 \%$ of our original sample; $74 \%$ female).

To address deviations from normality among key study variables, log, squareroot, and reflected-inverse data transformations were utilized, and improvement in skewness was examined for each variable. Final analyses were conducted with logtransformed loneliness and reflected inverse of perceived event importance, which exhibited substantially less skew than raw scores ${ }^{2}$. For ease of interpretation, the mean and standard deviations for the raw scores of all study variables are presented throughout this manuscript. However, transformed variables for loneliness and perceived eventimportance were utilized in all analyses.

\section{Data Analytic Strategy}

Following a model fit comparison for full versus partial multiple mediational models using Mplus v. 7.11 data analytic software (Muthén \& Muthén, 2008), the function of self-esteem, social support, and perceived event importance were tested as mediators of the relationship between loneliness and the likelihood of capitalizing on a positive event in the previous week (Figure 3.0). Previous researchers have suggested the use of bootstrapping for the estimation of direct and indirect effects, particularly for nonnormally distributed outcome variables (Shrout \& Bolger, 2002). Additionally, Preacher and Hayes (2008) asserted that the boot-strapping offers various advantages to the estimation of indirect effects over the traditional approaches, including the absence of strict distributional assumptions of normality. Given the categorical nature of the outcome variable (capitalization attempt: yes/no), as well as the negative skew of 
capitalization attempt data, bias-corrected bootstrapping (resample $\mathrm{N}=5,000$ ) was utilized to estimate confidence intervals for total and specific indirect effects. Further, to account for the dichotomous nature of the primary outcome variable, a weighted least squares estimator (WLSMV) was utilized in all analyses. As this particular estimator outputs probit regression coefficients, regression parameter estimates were transformed into numeric probabilities for ease of interpretation, using methods suggested by Muthén (2011).

\section{Results}

\section{Descriptives}

Of our final sample $(\mathrm{N}=909)$, eighty-two percent of participants reported disclosing positive events over the previous week. On average, these events were rated as high in importance $(M=4.52, S D=.82$, range $=1-5)$. Further, $30 \%$ of participants reported that the most important person they shared this event with was a friend. Preliminary analyses revealed that $40 \%$ of individuals shared their event with a significant other, $24 \%$ a parent or sibling, and $3 \%$ a coworker. Levels of loneliness in the sample ranged from 20-75 $(M=42.39, S D=11.69)$, and on average, participants reported relatively high levels of social support $(M=26.16, S D=7.03$, range $=0-36)$, and selfesteem $(M=30.83, S D=5.57$, range $=12-40)$. Inspection of bivariate correlations revealed that loneliness significantly related to study variables in expected directions. Specifically, those with greater loneliness reported lower self-esteem, less perceived support, and rated past-week positive events as less important compared to their lesslonely counterparts (Table 2.0). There were no significant gender differences in 
loneliness, self-esteem, or perceptions of social support. However, men and women did differ in their ratings of event importance, at $t(880)=-3.30, p=.001$, such that men rated positive events in the previous week as less important $(M=4.29, S D=1.02$, range $=1-5)$, compared to women $(M=4.57, S D=.79$, range $=1-5)$.

\section{Model Fit}

First, model fit was tested for a partial multiple mediation model with indirect effects of self-esteem, perceived event importance, and social support, specifying a direct effect of loneliness on the likelihood of capitalizing on a positive event from the previous week (Figure 3.0). The model demonstrated adequate fit to the data $(\mathrm{CFI}=.98, \mathrm{TLI}=$ .95, RMSEA $=.06$, WRMR $=.85$; Table 2.1). Next, partial model fit was compared with that of a full mediational model by fixing the direct effect path from loneliness to pastweek capitalization attempt at zero. A full mediational model demonstrated significantly worse fit than a partial mediational model $(\mathrm{CFI}=.97, \mathrm{TLI}=.92, \mathrm{RMSEA}=.08$, WRMR $=1.27 ; X^{2}$ Difference Test $=9.74, p=.002$ ). To investigate the possibility of equivalent models, model fit was examined for an alternative partial mediational model wherein past week capitalization attempts (0-No, 1-Yes), predicted levels of loneliness indirectly through self-esteem, social-support, and perceived event importance. This model exhibited substantively poorer fit than our original hypothesized model $(\mathrm{CFI}=.92, \mathrm{TLI}=$ .74 , RMSEA $=.113$ ). Thus, unstandardized parameter estimates and bias-corrected confidence intervals were estimated for the original partial mediational model (Figure 3.1), with loneliness predicting capitalization indirectly through self-esteem, social support, and perceived event importance. 


\section{Multiple Mediation}

Loneliness was significantly and negatively related to self-esteem $(b=-27.53, p<$ $.001)$, perceived event importance $(b=-.37, p<.001)$, social support $(b=-1.64, p<$ $.001)$, and the likelihood of sharing a positive event in the previous week $(b=-1.48, p=$ .009). Specifically, participants at higher levels of chronic loneliness reported lower levels of self-esteem, perceived their relationships as less supportive, rated positive events from the previous week as less important, and were less likely to report disclosing their positive event to someone over the previous week. As displayed in Table 2.2, controlling for levels of loneliness, self-esteem $(b=.03, p<.05)$ and perceived-event importance $(b=.80, p<.001)$ related to greater likelihood of positive-event disclosure. Specifically, above and beyond the effects of loneliness, those with greater self-esteem and those who perceived their positive events as high in importance were more likely to capitalize on a positive event during the previous week. Perceived social support was not significantly related to capitalization when controlling for chronic loneliness.

As displayed in Table 2.3 and Figure 3.2, results of a partial multiple mediation model revealed significant total indirect effects of social support, self-esteem, and perceived event importance on the loneliness-capitalization associations $(Z=-1.16, p=$ .001 ; BC 95\% CI: $[-1.66,-.55])$. However, only the specific indirect effects of selfesteem $(Z=-.74, p=.012 ;$ BC 95\% CI: $[-1.21,-.25])$ and perceived event importance $(Z$ $=-.30, p=.001 ; \mathrm{BC} 95 \% \mathrm{CI}:[-.46,-.17])$ were significant. That is, loneliness related to a lower likelihood of sharing positive events in the previous week, primarily due to lower self-esteem and perceptions that these positive events were of less importance. 
As the estimated regression weights outputted by the WLSMV estimator are probit regression coefficients, primary parameter estimates were transformed into numeric probabilities. Further, to aid in the interpretation of model findings, probability profiles were computed, detailing the likelihood of capitalization at high and low levels of loneliness, given varying levels of self-esteem, social support, and event importance. This detailed descriptive information clearly demonstrates the extent to which loneliness thwarts engagement in capitalization behavior, as well as the contributing role of its various symptoms and correlates.

Probit regression coefficients were transformed into probabilities and probability profiles were computed using methods suggested by Muthén (2011),

$$
\begin{gathered}
P(u=1 \mid X)=F\left(-t+b_{1} * X+b_{2} * X+b_{3} * X \ldots\right) \\
P(u=0 \mid X)=1-F\left(-t+b_{1} * X+b_{2} * X+b_{3} * X \ldots\right)
\end{gathered}
$$

where $-t$ is the negated threshold value for the categorical outcome, $b_{1}-b_{2}$ are probit regression weights and $\mathrm{X}$ are selected values for predictors and covariates included in the model. Probabilities are computed by entering threshold, probit coefficients and predictor values into the equation. The resulting value is then compared to a z-distribution table from which the relevant probability is attained.

Using this method, probabilities of capitalizing on positive events for individuals at four different probability profiles were computed. Specifically, the probability of capitalizing on positive events for: (1) individuals at high levels of loneliness (+1 SD) and mean levels of self-esteem, social support, and perceived event-importance (Profile 1); (2) individuals at low levels of loneliness (-1 SD) and mean levels of self-esteem, 
social support, and perceived event-importance (Profile 2); (3) individuals at high levels of loneliness (+1 SD) and below mean levels (-1 SD) of social support, self-esteem, and perceived event importance (Profile 3); and (4) individuals at low levels of loneliness (-1 SD), and above mean levels (+1 SD) of social support, self-esteem, and perceived eventimportance (Profile 4).

As outlined in Table 2.4, results indicated that the probability of sharing a positive event for individuals at low levels of loneliness and mean levels of self-esteem, social support, and perceived event importance was $91 \%$, and $70 \%$ for individuals at high levels of loneliness and mean levels of self-esteem, social support, and perceived event importance. Additionally, for those at high levels of loneliness and below mean levels of self-esteem, social support, and event-importance, the probability of capitalizing in the previous week was $35 \%$, while it was $98 \%$ for those at low levels of loneliness, and above mean levels of social support, self-esteem, and perceived event importance. Otherwise stated, individuals at high levels of loneliness and at below mean levels of our proposed mediators (self-esteem, social support, perceived event importance) were more than two times less likely to report disclosing positive events in the previous week, compared to those at low levels of loneliness and above or at mean levels of self-esteem, social support, and perceived event importance (who capitalized on positive events over the previous week nearly $100 \%$ of the time). 


\section{Discussion}

The present study explored associations among chronic levels of loneliness and past week reports of positive-event disclosure, or capitalization. Previous work has primarily focused on more negative processes related to loneliness, such as maladaptive health outcomes (i.e., greater morbidity and mortality), risk-related behavior (i.e., alcohol consumption, illicit substance use; Shankur et al., 2011), and stress-related supportseeking behavior (i.e., negative-event disclosure; Solano et al., 1982). Yet, as demonstrated by recent research within the emotions and social relationships literature, positive and negative processes and behavioral systems are functionally independent. That is, the absence of negative does not imply the presence of positive, and vice versa (Reis \& Gable, 2003). Thus, the current study makes a significant contribution to the existing literature by examining loneliness in the context of an important positive relationship process, which plays a role in the development of relationships and thus reduction of loneliness over time.

Additionally, this study is in line with recent work examining positivity deficits which inhibit certain individuals from reaping the benefits of capitalization (e.g., Kashdan et al., 2013). Specifically, previous research has shown that certain traits, such as social anxiety, inhibit individuals from experiencing the potential rewards of positive social interactions, by interfering with the success of positive social processes when they do occur (i.e., responsive capitalization interactions). Our findings reveal similar positivity deficits among chronically lonely individuals, showing that loneliness is related to a much lower likelihood of capitalizing on positive events. Conversely, for individuals 
at lower levels of loneliness, capitalization was nearly universal, approaching $100 \%$ in our sample. Thus, persons high in loneliness appear to be missing out on an important relationship development and maintenance processes, which may be contributing to the persistence of loneliness over-time. Future research should consider how loneliness influences perceptions and behaviors within capitalization interactions when they do occur. Specifically, examining how lonely people perceive the behavior of interaction partners in response to capitalization attempts would provide insight into more specific mechanisms which interfere with lonely person's ability to reap the benefits of capitalization interactions. Additionally, whether lonely individuals exhibit supportive behavior to others' disclosure attempts would provide valuable insight into how loneliness inhibits successful engagement in supportive social interactions, such as disclosure, which are transactional and dyadic in nature (Clark \& Lemay, 2010). That is, theory suggests that perceiving others as responsive to disclosure attempts relates to the provision of responsiveness to others.

Future research should also consider whether nonlonely others disclose positive events to lonely individuals. Smith and Reis (2012) provide evidence that individuals re less likely to disclose positive events to those they perceive as low in self-esteem, as they anticipate that these individuals will respond unsupportively. Similar behaviors and perceptions may occur when individuals perceive their partners as high in loneliness (Rotenberg \& Kmill, 1992). Evidence suggests that providing responsive behavior to others' disclosure of positive events builds social resources for both the discloser and the listener (i.e., increased partner liking and enjoyment of the social interaction; Reis et al., 
2010). Similarly, being disclosed to often results in a boost in well-being, as it is indicative of others' openness and trust, as well as an individual's relational value (i.e., the extent to which in individual is viewed as an important and valuable relationship; Laurenceau, Feldman, Barrett, \& Pietromonaco, 1998; Leary, 2010). Thus, in being less likely to disclose positive events to others, lonely individuals may not only be missing the personal and social benefits of capitalization, but also the individual and social rewards of listening to and responding to others' capitalization on positive events.

In addition to establishing evidence suggestive of general positivity deficits related to loneliness (i.e., deficits in positive-event disclosure behavior) our findings revealed two potential mechanisms which may play a role in inhibiting lonely persons from capitalizing on positive events. In this sample, lonely persons were less likely to share positive events due to low levels of self-esteem, and because they did not view their positive experience as important as their less lonely peers. That self-esteem and perceived event importance were partial mediators of the relationship between loneliness and the likelihood of capitalizing on positive events is suggestive that these cognitive correlates of loneliness play a role in inhibiting positive social interactions. These findings provide support for theorized transactional models of loneliness, which posit that the various consequences of loneliness interact with the environment to maintain this affective experience (Ernst \& Cacioppo, 1998). Our results demonstrate one process through which this transaction may occur, namely by inhibiting engagement in capitalization as a relationship development process. 
Our findings also speak to research examining positive-affect dampening associated with low levels of self-esteem (Wood et al., 2003). In particular, Wood et al. (2003) provided evidence that individuals high in self-esteem report engaging in behaviors to prolong their positive affect, whereas the behavior of those low in selfesteem dampened levels of positive affect. To the extent that capitalization is a form of positive affect regulation, our findings build on this research in showing that lonely individuals, who tended to report lower levels of self-esteem, were less likely to engage in positive affect enhancement and savoring strategies (i.e., positive-event disclosure) in response to positive events. Future work should consider whether lonely individuals are actively dampening affective reactions to positive events (e.g., trying to feel not as good, distracting oneself, dampening excitement and trying to calm down; Wood et al., 2003), in addition to not disclosing these events with others.

Whereas our findings revealed significant total indirect effects, and significant specific indirect effects of self-esteem and perceived event-importance, the specific indirect effects of social support were not significant. As chronic loneliness and social support were strongly related in this sample $(r=-.75, p<.001)$, and as the two are closely related conceptually, it is likely that social support contributed little to the prediction of positive-event disclosure, over and above the effects of loneliness. Indeed, while perceived social support significantly and positively related to past week capitalization in our sample $(b=1.09, p<.01)$, this relationship was no longer significant when loneliness $(b=.54, p<.19)$ was included in the model. It is also possible that the measurement of perceived support was too general, and thus did not capture specific 
deficits in support (i.e., perceived responsiveness to capitalization) which might influence capitalization behavior. Additionally, the items asked in the assessment of social support tended to focus on the availability of others in times of need (i.e., "There is someone I can turn to for advice about handling problems with my family...”), which may not apply to the availability of others during times of joy.

An important construct which typically examined within the capitalization literature is the general perception of close others' responsiveness to capitalization attempts. Research has shown that the personal and social benefits of capitalization are contingent on whether an individual perceives others' response as validating, understanding, and caring (Maisel, Gable, \& Strachman, 2008). Though a distinct construct, perceived responsiveness is related to perceptions of social support. In fact, this construct has at times been referred to as capitalization support (Kashdan et al., 2013). Yet, current assessments of responsiveness within the context of capitalization tend to focus on perceptions of responsiveness within romantic relationships. As the primary focus of the current study was on loneliness and capitalization experiences within a larger sample of single and partnered individuals, perceived responsiveness was not included in the current analyses. Future research should consider whether general perceptions of responsiveness, in relation to both close and non-intimate others, relate to loneliness and thus capitalization behavior. That is, if lonely persons perceive that others are generally unsupportive and unenthusiastic (i.e., unresponsive) in response to shared positive events, they may be less likely to share these events with others altogether. Such 
research would capture yet another social-perceptual deficit related to loneliness which could potentially influence these individuals' ability to capitalize on positive events.

Though results of the current study reveal two potential mechanisms influencing the relationship between loneliness and the disclosure of positive events, tests of model fit indicated that a partial mediated model provided better fit to the data compared to a full mediational model. Thus, self-esteem and event-importance are just two among many other potential consequences of loneliness influencing capitalization behavior. More research is needed to determine other mechanisms through which loneliness could relate to positive-event disclosure. Specifically, future research should consider whether variables such as social anxiety, attachment, and general perceptions of responsiveness among partnered and unpartnered individuals (as described above) play a role in relating to loneliness-related deficits in capitalization behavior. Though various other mechanisms are left to be explored, this study provided an important first step in developing a positivity deficit model of loneliness. Specifically, the current study supports the conclusion that loneliness inhibits individuals from engaging in and reaping the rewards of positive social interactions, and interferes with processes essential for the development of close, meaningful social relationships.

\section{Limitations}

Though this study makes various contributions to existing loneliness research, there are multiple limitations which should be considered, in particular the use of a college student sample. Previous researchers have expressed concern with the utilization of college student samples, given limitations to generalizability of results to the larger 
population. However, the current sample was drawn from a largely non-traditional aged undergraduate population (mean age $=26$, range 17-70 years old). Additionally, a sizable percentage of our sample reported diverse life experiences (i.e., 29\% married or cohabitating; $16 \%$ parents) which are typically not observed in traditional-aged undergraduate student samples. Thus, participants in this study are unique from traditional student samples, rendering our findings are potentially more generalizable to the larger population.

It should also be noted that participants in this sample exhibited a diverse spread of loneliness scores (range $=20-75$ ). Additionally, average levels of loneliness in this sample $(M=42.39, S D=11.69)$, were comparable to previous studies of loneliness among college students (Russell et al., 1996). Average levels of self-esteem $(M=30.83$, $S D=5.57)$ and social support $(M=26.16, S D=7.03)$ were also comparable to scores observed in previous work with normative adult samples (self-esteem, $M=32.62, S D=$ 5.80, Sinclair et al., 2010; social support, $M=28.80, S D=5.7$, Cohen et al., 1985). Thus, our findings are generalizable to normative samples, particularly in relation to levels of loneliness, self-esteem, and social support. Additionally, in calculating and presenting numerous probability profiles, findings are generalizable to individuals at varying degrees of loneliness, and at different levels of self-esteem, social support, and perceived event importance.

Of additional concern is the gender imbalance in our sample ( $74 \%$ female). In particular it is possible that our findings are descriptive of a predominantly female phenomenon. Yet, the only gender difference among primary study variables was in 
perceived event importance, for which women perceived their positive events from the previous week as of greater importance compared to men. Interestingly, the effect size for this difference was relatively small $\left(\eta^{2}=.02\right)$, and thus is likely due to the gender imbalance in the data (74\% female), skew in the event importance variable, and large sample size $(\mathrm{N}=909$ [882 reported gender]). Research should consider relationships among loneliness, self-esteem, social support, perceived event importance, and past week capitalization within a more balanced sample, testing for gender differences in indirect effects and examining different mechanisms which may be at play for male versus female participants.

In addition to the gender imbalance, there was little variability in reports of past week capitalization attempts $(82 \%$ of participants reported telling someone about their positive event from the previous week). However, the high frequency of capitalization attempts in this sample is consistent with the previous capitalization literature, which has shown that people tend to disclose the most positive events that happen to them $60-80 \%$ of the time (Gable \& Reis, 2010). Further, this study makes a large contribution by revealing differences in capitalization for individuals at high levels of loneliness. As demonstrated in the calculation of probability profiles, those at high levels of loneliness demonstrated far lower levels of capitalization, compared to those at low levels of loneliness and high levels of social support, self-esteem, and event important. Thus, whereas existing literature has provided evidence that capitalization is a commonly reported behavior, results of the current study demonstrate that this may be limited to 
individuals at low levels of loneliness, who may be more likely to perceive others as available in times of joy and draw from these support resources accordingly.

Another potential limitation is the possibility of alternative explanations for the relationships observed in the current study. Of note is the role of depression, a strong correlate of loneliness, which is related to similar patterns of attributions for positive and negative events. Though loneliness and depression are distinct constructs, depression was not measured in the current study. Thus, the influence of this construct on the observed associations cannot be explored, nor can the potential effects of depression be controlled for in the current study. However previous work has described loneliness as a unique risk factor for depression, the reciprocal effects of which unfold over time to predict greater depressive symptomology and loneliness (Cacioppo, Hughes, Waite, Hawkley, \& Thisted, 2006). Thus, it is possible that depression is another mechanism which helps to explain loneliness-related deficits in positive-event disclosure, or disclosure behavior more generally. Future work should assess the role of loneliness and depression in jointly predicting disclosure behavior, as well as attributional processes related to the celebration of positive events, which may be similar or distinct for these two constructs.

An additional limitation relates to issues of internal validity and directionality in our use of cross-sectional survey methods. Though survey methods are efficient in collecting large samples of data with the advantage of external validity, all of our analyses are correlational in nature and thus causal relationships among loneliness and capitalization cannot be established. Further, conclusions cannot be make regarding the extent to which loneliness leads to lower self-esteem and social support, ratings of event 
importance, and a lower likelihood of capitalizing on positive events, or whether these constructs somehow contribute to greater feelings of loneliness. In an investigation of equivalent models, the fit of a model wherein capitalization predicted loneliness indirectly through self-esteem, social support, and perceived event importance was explored. This model exhibited much poorer fit than our original hypothesized model, wherein loneliness predicted past week reports of positive-event disclosure.

Thus, although firm conclusions about the directionality or causal nature of associations observed in this study cannot be made, results provide initial support for the direction of relationships specified in our hypothesized model. To establish more definite evidence for causal links among loneliness and resulting capitalization experiences, future work should consider using experimental methods and loneliness manipulation. This would allow for the direct examination of how loneliness uniquely relates to deficits in perceptions of and behaviors during social interactions, which likely make even transient experiences of loneliness difficult to overcome.

\section{Conclusion}

The experience of loneliness is one which is consequential for individual health and well-being (Cacioppo et al., 2003; Peplau \& Perlman, 1979). The current study extends these consequences of loneliness to include positivity deficits in interpersonal behavior and relationship development processes. Specifically, the results from this study reveal that chronic loneliness relates to a lower likelihood of capitalizing on positive events with others over the previous week. Additionally, this relationship was partially explained by lower levels of self-esteem and perceptions that these positive events were 
of low importance. That the lonely appear to be missing out on the significant personal and social benefits derived from capitalization provides insight into one process through which loneliness could be a particularly difficult emotional experience to overcome. In particular, loneliness and its various cognitive consequences and correlates appear may thwart important positive social interactions which could actually reduce levels of loneliness and aid in the development and maintenance of close interpersonal relationships over time. 


\section{References}

Anderson, C. A., Horowitz, L. M., \& French, R. D. (1983). Attributional-style of lonely and depressed people. Journal of Personality and Social Psychology, 45(1), 127.

Bekhet, A. K., \& Zauszniewski, J. A. (2012). Mental health of elders in retirement communities: is loneliness a key factor? Archives of Psychiatric Nursing, 26(3), 214-224.

Bell, R. A. (1985). Conversational involvement and loneliness. Communications Monographs, 52(3), 218-235.

Cacioppo, J. T., Ernst, J. M., Burleson, M. H., McClintock, M. K., Malarkey, W. B., Hawkley, L. C., Kowalewski, R. B., Paulsen, A., Hobson, J. A., Hugdahl, K., Spiegel, D., \& Berntson, G. G. (2000). Lonely traits and concomitant physiological processes: the MacArthur social neuroscience studies. International Journal of Psychophysiology, 35(2), 143-154.

Cacioppo, J. T., Hawkley, L. C., \& Berntson, G. G. (2003). The anatomy of loneliness. Current Directions in Psychological Science, 12(3), 71-74.

Cacioppo, J. T., Hughes, M. E., Waite, L. J., Hawkley, L. C., \& Thisted, R. A. (2006). Loneliness as a specific risk factor for depressive symptoms: cross-sectional and longitudinal analyses. Psychology and Aging, 21(1), 140.

Chelune, G. J., Sultan, F. E., \& Williams, C. L. (1980). Loneliness, self-disclosure, and interpersonal effectiveness. Journal of Counseling Psychology, 27(5), 462.

Clark, M. S., \& Reis, H. T. (1988). Interpersonal processes in close relationships. Annual Review of Psychology, 39(1), 609-672. 
Cohen, S., Mermelstein, R., Kamarck, T., \& Hoberman, H. M. (1985). Measuring the functional components of social support. In I. G. Sarason \& B. Sarason (Eds.), Social support: Theory, research and applications (pp. 73-94). The Hague, The Netherlands: Martinus Nijhoff.

Collins, N. L., \& Miller, L. C. (1994). Self-disclosure and liking: a meta-analytic review. Psychological Bulletin, 116(3), 457.

Cronbach, L. J. (1951). Coefficient alpha and the internal structure of tests. Psychometrika, 16(3), 297-334.

Cutrona, C. E. (1986). Objective determinants of perceived social support. Journal of Personality and Social Psychology, 50(2), 349-355.

Davis, S. F., Hanson, H., Edson, R., \& Ziegler, C. (1992). The relationship between optimism-pessimism, loneliness, and level of self-esteem in college students. College Student Journal, 26(2), 244-247.

de Jong-Gierveld, J. (1987). Developing and testing a model of loneliness. Journal of Personality and Social Psychology, 53(1), 119.

Ernst, J. M., \& Cacioppo, J. T. (1998). Lonely hearts: Psychological perspectives on loneliness. Applied and Preventive Psychology, 8, 1-22.

Gable, S. L., \& Reis, H. T. (2010). Good news! Capitalizing on positive events in an interpersonal context. Advances in Experimental Social Psychology, 42, 195-257.

Gable, S. L., Gonzaga, G. C., \& Strachman, A. (2006). Will you be there for me when things go right? Supportive responses to positive event disclosures. Journal of Personality and Social Psychology, 91(5), 904. 
Gable, S. L., Reis, H. T., Impett, E. A., \& Asher, E. R. (2004). What do you do when things go right? The intrapersonal and interpersonal benefits of sharing positive events. Journal of Personality and Social Psychology, 87(2), 228.

Hawkley, L. C., Hughes, M. E., Waite, L. J., Masi, C. M., Thisted, R. A., \& Cacioppo, J. T. (2008). From social-structural factors to perceptions of relationship quality and loneliness: The Chicago health, aging, and social relations study. The Journal of Gerontology Series B: Psychological Sciences and Social Sciences, 63(6), 375384.

Heinriech, L. M., \& Gullone, E. (2006). The clinical significance of loneliness: A literature review. Clinical Psychology Review, 26(6), 695-718.

Kashdan, T. B., Ferssizidis, P., Farmer, A. S., Adams, L. M., \& McKnight, P. E. (2013). Failure to capitalize on sharing good news with romantic partners: Exploring positivity deficits of socially anxious people with self-reports, partner-reports, and behavioral observations. Behavior Research and Therapy, 51, 656-668.

Langston, C. A. (1994). Capitalizing on and coping with daily-life events: Expressive responses to positive events. Journal of Personality and Social Psychology, 67(6), 1112.

Laurenceau, J. P., Barrett, L. F., \& Pietromonaco, P. R. (1998). Intimacy as an interpersonal process: the importance of self-disclosure, partner disclosure, and perceived partner responsiveness in interpersonal exchanges. Journal of Personality and Social Psychology, 74(5), 1238. 
Leary, M. R. (2010). Affiliation, acceptance, and belonging: The pursuit of interpersonal connection. In S. T. Fiske, D. T. Gilbert, \& G. Lindzey (Eds.), Handbook of social psychology, $5^{\text {th }}$ edition (Vol. 2, pp. 864-897). Hoboken, NJ: Wiley \& Sons.

MacGregor, J. C., \& Holmes, J. G. (2011). Rain on my parade: Perceiving low selfesteem in close others hinders positive self-disclosure. Social Psychological and Personality Science, 2(5), 523-530.

Maisel, N. C., Gable, S. L., \& Strachman, A. (2008). Responsive behaviors in good times and in bad. Personal Relationships, 15(3), 317-338.

McWhirter, B. T. (1997). Loneliness, learned resourcefulness, and self-esteem in college students. Journal of Counseling and Development, 75(6), 460-469.

Meyers, L. S., Gamst, G., \& Guarino, A. J. (2003). Applied multivariate research. Design and interpretation. Thousand Oaks, CA: Sage Publications, Inc.

Muthén, B. (2011). Applications of causally defined direct and indirect effects in mediation analysis using SEM in Mplus. Unpublished working paper, www.statmodel.com.

Muthén, L. K., \& Muthén, B. O. (2008). Mplus (Version 5.1). Los Angeles, CA: Muthén \& Muthén.

Paloutzian, R. F., \& Janigian, A. S. (1987). Models and method in loneliness research: Their status and direction. In M. Hogat \& R. Crandall. (Eds). Loneliness: Theory, research, and applications. [Special issue]. Journal of Social Behavior and Personality, 2(2), 31-36. 
Peplau, L. A., \& Perlman, D. (1979). Blueprint for a social psychological theory of loneliness. Love and Attraction, 101-110.

Perlman, D., \& Peplau, L. A. (1981). Toward a social psychology of loneliness. Personal Relationships, 3, 31-56.

Perlman, D., \& Peplau, L. A. (1984). Loneliness research: A survey of empirical findings. In L.A. Peplau \& S. Goldston (Eds.), Preventing harmful consequences of severe and persistent loneliness, (pp. 13-46). U.S. Government Printing Office: DDH Publication No. (ADM) 84-1312.

Preacher, K. J., \& Hayes, A. F. (2008). Asymptotic and resampling strategies for assessing and comparing indirect effects in multiple mediator models. Behavior Research Methods, 40(3), 879-891.

Reis, H. T., \& Gable, S. L. (2003). Toward a positive psychology of relationships. In C. L. M. Keyes, J. Haidt (Eds.), Flourishing: Positive psychology and the life welllived (pp. 129-159). Washington, DC, US: American Psychological Association.

Reis, H. T., \& Shaver, P. (1988). Intimacy as an interpersonal process. In S. Duck, E. F. Hay, D.F., S. E. Hobfoll, W. Ickes, B. M. Montgomery (Eds), Handbook of personal relationships: Theory, research and interventions, (pp. 367-389). Oxford, England: John Wiley \& Sons.

Reis, H. T., Smith, S. M., Carmichael, C. L., Caprariello, P. A., Tsai, F. F., Rodrigues, A., \& Maniaci, M. R. (2010). Are you happy for me? How sharing positive events with others provides personal and interpersonal benefits. Journal of Personality and Social Psychology, 99(2), 311. 
Rosenberg, M. (1965). The measurement of self-esteem. Society and the adolescent selfimage, 297, 307.

Rotenberg, K. J. (1997). Loneliness and the perception of the exchange of disclosures. Journal of Social and Clinical Psychology, 16(3), 259-276.

Rotenberg, K. J., \& Kmill, J. (1992). Perception of lonely and nonlonely persons as a function of individual differences in loneliness. Journal of Social and Personal Relationships, 9(2), 325-330.

Russell, D., Peplau, L. A., \& Cutrona, C. E. (1980). The revised UCLA loneliness scale: Concurrent and discriminant validity evidence. Journal of Personality and Social Psychology, 39(3), 472-480.

Shankur, A., McMunn, A., Banks, J., \& Steptoe, A. (2011). Loneliness, social isolation, and behavioral and biological health indicators in older adults. Health Psychology, 30(4), 377-385.

Shrout, P. E., \& Bolger, N. (2002). Mediation in experimental and non-experimental studies: new procedures and recommendations. Psychological Methods, 7(4), 422.

Sinclair, S. J., Blais, M. A., Gansler, D. A., Sandberg, E., Bistis, K., \& LoCicero, A. (2010). Psychometric properties of the Rosenberg Self-Esteem Scale: overall and across demographic groups living within the United States. Evaluation and the Health Professions, 33(1), 56-80.

Smith, S. M., \& Reis, H. T. (2012). Perceived responses to capitalization attempts are influenced by self-esteem and relationship threat. Personal Relationships, 19(2), $367-385$. 
Solano, C. H., Batten, P. G., \& Parish, E. A. (1982). Loneliness and patterns of selfdisclosure. Journal of Personality and Social Psychology, 43(3), 524.

Tabachnick, B. G., Fidell, L. S., \& Osterlind, S. J. (2001). Using Multivariate Statistics, $5^{\text {th }}$ ed. Boston, MA: Pearson Education, Inc.

Wilson, C., \& Moulton, B. (2010). Loneliness among older adults: A national survey of adults 45+. Prepared by Knowledge Networks and Insight Policy Research. Washington, DC: AARP. Nonartists, (45).

Wittenberg, M. T., \& Reis, H. T. (1986). Loneliness, social skills, and social perception. Personality and Social Psychology Bulletin, 12(1), 121-130.

Wood, J. V., Heimpel, S. A., \& Michela, J. L. (2003). Savoring versus dampening: Selfesteem differences in regulating positive affect. Journal of Personality and Social Psychology, 85(3), 566. 


\section{Footnotes}

${ }^{1}$ According to Tabachnik and Fidell (2007), data is missing at random (MAR) when the probability of missingness is dependent on values of other variables in the data set. Meyers, Gamst, and Guarino (2006) posit that data missing at random (MAR) can be considered ignorable missing data, and standard data deletion and analysis procedures can be used.

${ }^{2}$ Model fit was also tested for a model with untransformed, raw variables. Transformed data exhibited slightly better model fit, and so these findings were retained and reported. 


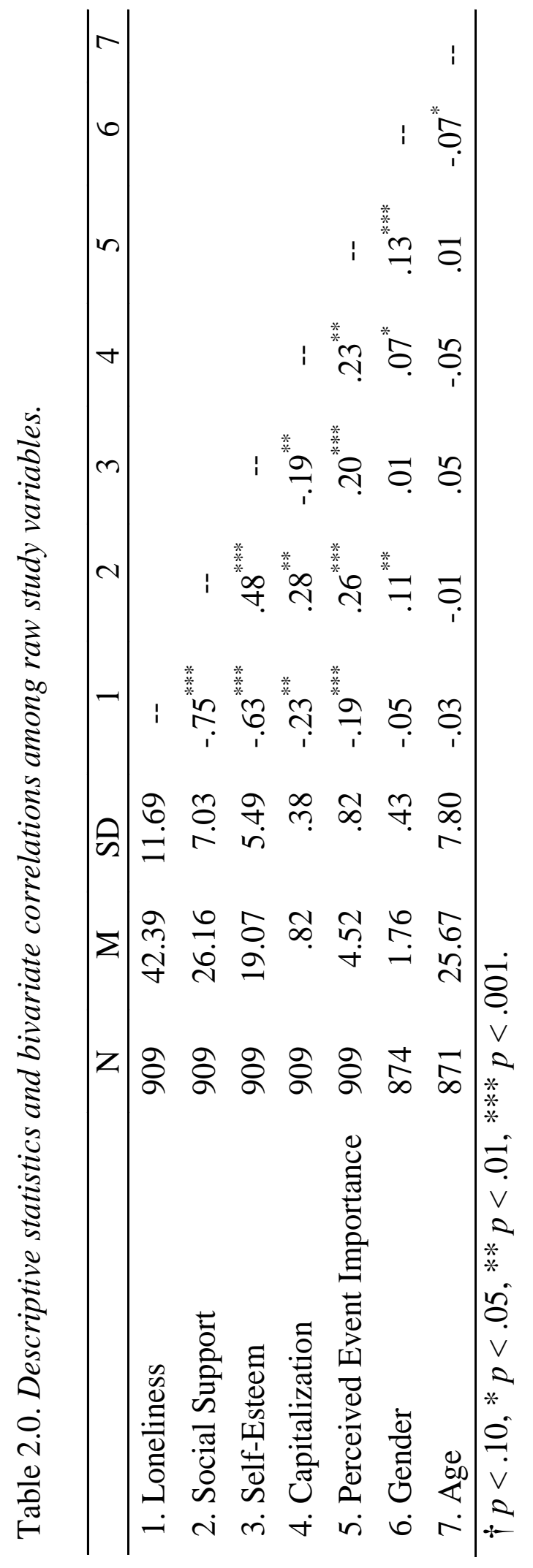


Table 2.1. Model fit and model comparisons for full and partial mediated models.

\begin{tabular}{|c|c|c|c|c|c|c|c|}
\hline \multicolumn{1}{|c|}{ Models } & $\boldsymbol{X}^{2}$ & df & TLI & CFI & RMSEA & WRMR & $\boldsymbol{X}^{2}$-Difference Test \\
\hline 1. Partially Mediated Model & $12.24^{*}$ & 3 & .95 & .99 & .06 & .85 & -- \\
\hline . Fully Mediated Model & $27.06^{*}$ & 4 & .92 & .97 & .08 & 1.27 & $9.73^{*}$ \\
\hline
\end{tabular}

Note: TLI $=$ Tucker-Lewis index, $\mathrm{CFI}=$ comparative fit index, RMSEA $=$ root mean square error approximation, $\mathrm{WRMR}=$ weighted root mean square residual; $\uparrow p<.10, * p<.01$. 
Table 2.2. Results for partial mediated model with transformed variables.

\begin{tabular}{|c|c|c|c|c|}
\hline \multirow[b]{2}{*}{ Parameter } & \multirow[b]{2}{*}{ Unstandardized $B$} & \multirow[b]{2}{*}{ SE } & \multicolumn{2}{|c|}{$95 \%$ BC CI } \\
\hline & & & Lower & Upper \\
\hline 1.Self-Esteem ON Loneliness & $-27.53^{* 2}$ & 1.19 & -29.41 & -25.55 \\
\hline 2. Event-Importance ON Loneliness & $-.37^{\prime \prime}$ & .07 & -.49 & -.25 \\
\hline 3. Social Support ON Loneliness & $-1.64^{* *}$ & .25 & -2.06 & -1.25 \\
\hline
\end{tabular}

Capitalization Attempt ON

\begin{tabular}{l|c|c|c|c}
\hline 4. Loneliness & $-1.49^{* *}$ & .57 & -2.45 & -.58 \\
\hline 5. Self-Esteem & $.03^{* *}$ & .01 & .01 & .04 \\
\hline 6. Social Support & .05 & .06 & -.05 & .14 \\
\hline 7. Event-Importance & $.80^{* * * *}$ & .17 & .51 & 1.07 \\
\hline
\end{tabular}

$* p<.05, * * p<.01, * * * p<.001$; Note: Zero within $95 \%$ BC CI indicates non-significance. 
Table 2.3. Total and specific indirect effects and bias-corrected confidence intervals.

\begin{tabular}{|c|c|c|c|}
\hline \multirow[b]{2}{*}{ Indirect Effects } & \multirow[b]{2}{*}{ Point Estimate } & \multicolumn{2}{|c|}{$95 \%$ BC CI } \\
\hline & & Lower & Upper \\
\hline 1. Total Indirect Effect & -.13 & -1.66 & -.55 \\
\hline 2. Self-Esteem & -.09 & -1.21 & -.25 \\
\hline 3. Perceived Event Importance & -.04 & -.46 & -.17 \\
\hline 4. Social Support & -.01 & -.25 & .07 \\
\hline
\end{tabular}

Note: Zero within 95\% BC CI indicates non-significance; bolded point estimates are statistically significant indirect effects. 
Table 2.4. Probability profiles for the likelihood of capitalizing on positive events.

\begin{tabular}{|c|c|c|}
\hline Probability Profile & $\begin{array}{c}\text { High loneliness } \\
\text { (+1 SD) }\end{array}$ & $\begin{array}{c}\text { Low Loneliness } \\
\text { (-1 SD) } \\
\end{array}$ \\
\hline $\begin{array}{l}\text { Above mean levels of self-esteem, social } \\
\text { support, and perceived event importance } \\
(+1 \mathrm{SD})\end{array}$ & -- & $\begin{array}{l}\text { Profile \#4 } \\
98 \%\end{array}$ \\
\hline $\begin{array}{l}\text { Mean levels of self-esteem, social } \\
\text { support, and perceived event importance }\end{array}$ & $\begin{array}{l}\text { Profile \#1 } \\
\quad 70 \%\end{array}$ & $\begin{array}{l}\text { Profile \#2 } \\
\quad 91 \%\end{array}$ \\
\hline $\begin{array}{l}\text { Below mean levels of self-esteem, social } \\
\text { support, and perceived event importance } \\
\text { (-1 SD) }\end{array}$ & $\begin{array}{l}\text { Profile \#3 } \\
\text { 35\% }\end{array}$ & -- \\
\hline
\end{tabular}

Note: Profiles were not computed for combinations of variables which were less likely to be representative of population members high versus low in loneliness (e.g., high loneliness, above mean levels of selfesteem, social support, and perceived event importance). 
Figure 3.0. Conceptual model of hypothesized relationships.

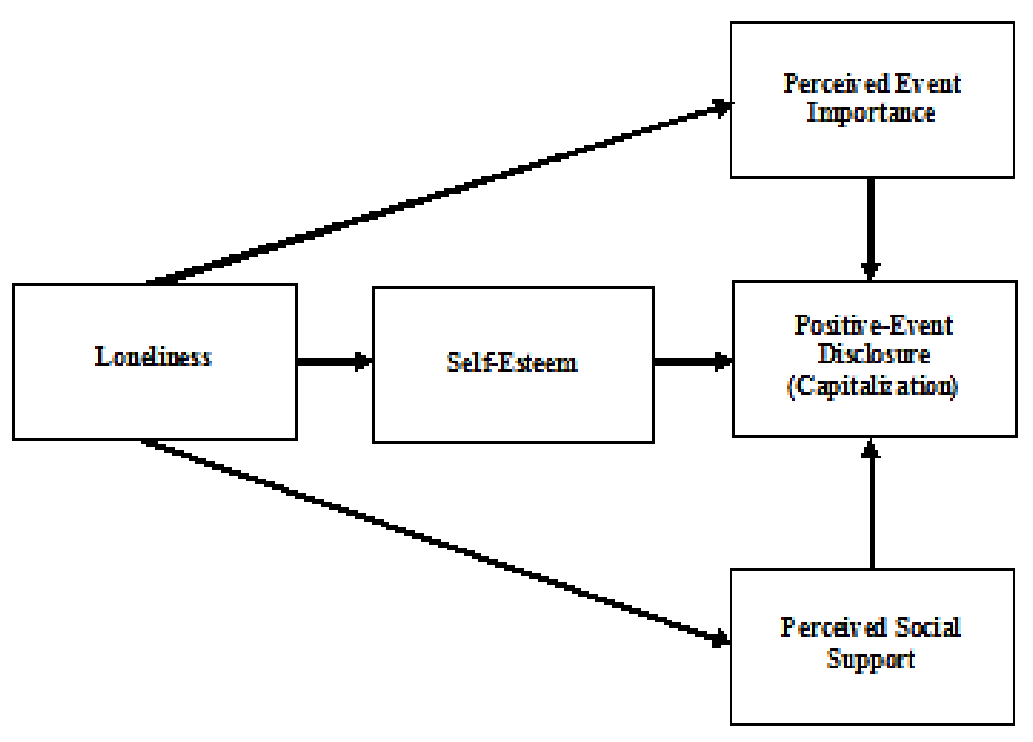


Figure 3.1. Partial-mediated model (final model).

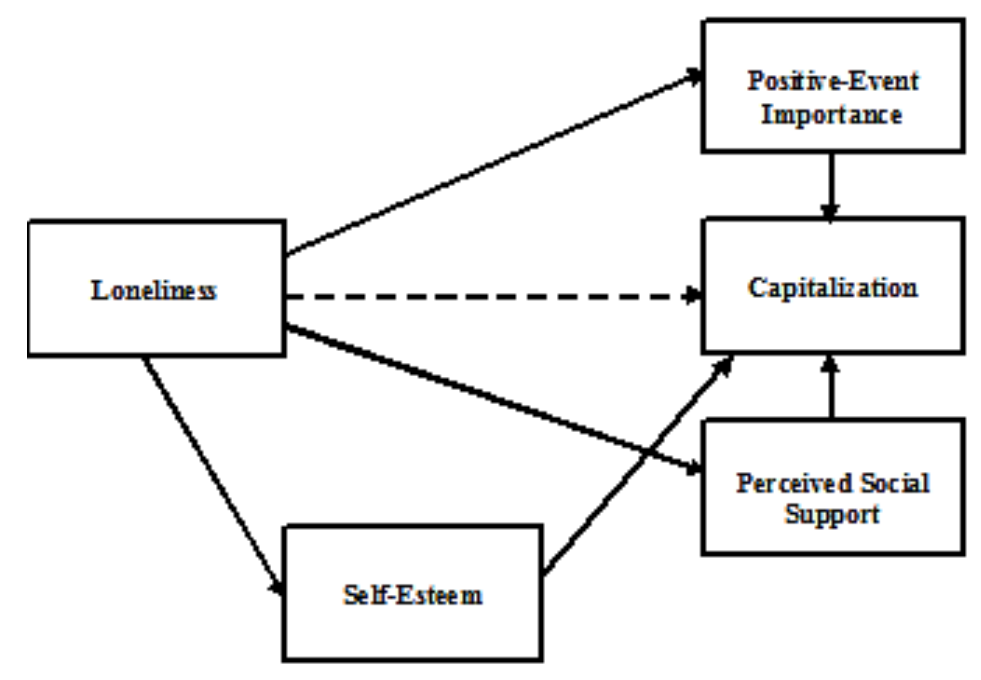


Figure 3.2. Partial-mediated model with parameter estimates.

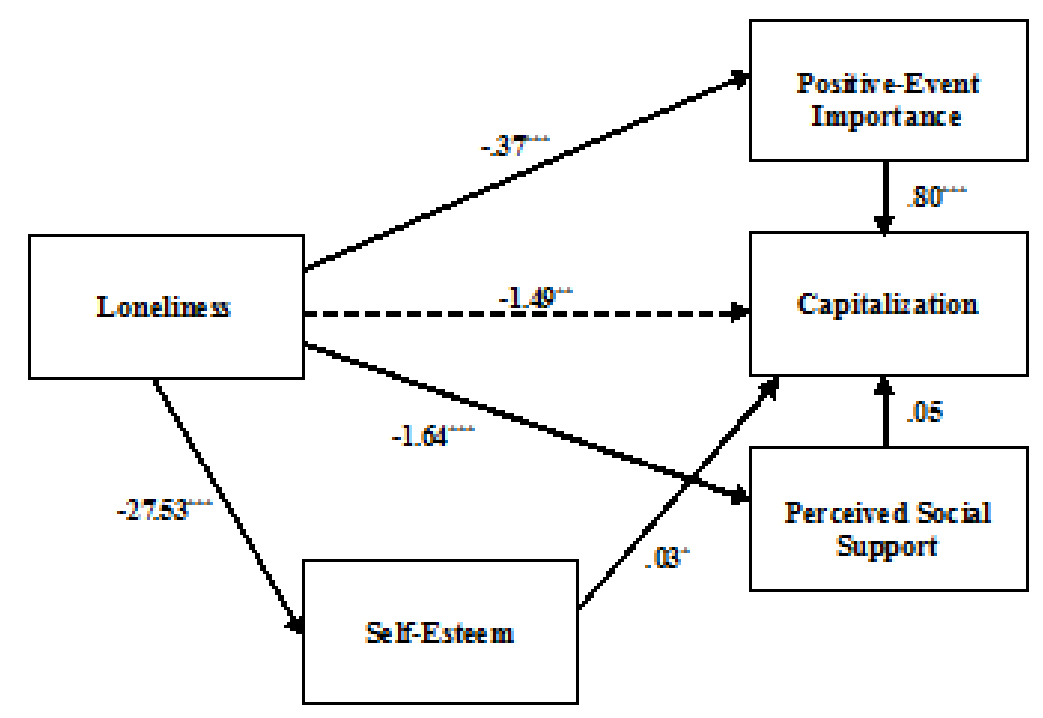


CHAPTER IV. MANUSCRIPT III: EXPERIMENTAL INDUCTION OF LONELINESS AND THE PERCEIVED PROVISION AND RECEIPT OF RESPONSIVE BEHAVIOR DURING POSITIVE-EVENT DISCLOSURE

\begin{abstract}
Previous research affirms that loneliness is a distressing experience, related to various social-perceptual and behavioral deficits (Perlman \& Peplau, 1979). Research examining the consequences of loneliness has been largely correlational, predominantly focusing on chronic loneliness. Thus, little is known about the role of transient loneliness within social interactions. Further, no research to date has considered the role of transient loneliness within positive social interactions (e.g., positive-event disclosure), which may have important implications for relationship development. The current study investigated causal links among transient loneliness and individual and interaction partner perceptions during a positive-event disclosure interaction, within a college student sample $(\mathrm{N}=166)$. 'Lonely' participants and interaction partners reported less positive affect and enjoyment following the event-sharing interaction. Additionally, 'lonely' participants perceived shared positive experiences as lower in importance. Moderated mediation revealed that individuals at lower levels of chronic loneliness experienced greater consequences of state loneliness in relation to the intrapersonal and interpersonal rewards of the capitalization (e.g., less enjoyment, positive affect, and partner liking). Results provide insight into social consequences of transient loneliness which may negatively impact individuals' ability to reap the benefits of positive social interactions. Keywords: Loneliness, capitalization, interpersonal communication
\end{abstract}


Humans have a fundamental motivation to form and maintain lasting, positive, and significant interpersonal relationships (Baumeister \& Leary, 1995; Deci \& Ryan, 2000). This universal tendency has affective consequences, results in pathological outcomes (psychological and behavioral) when thwarted, and elicits goal-directed behavior (Sheldon \& Gunz, 2009). Loneliness, or perceived social isolation, is one affective consequence of the impeded need to belong. Associated with feelings of dissatisfaction, unhappiness, anxiety, hostility, emptiness, boredom, and restlessness (Perlman \& Peplau, 1984), loneliness is a commonly reported affective experience, with $40 \%$ of Americans reporting feeling lonely at any given time (Bekhet \& Zauszniewski, 2012; Wilson \& Moulton, 2010). As defined by the cognitive-discrepancy model, loneliness is a distress that occurs when there is a discrepancy between actual and desired social relationships (Cacioppo et al., 2006). Thus, loneliness is distinct from objective levels of social isolation, and can be experienced by even the most socially-connected individuals.

Much work has considered the psychological and behavioral consequences of loneliness, providing evidence that long-lasting forms of loneliness (i.e., chronic loneliness) are predictive of poorer physical and psychological health, as well as riskrelated behavior (i.e., low rates of exercise and greater alcohol consumption; Glaser, Kiecolt-Glaser, Speicher, \& Holliday, 1985; Heinrich \& Gullone, 2006; Ouellet \& Joshi, 1986). In addition, lonely individuals tend to exhibit poorer social skills, socialwithdrawal, social anxiety, low self-esteem, and hold more negative perceptions of social interactions and close relationships (Peplau \& Perlman, 1979). Additionally, loneliness is 
related to lower levels of social support, higher anger, lower optimism, lower positive mood, greater negative mood, and greater shyness (Cacioppo \& Patrick, 2008). Lonely persons are typically perceived by others as less responsive and involved during social conversations, and are rated as less interpersonally attractive and less desirable as future friends (Bell, 1985; Jones, Hobbs, \& Hockenbury, 1982).

Early theorists distinguished distinct forms of loneliness, which differ in terms of chronicity and stability (e.g., Young, 1982). Specifically, loneliness can occur as a longlasting, pathological trait (i.e., chronic loneliness), or as a negative emotional state (i.e., transient loneliness). Whereas chronic experiences of loneliness are related to depression, transient loneliness is a more commonly reported experience, which fluctuates based on social interactions and the social context (Gross, Juvenon, \& Gable, 2002). Indeed, transient loneliness can be experienced by socially-connected individuals, and recent trends show that $20-40 \%$ of individuals in the United States report being lonely at any given time (e.g., Bekhet, \& Zauszniewski, 2012). Interestingly, existing research tends to focus on more chronic or prolonged levels of loneliness, such as those experienced among the clinically depressed. As such, less is known about the social-behavioral and perceptual processes related to transient or state-levels of loneliness. Further, little is known about mechanisms through which transient levels of loneliness could develop into more chronic levels over time. The current study makes a substantive contribution to the current literature by focusing on transient or state experiences of loneliness, particularly in relation to social-perceptual and behavioral deficits which could contribute to the maintenance of loneliness over time. In doing so, the current study takes a novel 
approach, addressing potential mechanisms through which even transient loneliness may be consequential to individual health and well-being.

Transactional models of loneliness, which have been previously applied to chronic loneliness, posit that various symptoms or consequences of loneliness operate on the environment to exacerbate and maintain this negative-affective experience (Ernst \& Cacioppo, 1998). For example, these deficits could inhibit behaviors vital to the development of healthy interpersonal relationships. One such behavior is self-disclosure, which plays an important role in facilitating feelings of interpersonal closeness, intimacy, and connectedness (Collins \& Miller, 1994). Previous work has shown that loneliness is related to a lower willingness to disclose negative events (Rotenberg, 1997), as well as difficulty managing appropriate levels of disclosure during social interactions (disclosure flexibility; Chelune, Sultan, \& Williams, 1980). In line with transactional models of loneliness, evidence has suggested that this relationship between loneliness and disclosure behavior can be explained by poor social skills and negative perceptions of social interactions (Solano, Batten, \& Parish, 1982). Additionally, it is suspected that lonely persons may view self-disclosure as risky, as it makes these individuals vulnerable to potential social rejection (Rotenberg, 1997; Wittenberg \& Reis, 1986). Yet, whereas the avoidance of self-disclosure may be more comfortable for lonely persons in the shortterm, doing so prevents these individuals from reaping the social rewards and resources from disclosure interactions.

Early theorists within the communications and social psychology literature describe how disclosure of self-relevant information and meaningful events is necessary 
for individuals to perceive themselves as similar to others, and to know the needs and wants of those with whom they interact (Jourard, 1964). Additionally, much work has shown that self-disclosure is related to feelings of interpersonal closeness, trust, and liking (Collins \& Miller, 1994; Cozby, 1973; Wheeless \& Grotz, 1977). Thus, selfdisclosure is considered one of the "building blocks" of close, intimate relationships (Reis \& Shaver, 1988, p. 389). Ironically, lonely individuals are missing out these important social interactions, which could potentially aid in the development of close relationships and reduce loneliness over time.

\section{Capitalization}

Recent work has found that the disclosure of positive events, or capitalization, is a particularly positive interpersonal experience, which builds various personal and social resources (Gable, Reis, Impett, \& Asher, 2004; Gable, Gonzaga, \& Strachman, 2006; Langston, 1994). Specifically, sharing good news with others is associated with greater relationship satisfaction, intimacy, and trust, as well as reduced loneliness and increased life satisfaction, increased levels of positive affect above and beyond those experienced as a result of the event itself, and greater memorability of positive events (Gable et al., 2006; Gable \& Reis, 2010).

Interestingly, and as with negative event disclosure, the benefits of capitalization (e.g., trust, interpersonal closeness, reduced loneliness, positive affect) are contingent on the extent to which individuals perceive others as providing a supportive response to their positive-event disclosure (i.e., capitalization support; e.g., Reis, et al., 2010). Perceiving an enthusiastic, encouraging, caring, validating and understanding response (i.e., active 
constructive response) to a capitalization attempt is associated with increased positive affect and well-being, above and beyond the effects of the positive event (Gable \& Reis, 2010). Additionally, perceived responsiveness fosters positive attributions and prosocial behavior toward the response provider (Reis et al., 2010). Conversely, responses which point out the negatives of the event, or which disregard and ignore the positive event altogether (i.e., passive-destructive and active-destructive responses; Gable et al., 2004; Gable et al., 2006) are associated with greater loneliness, less satisfaction, less intimacy, and less trust. Importantly, perceived partner responsiveness is an integrative process, wherein one individual's perceptions of partner responsiveness influence not only his or her future disclosure and support-seeking behavior, but also the provision of supportive behavior to interaction partners (Clark \& Lemay, 2010).

Whereas much work has explored how loneliness influences engagement in negative event disclosure, less research has investigated the influence of this negativeaffective experience during the social sharing of positive events, or capitalization. Further, researchers have not examined how loneliness influences perceptions of responsiveness during positive-event disclosure. Examining loneliness in the context of the positive relationship process of capitalization would be important, as recent research and theory suggest that capitalization and perceived receipt of responsive behavior to capitalization attempts (i.e., capitalization support), is a stronger predictor of well-being and relationship functioning than the availability of support during difficult times (Gable et al., 2004; Gable et al., 2006). Further, previous research and theory within social psychology has provided evidence that positive and negative processes and behavioral 
systems are functionally independent. As much work has considered loneliness in the context of negative health behaviors or stress-related processes (e.g., negative-event disclosure), examining how loneliness influences engagement in positive behavioral and relationship processes, such as capitalization, would make an important contribution to the existing literature.

Additionally, as described above, no work to date has considered how transient experiences of loneliness relate to disclosure behavior, perceptions of responsiveness, and the provision of responsive behavior during disclosure interactions. Similar to deficits in negative-event disclosure, chronically lonely persons are also less likely to report disclosing positive events, given low levels of self-esteem and perceptual deficits in event importance (Arpin \& Mohr, in preparation). That lonely persons are less likely to engage in this positive interpersonal strategy is problematic, given the various positive social and personal benefits of sharing positive events and receiving a supportive response (e.g., reduced loneliness, increased interpersonal closeness, well-being, and self-esteem; Gable \& Reis, 2010). Whereas chronically lonely persons may be less likely to share positive events, these interactions are likely more common among individuals experiencing transient levels of loneliness. Moreover, current work does not consider how transient feelings of loneliness influence perceptual and behavioral deficits in the moment within positive social interactions (i.e., perceptions of capitalization support or responsiveness, and responsiveness to interaction partners' disclosure). In addition, though some work has shown that supportive capitalization interactions relate to lower levels of loneliness, research has not considered the impact of transient loneliness, experienced prior to a 
capitalization interaction, on perceptions and behaviors vital to the capitalization process. The present study addressed gaps in the literature by examining the effects of transient loneliness during the social sharing of positive events. Specifically, the study explored whether lonely feelings relate to more negative perceptions and less supportive behavior during a capitalization interaction.

\section{Loneliness and Responsiveness as Integrative, Interpersonal Processes}

Though previous work has shown that loneliness is related to deficits in social behavior, the study of loneliness has been primarily individual focused. That is, research examining the consequences of loneliness tends to focus on the perceptions and experiences of lonely individuals. Further, loneliness research which has considered the experience of nonlonely others interacting with or providing an evaluation of lonely persons takes a similar individual approach, as it typically manipulates perceptions of loneliness by having participants read a descriptive vignette about a 'lonely' individual. Such research has revealed that loneliness is a stigmatized experience, as nonlonely individuals often rate 'lonely' persons as less interpersonally attractive, less enjoyable interaction partners, and less desirable as future friends (Bell, 1985; Jones et al., 1982). Similarly, individuals who believe they are interacting with a lonely person exhibit less sociable behavior compared to when they are interacting with nonlonely others (Rotenberg, Gruman, \& Ariganello, 2002).

Despite this work, no research to date has examined perceptions and behaviors of lonely persons and their nonlonely interaction partners after an actual social interaction. This work would be important, as research and theory describe social interactions and 
interpersonal experiences as dyadic in nature, as they are shaped by the cognitions, needs, and motives of each individual in the interaction (e.g., Clark \& Lemay, 2010). Further, self-disclosure and perceived responsiveness have been described as transactional, integrative processes, wherein the experiences, perceptions, and behaviors of one individual influence the experiences, perceptions, and behaviors of the other, and vice versa (Clark \& Lemay, 2010; Reis \& Shaver, 1998). Lastly, the induction hypothesis of Cacioppo, Fowler, and Christakis (2009) describes how the behavioral consequences of loneliness (e.g., facial expressions, shyness, social-awkwardness) function to negatively influence individuals' experiences during social interactions with lonely others, consequently inducing loneliness in the nonlonely partner. Thus, in order to gain a full understanding of how loneliness functions in an interpersonal context, it would be important to examine the perceptions and behaviors of both transiently lonely individuals and their interaction partners. The current study took an interpersonal, dyadic approach by assessing the perspectives and experiences of lonely individuals and their nonlonely interaction partners following an actual positive-event disclosure interaction.

\section{Positivity Deficits in Capitalization Interactions}

Though the current loneliness literature has yet to examine whether transient experiences of loneliness influence the perceptions and behaviors of lonely persons and their interaction partners, recent work has considered how characteristics such as social anxiety and self-esteem (correlates of loneliness) influence individual and partner reports of responsiveness following the mutual disclosure of positive events. In particular, research by Kashdan, Ferssizidis, Farmer, Adams, and McKnight (2013) examined how 
positivity deficits related to social anxiety inhibit successful engagement in positive relationship processes, such as capitalization, which interferes with a socially anxious persons' ability to develop and maintain healthy, positive relationships. Kashdan's work showed that socially anxious individuals receive and provide less responsive behavior to their own and their partner's capitalization attempt. Additionally, interaction partners of socially anxious persons also reported receiving and providing a less supportive response during a positive event disclosure activity. Similar work has explored the influence of self-esteem on the perceived receipt of responsive behavior through individual and partner reports. As is the case with Kashdan et al.'s (2013) research on social anxiety, this research has shown that individuals with low self-esteem and partners of low self-esteem individuals perceive their partners as less responsive to the disclosure of positive events and benefit less from capitalization as a relationship repair process (MacGregor, Fitzsimons, \& Holmes, 2013; Smith \& Reis, 2012).

Despite the relation of loneliness to social anxiety and self-esteem, it is a distinct construct, the independent effects of which have not been examined in relation to the perceptions and provision of supportive behavior during capitalization interactions (Peplau \& Perlman, 1979). Based on known behavioral and perceptual deficits related to loneliness, and the known stigmatization of lonely persons by nonlonely others, it is possible that loneliness is related to similar positivity deficits within capitalization interactions as modeled in previous work on social anxiety and self-esteem. Building on this research, the current study examined the effects of loneliness on the perceived receipt and provision of responsive behavior, as well as social (i.e., partner liking and desire for 
friendship) and personal (i.e., positive affect) outcomes of capitalization, for both lonely persons and their interaction partners. In doing so, this study provides insight into one pathway through which transient levels of loneliness could perpetuate into chronic levels over time. Specifically, transient experiences of loneliness and related perceptual and behavioral deficits may interfere with the ability to effectively engage in and benefit from positive social interactions (e.g., capitalization interactions), making it difficult for individuals to overcome loneliness in the moment, and thus contributing to increasing levels over time.

Though it is clear that loneliness has widespread social and behavioral consequences, the majority of loneliness research to date is correlational and conducted through survey-based methods. Consequently, conclusions regarding the directional, causal influence of loneliness on social and behavioral deficits cannot be made. For instance, though loneliness is related to poor social skills and negative perceptions of social interactions, it is possible that these behaviors and cognitions lead to greater loneliness. To address these issues of directionality, research should investigate potential causal mechanisms through which loneliness influences subsequent social and behavioral outcomes through the use of experimental methods. Experimental laboratory methods are beneficial in establishing evidence of causal relationships among particular moods and behavioral outcomes, with the advantage of strong internal validity and experimental control (Wilson, Aronson, \& Carlsmith, 2010). Additionally, randomized experimental mood induction is beneficial in that it allows for the direct examination of how discrete moods relate to behavioral outcomes, controlling for the effects of pre-existing 
differences and baseline levels of mood (Westermann, Spies, Stahl, \& Hesse, 1996). Such work would be vital within loneliness research in order to isolate the intrapersonal and interpersonal consequences of transient loneliness, which may not only influence individuals' ability to develop interpersonal relationships, but also nonlonely others' desire to interact and maintain interpersonal contact with lonely persons.

\section{Current Investigation}

To address gaps in the literature, the current study utilized a loneliness-mood induction paradigm to explore the causal effects of transient feelings of loneliness on individual and interaction partner reports of perceived receipt and provision of supportive behavior during a capitalization interaction. As this was the first study to date to explore links among transient loneliness and subsequent perceptual and behavioral outcomes in a lab-based setting, participants were randomly paired into interaction dyads. While capitalization interactions most likely occur within established relationships (intimate or friendship), previous studies have utilized stranger dyads to investigate processes of capitalization and self-disclosure. For example, to clarify the interpersonal and intrapersonal benefits of capitalization interactions, Reis et al. (2010) randomly paired participants with confederates, with whom they engaged in a positive-event disclosure interaction. Similarly, Dindia, Fitzpatrick, and Kenny (2006) examined patterns of selfdisclosure among stranger dyads, finding few differences in lab-based disclosure behavior with romantic partners.

As such, the use of stranger dyads in lab-based disclosure interactions is a commonly accepted practice within the close relationships literature. Further, this 
allowed for a cleaner examination of how transient loneliness interferes with positive social interactions in the absence of preexisting perceptions and attitudes towards a relationship. In doing so, results of the current study provided insight into the interpersonal consequences of loneliness in the preliminary stages of relationship development (i.e., when individuals are first getting to know one another), which could be valuable in understanding how loneliness inhibits the development of interpersonal relationships and thus persists over time.

Thus, the current study investigated whether individuals receiving a loneliness induction (vs. a 'nonlonely' or social connectedness induction) reported higher levels of state loneliness, and thus perceive a 'nonlonely' interaction partner as less responsive to their disclosure of a positive event, provide less responsive behavior to their partner's positive event disclosure, and benefit less from the capitalization interaction (i.e., less positive affect, less enjoyment of the interaction, less liking of lab partner). In support of previously established multidimensional models of loneliness, the current study examined whether the receipt of a loneliness (vs. social connectedness) induction also related to lower levels of state self-esteem and perceptions of positive event importance. Further, as a test of the positivity-deficit perspective of loneliness, the study investigated the extent to which loneliness-related deficits in self-esteem, perceived event importance, and perceived responsiveness mediated the effects of the mood induction on outcomes of the event-sharing interaction (i.e., positive affect, enjoyment the interaction, and partner liking). 
Additionally, the investigation unpacked the extent to which 'nonlonely' (connectedness induction) interaction partners of 'lonely' participants experienced similar deficits in the perceived receipt and provision of responsiveness, as well as less positive outcomes of the capitalization interaction (i.e., less positive affect, lower liking towards lab partner). These effects were compared to those experienced by members of dyads wherein both individuals received a connectedness mood induction. Lastly, this study examined whether the effects of transient loneliness on perceptual and behavioral deficits among 'lonely' individuals and 'nonlonely' interaction partners differed based on chronic levels of loneliness.

Actor-focused hypotheses. Based on known social and perceptual deficits related to loneliness (i.e., negative perceptions of disclosure interactions; Rotenberg, 1997), it was predicted that individuals receiving a loneliness (vs. social connectedness) induction would report higher levels of state loneliness and lonely mood following a mood induction activity (H1.0). Previous literature has also demonstrated that lonely persons tend to report more negative perceptions of social interactions, lower levels of selfesteem, less positive attributions for successes, and are less responsive and more selffocused during social interactions (e.g., Bell, 1985; Wittenberg \& Reis, 1986). Further, previous work has demonstrated that lonely persons perceive positive events as less important compared to less lonely individuals (Arpin \& Mohr, in preparation). Thus, it was predicted that 'lonely' actors (relative to connected actors) would report receiving and providing less responsive behavior during the event disclosure activity (H1.1, H1.2), 
and report lower levels of self-esteem, and perceptions that events shared during the event-disclosure interactions were lower in importance (H1.3).

Based on Kashdan et al.'s (2013) positivity-deficit perspective and multidimensional models of loneliness (Ernst \& Cacioppo, 1998), it was predicted that 'lonely' actors (relative to their socially connected counterparts) would report lower levels of positive affect, less partner liking, and less enjoyment of the interaction (i.e., proxies for intrapersonal and interpersonal benefits of capitalization) following the eventsharing activity (H1.4). Further, it was anticipated that deficits in the perceived receipt and provision of responsiveness, as well as in the outcomes of the event-sharing activity (i.e., positive affect, partner liking, enjoyment of the interaction) among 'lonely' actors and 'connected' actors would be mediated by levels of state loneliness following the mood induction, revealing the mechanism through which the mood induction influences perceptions and behavior (Figure 4.0; H1.5).

In line with previous work demonstrating the importance of perceived responsiveness in predicting the intrapersonal and interpersonal outcomes of capitalization (Gable et al., 2004; Gable et al., 2006), it was expected that differences in levels of positive affect, partner liking, and enjoyment of the interaction (outcomes of the event-sharing activity) among 'lonely' actors and 'connected' actors would be mediated by perceptions of responsiveness (Figure 4.1; H1.6). More specifically, 'lonely' actors (compared to actors who receive a social connectedness induction) would report lower perceived responsiveness, and thus experience lower levels of positive affect, partner liking, and less enjoyment of the interaction follow the capitalization interaction. Lastly, 
based on multidimensional models of loneliness (Peplau \& Perlman, 1982), it was predicted that deficits in self-esteem and perceived event importance would mediate the effects of the induction on levels of positive affect, partner liking, and enjoyment of the interaction following the event-sharing activity (Figure 4.2; H1.7).

As the basis of the loneliness induction procedure included the recollection of previous experiences wherein participants felt a lack of belonging or isolation, experiences that are likely more salient for chronically lonely individuals, it was anticipated that the indirect effects of state loneliness on the induction-event-sharing outcome associations would be stronger for individuals at higher levels of chronic loneliness (Figure 4.3; H1.8). Similarly, the mediating effects of deficits in perceived responsiveness on the induction-event sharing outcome associations were predicted to be stronger for individuals at higher levels of chronic loneliness, who may consistently hold more negative perceptions of social interactions in general, and thus benefit less from the event-sharing activity compared to individuals at lower levels of loneliness (Figure 4.4; H1.9).

Interaction-partner hypotheses. Based on the induction hypothesis of loneliness (Cacioppo et al., 2009), as well as transactional models of social interactions and selfdisclosure (Reis \& Shaver, 1988), partners paired with 'lonely' actors (LONELY dyad condition), were anticipated to report receiving a less supportive response from their 'lonely' interaction partner, relative to partners interacting with 'connected' actors (CONNECTED dyad condition) (H2.1). Drawing from Clark and Lemay's (2010) integrative model of responsiveness, it was expected that partners paired with 'lonely' 
EXPERIMENTAL INDUCTION OF LONELINESS

actors (LONELY dyad condition), would report providing less responsive behavior to their 'lonely' interaction partner's positive-event disclosure (H2.2).

In line with Kashdan et al.'s (2013) work revealing the cross-over effects of positivity deficits within capitalization interactions, partners paired with 'lonely' actors (LONELY dyad condition) were expected to also report lower levels of positive affect, less liking toward their interaction partner (i.e., less liking towards the actor in the interaction), and less enjoyment of the interaction following the capitalization task (H2.3). Further, these differences in capitalization outcomes (i.e., positive affect, liking towards interaction partner, enjoyment of interaction) among partners paired with 'lonely' actors (LONELY dyad condition), and partners paired with 'connected' actors (CONNECTED dyad condition), would be explained by deficits in perceptions of responsiveness (Figure 4.5; H2.4).

\section{Pilot Study}

Prior to examining the effects of loneliness on the perceived receipt and provision of responsive behavior within lab-based event disclosure interactions, it was necessary to pilot the mood induction procedure. Experimental mood induction procedures were based on previous methods developed by Rotenberg and Flood (1998) and Cacioppo and Hawkley (2008), which employed the autobiographical recall method. The pilot study is only briefly summarized below, but a more detailed overview can be found in Appendix A.

Undergraduate psychology students ( $\mathrm{N}=68 ; 86 \%$ female) completed a brief 'selfreflection activity' through which they were prompted to either think back on a time 
when they felt like they were a member of a group (connectedness induction) or when they had no one to relate to (loneliness induction). Following induction activity participants rated how lonely they felt in the moment (0-Not at all, 4-Extremely) via an item on the negative affect subscale of the Positive and Negative Affect Schedule (Watson, Clark, \& Tellegen, 1998). Group differences in lonely mood following the induction activity were analyzed via an analysis of variance. Results indicated that induction conditions significantly differed in levels of lonely $\operatorname{mood}(F(1,61)=5.51, p=$ $\left..02, \eta^{2}=.08\right)$. Specifically, participants in the loneliness induction condition $(\mathrm{n}=33)$ reported significantly higher levels of lonely mood following the self-reflection exercise $(M=1.27, S D=1.04)$ compared to participants in the social connectedness induction condition ( $\mathrm{n}=30 ; M=.70, S D=.99)$.

Exploratory analyses revealed significant differences in general levels of negative affect between the two induction groups $\left(F(1,61)=4.28, p=.04, \eta^{2}=.06\right)$. As would be anticipated, participants receiving the loneliness induction reported higher levels of negative $\operatorname{mood}(M=9.94, S D=2.47)$, relative to participants who received the connectedness mood induction $(M=8.52, S D=2.93)$. To determine whether the mood induction influenced levels of loneliness, above and beyond increases in negative affect, an analysis covariance was conducted, controlling for levels of general negative affect. Results revealed marginally significant differences in lonely mood between induction groups, when controlling for general negative affect $\left(F(1,60)=3.01, p=.09, \eta^{2}=.05\right){ }^{1}$

Results of a 2 X2 factorial ANOVA revealed no gender differences in lonely mood following the induction $(F(1,61)=.82, p=.37)$. Additionally, there were no differences 
in the effects of the induction on levels of lonely mood among men and women $(F(1,61)$ $=1.65, p=.20)$. Despite the absence of gender differences in induction effects as demonstrated in the pilot study, it was determined necessary to fix gender in the primary study. As the primary study included an in-lab interaction among two participants, it was anticipated that focusing on same gender dyads would allow for greater experimental control, particularly in regards to gender differences in communication-style, selfdisclosure behavior, and social support provision (e.g., Dindia \& Allen, 1992).

\section{Primary Study Method}

\section{Participants}

Six-hundred and forty-eight undergraduate psychology and communication students (72\% female) were recruited from Portland State University via in-class announcements for participation in an online survey for extra credit. At the end of the online survey, participants who did not exhibit risk of depression, who were over the age of 18 , female, and who did not participate in the pilot study were provided with an advertisement for a second research participant opportunity, which involved participation in a 'separate' lab study for additional extra credit and entrance into a drawing to win one of thirty $\$ 25$ gift cards. Out of the participants who were eligible and interested in participating in the lab study $(\mathrm{N}=368), 268$ signed up for a lab session (134 interaction dyads). Sixty-four percent of possible full lab sessions were attended. Data from three lab sessions was eliminated during data cleaning, as participants indicated that they were close friends prior to the lab session (one pair of participants were siblings), thus rendering a sample of $\mathrm{N}=166$ (83 interaction dyads). Two individuals in our final 
sample were excluded from key analyses, as they correctly guessed the purpose of the study during debriefing (i.e., loneliness and social interactions), thus rendering a final sample size of $\mathrm{N}=164$.

Average age for the final sample used in the lab study was 25.09 ( $S D=8.62$, range $=18-64)$. Seventy-percent of the lab participants were Non-Hispanic/White, $14 \%$ Hispanic/Latino, 10\% Asian, 5\% Black American, 5\% American Indian or Alaskan, 2\% Hawaiian/Pacific Islander, 7\% Other. Eighty-one percent identified as Heterosexual, 12\% Bisexual, 3\% Homosexual, and 4\% Non-binary. Seventy percent of participants were in a romantic relationship. Fifteen percent indicated having a high school diploma, 29\% an associate degree, $48 \%$ some college education but no degree, $6 \%$ a bachelor's degree, and $1 \%$ had participated in or completed graduate studies. Fifty-nine percent of participants were employed, and 97\% percent indicated earning less than $\$ 44,000$ per year.

\section{Initial Online Survey Procedure}

Recruitment materials included a link to a brief online survey (10 minutes), which assessed basic demographics, chronic loneliness, perceived availability of support, depression, social anxiety, and self-esteem. Participants were offered extra credit for completion of this online survey. The online survey functioned as an initial assessment, as well as a screening tool to identify individuals at risk for depression, under the age of 18 , and who participated in the pilot study (eligibility criteria). At the end of the online survey, eligible participants received an email advertisement describing an opportunity to participant in a second, seemingly separate research study. 
Eligible participants interested in participating in the lab study were provided a link to an online lab session scheduler (YouCanBook.me) where they could sign up for a 30-40 minute lab time. The online scheduler was programmed so that participants could not sign up for a lab time within a week of taking the initial online survey. Participants automatically received an email reminder at 5:00 pm the day before their session, as well as an optional text message reminder two hours prior to their lab session. Two participants were allowed to sing-up for each lab time. Participants were randomly paired in lab sessions based on their selected lab time.

\section{Laboratory Session Procedure}

All lab sessions were held in a communication research lab. The lab space included a lobby for participant sign-ins and completion of informed consent, an interaction room, and a separate computer room. The interaction room contained two comfortable chairs facing one another, as well as a video camera mounted on the wall above the door to the room entrance. The computer lab contained six rows of computers facing a whiteboard. A small flip-cam was set up adjacent to the participant's computer.

Prior to their scheduled lab session, participants were randomly assigned into LONELY (loneliness induction, connectedness induction) or CONNECTED (connectedness induction, connectedness induction) dyads. As two participants attended each lab session, participants were randomized first to dyad-level conditions. Individuals within LONELY (loneliness, connectedness) dyads were then randomly assigned to the loneliness versus connectedness mood induction conditions. Individuals within LONELY dyads, and assigned to receive the loneliness mood induction were also assigned the role 
'Participant A'. Participants in CONNECTED dyads were both assigned to receive the connectedness mood induction, though one participant in each CONNECTED dyad was assigned the 'Participant A' title. As will be described below, Participant A always stayed in the interaction room for the self-reflection exercise (mood induction), and Participant A was always asked to go first in the event sharing activity. The Participant A versus lab partner distinction within CONNECTED dyads enabled research assistants to remain blind to dyad-level and individual-level conditions in each lab session.

Upon arriving at the lab, participants were administered informed consent. Participants who did not consent to being video-taped were asked if they would be comfortable being audio-recorded. After both participants arrived and completed informed consent, a research assistant led them into the interaction room to begin the study. ${ }^{2}$ After both participants were seated in the interaction room, the research assistant began describing the study as an investigation of how people think about and discuss their experiences with other people. Participants were informed that they would be completing three brief activities ('Getting-to-Know-You', 'Self-Reflection Activity', 'Event-Sharing Activity') and two brief surveys (manipulation check, post-experiment survey) throughout the lab session. Participants completed some of the activities together and some alone. These activities and the general lab procedure are outlined in Appendix B (Figures 4.6 and 4.7). Below is a more detailed description of each part of the lab procedure.

Getting-to-know-you activity. The first activity was a 5-minute exercise during which participants switched back and forth asking one another casual getting-to-know- 
you questions. This exercise acclimated participants to the lab setting, the video cameras, and to their lab partner. Additionally, it will prepare participants for the third part of the lab session, wherein they will be asked to take turns disclosing positive events to one another. Example questions included "What's your name?", "What's your year in school?", and "What's your favorite color?". The research assistant gave the following instructions and then left the room for the duration of the activity:

For this part of the study, we want you to spend a bit of time getting to know each other. Please spend the next five minutes switching back and forth between these questions, which will help you get to know one another. I will set a timer, start the camera, and leave the room. I will reenter after five minutes and let you know when time is up (Appendix B).

Self-reflection exercise: Experimental mood induction. Following the gettingto-know you activity, participants were separated for a 5-minute self-reflection exercise (experimental mood induction). Participant A was always asked to complete this exercise in the interaction room. The other participant was led into the computer room by a research assistant, where she was administered the same exercise.

Experimental induction procedure. The mood induction methods utilized in the self-reflection exercise were identical to those utilized in the pilot study. However, it should be noted that participants in LONELY dyads received different conditions. That is, Participant A received a loneliness induction, while her lab partner received the connectedness induction. Induction procedures were based on methods developed by Rotenberg and Flood (1998) and Cacioppo and Hawkley (2006), which utilize the 
autobiographical recall method. In both 'loneliness' and 'connected' conditions, participants were given a small envelope, each containing two slips of paper with a different statement asking participants to think back on a specific type of life experience. The statements within each envelope differed depending on the induction condition.

Specifically, participants assigned to receive the 'loneliness' induction were given an envelope containing two items adapted from the UCLA loneliness scale. Specific statements included: "Think of a time when you felt you lacked companionship. Perhaps you felt like you had no friends" and "Think of a time when you were no longer close to anyone. Perhaps you felt like you just didn't belong." It is important to note that neither of these items contained the word loneliness, nor were participants directly asked to recall a time when they felt lonely. Participants receiving the 'connectedness' induction were provided two items adapted from methods used by Cacioppo and Hawkley (2006). Specific statements included: "Think of a time when you felt a sense of belonging. Perhaps you were a member of a group" and "Think of a time when you felt you had someone you could share anything with. Perhaps this was a person who was or who could be your best friend."

Participants were guided through the 'self-reflection exercise' (mood induction) with a computerized interval timer (Intervals 1 and $2=1.5$ minutes; Interval $3=2$ minutes). An alarm sounded after the end of each time interval. In both loneliness and connectedness mood conditions, participants were told to spend the first two intervals (each 1.5 minutes) taking out one slip of paper at a time, reading it to themselves, and thinking back on a time when they really experienced what was described on each slip of 
paper. For the third interval, participants were asked to describe these experiences in detail out loud to the video camera. Research assistants started the timer, and left the room for the duration of the activity ( 5 minutes). Participants received the following instructions for the induction activity:

For this part of the study, you will be completing a brief self-reflection exercise. You will be guided through this exercise using the contents of this envelope and this interval timer (point to the interval timer on the laptop). In this envelope are two slips of paper. Each slip of paper contains a statement asking you to think back on a specific type of life experience. As you can see, there are three intervals on the timer. For the first two intervals, I ask that you take each slip of paper one at a time, read it to yourself, and spend time thinking back on a time when you really felt what is described on each slip of paper. For the third interval, start describing these experiences out loud for two minutes. A final alarm will alert you the end of the activity (Appendix B).

Manipulation check. At the end of the five-minutes, a research assistant reentered participants' rooms and administered a brief survey. The survey included items assessing levels of state loneliness, state social connectedness, and state self-esteem (specific measures provided below). Participants were instructed to alert the research assistants when they were finished taking the survey. If one participant finished before the lab partner, they were instructed to wait in their assigned for the other participant to complete the exercise. 
EXPERIMENTAL INDUCTION OF LONELINESS

Event-sharing activity: Capitalization interaction. Upon completion of the self-reflection exercise and manipulation check survey, both participants were led back into the interaction room for an event-sharing activity. This activity was modeled after Gable et al.'s (2006) capitalization lab paradigm, wherein interaction partners take turns discussing positive events (Appendix B). As noted above, the research assistant always instructed Participant A to share his/her event first. Participants were told that the purpose of this activity was to explore how individuals talk about recent events with other people. A research assistant provided the following instructions:

For this activity, I would like for each to spend time discussing a recent positive event with one another. You are free to choose any event that comes to mind. It may be something that happened to you recently or in the past that continues to make you happy, something going on now, or something you anticipate will happen in the future. This event can be anything good (big or small) that has either happened recently or that you anticipate happening in the future. However, it must be something that has been on your mind lately. You will each have five minutes to describe your events to one another (point to interval timer). When you are describing your event, try to go into as much detail as possible. When hearing about your partner's event you are free to talk as much or as little as you wish. In both situations, try to engage in a conversation that is as close as possible to a normal interaction you may have with a friend or someone you know. I will come in at the end of the ten minutes for the 
final part of the study. Any questions? Okay, [Point to Participant A], why don't you start by sharing your event first (Appendix B).

Post-experiment questionnaire. Following the event-sharing task, participants were separated again for the post-experiment survey (Appendix B). Once again, Participant A was administered the survey in the interaction room, and the other participant was administered the survey in the computer room. As will be described in more detail below, the post-experiment survey assessed participants' perceptions of partner responsiveness and self-reported provision of responsive behavior during the capitalization interaction task, as well as current levels of positive affect and loneliness, liking towards interaction partner, and enjoyment of the interaction. At the end of the survey, participants were provided with a link to an online form where they provided their course information to redeem their extra credit. Participants were also entered into the gift card lottery following their participation in the lab session.

Debriefing. Following completion of the post-experiment survey, participants were fully debriefed and probed for suspicion (funnel debriefing procedure, Appendix B; Bargh \& Chartrand, 2000). Additionally, to ensure that the effects of the induction and disclosure of the use of deception in the study did not have long-lasting negative effects, participants were asked to complete a brief self-affirmation activity. For the selfaffirmation activity, participants spent 1-2 minutes writing down the most positive aspects on themselves on a $3 \mathrm{X} 5$ notecard. Participants were asked to take their selfaffirmation card home with them. Upon the completion of the self-affirmation activity, 
participants were provided with a list of contact information and free student mental health resources.

\section{Measures}

Online survey measures. As described above, participants were first recruited for an online survey of 'student relationships and attitudes'. Though the online survey and lab study were advertised as separate research studies, participant responses to the online survey were linked to participants' lab data. Online survey data was used as a randomization check and to test for individual differences in perceptions and behaviors during the lab study (Hypotheses 1.8-1.9). Additionally, initial assessment data on age, participation in the pilot study, and depression were used in screening procedures for the lab study.

Basic demographics. Participants responded to questions probing basic demographic information (e.g., age, gender, ethnicity), and indicated whether or not they had participated in the pilot study ('Study of Self-Reflection and Social Perception'). As described above, participants who indicated being under the age of 18 and participating in the pilot study did not receive the advertisement for the lab study.

Chronic loneliness. Chronic levels of loneliness were assessed via the UCLA Loneliness Scale (Russell, 1996). Participants responded to twenty statements relating to general feelings of belongingness, using a 4-point rating scale (1-Never, 4-Always). Example items included "How often do you feel that you are 'in tune' with the people around you?" and "How often to you feel that your relationships with others are not meaningful?". Items demonstrated adequate internal consistency (Cronbach's alpha = 
.86 ), consistent with previous research using this measure (Cronbach's alpha $=.86-.94$, Pressman et al., 2005; Russell, 1996).

Depression. Risk of depression was assessed via screening procedures similar to those administered by Mohr et al. (2013). Specifically, participants responded to a series of six items assessing the occurrence of primary symptoms of depression over the past two weeks (0-No, 1-Yes). Items were based on diagnostic criteria for depressed mood, as delineated in the Diagnostic and Statistical Manual of Mental Disorders (Diagnostic and Statistical Manual of Mental Disorders V [DSM-V]; American Psychiatric Association, 2000). Example items included "Have you experienced depressed mood nearly every day for the past two weeks?" and "Have you experienced loss of interested over the past two weeks?". Participants who indicated experiencing depressed mood and/or loss of interest and pleasure in life activities, and at least five additional symptoms which cause clinical significant daily impairment in social, work, and other important areas of functioning, were flagged for risk of depression. At risk individuals were immediately emailed a list of free mental health resources. Additionally, these individuals were not provided with the advertisement for the lab portion of the study.

In addition to the screening procedures described above, the Short DepressionHappiness Scale was included as a more continuous measure of depression (Joseph \& Lewis, 1998). As depression is a strong correlate of loneliness, this measure was used in a randomization check, and as a control for analyses assessing individual differences in chronic levels of loneliness (H1.8-H1.9). Participants responded to a series of six items probing feelings of dissatisfaction, cheerlessness, and meaninglessness over the past 7- 
days, using a 4-point rating scale (0-Never, 3-Often). Example items included "I felt dissatisfied with my life," "I felt pleased with the way I am," "I felt that life was enjoyable." Positively-framed items were reverse scored and aggregated with negativelyframed items to create a composite depression score (Joseph, Linley, Harwood, Lewis, \& McCollam, 2004). Items demonstrated adequate internal consistency (Cronbach's alpha = .84 ), in line with previous studies using this measure (Cronbach's alpha $=.86$; Joseph et al., 2004).

Manipulation Check. Following the completion of the 'self-reflection activity', participants completed a brief manipulation check survey, which assessed state levels of loneliness and lonely mood, state social connectedness, and state self-esteem.

State loneliness. State levels of loneliness were assessed via a validated two-item assessment of loneliness to assess state levels of loneliness after the induction procedure. Participants used a 7-point Likert scale to rate the extent to which they felt lonely ("I am feeling lonely right now," “At this moment, I feel quite lonely”; 1- Strongly disagree, 7Strongly agree; Zhou, Sedikides, Wildschut, \& Gao, 2008). As suggested by Zhou et al. (2008), responses on each of these two items were combined to form a single state loneliness score (Cronbach's alpha $=.93)$.

Participants also rated the extent to which they feel lonely mood "now or in the past few minutes," using a 5-point rating scale (1-Very slightly or not at all, 5Extremely), via an item on the negative affect subscale on the Positive and Negative Affect Schedule (Watson et al., 1988). This item was included to compare to scores on the two-item state loneliness measure, and to provide a comparison to the mood induction 
effects observed in the pilot study. General levels of negative affect, which were adjusted for in manipulation check analyses, were also computed using the PANAS. Negative affect items (e.g., distressed, guilty, ashamed) were summed to create a negative affect score. Items demonstrated adequate internal consistency (Cronbach's alpha $=.84)$, consistent with previous studies using this measure as an assessment of momentary negative affect in a college student sample (Cronbach's alpha $=.85$, Watson et al., 1988).

State social connectedness. Feelings of social connectedness following the induction activity were assessed via two-items developed by Wildschut, Sedikides, Routledge, Arndt, and Cordaro (2010). The items were "Thinking about this past experience made me feel loved" and "...feel connected to loved ones." Participants rated these items on a 7-point scale (1-Strongly disagree, 7-Strongly agree). Responses were summed to create a composite state social connectedness score. The items demonstrated high internal consistency (Cronbach's alpha $=.88)$, in line with previous work using this measure as an assessment of state social connectedness (Cronbach's alpha $=.90$; Wildschut et al., 2010).

State self-esteem. Levels of self-esteem following the mood induction were assessed via the social and performance sub-scales of the State Self-Esteem Scale (Heatherton \& Polivy, 1991). Participants rated the extent to which a series of items were true for them "right now, in the moment," using a 5-point Likert scale (1-Not at all, 5Extremely). Example items included "I feel confident about my abilities," "I feel confident that I understand things," and "I feel self-conscious." Responses on items from each subscale were summed to create a performance self-esteem and social state self- 
esteem score. Items demonstrated adequate reliability (Cronbach's alpha $=.90$ ), consistent with previous uses of this measure (Cronbach's alpha $=.86-.92$; e.g., Heatherton \& Polivy, 1991; Ikegami, 2002).

Post-interaction measures. Following the event-sharing interaction, participants were again separated for a brief survey, which assessed current levels of positive affect and perceptions of their own and their partners' behavior during the event-sharing activity.

Positive affect. Post-interaction levels of positive affect were assessed via the PANAS (Watson et al., 1988). As in the manipulation check survey (post-induction), participants were asked to rate the extent to which they felt a series of positive and negative moods "now or in the past few minutes," using a 5-point rating scale (1-Very slightly or not at all, 5-Extremely). General positive affect was computed by summing the positive affect items (e.g., interested, enthusiastic, excited) and the negative affect items (e.g., sad, anxious, disappointed). Items on the positive affect sub-scale (Cronbach's alpha $=.91)$ demonstrated adequate internal consistency, consistent with previous work using this measure as an assessment of momentary affect in college student samples (positive affect, Cronbach's alpha $=.88$; Watson et al., 1988).

Perceived event importance. Following the event-sharing activity, participants were asked to rate the perceived importance of their own and their partner's shared positive events. Items were adapted from those developed by Kashdan et al. (2013) in a study of social anxiety and romantic partner capitalization. The first item assessed the participant's perceived importance of their lab partner's positive event ("How important 
was the event your lab partner shared with you?'). The second item assessed participants' perceived importance of their own positive event which they shared with their lab partner during the event-sharing activity. Participants rated each item on a 5-point rating scale (1Not at all important; 5-Extremely important). Analyses in the current study utilized only the first item, which assessed participant perceptions of importance for the event which they shared with their lab partner during the event sharing activity. Previous capitalization research has utilized a single item to reliably assess perceived event importance within lab-based capitalization interactions (e.g., Gable et al., 2006).

Perceived responsiveness. Perceived receipt of a supportive response during the event-sharing interaction was assessed via the 4-item Perceived Responses to Capitalization Attempts Scale (PRCA; Gable et al., 2006), which assesses four validated responses to capitalization attempts along two dimensions (i.e., active versus passive; constructive versus destructive). Specifically, this scale assesses the perceived receipt of active-constructive, passive-constructive, active-destructive, and passive-destructive responses to capitalization. Participants rated the extent to which the four items applied to their lab partner's response to their positive-event disclosure on a 5-point rating scale (1Not at all true of our interaction; 5-Very much true of our interaction). Example items included "When I told my lab partner about my positive event, he/she reacted enthusiastically to my good event," “...pointed out the potential problems or down sides of the good event," “...said little, but I knew he/she was happy for me," “...seemed disinterested.” As suggested by Gable et al. (2004), a composite responsiveness score was computed by subtracting the average of the latter three items from the first item, with 
higher scores indicating a more positive response. Each item in the 4-item version of the Perceived Responses to Capitalization Attempts Scale (PRCA; Gable et al., 2006) represents a distinct category of responsiveness (i.e., active-constructive, passiveconstructive, active-destructive, and passive-destructive), only two of which were correlated in this sample ("Reacted enthusiastically to this event" and "Seemed disinterested."; $r=-.46, p<.001)$. Thus, given conventions delineated by Bollen and Lennox (1991) regarding indicators as causes (versus effects) of a latent construct, it was not appropriate to test for internal consistency of these four items. ${ }^{3}$

Perceived provision of responsive behavior. Self-reported provision of responsive behavior during the event-sharing activity was computed via 10-items developed by Kashdan et al. (2013). These items were originally adapted from the Reis' (2003) Responsiveness Scale, though wording was changed to apply to participants' own responses to an interaction partner's disclosure of positive events. Using a 5-point rating scale (1-Not at all, 5-Very much), participants rated the extent to which the 10-items applied to their behavior in response to their lab partner's positive event disclosure during the event-sharing activity. Example items included "I was responsive to my lab partner's needs," "I really listened to my lab partner," "and I expressed liking and encouragement toward my lab partner." As with the original version of this measure (Reis, 2003), items were averaged to create a mean score for provided responsiveness. Previous work has utilized this measure as an assessment of perceived responsiveness, finding adequate reliability and validity (e.g., Cronbach's alpha $=.89-.94$; Gable et al., 2006). Though previous research using the adapted version of this measure (self-reported provision of 
responsive behavior; Kashdan et al., 2013) does not report reliability information, the items showed adequate internal consistency (Cronbach's alpha $=.88$ ), comparable to the original version of this measure.

Enjoyment of the interaction. Liking towards interaction partner and enjoyment/amusement of the interaction were assessed via 8-items developed by Reis et al. (2010). Participants rated the extent to which they enjoyed the interaction on a 9-point rating scale (1-Strongly disagree, 9-Strongly Agree). Example items include statements such as "I enjoyed my interaction with the other participant," "This interaction was a lot of fun," "Interacting with the other participant was entertaining and amusing" (Reis et al., 2010). Items were combined to create a single enjoyment/amusement score. Items demonstrated high internal validity (Cronbach's alpha $=.91)$ consistent with previous uses of this measure (Cronbach's alpha $=.92-.93$; Reis et al., 2010).

Liking towards lab partner. Partner liking was similarly assessed with 4-items which participants rated on 9-point Likert scale (1-Strongly disagree, 9-Strongly Agree). Items include "I liked the other participant," "I would like to interact with the other participant again," "The other participant is someone I could see having as a friend," and "The other participant was warm." Items were combined to create a single liking score. Items on both scales demonstrated adequate internal consistency (Cronbach's alpha $=$ .89 ), consistent with previous uses of this measure (Cronbach's alpha .87-.92; Reis et al., 2010).

Pre-study familiarity with lab partner. At the end of the post-interaction survey, participants indicated the extent to which they previously knew or had interacted with 
their lab partner, prior to the lab interaction. Specifically, participants responded to a single item, "How well did you know your lab partner before participating in the study today?" using a 3-point rating scale (0-Not at all, never interacted before, 1- Somewhat, have talked to this person once or twice in a class, 2-Very well, interacted on a regular basis and talk to this person frequently). Previous research examining lab-based interactions among stranger dyads has incorporated similar items to reliably assess familiarity among lab partners prior to participation in the study (e.g., Reis, Maniaci, Caprariello, Eastwick, \& Finkel, 2011).

\section{Data Analysis Strategy}

Data screening. Prior to hypothesis testing, data were examined for outliers and for distributional assumptions. As suggested by Mills and D’Mello (2014), outliers on outcome variables were identified via standardized z-scores. Cases for which the absolute value of the z-score was greater than 2.5 were not included in analyses (no cases were flagged as outliers on the lonely mood and state loneliness scores. Three outliers were flagged on the enjoyment of the interaction measure). Normality was assessed for key study variables via the examination of histogram plots.

All study variables met distributional assumptions for normality, with the exception of state loneliness, perceived responsiveness, and post-interaction enjoyment and interaction partner liking. Distributions for responsiveness, enjoyment, and liking exhibited slight negative skew, and state loneliness demonstrated slight positive skew. Square root data transformations were utilized on these three variables, and normality was improved for each variable. Interestingly, preliminary exploration of group 
differences did not differ when raw versus transformed variables were used. Additionally, as will be described below, bootstrapping was used in key study analyses, which addresses concerns with violations to distributional assumptions (Howell, 2010). Thus, concerns with slight deviation in normality for enjoyment, liking, state loneliness variables was attenuated, and raw variables were retained in all analyses for ease of interpretation.

Test of nonindependence. Typical research incorporating participant dyads considers the application of dyadic-analytic methods to account for interdependence of data for individuals within dyads. As participants in this study were randomly paired in dyads, a test of non-independence was conducted to examine whether dyadic data methods should be used for analyses. Pairwise partial correlation coefficients were computed between dyad members' scores on key outcome variables of interest (i.e., postinteraction loneliness and positive affect, enjoyment of the interaction, partner liking, perceived responsiveness, and provision of responsive behavior). Pairwise partial correlations controlled for key independent variables (i.e., individual-level condition, dyad-level condition, post-induction loneliness). As suggested by Kenny, Kashy, and Cook (2006), a liberal significance-level was used $(p=.20)$, to reduce power limitations characteristic of tests for nonindependence. As expected, the data did not exhibit significant nonindependence on key study variables. Specifically, dyad members' postinteraction levels of positive affect $\left(r_{p}=.15, p=.22\right)$, perceived responsiveness $\left(r_{p}=\right.$ $.002, p=.98)$, provision of responsiveness $\left(r_{p}=-.12, p=.33\right)$, enjoyment $\left(r_{p}=.05, p=\right.$ $.68)$, and partner liking $\left(r_{p}=-.07, p=.57\right)$ were not significantly related. 
Thus, the most appropriate data analytic strategy was to analyze 'actor' and 'partner' data separately and at the individual-level (rather than dyadic-level) of analysis. A power analysis revealed that the final sample size $(\mathrm{N}=164)$ would provide sufficient statistical power for individual-level analyses. Specifically, with a medium effect size of the induction effect, such as that observed in the pilot study $\left(\eta^{2}=.08\right)$, results of a power analyses revealed the need for a minimum of 52 participants per individual-level condition.

As previously described, participants were paired into random interaction dyads. Thus, it was determined that the study would require at least 78 dyads (52 dyads made up of a 'lonely' and 'connected' participant, 26 dyads made up of two 'connected' participants) to have sufficient power to test for between-groups differences. Within these dyads, individual-level conditions would include participants receiving a loneliness induction (52). Participants who received a connectedness induction and interacting with someone who received a loneliness induction (52), and participants who received a connectedness induction and interacting with a participant receiving a connectedness induction (52). Based on criteria provided by Fritz and MacKinnon (2007), it was determined that this sample size would allow for sufficient power to test for indirect effects using the bias-corrected bootstrap method, with medium effects of the independent variable on mediator, and mediator on dependent variable (suggested sample size, $N=115-148)$. Our final sample size $(\mathrm{N}=164)$ exceeded the target sample size (156), as determined by preliminary power analyses. 
Accordingly, hypotheses were tested via a series of analyses of variance (randomization check and manipulation check) and multiple regression analyses via SPSS data analytic software (IBM Corp, 2013). Bias-corrected confidence intervals were estimated for indirect effects via Preacher and Hayes (2007) INDIRECT macro, and moderated mediation was estimated via Preacher and Hayes (2007) MODMED macro. The estimation of indirect effects via bootstrapping is suggested when testing mediation in experimental research with small to moderate sample sizes (Shrout \& Bolger, 2002). Additionally, the estimation of bias-corrected confidence intervals via bootstrapping provided for a more powerful test of mediation than traditional methods (e.g., Sobel test), without the strict assumptions of normality for the sampling distributions (Hayes, 2009).

\section{Results}

\section{Descriptives}

On average, participants reported moderate levels of chronic loneliness $(M=$ $33.33, S D=9.22$, range $=15-62$ ). Average levels of depression in this sample, as assessed via the Depression-Happiness Scale, were low $(M=4.63, S D=3.30$, range $=0$ 13), which was expected given eligibility criteria for the lab study (screened out individuals at risk for depression). Across induction conditions, participants reported an average of 1.84 for lonely mood $(S D=1.15$, range $=1-5)$, and 7.17 for state loneliness $(S D=3.04$, range $=4-14)$ following the induction activity. Mean ratings of event importance were high, for both the participant's own event $(M=4.28, S D=.92$, range = $1-5)$, and for participant's perceived importance of their lab partner's event $(M=4.53, S D$ $=.78$, range $=2-5)$. Additionally, mean levels of state self-esteem were relatively high in 
this sample $(M=27.14, S D=4.80$, range $=10-35)$. Average received responsiveness during the event-sharing interaction was $2.34(S D=1.13$, range $=-.67-4.00)$. On average, participants reported providing a supportive response to their lab partner's event disclosure during the event-sharing activity $(M=4.23, S D=.54$, range $=2.60-5.00)$. Mean positive affect following the event-sharing interaction was $31.79(S D=9.07$, range $=10-50)$.

In line with previous literature and key study hypotheses, participants at higher levels of chronic loneliness reported greater levels of depression $(r=.68, p<.001)$. Chronic loneliness was also associated with greater state loneliness following the mood induction $(r=.48, p<.01)$; lower state self-esteem $(r=-.49, p<.01)$, less received responsiveness $(r=-.18, p<.05)$, less provided responsiveness $(r=-.16, p<.05)$, and less positive affect after the event-sharing activity $(r=-.24, p<.01)$. Additionally, state loneliness was related to great lonely mood $(r=.70, p<.01)$, lower state self-esteem $(r=$ $-.52, p<.01)$, less received responsiveness $(r=-.16, p<.10)$, less provided responsiveness to lab partner event-disclosure $(r=-.16, \mathrm{p}<.10)$, less enjoyment of the interaction $(r=-.20, p<.01)$, lower liking of the interaction partner $(r-.17, p<.05)$, and less positive affect following the event-sharing interaction $(r=-.27, p<.01)$. Bivariate correlations and descriptive statistics for key study variables are presented in Table 3.0.

\section{Randomization Check}

Between-groups comparisons were made to assess the effectiveness of randomization into dyad-level (LONELY versus CONNECTED dyads) and individuallevel conditions (loneliness induction versus connectedness induction conditions). A 
EXPERIMENTAL INDUCTION OF LONELINESS

series of one-way ANOVAs were conducted to compare background variables for individuals assigned to LONELY dyads $(\mathrm{n}=111)$ versus CONNECTED dyads $(\mathrm{n}=53)$, and for individuals randomized into loneliness $(\mathrm{n}=54)$ versus connectedness induction conditions within LONELY dyads $(\mathrm{n}=110)$. Groups were compared on baseline levels of loneliness, depression, and age. Comparisons of individuals randomized into dyadlevel conditions (LONELY versus CONNECTED dyads) revealed that groups did not differ on baseline chronic loneliness $(F(1,162)=.00, p=.95)$, depression $(F(1,162)=$ $.55, p=.46)$, or age $(F(1,162)=.03, p=.86)$. Similarly, individuals assigned to loneliness versus connectedness individual-level conditions (within LONELY dyads) did not differ in baseline levels of loneliness $(F(1,162)=.63, p=.43)$, depression $(F(1,162)$ $=1.02, p=.31)$, or age $(F(1,162)=.00, p=.96)$.

\section{Manipulation Check}

An analysis of variance was conducted to compare state levels of loneliness and lonely mood among individuals who received the loneliness $(\mathrm{n}=54)$ versus social connectedness $(\mathrm{n}=111)$ induction. Results of the manipulation check were in line with Hypothesis 1.0, revealing significant group differences on both the two-item measure of state loneliness $\left(F(1,161)=7.55, p=.007, \eta^{2}=.05\right)$, and the loneliness mood item from the PANAS $\left(F(1,161)=5.91 . p=.02, \eta^{2}=.04\right)$. Specifically, participants in the loneliness condition reported significantly higher levels of lonely mood $(M=2.15, S D=$ $1.22)$, and state loneliness $(M=8.09, S D=3.02)$, relative to individuals who received a social connectedness induction (lonely mood, $M=1.69, S D=1.09$; state loneliness, $M=$ $6.73, S D=2.95)$. There were also significant group differences in state social 
connectedness $\left(F(1,161)=35.99, p<.001, \eta^{2}=.18\right)$, such those who received a social connectedness induction reported significantly higher levels of state social connectedness $(M=7.87, S D=1.98)$ than participants who received the loneliness induction $(M=5.77$, $S D=2.33)$.

Induction conditions were also compared on post-induction reports of negative affect. As loneliness is a form of negative affect, it was important to establish that loneliness and connectedness actor induction groups differed in lonely mood, unique from general levels of negative affect. There were marginally significant group differences in negative affect following the induction $\left(F(1,161)=3.03, p=.08, \eta^{2}=.02\right)$. Additionally, an analysis of covariance was conducted to examine the effects of the mood induction on lonely mood and state loneliness, adjusting for post-induction negative affect. Induction effects remained significant for state loneliness, when controlling for negative affect $\left(F(1,160)=4.47, p=.03 ; \eta^{2}=.03\right)$. Induction effects on lonely mood, as assessed by the PANAS, were marginal when controlling for negative affect $(F(1,160)=$ $\left.2.93, p=.08, \eta^{2}=.02\right)$.

Lastly, as an exploratory step, a moderated regression analysis was conducted to examine whether the effects of the induction on state loneliness and lonely mood differed based on grand mean-centered chronic loneliness, as assessed in the initial online survey. Chronic loneliness significantly related to post-induction levels of state loneliness $(b=$ $.15, p<.001)$, and lonely mood $(b=.06, p<.001)$. However, interaction effects of chronic loneliness and induction condition were not significant for state loneliness $(b=$ 
$.07, p=.15)$, or lonely mood $(b=.02, p=.19)$. Thus, the effect of the induction on state loneliness and lonely mood did not differ based on chronic levels of loneliness.

\section{Hypotheses Testing}

Actor-focused hypotheses. A series of one-way ANOVAs were conducted to assess differences among 'lonely' actors $(n=54)$ and their 'connected' counterparts $(n=$ 53), based on the mood induction. Specifically, state loneliness, lonely mood, state selfesteem, perceived event importance, and self-reported levels of perceived responsiveness and the provision of a supportive response during the event-sharing activity, were compared among actors who received the loneliness (vs. social connectedness)

induction. ${ }^{4}$ A summary of hypotheses and results is provided in Table 3.11 and group means are provided in Table 3.1. Analyses assessing differences in the perceived receipt and provision of responsiveness controlled for the extent to which participants knew their lab partner prior to the lab study. ${ }^{5}$

As would be expected given larger group differences $(\mathrm{N}=166)$ and as predicted in Hypothesis 1.0, there were significant group differences in state loneliness $(F(1,105)=$ $6.06, p=.01)$ and marginal group differences in lonely $\operatorname{mood}(F(1,104)=2.76, p=.09)$, such that 'actors' in the loneliness induction condition $(\mathrm{n}=54)$ reported significantly higher levels of state loneliness $(M=8.09, S D=3.02)$ and lonely $\operatorname{mood}(M=2.15, S D=$ 1.22) compared to 'actors' in the connectedness induction condition ( $\mathrm{n}=53$; state loneliness, $M=6.64, S D=3.05$; lonely mood, $M=1.75, S D=1.24)$. Importantly, induction groups did not differ in post-induction negative affect $(F(1.104)=1.99, \mathrm{p}=$ .16). 
Hypothesis 1.1 and 1.2 predicted that participants receiving a loneliness induction would report receiving and providing a less supportive response during the eventdisclosure interaction. There were no group differences in levels of perceived responsiveness $(F(1,105)=2.17, p=.14)$, or the provision of a supportive response $(F(1$, $104)=.03, p=.87)$. Specifically, 'lonely' and 'connected' actors did not differ in the extent to which they reported receiving a supportive response from their lab partner when disclosing a positive event during the event-sharing activity, nor did they differ in the extent to which they reported providing a supportive response to their lab partner's disclosure. Thus, Hypotheses 1.1 and 1.2 were not supported.

Further, Hypothesis 1.3 predicted that participants in the loneliness induction group would report lower state self-esteem and that their shared positive event was less important, compared to individuals in the connectedness induction group. Groups did not differ in their levels of state self-esteem $(F(1,104)=.69, p=.41)$. However, there were marginal group differences in perceptions of event importance $(F(1,104)=3.48, p=$ .07), providing partial support for Hypothesis 1.3. Specifically, 'lonely' actors perceived their positive event as lower in importance $(M=4.08, S D=1.15)$, compared to 'connected' actors $(M=4.44, S D=.71)$.

Actors were also compared on outcomes of the event-sharing activity, specifically levels of positive affect after the interaction, enjoyment of the interaction, and liking towards their lab partner. Hypothesis 1.4 predicted that individuals in the loneliness induction group would report less positive affect after the event-sharing interaction, as well as less enjoyment of the interaction and less liking towards their lab partner. 
Controlling for previous familiarity with lab partner, there were no group differences in post-interaction levels of positive affect $(F(1,104)=.04, p=.85)$, enjoyment of the interaction $(F(1,100)=.80, p=.37)$, or lab partner liking $(F(1,104)=.48, p=.49)$. Thus, Hypothesis 1.4 was not supported.

Despite the absence of group differences in responsiveness (received and provided), post-interaction positive affect, enjoyment of the interaction, and partner liking, there was sufficient justification to test for indirect effects of state loneliness, given significant bivariate associations among state loneliness and key outcomes of the event-sharing interaction (Table 3.0), as well the causal association of the induction condition on state loneliness. Indeed, recent theorists argue that it is possible for an independent variable to affect a dependent variable indirectly, in the absence of a detectable total effect (Hayes, 2009, p. 9). Thus, a series of indirect effects were estimated for state loneliness on associations among actor induction condition (loneliness versus connectedness), and responsiveness (received and provided), positive affect, enjoyment, and lab partner liking (Table 3.2). Indirect effects were estimated via bootstrap-based methods (resample $\mathrm{N}=5,000$ ), and bias-corrected confidence intervals were estimated for point estimates of indirect effects.

It was predicted that the loneliness versus connectedness induction would influence perceived responsiveness (received and provided), post-interaction positive affect, enjoyment of the interaction, and partner liking, via differences in state loneliness (Hypothesis 1.5). Specifically, it was expected that participants receiving the loneliness induction would report greater state loneliness, and thus lower perceived responsiveness 
(received and provided), and lower post-interaction positive affect, enjoyment, and lab partner liking. Actor induction condition significantly affected state loneliness (e.g., $a=$ $1.45, p=.02)$ in all indirect effect models. State loneliness was significantly related to perceived responsiveness $(b=-.07, p=.04)$. However, there was no indirect effect of state loneliness on the actor induction condition-perceived responsiveness association $\left(a^{*} b=.10,95 \%\right.$ BC CI: $\left.[-.01, .32]\right)$. Interestingly, state loneliness was not significantly related to self-reported provision of responsiveness $(b=-.01, p=.39)$. Accordingly, there was no indirect effect of state loneliness on the actor induction condition-provision of responsiveness association $\left(a^{*} b=.02,95 \% \mathrm{BC}\right.$ CI: $\left.[-.04, .12]\right)$.

As predicted in Hypothesis 1.5 and displayed in Table 3.2, there was a significant negative association between state loneliness and post-interaction positive affect $(b=$ $.98, p<.001)$, and a significant indirect effect of state loneliness on the induction condition-positive affect association $\left(a^{*} b=1.41,95 \%\right.$ BC CI: $\left.[.03,3.93]\right)$. Similarly, state loneliness was significantly related to enjoyment of the interaction $(b=-.12, p=$ .01 ), and there was a significant indirect effect of state loneliness on the effect of actor induction condition on enjoyment ( $a^{*} b=.19,95 \% \mathrm{BC} \mathrm{CI}$ : $\left.[.01, .52]\right)$. The association of state loneliness and lab partner liking was marginally significant $(b=-.10, p=.07)$, but there was no indirect effect of state loneliness on actor induction-lab partner liking associations $\left(a^{*} b=.14,95 \% \mathrm{BC} \mathrm{CI}:[-.04, .56]\right)$. Thus, results provided partial support for Hypothesis 1.5, in particular regards to the indirect effects of state loneliness on associations among actor induction condition, and post-interaction positive affect and enjoyment of the event-sharing interaction. 
Hypothesis 1.6 predicted that there would be a significant indirect effect of perceived responsiveness on actor induction condition-event sharing outcome associations (positive affect, enjoyment, partner liking). Similarly, Hypothesis 1.7 predicted that there would be indirect effects of state self-esteem and perceived event importance on actor induction-event sharing outcome associations. However, as displayed in Table 3.2 and demonstrated in tests of Hypothesis 1.1 and 1.2, there were no effects of actor induction condition on perceived responsiveness, the provision of responsiveness, and state self-esteem. Thus, it was not appropriate to test for indirect effects of these variables on the induction condition-event-sharing outcome associations (positive affect, enjoyment, liking). There were marginal effects of induction condition on perceived event importance $(b=.36, p=.06)$. Though perceived event importance did relate to greater positive affect after the event-sharing activity $(b=2.41, .01)$, there were no indirect effects of event importance on the effect of induction condition on postinteraction positive affect $\left(a^{*} b=.97,95 \%\right.$ BC CI: $\left.[-.15,2.99]\right)$. Additionally, perceived event importance did not relate to enjoyment of the interaction $(b=.20, p=.20)$, or partner liking $(b=.21, p=.21)$, and there were no indirect effects of perceived importance on induction-enjoyment $\left(a^{*} b=.07,95 \%\right.$ BC CI: $\left.[-.05, .36]\right)$ and inductionliking associations $\left(a^{*} b=.08,95 \%\right.$ BC CI: $\left.[-.08, .40]\right)$. Thus, Hypotheses 1.6 and 1.7 were not supported.

Lastly, a moderated mediation was conducted to test for conditional indirect effects of state loneliness and perceived responsiveness, based on levels of chronic loneliness (as assessed in the initial online survey). Specifically, the moderating effects of 
chronic loneliness were examined for the indirect effects of state loneliness on induction condition-event-sharing outcome associations (positive affect, enjoyment, lab partner liking). Hypothesis 1.8 predicted that these indirect effects would be stronger for individuals at higher levels of chronic loneliness, for whom state experiences of loneliness may be more salient and retrievable. Bias-corrected confidence intervals were estimated via bootstrapping methods for conditional indirect effects. Chronic loneliness (moderator) was grand-mean centered.

As demonstrated in Table 3.3, the moderating effect of chronic loneliness on paths between induction conduction (independent variable) and state loneliness (mediator; $b=.08, p=.10$ ), and on paths between state loneliness (mediator) and positive affect (outcome; $b=-.03, p=.33$ ), were not significant. However there were significant conditional indirect effects for individuals at mean levels of chronic loneliness $\left(a^{*} b=\right.$ .85 , BC 95\% CI: [.03, 2.47]). Indirect effects were not significant for individuals at below (-1 SD) and above mean levels (+1 SD) of chronic loneliness in this sample.

There was a significant moderating effect of chronic loneliness on the state loneliness-lab partner liking association $(b=.01, p=.02$; Table 3.4). Simple slopes tests (Aiken \& West, 1991) revealed that the association of state loneliness and lab partner liking was significant for participants at mean $(b=-.14, t(106)=-2.15, p=.03)$ and low levels of chronic loneliness $(-1 \mathrm{SD} ; b=-.24, t(106)=-3.84, p<.001)$, though this association was not significant for people at high levels of chronic loneliness (+1 SD; $b=$ $-.04, t(106)=-.59, p=.56)$. As displayed in Figure 4.6, participants at low levels of chronic loneliness reported lower liking towards their lab partner at high levels of state 
loneliness. At low levels of state loneliness, groups did not differ in lab partner liking. There was a significant conditional indirect effect for individuals at below mean levels ($1 \mathrm{SD} ; a^{*} b=.55$, BC $95 \%$ CI: $\left.[.14,1.38]\right)$ and mean levels of chronic loneliness $\left(a^{*} b=\right.$ .17$, BC 95\% CI: $[.01, .36])$. The indirect effects of state loneliness on the induction condition-liking association were not significant for participants at high levels of chronic loneliness.

There was also a significant moderating effect of chronic loneliness on the association between state loneliness and enjoyment of the interaction $(b=-.05, p=.01$; Table 3.5). At low levels of state loneliness, participants low in chronic loneliness enjoyed the interaction more relative to participants at high levels of chronic loneliness. At high levels of state loneliness, individuals low in state loneliness enjoyed interaction less than their more chronically lonely counterparts (Figure 4.7). Additionally, there were significant conditional indirect effects for individuals at below mean $\left(a^{*} b=.65, \mathrm{BC} 95 \%\right.$ CI: $[.18,1.53])$ and mean levels of chronic loneliness $(a * b=.22$, BC 95\% CI: $[.02, .63])$, compared to individuals at higher levels of chronic loneliness, for whom the indirect effect was not significant. Otherwise stated, the indirect effect of state loneliness on the induction condition-enjoyment association, were only significant for individuals who typically experienced low levels to mean levels of chronic loneliness. That is, among individuals at lower levels of chronic loneliness, participants in the loneliness versus connectedness induction enjoyed the event-sharing interaction less, due to higher levels of state loneliness. Simple slopes tests revealed that the association among state loneliness and enjoyment of the interaction was significant for individuals at mean $(b=$ - 
$.18, t(106)=-2.81, p=.01)$ and below mean levels of chronic loneliness (-1 SD; $b=.-$ $.28, t(106)=-3.63, p<.001)$, but not for individuals at high levels of chronic loneliness $(+1 \mathrm{SD} ; b=-.08, t(106)=-1.75, p=.08)$. Though these results provide evidence of moderated mediation, as predicted in Hypothesis 1.8, the direction of the relationships observed were opposite of original hypotheses.

Moderated mediation was also tested for chronic loneliness on the indirect effects of perceived responsiveness. As described in Hypothesis 1.9, it was anticipated that the indirect effects of perceived responsiveness on actor induction condition-event-sharing outcome associations (positive affect, enjoyment, liking), would be stronger for individuals at higher levels of chronic loneliness, who may be more prone to negative patterns of social cognition. As displayed in Tables 3.6-3.8, there were no conditional indirect effects for chronic loneliness on the indirect effects of perceived responsiveness, thus Hypothesis 1.9 was not supported. However, there was a significant moderating effect of chronic loneliness on the association between perceived responsiveness and liking towards lab partner $(b=-.03, p=.04$; Table 3.7). Specifically, when participants perceived their lab partner's response as low in responsiveness, participants at low levels of chronic loneliness liked their partner less, compared to participants at high levels of chronic loneliness. There was no difference in lab partner liking when perceived responsiveness was high (Figure 4.8). Simple slope tests revealed that the association among perceived responsiveness and liking towards lab partner was significant for individuals at mean $(b=.47, t(106)=3.13, p<.001)$ and low levels of chronic loneliness 
$(-1 \mathrm{SD} ; b=.79, t(106)=3.27, p<.001)$, but not for individuals at high levels of chronic loneliness $(+1 \mathrm{SD} ; b=.17, t(106)=.77, p=.44)$

Partner-focused hypotheses. A series of analysis of covariance were conducted to compare perceptions and self-reported behavior among participants interacting with a 'lonely' lab partner (partners in LONELY dyads; $n=57$ ) versus participants interacting with a 'connected' lab partner (partners in CONNECTED dyads; $n=53$ ). As with group comparisons for 'lonely' versus 'connected' actors, previous familiarity with lab partner was included as a covariate. A summary of hypotheses and results is provided in Table 3.11 and group means are provided in Table 3.9. Groups were compared on perceived responsiveness during the event-sharing activity (received and provided), as well as postinteraction levels of positive affect, enjoyment of the interaction, and liking towards lab partner. It was predicted that interaction partners in LONELY versus CONNECTED dyad conditions would report both receiving (Hypothesis 2.1) and providing (Hypothesis 2.2) a less supportive response during the event-sharing interaction. Interaction partners in LONELY versus CONNECTED dyads did not differ in received responsiveness $(F(1$, $107)=2.42, p=.12)$, or self-reported provision of a supportive response $(F(1,107)=$ 2.26, $p=.14)$. Thus, Hypotheses 2.1 and 2.2 were not supported.

It was also predicted that interaction partners in LONELY versus CONNECTED dyads would differ in levels of positive affect, enjoyment, and lab partner liking after the interaction (Hypothesis 2.3). Specifically, it was expected that interaction partners in LONELY dyads would report less positive affect, less enjoyment of the interaction, and like their lab partner less, compared to interaction partners in CONNECTED dyads. 
Results revealed that groups did not differ in post-interaction positive affect $(F(1,107)=$ $.36, p=.55)$, or lab partner liking $(F(1,108)=1.69, p=.20)$. However, there were significant group differences in enjoyment of the interaction $\left(F(1,107)=6.12, p=.02, \eta^{2}\right.$ $=.05)$. Specifically, participants in LONELY dyads enjoyed the interaction much less $(M$ $=8.02, S D=2.08)$, relative to participants in CONNECTED dyads $(M=8.87, S D=$ 1.33). Thus partial support was provided for Hypothesis 2.3

Finally, Hypothesis 2.4 predicted that there would be significant indirect effects of perceive responsiveness on dyad condition (LONELY versus CONNECTED)- eventsharing outcome associations (positive affect, enjoyment, and lab partner liking), among interaction partners. As displayed in Table 3.10, and demonstrated in tests of Hypothesis 2.1, there were no effects of dyad condition on perceived responsiveness. As such, there were no indirect effects of perceived responsiveness on associations among dyad condition and event-disclosure outcomes (positive affect, enjoyment, lab partner liking). Thus, Hypothesis 2.4 was not supported.

\section{Discussion}

The current study drew from multidimensional and transactional models of loneliness to examine consequences of transient loneliness which may interfere with the development and maintenance of close relationships. Specifically, the study examined the extent to which transient, or state experiences of loneliness are consequential to perceptions and behaviors within a lab-based, positive social interaction. Further, the current study investigated the extent to which transient loneliness inhibits individuals from reaping the benefits of important relationship development and maintenance 
processes, as described by a Kashdan et al.'s (2013) positivity-deficit perspective. In taking a positivity-deficit perspective and by focusing on transient loneliness, the study expanded the current empirical study of loneliness, which has primarily focused on negative behavioral processes associated with chronic loneliness. In addition, this study used a novel and ecologically-valid approach by examining the experiences of both 'lonely' individuals and their 'nonlonely' (socially-connected) interaction partners, providing insight into processes influencing others' willingness to interact with lonely people. To our knowledge, this was the first study to date to examine the effects of state loneliness on perceptions and behaviors within the context of a capitalization interaction, thus making a substantive contribution to the existing literature.

Results revealed that participants receiving the loneliness (vs. social connectedness) induction reported higher levels of state loneliness, and thus less positive affect following a positive event-disclosure interaction and less enjoyment of the interaction. Additionally, 'lonely' participants perceived that the positive event they shared with their lab partner was lower in importance, compared to participants who received a social connectedness induction. These results support previously established multidimensional models of loneliness. Though the multidimensional model has been previously used to describe affective, behavioral, and cognitive consequences of chronic loneliness, the current study provided evidence of its application to more fleeting or transient experiences of loneliness. That is, existing correlational research has shown that loneliness is related to less positive affect, more negative perceptions of social interactions, as well as poorer quality social interactions (Hawkley, Burleson, Bernston, 
\& Cacioppo, 2003; Peplau \& Perlman, 1982). In the current study, state loneliness was related to less positive affect and less enjoyment following a lab-based social. This was the first study to date to examine the multidimensional model of loneliness using experimental mood induction methods, thus making a substantive contribution to the existing literature.

Importantly, the affective and perceptual consequences of transient loneliness in the current study were observed within the context of a positive-event disclosure (i.e., capitalization). Previous research has described positive event-disclosure, or capitalization, as a vital relationship development and maintenance process with various intrapersonal and interpersonal outcomes, including positive affect and relationship satisfaction (e.g., Gable et al., 2004). Additionally, research has shown that capitalization is related to less loneliness (Gable \& Reis, 2010). Yet, previous research examining the association between capitalization and loneliness has relied on correlational methods. In utilizing experimental randomization and mood induction, our results provide insight into the causal direction of this relationship. Specifically, results indicate that levels of state loneliness prior to the positive event-disclosure interaction may inhibit individuals from experiencing the benefits of this relationship-building process. As such, results of this study provide support for a positivity-deficit perspective of loneliness, which proposes that loneliness interferes with the ability engage in and benefit from positive social interactions.

Results also support previous research revealing loneliness-related deficits in the perceived importance of shared positive events (Arpin \& Mohr, in preparation). In the 
current study, participants in the loneliness induction group perceived the positive event they shared with their lab partner as less important, compared to participants who received a social connectedness induction. Previous research has provided evidence that lonely persons tend to make more negative attributions for successes and positive events, an attributional-style which may thwart lonely persons' ability to reap the benefits of positive experiences and successes (Anderson, Horowitz, \& French, 1983). Whereas previous work has demonstrate an association between chronic loneliness and the perceived importance of positive events (Arpin \& Mohr, in preparation), findings in the current study show that participants randomly induced to feel lonely versus socially connected experienced similar deficits in perceived event importance.

These findings support the argument that it is the experience of loneliness which contributes to perceptions of event importance, rather than pre-existing differences in attributional-style or related individual difference variables (e.g., depression, pessimism; Sweeney, Anderson, \& Bailey, 1986). Further, that the association among loneliness and perceived event importance has now been observed for chronic and state levels of loneliness provided additional evidence for the positivity dampening effects of this negative-affective experience. As has been previously observed with self-esteem, loneliness may dampen affective reactions to positive events and experiences, thus influencing the extent to which lonely persons can successfully celebrate these events with others (Wood, Heimpel, \& Michela, 2003).

These results also support previous research demonstrating unique patterns of self-disclosure related to loneliness. In particular, work by Solano et al. (1982) provided 
evidence that lonely persons are less likely to disclose intimate information when interacting with a new acquaintance. Though the current study did not assess the perceived level of intimacy of disclosure topics, it is likely that the topics which 'lonely' participants selected to share with their lab partner, differed compared to those selected by their 'socially connected' counterparts (less important among 'lonely' participants vs. important among 'connected' participants). Future work should consider the content of positive-event disclosures among lonely versus nonlonely individuals, which may influence the extent to which loneliness interferes with the intimacy-building properties of capitalization.

Though the results of this study provide support for multidimensional and positivity-deficit models, there was less support for the transactional model of loneliness. Transactional models of loneliness posit that the various symptoms or consequences of loneliness operate on the environment to exacerbate and maintain this negative-affective experience (Ernst \& Cacioppo, 1998). More specifically, this model describes how the perception that one is isolated (i.e., loneliness) increases motivations for selfpreservation, facilitating hyper-vigilance to social threat and negative expectations of social interactions. These expectations then reinforce social withdrawal and further loneliness via behavioral confirmation processes (Chapter I; Cacioppo, Grippo, London, Goosens, \& Cacioppo, 2015). In the context of the current study, the transactional model would be supported had there been significant indirect effects of state self-esteem, perceived event importance, perceived responsiveness, and the provision of responsiveness (all theorized consequences of loneliness) on outcomes of the event- 
disclosure interaction (i.e., positive affect, enjoyment of the interaction, amusement). However, in the current sample, there were no direct effects of induction condition on state self-esteem or responsiveness (perceived and provided), nor were there indirect effects of self-esteem, perceived responsiveness, or perceived event importance on induction-outcome associations, as would be expected in the transactional model.

Whereas previous research has shown a strong correlation among loneliness and self-esteem ( $r=-.72$; Oullet \& Joshi, 1986), it is possible that momentary increases in loneliness are simply not powerful enough to influence levels of self-esteem, which may be more stable. Additionally, though previous research has emphasized the importance of responsiveness in predicting beneficial outcomes of capitalization (e.g., Gable et al., 2004), it is likely that this construct is not as relevant to interactions among strangers. ${ }^{6}$ Early relationship theorists describe responsiveness as based on past experiences of care, validation, and concern within a given relationship, and is thus interpreted within the historical context of the intimate relationship (Reis \& Shaver, 1988). As interaction dyads in the current study were randomly paired strangers, expectations for responsiveness may have been low, compared to interactions among romantic couples or within a previously established relationship. Yet, previous research has utilized stranger dyads in the context of a lab-based capitalization interaction (Reis et al., 2010). However, a key difference between the current study and existing research, however, is that previous studies assessing perceptions of responsiveness within stranger dyads have typically incorporated a confederate, and included a manipulation of different types of response behaviors (e.g., enthusiastic vs. passive). Less is known about the relevance of perceived responsiveness 
within actual, initial social interactions. Future work should consider other mechanisms and consequences of state loneliness, which may vary at the state level and which may be more relevant within initial social interactions. Specifically, variables such as general perceived interpersonal warmth may be more relevant within stranger dyads, and which would likely impact individual and interaction partners' experiences within eventdisclosure interactions among strangers.

It is also possible that participant concerns with self-presentation influenced participants' response to their lab partner's disclosure, as well as self-reports of the provision of responsiveness, thus limiting the variability in participant responses on these variables. Future research should explore the actual response behaviors exhibited by lonely persons in the context of a capitalization interaction. In particular, objective assessments of nonverbal behaviors exhibited by 'lonely' persons during initial social interactions may reveal important differences not captured by self-report assessments.

Additionally, it is possible that the transactional model would be more appropriately tested via longitudinal methods. Indeed, the transactional model proposes that the affective, cognitive, and behavioral consequences of loneliness, and in particular social-behavioral deficits, interact with the environment to exacerbate and maintain this negative-affective experience over time (Ernst \& Cacioppo, 1998). In relation to transient loneliness, this transactional process likely unfolds over multiple social interactions, which would limit the extent to which it is observable in the context of a single, lab-based interaction. Future research should apply longitudinal or experience-sampling methods to 
assess how the social-perceptual and behavioral consequences of transient loneliness relate to later levels of loneliness, and increases in loneliness over time.

Results also revealed interesting patterns of individual differences in the indirect effects of state loneliness on positive affect, enjoyment, and liking outcomes, based on chronic experiences of loneliness. Specifically, the indirect effects of state loneliness on induction-outcome associations were only significant for individuals at mean and below mean levels of chronic loneliness, as assessed in the initial online survey. Further, the effects of state loneliness (mediator) on enjoyment of the interaction and positive affect were moderated by chronic loneliness. As state loneliness increased, individuals at lower levels of chronic loneliness enjoyed the interaction less and reported less liking towards their lab partner, relative to individuals at higher levels of chronic loneliness. There was a similar interaction effect of chronic loneliness on the perceived responsiveness-partner liking association (within the induction condition-perceived responsiveness-liking indirect effect model), such that as perceived responsiveness increased, individuals low in chronic loneliness reported liking their lab partner more relative to individuals at low levels of chronic loneliness. These results suggest that state loneliness, within a lab-based setting, may be more powerful and salient for individuals at low levels of chronic loneliness.

Interestingly, these patterns of individual differences are opposite to the original hypotheses, which proposed that the indirect effects of state loneliness and perceived responsiveness would be stronger for individuals at higher levels of chronic loneliness. However, results are actually consistent with previous findings regarding individual 
differences in daily loneliness-solitary alcohol use associations, based on levels of perceived social support. Specifically, study one in this dissertation (Arpin et al., 2015) showed that daily associations among state loneliness and solitary alcohol use were stronger for individuals at higher levels of social support, counter to traditional stressbuffering models. In line with Arpin et al. (2015), the current study revealed that it was individuals at lower levels of chronic loneliness whose experience within the eventdisclosure interaction was more negatively impacted by the loneliness induction and subsequent differences in state loneliness.

Further, though in contrast to our original hypotheses, these results are consistent with traditional cognitive-discrepancy models of loneliness. The cognitive-discrepancy model describes loneliness as occurring upon a perceived discrepancy of what is desired or expected in relationships, and what is received or felt in the moment (Paloutzien \& Janigian, 1987; Russell et al., 1980). As individuals at low levels of chronic loneliness typically feel socially connected, activating memories of loneliness via the induction activity may have been more salient and consequential, given the contrast of state loneliness to average feelings of connectedness. For these individuals, the consequences of loneliness, such as increased vigilance to social threat, may be more powerful in the moment in order to motivate social reconnection, as proposed by the evolutionary model of loneliness (Qualtner et al., 2015).

However, as previously articulated, it is also likely that rather than motivating reaffiliation, the consequences of transient loneliness (e.g., enjoyment of positive social interactions) influence the extent to which persons low in chronic loneliness seek out and 
benefit from similar social interactions in the future. Indeed, past research and theory has described how experiences with past interaction partners can influence experiences within current social interactions, by shaping the filter through which current and future social interactions are interpreted (e.g., Clark \& Lemay, 2010; Reis \& Shaver, 1988). Individuals who typically experience low levels of loneliness may have higher expectations for social interactions, particularly in the context of self-disclosure. Thus, for these individuals, negative social experiences may have a more lasting impact, compared to individuals who have lower expectations when interacting with others.

Future work should consider the extent to which individuals low versus high in chronic loneliness exhibit increased motivation for social reaffiliation (as proposed by the evolutionary model of transient loneliness; Qualtner et al., 2015). Researchers should also investigate specific social events and social contexts which relate to increased transient loneliness in vivo, and which may indirectly influence experiences within social interactions (via increases in transient loneliness). Such work would inform the development of intervention and prevention strategies which could be used to combat feelings of loneliness in the moment (e.g., reducing negative self-talk, changing maladaptive social cognitions; Masi, Chen, Hawkley, \& Cacioppo, 2013) for individuals at high and low levels of chronic loneliness.

Results also motivate future work to continue exploring affective, behavioral, and cognitive consequences of transient loneliness more generally, with particular focus on the consequences of transient loneliness among individuals who typically experience high levels of support. Although transient loneliness is a commonly reported experience, the 
majority of research to date has focused on prolonged experiences of loneliness which are related to severe psychological and physiological health consequences. A greater focus on behavioral processes related to transient loneliness would provide insight into the developmental process through which transient loneliness may develop into more chronic levels over time, as proposed by the transactional model.

Results of the current study also provide insight into the social consequences of loneliness among those encountering lonely interaction partners. Specifically, 'connected' lab partners, interacting with another 'connected' participant reported greater enjoyment of the lab interaction, compared to those interacting with a 'lonely' individual. These results are in line with previous work showing that nonlonely individuals often rate 'lonely' persons as less enjoyable interaction partners, and as less desirable future friends (Bell, 1985; Jones et al., 1982), an effect which some researchers describe as the social stigmatization of loneliness (Lau \& Gruen, 1992). Yet, previous work examining nonlonely individuals' perceptions of lonely others have utilized vignettes, wherein participants read about a explicitly lonely versus nonlonely participant, and rate this individual on outcomes such as interpersonal attractiveness and desirability as a future friend (Rotenberg \& Kmill, 1992). In the current study, interaction partners were not aware that their lab partner was 'lonely' versus 'socially connected'. Rather, ratings of enjoyment were derived from actual interactions with another participant, thus providing for a more ecologically-valid assessment of partner perceptions of 'lonely' versus 'nonlonely' others. 
Further, previous research exploring the stigmatization of loneliness has focused on nonlonely others' perceptions of more chronically lonely individuals. That interaction partners of 'lonely' persons reported enjoying the event-sharing interaction less, compared to interaction partners of 'socially-connected' individuals, extends existing research on the social stigma of loneliness to include fleeting, transient forms of this negative-affective experience. In doing so, findings provide insight into one potential process through which transient loneliness may develop into more chronic levels over time. That is, individuals may be less likely to seek out future social interactions with even transiently lonely persons, thus preventing both individuals from benefitting from future engagement in this vital relationship development and maintenance process.

Interestingly, there were no differences in lab partner liking or post-interaction positive affect among interaction partners in LONELY versus CONNECTED dyads. In addition, as will be described below, lab partners did not differ in the extent to which they perceived 'lonely' versus 'connected' participants as responsive during the eventdisclosure interaction. Yet, previous work has provided evidence of the beneficial effects of mutual self-disclosure on liking towards others (Sprecher, Treger, \& Wondra, 2012). Thus, it is possible that levels of positive affect and liking among interaction partners in this sample were positively impacted by the mutual nature of the disclosure interaction. Indeed, ratings of lab partner liking among interaction partners were positively biased in this sample, with the majority of participants (65\%) providing above mean ratings of partner liking. More research is needed to explore mechanisms through which interaction partners are negatively impacted by social interactions with lonely versus nonlonely 
others. Future work should also consider the extent to which interacting with a lonely person influences levels of loneliness among interaction partners, as proposed by the induction hypothesis, in the unique context of disclosure interactions (Cacioppo et al., 2009).

\section{Limitations}

Though the current study makes a substantive contribution to the existing loneliness and close relationships literature, there are several methodological limitations which should be noted. The first limitation relates to the use stranger-participant dyads. As described in Reis et al. (2010), in real life it is likely that capitalization interactions and experiences occur among individuals in close relationships (i.e., intimate relationships or friendship). Thus, responses to capitalization attempts would occur and be interpreted in the historical context of the relationship (Reis \& Shaver, 1988), which would function as an "interpretative filter" for perceptions and experiences during the disclosure interaction (p. 378). Though the current study incorporated a 'getting-to-knowyou' activity at the beginning of the lab session to facilitate familiarity among participants, it is likely that the level of intimacy of positive-event disclosures, and thus perceptions of responsiveness within the interaction, would have been greater had the interaction occurred among friends or intimate partners. Future work should examine role of transient loneliness within positive event-disclosure interactions within previously established relationships, which may reveal unique effects of state loneliness on perceptions of responsiveness in the moment, relative to preexisting expectations of responsiveness in the relationship. 
In light of the limitation described above, it should be noted that a key contribution of the current study was the potential to establish evidence of a causal link among loneliness and positivity deficits within disclosure interactions. As this was the first study to utilize a loneliness induction paradigm in the context of a lab-based capitalization interaction, the decision to use stranger dyads was based on a methodological concern that a pre-established relationship among participants might buffer the effects of the loneliness mood induction. Additionally, a central focus of this study was to examine whether loneliness and related social and perpetual deficits interfere with relationship development strategies. That capitalization and self-disclosure more generally are key processes in the development of close relationships and intimacy made examining the effects of loneliness on initial disclosure interactions an important first step. Results of the current study revealed interesting group differences and indirect effects of state loneliness in relation to key outcomes of the capitalization interaction (positive affect, enjoyment of the interaction, liking towards lab partner). Yet, proposed effects of loneliness on perceived responsiveness (received and provided) were not observed, thus leaving much room for future work to explore these associations within different social contexts (e.g., intimate relationships, friendships, etc.), within which expectations for responsiveness within a self-disclosure interaction may be higher.

A second limitation, which may influence the generalizability of our results, is the use of an undergraduate student sample and the focus on female interaction. It should be noted that, unlike typical college student samples used in social psychological lab research, the current study comprised participants from a largely non-traditional 
undergraduate-student sample. Indeed, sample characteristics differed from those in the typical college-student sample, particularly in terms of average age $(M=25.09, S D=$ 8.62 , range $=18-64)$, and percent employed $(59 \%)$. Thus, in terms of age and diverse life experiences, the sample used in the current study was slightly more diverse than typical undergraduate student populations, and thus more generalizable to the general population.

Further, as this was the first study to investigate the effects of state loneliness within positive-event disclosure interactions, it was important to use same-gender dyads to control for potential confounds (e.g., gender differences in self-disclosure behavior, interpersonal attraction, communication-style, and social support provision; e.g., Dindia \& Allen, 1992), as well as previously established gender differences in self-labeling measures of loneliness and lonely mood (Borys \& Perlman, 1985). Though there were no gender differences in the induction effects, as demonstrated within exploratory analyses in the pilot study (Appendix A), it is possible that the processes assessed in the current study differ for men and women. Indeed, existing studies examining the effects of chronic loneliness on self-disclosure behavior has compared same and opposite gender interactions, revealing interesting differences in the extent to which lonely persons exhibit differences in intimate disclosure behavior when sharing with a same versus opposite gender partner (e.g., Solano et al., 1982). Thus, future work should investigate the role of state loneliness in capitalization interactions among male dyads, as well as among interaction partners of the opposite gender.

An additional limitation to generalizability relates to the eligibility criteria for the lab study. Due to ethical concerns for the content of the loneliness induction (i.e., "Think 
back on a time when you felt like you didn't belong... when you had no one to relate to"), individuals at risk of depression were screened out of the lab study. As chronic loneliness and depression are highly correlated, screening procedures may have limited our sample to individuals at less extreme levels of chronic loneliness. Indeed, mean levels of chronic loneliness in this sample were low $(M=33.33, S D=9.92)$ in comparison to previous research with college student samples ( $M=40, S D=9.50$; Russell, 1996). However, these lower levels of loneliness may be accounted for by the diverse age range of this sample (25-64). Interestingly, research has shown that the relationship of age and loneliness is curvilinear, with young adults and the elderly reporting high levels of loneliness, and those in mid-life reporting lower levels of loneliness (Perlman, 1991). Future work should continue to explore the intrapersonal and interpersonal consequences of transient loneliness among individuals at varying levels of chronic loneliness through the use of daily-process and experience sampling research. Such methods may address some of the ethical concerns of lab-based mood induction, particularly for individuals at high levels of chronic loneliness and for those at risk of depression.

Another limitation relates to sample size and statistical power. Though data did not exhibit non-independence and analyses were at the individual- rather than dyadiclevel of analyses, the final sample size $(\mathrm{N}=166)$ may have limited statistical power for tests of indirect effects and moderated mediation analyses. Concerns with statistical power and resulting robustness of analyses were addressed via the utilization of bootstrapping and estimation of bias-corrected confidence intervals for indirect effects and conditional indirect effects. Recent work has demonstrated that bootstrapping offers 
various advantages to the estimation of indirect effects over the traditional approaches, including the absence of strict distributional assumptions of normality, particularly with smaller sample sizes (Preacher \& Hayes, 2008). Further, Fritz and MacKinnon (2007) describe samples of $\mathrm{N}=100-200$ as providing sufficient statistical power when testing indirect effects wherein paths $a$ and $b$ are medium to large effects. According to these conventions, many of the indirect effect models for state loneliness on actor induction condition-capitalization outcome associations were sufficiently powered with the current sample size. However, given the small effect of dyad condition on partner capitalization outcomes, it is possible that partner group comparisons and subsequent indirect effects were underpowered. Future work should seek to replicate the current findings with a larger sample, which may provide greater statistical power to test for group differences among interaction partners.

A final limitation to this study relates to issues of external validity common among experimental-lab research. Though experimental-lab methods have the advantage of strong internal validity and experimental control, the lab space is often criticized for being unrealistic and not representative of the real world. Along with this, the social interactions that take place in the lab are likely less genuine and natural compared to those which occur in everyday life. Yet, as this was the first study to investigate the consequences of transient in the context of a capitalization interaction, it was important to isolate the effects of loneliness in the lab, controlling for pre-existing differences in loneliness and related psychological experiences (e.g., depression) via randomized mood induction. In addition to continuing to explore the effects of transient loneliness in the 
lab, future work should probe the observed patterns of associations in the real world and within established personal relationships, via daily-process and experience-sampling research. Indeed, as described in Cialdini's (1997) full cycle model of research, utilizing lab-based experimental research to inform applied, correlational methods (and vice versa) is important in gaining a full understanding of social psychological phenomena.

\section{Conclusion}

In sum, the current study investigated the effects of transient loneliness on individual and interaction partner perceptions within a positive event-disclosure interaction. Results provide support for multidimensional models of loneliness, and expand the current understanding of loneliness to include deficits within positive relationship processes. By incorporating individual and interaction-partner reports, this study offered valuable insight into the interpersonal consequences of transient loneliness in the early stages of relationship development. Further, individual differences suggest that transient loneliness may be particularly consequential among individuals who typically feel socially-connected. Further, results challenge theoretical assumptions about the role of transient loneliness in motivating social reconnection (Qualter et al., 2015). That is, the current study provided evidence of causal links among transient loneliness and social-perceptual deficits which may negatively impact future event-disclosure interactions. Lastly, results provide a foundation from which future work can continue to explore the consequences of transient loneliness, which may negatively impact social interactions among those who feel lonely in the moment and those with whom they interact. 


\section{References}

Aiken, L. S., \& West, S. G. (1991). Multiple regression: Testing and interpreting interactions. Newbury Park, CA: Sage.

American Psychiatric Association (2000). Diagnostic and statistical manual of mental disorders. (4 ${ }^{\text {th }}$ ed.) Washington, DC: American Psychiatric Association.

Arpin, S. N., \& Mohr, C. D. (in preparation). No one to turn to in times of joy: The inhibiting effects of loneliness on positive-event disclosure.

Arpin, S. N., Mohr, C. D, \& Brannan, D. (2015). Having friends but feeling lonely: A daily-process examination of transient loneliness, drinking behavior, and the influence of interpersonal relationships. Personality and Social Psychology Bulletin, 40, 615-628.

Baldwin, M. W. (1992). Relational schemas and the processing of social information. Psychological Bulletin, 112(3), 461.

Bargh, J. A., \& Chartrand, T. L. (2000). The mind in the middle. Handbook of research methods in social and personality psychology, 253-285.

Baumeister, R. F., \& Leary, M. R. (1995). The need to belong: desire for interpersonal attachments as a fundamental human motivation. Psychological Bulletin, 117(3), 497.

Bekhet, A. K., \& Zauszniewski, J. A. (2012). Mental health of elders in retirement communities: is loneliness a key factor? Archives of Psychiatric Nursing, 26(3), 214-224. 
Bell, R. A. (1985). Conversational involvement and loneliness. Communications Monographs, 52(3), 218-235.

Blouin, A. G., Perez, E. L., \& Blouin, J. H. (1988). Computerized administration of the diagnostic interview schedule. Psychiatry Research, 23(3), 335-344.

Bollen, K., \& Lennox, R. (1991). Conventional wisdom on measurement: A structural equation perspective. Psychological Bulletin, 110(2), 305.

Borys, S., \& Perlman, D. (1985). Gender differences in loneliness. Personality and Social Psychology Bulletin, 11(1), 63-74.

Burisch, M. (1984). Approaches to personality inventory construction: A comparison of merits. American Psychologist, 39, 214-227.

Cacioppo, J. T., Fowler, J. H., \& Christakis, N. A. (2009). Alone in the crowd: the structure and spread of loneliness in a large social network. Journal of Personality and Social Psychology, 97(6), 977.

Cacioppo, J. T., Hawkley, L. C., Ernst, J. M., Burleson, M., Berntson, G. G., Nouriani, B., \& Spiegel, D. (2006). Loneliness with a nomological net: An evolutionary perspective. Journal of Research in Personality, 40, 1054-1085.

Chelune, G. J., Sultan, F. E., \& Williams, C. L. (1980). Loneliness, self-disclosure, and interpersonal effectiveness. Journal of Counseling Psychology, 27(5), 462.

Clark, M.S. \& Lemay, E.P. (2010). Close relationships. Handbook of Social Psychology.

Cohen, J. (1988). Statistical power analysis for the behavioral sciences $\left(2^{\text {nd }}\right.$ ed.) Hillsdale, NJ: Erlbaum. 
Collins, N. L., \& Miller, L. C. (1994). Self-disclosure and liking: a meta-analytic review. Psychological Bulletin, 116(3), 457.

Cozby, P. C. (1973). Self-disclosure: a literature review. Psychological Bulletin, 79(2), 73.

Cronbach, L. J. (1951). Coefficient alpha and the internal structure of tests. Psychometrika, 16(3), 297-334.

Dindia, K., Fitzpatrick, M. A., \& Kenny, D. A. (1997). Self-Disclosure in Spouse and Stranger Interaction A Social Relations Analysis. Human Communication Research, 23(3), 388-412.

Dindia, K., \& Allen, M. (1992). Sex differences in self-disclosure: a metaanalysis. Psychological Bulletin, 112(1), 106.

Fritz, M. S., \& MacKinnon, D. P. (2007). Required sample size to detect the mediated effect. Psychological Science, 18(3), 233-239.

Gable, S. L., \& Reis, H. T. (2010). Good news! Capitalizing on positive events in an interpersonal context. Advances in Experimental Social Psychology, 42, 195-257.

Gable, S. L., Gonzaga, G. C., \& Strachman, A. (2006). Will you be there for me when things go right? Supportive responses to positive event disclosures. Journal of Personality and Social Psychology, 91(5), 904.

Gable, S. L., Reis, H. T., Impett, E. A., \& Asher, E. R. (2004). What do you do when things go right? The intrapersonal and interpersonal benefits of sharing positive events. Journal of Personality and Social Psychology, 87(2), 228. 
Ghiselli, E. E., Campbell, J. P., \& Zedeck, S. (1981). Measurement theory for the behavioral sciences. San Francisco: Freeman.

Glaser, R., Kiecolt-Glaser, J. K., Speicher, C. E., \& Holliday, J. E. (1985). Stress, loneliness, and changes in herpes virus latency. Journal of Behavioral Medicine, 8(3), 249-260.

Hawkley, L. C., Burleson, M. H., Bernston, G. G., \& Cacioppo, J. T. (2003). Loneliness in everyday life: Cardiovascular activity, psychosocial context, and health behavior. Journal of Personality and Social Psychology, 85(1), 105-120.

Hayes, A. F. (2009). Beyond Baron and Kenny: Statistical mediation analysis in the new millennium. Communication Monographs, 76(4), 408-420.

Heatherton, T. F. \& Polivy, J. (1991). Development and validation of a scale for measuring state self-esteem. Journal of Personality and Social Psychology, 60, 895-910.

Heatherton, T. F., Wyland, C. L., \& Lopez, S. J. (2003). Assessing self-esteem. Positive Psychological Assessment: A Handbook of Models and Measures, 219-233.

Heinrich, L. M., \& Gullone, E. (2006). The clinical significance of loneliness: A literature review. Clinical Psychology Review, 26(6), 695-718.

Holmes, E. A., Mathews, A., Dalgleish, T., \& Mackintosh, B. (2006). Positive interpretation training: Effects of mental imagery versus verbal training on positive mood. Behavior Therapy, 37(3), 237-247.

Howell, David C. (2010). Statistical Methods for Psychology (7th ed). Belmont, CA: Duxbury Press. 
EXPERIMENTAL INDUCTION OF LONELINESS

IBM Corp. Released 2013. IBM SPSS Statistics for Windows, Version 22.0. Armonk, NY: IBM Corp.

Ikegami, T. (2002). State self-esteem as a moderator of negative mood effects on person impression. Journal of Experimental Social Psychology, 38(1), 1-13.

Jallais, C., \& Gilet, A. L. (2010). Inducing changes in arousal and valence: Comparison of two mood induction procedures. Behavior Research Methods, 42(1), 318-325

Johnson, J. G., \& Miller, S. M. (1990). Attributional, life-event, and affective predictors of onset of depression, anxiety, and negative attributional style. Cognitive Therapy and Research, 14(4), 417-430.

Jones, W. H., Hobbs, S. A., \& Hockenbury, D. (1982). Loneliness and social skill deficits. Journal of Personality and Social Psychology, 42(4), 682.

Joseph, S., \& Lewis, C. A. (1998). The Depression-Happiness Scale: Reliability and validity of a bipolar self-report scale. Journal of Clinical Psychology, 54(4), 537544.

Joseph, S., Linley, P. A., Harwood, J., Lewis, C. A., \& McCollam, P. (2004). Rapid assessment of well-being: The Short Depression-Happiness Scale (SDHS). Psychology and psychotherapy: Theory, research and practice, 77(4), 463-478.

Jourard, S. M. (1964). The transparent self: Self-disclosure and well-being (No. 17). Princeton, NJ: Van Nostrand.

Kashdan, T. B., Ferssizidis, P., Farmer, A. S., Adams, L. M., \& McKnight, P. E. (2013). Failure to capitalize on sharing good news with romantic partners: Exploring 
EXPERIMENTAL INDUCTION OF LONELINESS

positivity deficits of socially anxious people with self-reports, partner-reports, and behavioral observations. Behaviour Research and Therapy, 51(10), 656-668.

Kashdan, T. B., Week, J. W., \& Savostyanova, A. A. (2011). Whether, how, and when social anxiety shapes positive experiences and events: A self-regulatory framework and treatment implications. Clinical Psychology Review, 31, 786-799.

Kenny, D. A., Kashy, D. A., \& Cook, W. L. (2006). Dyadic Data Analysis. Guilford Press.

Langston, C. A. (1994). Capitalizing on and coping with daily-life events: Expressive responses to positive events. Journal of Personality and Social Psychology, 67(6), 1112

Larsen, R. J., \& Fredrickson, B. L. (1999). Measurement issues in emotion research. In D. Kahneman, E. Diener, \& N. Schwarz (Eds.), Well-being: The foundations of hedonic psychology (pp. 40-60). New York: Russell Sage Foundation.

MacGregor, J. C., Fitzsimons, G. M., \& Holmes. J. G. (2013). Perceiving low self-esteem in close others impedes capitalization and undermines the relationship. Personal Relationships, 20(4), 690-705.

Maisel, N. C., Gable, S. L., \& Strachman, A. (2008). Responsive behaviors in good times and in bad. Personal Relationships, 15(3), 317-338.

Masi, C. M., Chen, H. Y., Hawkley, L. C., \& Cacioppo, J. T. (2011). A meta-analysis of interventions to reduce loneliness. Personality and Social Psychology Review, 15(3). 
EXPERIMENTAL INDUCTION OF LONELINESS

Mattick, R. P., \& Clarke, J. C. (1998). Development and validation of measures of social phobia scrutiny fear and social interaction anxiety. Behaviour Research and Therapy, 36(4).

Mills, C., \& D'Mello, S. (2013). On the validity of the autobiographical emotional memory task for emotion induction. PloS one, 9(4).

Myers, D. G., \& Diener, E. (1995). Who is happy? Psychological Science, 6(1), 10-19.

Ouellet, R., \& Joshi, P. (1986). Loneliness in relation to depression and selfesteem. Psychological Reports, 58(3), 821-822.

Page-Gould, E., Mendoza-Denton, R., \& Tropp, L. R. (2008). With a little help from my cross-group friend: reducing anxiety in intergroup contexts through cross-group friendship. Journal of Personality and Social Psychology, 95(5), 1080.

Peplau, L. A., \& Perlman, D. (1979). Blueprint for a social psychological theory of loneliness. Love and attraction, 101-110.

Perlman, D. (1991). Age differences in loneliness. A meta-analysis. Vancouver, Canada: University of British Columbia. (ERIC Document Reproduction Service No. ED 326767).

Perlman, D., \& Peplau, L. A. (1984). Loneliness research: A survey of empirical findings. In L.A. Peplau \& S. Goldston (Eds.), Preventing harmful consequences of severe and persistent loneliness, (pp. 13-46). U.S. Government Printing Office: DDH Publication No. (ADM) 84-1312. 
Preacher, K. J., \& Hayes, A. F. (2008). Asymptotic and resampling strategies for assessing and comparing indirect effects in multiple mediator models. Behavior Research Methods, 40(3), 879-891.

Pressman, S. D., Cohen, S., Miller, G. E., Barkin, A., Rabin, B. S., \& Treanor, J. J. (2005). Loneliness, social network size, and immune response to influenza vaccination in college freshmen. Health Psychology, 24(3), 297.

Qualter, P., Vanhalst, J., Harris, R., Van Roekel, E., Lodder, G., Bangee, M., Maes, M., \& Verhagen, M. (2015). Loneliness across the life span. Perspectives on Psychological Science, 10(2), 250-264.

Reis H.T. University of Rochester (2003). A self-report measure of perceived partner responsiveness. Unpublished data.

Reis, H. T., Maniaci, M. R., Caprariello, P. A., Eastwick, P. W., \& Finkel, E. J. (2011a). Familiarity does indeed promote attraction in live interaction. Journal of Personality and Social Psychology, 101, 557-570.

Reis, H. T., \& Shaver, P. (1988). Intimacy as an interpersonal process. In S. Duck, E. F.Hay, D.F., S. E. Hobfoll, W. Ickes, B. M. Montgomery (Eds), Handbook of personal relationships: Theory, research and interventions, (pp. 367-389). Oxford, England: John Wiley \& Sons.

Reis, H. T., Clark, M. S., \& Holmes, J. G. (2004). Perceived partner responsiveness as an organizing construct in the study of intimacy and closeness. Handbook of closeness and intimacy, 201-225 
Reis, H. T., Smith, S. M., Carmichael, C. L., Caprariello, P. A., Tsai, F. F., Rodrigues, A., \& Maniaci, M. R. (2010). Are you happy for me? How sharing positive events with others provides personal and interpersonal benefits. Journal of Personality and Social Psychology, 99(2), 311.

Rosenberg, M. (1965). The measurement of self-esteem. Society and the Adolescent SelfImage, 297, 307.

Rotenberg, K. J. (1997). Loneliness and the perception of the exchange of disclosures. Journal of social and clinical psychology, 16(3), 259-276.

Rotenberg, K. J., \& Flood, D. (1999). Loneliness, dysphoria, dietary restraint, and eating behavior. International Journal of Eating Disorders, 25(1), 55-64.

Rotenberg, K. J., Gruman, J. A., \& Ariganello, M. (2002). Behavioral confirmation of the loneliness stereotype. Basic and Applied Social Psychology, 24(2), 81-89.

Russell, D. W. (1996). UCLA Loneliness Scale (Version 3): Reliability, validity, and factor structure. Journal of Personality Assessment, 66(1), 20-40.

Sarason, B. R., Sarason, I. G., \& Gurung, R. A. R. (2001). Close personal relationships and health outcomes: A key to the role of social support. In B. R. Sarason \& S. Duck (Eds.), Personal relationships: Implications for clinical and community psychology (pp. 15-41). West Sussex, England: Wiley \& Sons, LTD.

Shankur, A., McMunn, A., Banks, J., \& Steptoe, A. (2011). Loneliness, social isolation, and behavioral and biological health indicators in older adults. Health Psychology, 30(4), 377-385. 
Sheldon, K. M., \& Gunz, A. (2009). Psychological needs as basic motives, not just experiential requirements. Journal of Personality and Social Psychology, 77(5), $1467-1492$.

Shrout, P. E., \& Bolger, N. (2002). Mediation in experimental and non-experimental studies: new procedures and recommendations. Psychological Methods, 7(4), 422.

Smith, S. M., \& Reis, H. T. (2012). Perceived responses to capitalization attempts are influenced by self-esteem and relationship threat. Personal Relationships, 19(2), 367-385.

Sprecher, S., Treger, S., \& Wondra, J. D. (2013). Effects of self-disclosure role on liking, closeness, and other impressions in get-acquainted interactions. Journal of Social and Personal Relationships, 30(4), 497-514.

Solano, C. H., Batten, P. G., \& Parish, E. A. (1982). Loneliness and patterns of selfdisclosure. Journal of Personality and Social Psychology, 43(3), 524.

Strube, M. J. (1991). Small sample failure of random assignment: A further examination. Journal of consulting and clinical psychology, 59(2), 346.

Watson, D., Clark, L. A., \& Tellegen, A. (1988). Development and validation of brief measures of positive and negative affect: The PANAS scales. Journal of Personality and Social Psychology, 54(6), 1063-1070.

Westermann, R., Spies, K., Stahl, G., \& Hesse, F. W. (1996). Relative effectiveness and validity of mood induction procedures: A meta-analysis. European Journal of Social Psychology, 26(4), 557-580. 
Wheeless, L. R., \& Grotz, J. (1977). The measurement of trust and its relationship to selfdisclosure. Human Communication Research, 3(3), 250-257.

Wildschut, T., Sedikides, C., Routledge, C., Arndt, J., \& Cordaro, F. (2010). Nostalgia as a repository of social connectedness: the role of attachment-related avoidance. Journal of Personality and Social Psychology, 98(4), 573.

Wilson, C., \& Moulton, B. (2010). Loneliness among older adults: A national survey of adults 45+. Prepared by Knowledge Networks and Insight Policy Research. Washington, DC: AARP. Nonartists, (45).

Wilson, T. D., Aronson, E., \& Carlsmith, K. (2010). The art of laboratory experimentation. Handbook of Social Psychology.

Wittenberg, M. T., \& Reis, H. T. (1986). Loneliness, social skills, and social perception. Personality and Social Psychology Bulletin, 12(1), 121-130.

Wood, J. V., Heimpel, S. A., \& Michela, J. L. (2003). Savoring versus dampening: Selfesteem differences in regulating positive affect. Journal of Personality and Social Psychology, 85(3), 566.

Zhou, X., Sedikides, C., Wildschut, T., \& Gao, D. G. (2008). Counteracting Loneliness. On the Restorative Function of Nostalgia. Psychological Science, 19(10), 10231029. 


\section{Footnotes}

${ }^{1}$ Results of the pilot revealed that the effects of the loneliness induction were reduced to marginal significance when controlling for general negative affect. However this attenuation would be expected given the strong correlation between lonely mood and negative affect $(r=.39, p=.002)$. Loneliness itself is a negative emotional experience, associated with increases in other forms of negative affect (e.g., sadness, boredom, anxiety; Perlman \& Peplau, 1984). Further, that the effects of the induction on lonely $\operatorname{mood}\left(\eta^{2}=.08\right)$ were larger than those observed for general negative affect $\left(\eta^{2}=.06\right)$ supports the use of the induction procedure as an effective method for inducing feelings of loneliness, beyond general levels of negative affect. However, given these results, it was determined necessary to control for negative affect when testing the effects of the induction procedure in the primary study.

${ }^{2}$ For lab sessions where only one of the two participants showed up, the present participant was led through the pilot study procedure so that they could participate in the study and receive their extra credit without penalty.

${ }^{3}$ Bollen and Lennox (1991) differentiate among multiple indicators as effects of a latent construct, and multiple indicators as causes of a latent construct. In the former case (indicators as effects of a construct), testing for internal consistency among items would be important. In the latter case (multiple indicators as causes of a construct), however, the indicators define the latent construct, and thus would not necessarily be expected to correlate. Each item in the 4-item version of the Perceived Responses to Capitalization Attempts Scale (Gable et al., 2006) represents a distinct category of responsiveness (i.e., active-constructive, passive-constructive, active-destructive, and passive-destructive). Further, the 'responsiveness' composite derived from these items was more so a ratio of positive to negative responses, rather than an aggregation of items. Further, only two of items in the 4-item PRCA were correlated in this sample ("Reacted enthusiastically to this event" and "Seemed disinterested"; $r=-.46, p<.001)$. Thus, it was not appropriate to test for internal consistency among these items.

${ }^{4}$ Given the strong effect of the induction on state loneliness $\left(\eta^{2}=.05\right)$ versus lonely mood $\left(\eta^{2}=.04\right)$ and the independent effects of the induction on state loneliness above and beyond levels of negative affect, state loneliness was used in subsequent analyses beyond the manipulation check to increase statistical power in actor group comparisons (state loneliness and lonely mood were strongly correlated, $r=.70, p<.01)$. Additionally, as lonely mood was an item on the PANAS, from which negative affect and positive affect composites were derived, utilization of the state loneliness measure as a mediator in indirect effect and moderated mediation models (particularly in models with positive affect outcomes) addressed some concern with common-method bias, as well as a singleitem assessment of loneliness (Ghiselli, Campbell, \& Zedeck, 1981). 
${ }^{5}$ Results were the same when the 'previous familiarity' control was excluded from each model. After the exclusion of participants who knew one another very well (as described on p. 117), $4 \%$ of participants indicated that they had interacted with their lab partner once or twice in class, but did not talk on a regular basis; $93 \%$ indicated that they had never interacted with their lab partner before.

${ }^{6}$ It should be noted that exploratory analyses revealed marginal group differences among actors $(F(1,105)=3.15, p=.08)$ in one facet of responsiveness (i.e., "Reacted enthusiastically to this event"), with 'lonely' actors reporting lower levels of an enthusiastic response $(M=4.00, S D=.93)$, relative to 'connected' actors $(M=4.30, S D=$ .82). Similarly, there were marginal group differences among interaction partners in a single-item facet of responsiveness $(F(1,108)=2.85, p=.09)$, with interaction partners in LONELY dyads reporting lower levels of an enthusiastic response $(M=4.02, S D=$ $.94)$, relative to interaction partners in CONNECTED dyads $(M=4.30, S D=.82)$.

However, concerns with poor reliability of single-item measures did not warrant the use of this item as an assessment of responsiveness in tests of group differences (H1.1, H2.1), indirect effects (H1.5, H1.6, H2.4), and moderated mediation (H1.9). Additionally, previous use of the 4-item version of the Perceived Responsiveness to Capitalization Attempts measure (Gable et al., 2006) has assessed responsiveness via a single composite responsiveness, as computed in this study. 


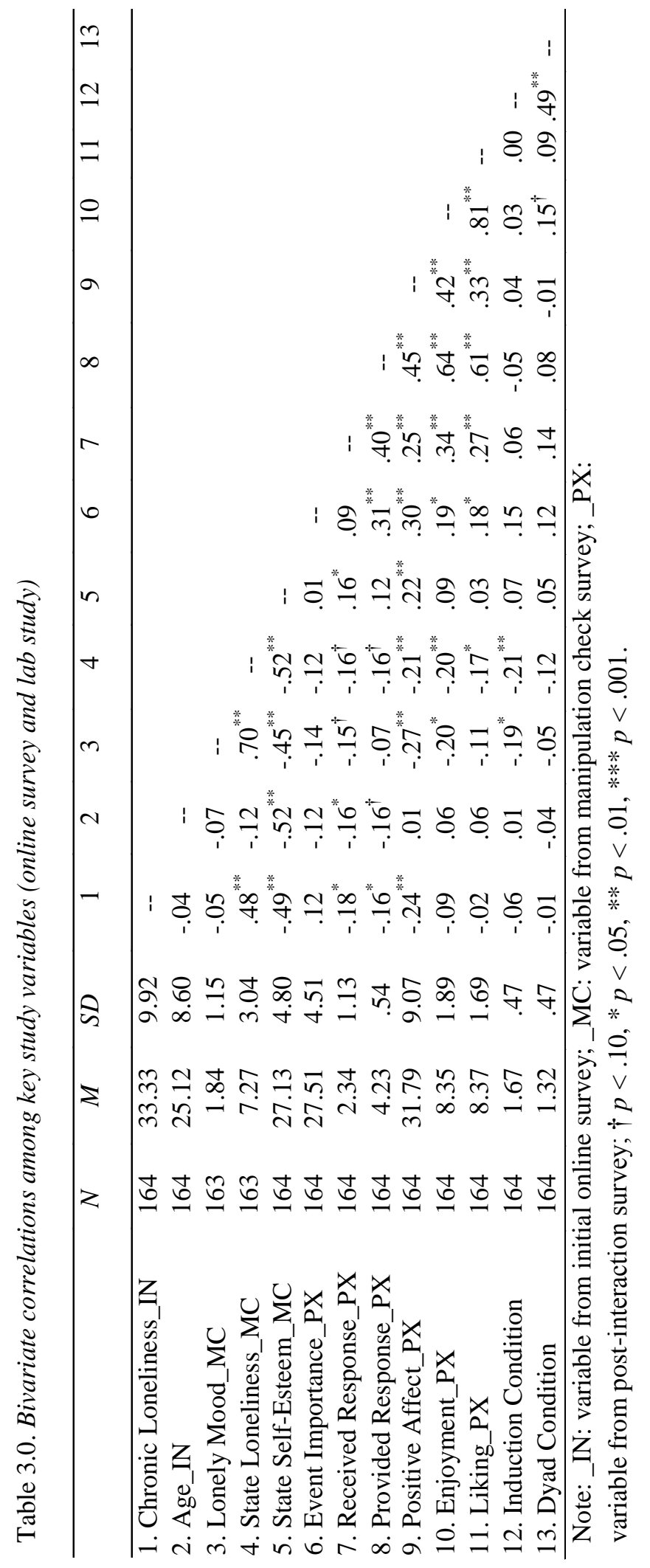




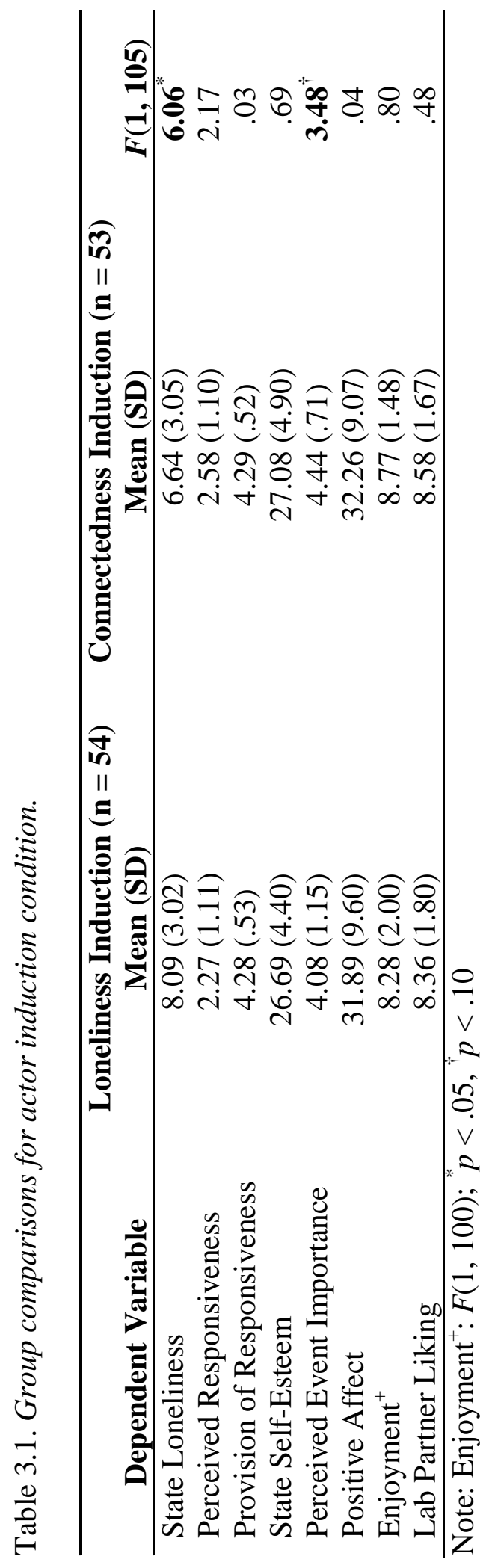




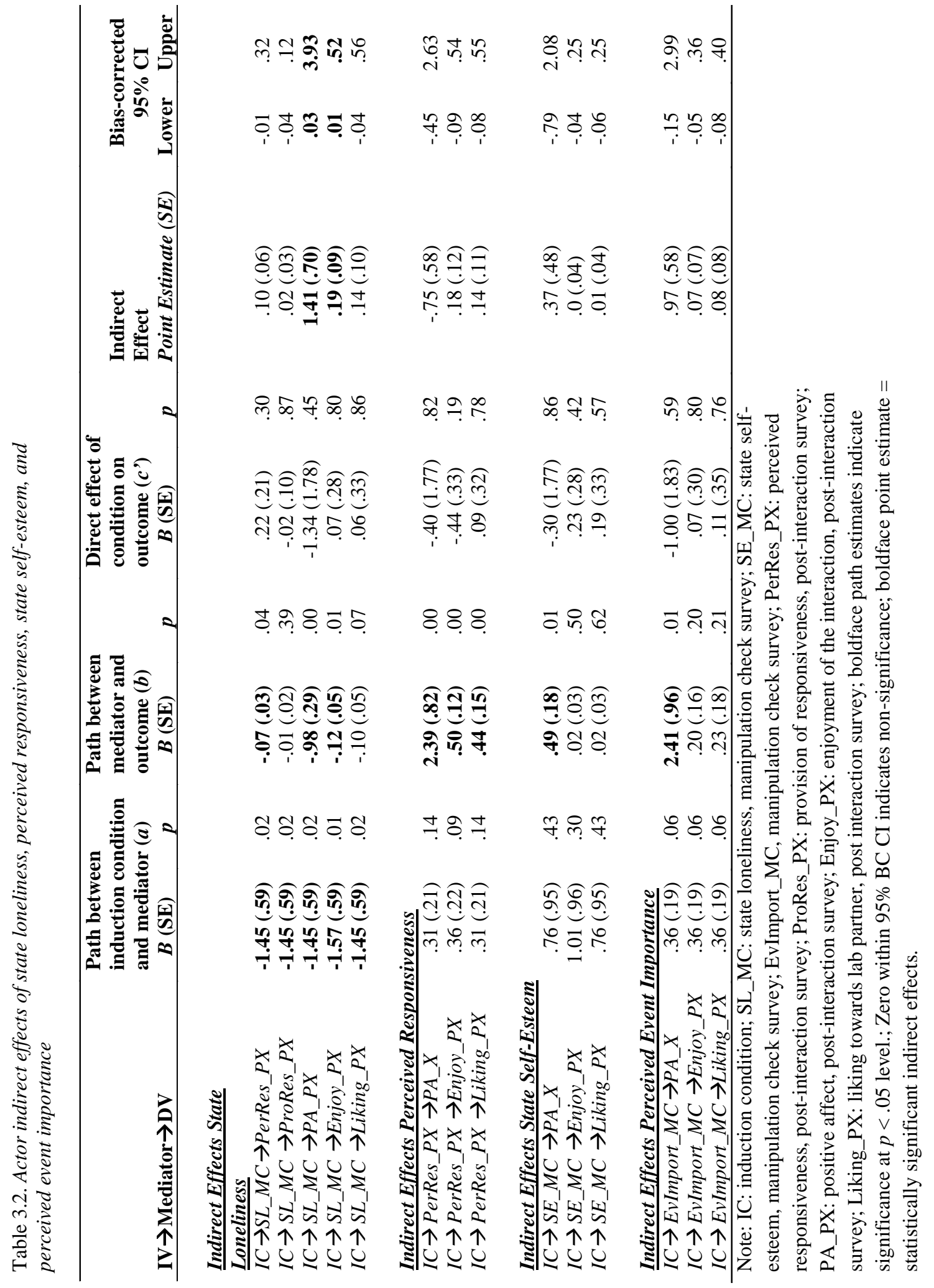


EXPERIMENTAL INDUCTION OF LONELINESS

Table 3.3. Moderated mediation of chronic loneliness on induction effect-state loneliness-positive affect indirect effect.

\begin{tabular}{|c|c|c|c|}
\hline \multicolumn{4}{|c|}{ Positive Affect (Outcome) } \\
\hline Variable & $\boldsymbol{B}$ & SE & $p$ \\
\hline \multicolumn{4}{|c|}{ Mediator Variable Model } \\
\hline Constant & 2.12 & .81 & .01 \\
\hline Induction condition (independent variable) & -1.32 & .51 & .01 \\
\hline Chronic loneliness (moderator) & .03 & .08 & .65 \\
\hline Condition X Chronic Loneliness & .08 & .05 & .10 \\
\hline \multicolumn{4}{|c|}{ Dependent Variable Model } \\
\hline Constant & $\mathbf{3 4 . 3 8}$ & 2.82 & .00 \\
\hline Induction condition (independent variable) & -.96 & 1.78 & .59 \\
\hline Chronic loneliness (moderator) & -.21 & .26 & .44 \\
\hline Condition X Chronic Loneliness & .04 & .17 & .80 \\
\hline State Loneliness (mediator) & -.65 & .34 & .05 \\
\hline State Loneliness X Chronic Loneliness & -.03 & .03 & .33 \\
\hline
\end{tabular}

Conditional Indirect Effects

Levels of Moderator Indirect Effect (SE) Z Bias-Corrected 95\% CI

(Chronic Loneliness) Lower Upper

$\begin{array}{rlrrr}-9.73 & .84(1.08) & .78 & -1.12 & 3.31 \\ .50 & .85(.57) & \mathbf{1 . 4 7} & \mathbf{. 0 3} & \mathbf{2 . 4 7} \\ 10.73 & .39(.76) & .51 & -.73 & 2.49\end{array}$

Note: Boldface path estimates indicate significance at $p<.05$ level; zero within

95\% BC CI indicates non-significance; boldface indirect estimate $=$ statistically significant. 
EXPERIMENTAL INDUCTION OF LONELINESS

Table 3.4. Moderated mediation of chronic loneliness on induction-state loneliness-liking indirect effect.

\begin{tabular}{|c|c|c|c|c|c|}
\hline \multicolumn{6}{|c|}{ Liking Towards Lab Partner (Outcome) } \\
\hline Variable & & B & & SE & $p$ \\
\hline \multicolumn{6}{|c|}{ Mediator Variable Model } \\
\hline Constant & & 2.12 & & .81 & .01 \\
\hline Induction condition (in & lependent variable) & -1.32 & & .51 & .01 \\
\hline Chronic loneliness (mo & derator) & .03 & & .08 & .65 \\
\hline Condition X Chronic L & neliness & .08 & & .05 & .10 \\
\hline \multicolumn{6}{|c|}{$\underline{\text { Dependent Variable Model }}$} \\
\hline Constant & & 8.33 & & .52 & .00 \\
\hline Induction condition (in & lependent variable) & -.03 & & .33 & .93 \\
\hline Chronic loneliness (mo & derator) & .03 & & .05 & .55 \\
\hline Condition X Chronic L & neliness & -.02 & & .03 & .60 \\
\hline State Loneliness (medi & tor) & -.14 & & .06 & .03 \\
\hline State Loneliness X Chr & nic Loneliness & .01 & & .42 & .02 \\
\hline \multicolumn{6}{|c|}{ Conditional Indirect Effects } \\
\hline Levels of Moderator & Indirect Effect $(S E)$ & $\vec{Z}$ & \multirow{2}{*}{\multicolumn{3}{|c|}{$\begin{array}{l}\text { Bias-Corrected } 95 \% \mathrm{CI} \\
\text { Lower }\end{array}$}} \\
\hline (Chronic Loneliness) & & & & & \\
\hline-9.73 & $.55(.27)$ & 2.06 & .14 & & 1.38 \\
\hline .50 & $.17(.11)$ & 1.57 & .01 & & .36 \\
\hline 10.73 & $.01(.06)$ & .09 & -.03 & & .46 \\
\hline
\end{tabular}

Note: Boldface path estimates indicate significance at $p<.05$ level; zero within

$95 \%$ BC CI indicates non-significance; boldface indirect estimate $=$ statistically significant. 
Table 3.5. Moderated mediation of chronic loneliness on induction-state loneliness-enjoyment indirect effect.

\begin{tabular}{|c|c|c|c|}
\hline \multicolumn{4}{|c|}{ Enjoyment of Interaction (Outcome) } \\
\hline Variable & $B$ & SE & $p$ \\
\hline \multicolumn{4}{|c|}{ Mediator Variable Model } \\
\hline Constant & $\overline{2.12}$ & .81 & .01 \\
\hline Induction condition (independent variable) & -1.32 & .51 & .01 \\
\hline Chronic loneliness (moderator) & .03 & .08 & .65 \\
\hline Condition X Chronic Loneliness & .08 & .05 & .10 \\
\hline \multicolumn{4}{|c|}{ Dependent Variable Model } \\
\hline Constant & 8.24 & .53 & .00 \\
\hline Induction condition (independent variable) & .10 & .33 & .76 \\
\hline Chronic loneliness (moderator) & -.01 & .05 & .78 \\
\hline Condition X Chronic Loneliness & .01 & .20 & .85 \\
\hline State Loneliness (mediator) & -.17 & .06 & .01 \\
\hline State Loneliness X Chronic Loneliness & -.05 & .42 & .01 \\
\hline
\end{tabular}

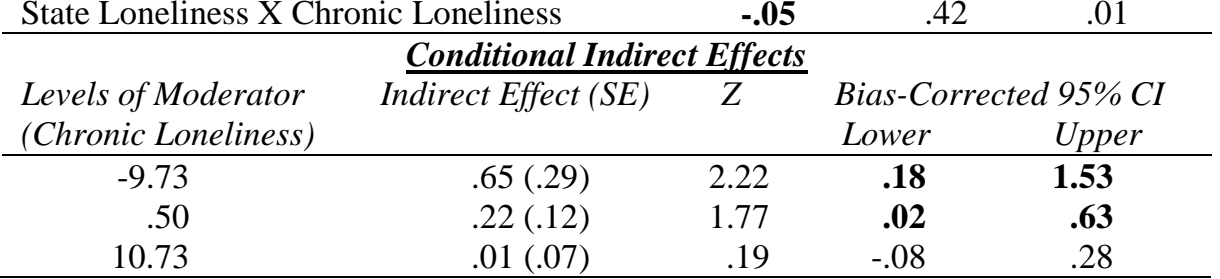

Note: Boldface path estimates indicate significance at $p<.05$ level; zero within

$95 \%$ BC CI indicates non-significance; boldface indirect estimate = statistically significant. 
Table 3.6. Moderated mediation of chronic loneliness on induction condition-perceived responsivenesspositive affect association.

\begin{tabular}{|c|c|c|c|}
\hline \multicolumn{4}{|c|}{ Positive Affect (Outcome) } \\
\hline Variable & $\boldsymbol{B}$ & SE & $p$ \\
\hline \multicolumn{4}{|c|}{ Mediator Variable Model } \\
\hline Constant & 1.95 & .33 & .00 \\
\hline Induction condition (independent variable) & .30 & .21 & .15 \\
\hline Chronic loneliness (moderator) & -.00 & .03 & .88 \\
\hline Condition X Chronic Loneliness & -.02 & .02 & .45 \\
\hline \multicolumn{4}{|c|}{ Dependent Variable Model } \\
\hline Constant & 28.33 & 3.20 & .00 \\
\hline Induction condition (independent variable) & -.38 & 1.75 & .83 \\
\hline Chronic loneliness (moderator) & -.24 & .31 & .45 \\
\hline Condition X Chronic Loneliness & -.02 & .17 & .92 \\
\hline Perceived Responsiveness (mediator) & 1.90 & .84 & .03 \\
\hline $\begin{array}{l}\text { Perceived Responsiveness X Chronic } \\
\text { Loneliness }\end{array}$ & .03 & .09 & .77 \\
\hline
\end{tabular}

\begin{tabular}{|c|c|c|c|c|}
\hline \multirow{3}{*}{$\begin{array}{l}\text { Levels of Moderator } \\
\text { (Chronic Loneliness) }\end{array}$} & \multicolumn{2}{|c|}{ Conditional Indirect Effects } & \multirow{2}{*}{\multicolumn{2}{|c|}{ Bias-Corrected 95\% Cl }} \\
\hline & Indirect Effect $(S E)$ & $\mathrm{Z}$ & & \\
\hline & & & Lower & Upper \\
\hline-9.88 & $.73(.83)$ & .88 & -.23 & 3.23 \\
\hline .38 & $.56(.49)$ & 1.13 & -.14 & 1.85 \\
\hline 10.73 & $.29(.76)$ & .39 & -.88 & 2.49 \\
\hline
\end{tabular}

Note: Boldface path estimates indicate significance at $p<.05$ level; zero within

95\% BC CI indicates non-significance; boldface indirect estimate = statistically significant. 
Table 3.7. Moderated mediation of chronic loneliness on induction condition-perceived responsivenessliking association.

\begin{tabular}{|c|c|c|c|}
\hline \multicolumn{4}{|c|}{ Liking Towards Lab Partner (Outcome) } \\
\hline Variable & $\boldsymbol{B}$ & SE & $p$ \\
\hline \multicolumn{4}{|c|}{ Mediator Variable Model } \\
\hline Constant & 1.95 & .33 & .00 \\
\hline Induction condition (independent variable) & .30 & .21 & .15 \\
\hline Chronic loneliness (moderator) & -.00 & .03 & .88 \\
\hline Condition X Chronic Loneliness & -.02 & .02 & .45 \\
\hline \multicolumn{4}{|c|}{ Dependent Variable Model } \\
\hline Constant & 7.11 & .57 & .00 \\
\hline Induction condition (independent variable) & .06 & .31 & .86 \\
\hline Chronic loneliness (moderator) & .12 & .06 & .04 \\
\hline Condition X Chronic Loneliness & -.02 & .03 & .55 \\
\hline Perceived Responsiveness (mediator) & .48 & .15 & .00 \\
\hline $\begin{array}{l}\text { Perceived Responsiveness X Chronic } \\
\text { Loneliness }\end{array}$ & -.03 & .02 & .04 \\
\hline
\end{tabular}

\begin{tabular}{|c|c|c|c|c|}
\hline \multicolumn{5}{|c|}{ Conditional Indirect Effects } \\
\hline \multirow{3}{*}{$\begin{array}{l}\text { Levels of Moderator } \\
\text { (Chronic } \\
\text { Loneliness) }\end{array}$} & \multirow{3}{*}{$\begin{array}{c}\text { Indirect Effect } \\
(S E)\end{array}$} & \multirow[t]{3}{*}{$Z$} & \multicolumn{2}{|c|}{ Bias-Corrected $95 \% \mathrm{Cl}$} \\
\hline & & & Lower & Upper \\
\hline & & & & \\
\hline-9.88 & $.37(.27)$ & 1.37 & .00 & 1.98 \\
\hline .38 & $.14(.11)$ & 1.24 & -.04 & .42 \\
\hline 10.73 & $.02(.08)$ & .20 & -.09 & .34 \\
\hline
\end{tabular}

Note: Boldface path estimates indicate significance at $p<.05$ level; zero within

$95 \%$ BC CI indicates non-significance; boldface indirect estimate = statistically significant. 
Table 3.8. Moderated mediation of chronic loneliness on induction condition-perceived responsivenessenjoyment association.

\begin{tabular}{|c|c|c|c|}
\hline \multicolumn{4}{|c|}{ Enjoyment of Interaction (Outcome) } \\
\hline Variable & $\boldsymbol{B}$ & SE & $p$ \\
\hline \multicolumn{4}{|c|}{ Mediator Variable Model } \\
\hline Constant & 1.95 & .33 & .00 \\
\hline Induction condition (independent variable) & .30 & .21 & .15 \\
\hline Chronic loneliness (moderator) & -.00 & .03 & .88 \\
\hline Condition X Chronic Loneliness & -.02 & .02 & .45 \\
\hline \multicolumn{4}{|c|}{ Dependent Variable Model } \\
\hline Constant & 6.78 & .61 & .00 \\
\hline Induction condition (independent variable) & .31 & .33 & .36 \\
\hline Chronic loneliness (moderator) & .07 & .06 & .23 \\
\hline Condition X Chronic Loneliness & -.01 & .03 & .82 \\
\hline Perceived Responsiveness (mediator) & .51 & .16 & .00 \\
\hline $\begin{array}{l}\text { Perceived Responsiveness X Chronic } \\
\text { Loneliness }\end{array}$ & -.16 & .45 & .15 \\
\hline
\end{tabular}

\begin{tabular}{|c|c|c|c|c|}
\hline \multirow{3}{*}{$\begin{array}{l}\text { Levels of Moderator } \\
\text { (Chronic } \\
\text { Loneliness) }\end{array}$} & \multicolumn{2}{|c|}{ Conditional Indirect Effects } & \multirow{2}{*}{\multicolumn{2}{|c|}{ Bias-Corrected 95\% CI }} \\
\hline & Indirect Effect & $Z$ & & \\
\hline & $(S E)$ & & Lower & Upper \\
\hline-9.88 & $.34(.26)$ & 1.33 & -.12 & .48 \\
\hline .38 & $.15(.12)$ & 1.24 & -.04 & .43 \\
\hline 10.73 & $.03(.10)$ & .31 & -.09 & .33 \\
\hline
\end{tabular}

Note: Boldface path estimates indicate significance at $p<.05$ level; zero within

95\% BC CI indicates non-significance; boldface indirect estimate = statistically significant. 
EXPERIMENTAL INDUCTION OF LONELINESS

Table 3.9 Group comparisons for dyad condition (interaction partner).

\begin{tabular}{lccc}
\hline \multicolumn{1}{c}{ LONELendent Variable } & $\begin{array}{c}\text { Means (SD) } \\
\text { Mead (n) 57) }\end{array}$ & $\begin{array}{c}\text { CONNECTED Dyad (n = 53) } \\
\text { Means (SD) }\end{array}$ & $\boldsymbol{F ( 1 , 1 0 8 )}$ \\
\hline Perceived Responsiveness & $2.24(1.19)$ & $2.57(1.07)$ & 2.47 \\
Prov. of Responsiveness & $4.14(.55)$ & $4.29(.51)$ & 2.28 \\
Positive Affect & $31.25(8.68)$ & $32.26(9.07)$ & .36 \\
Enjoyment $^{+}$ & $8.03(2.08)$ & $8.87(1.33)$ & $\mathbf{6 . 1 2}^{*}$ \\
Lab Partner Liking & $8.17(1.71)$ & $8.58(1.54)$ & 1.69 \\
\hline
\end{tabular}

Note: Enjoyment ${ }^{+}: F(1,107) ;{ }^{*} p<.05,{ }^{\dagger} p<.10$ 


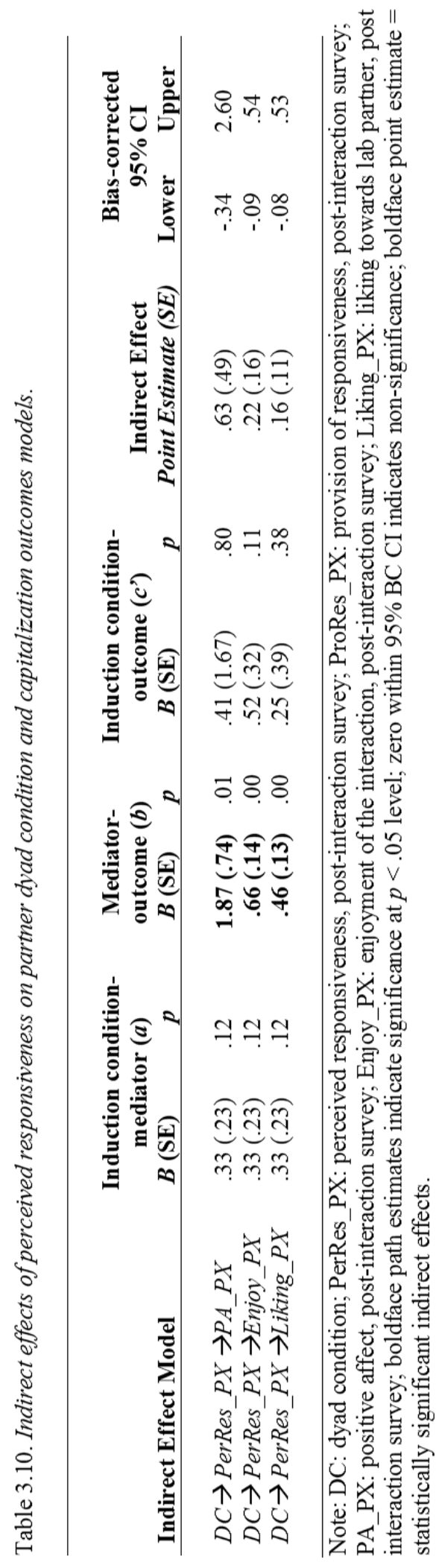


Table 3.11. Summary of hypotheses and results (shaded cells indicate supported hypotheses).

\begin{tabular}{|c|c|c|}
\hline \multicolumn{2}{|c|}{ Hypothesis } & \multirow{2}{*}{\begin{tabular}{|l|} 
Results \\
Supported
\end{tabular}} \\
\hline$H 1.0$ & $\begin{array}{l}\text { Manipulation Check (group differences in state loneliness among induction } \\
\text { groups) } \\
\text { Participants receiving a loneliness induction will report greater levels of } \\
\text { state loneliness following a loneliness induction activity, relative to } \\
\text { participants receiving a social connectedness induction. }\end{array}$ & \\
\hline$H 1.1$ & $\begin{array}{l}\text { 'Actor' induction group differences in perceived responsiveness } \\
\text { 'Lonely' actors will report receiving a less supportive response from their } \\
\text { lab partner, compared to 'connected' actors. }\end{array}$ & Not Supported \\
\hline$H 1.2$ & $\begin{array}{l}\text { 'Actor' induction group differences in provision of responsiveness } \\
\text { 'Lonely' actors will report providing a less supportive response to their lab } \\
\text { partner's event disclosure, compared to 'connected' actors. }\end{array}$ & Not Supported \\
\hline H1.3 & $\begin{array}{l}\text { 'Actor' induction group differences in state self-esteem and perceived event } \\
\text { importance } \\
\text { 'Lonely' actors will report lower levels of state self-esteem following the } \\
\text { mood induction, and will rate their shared positive event as lower in } \\
\text { importance, relative to 'connected' actors. }\end{array}$ & $\begin{array}{l}\text { Partially } \\
\text { Supported } \\
\text { (differences in } \\
\text { perceived event- } \\
\text { importance) }\end{array}$ \\
\hline$H 1.4$ & $\begin{array}{l}\text { 'Actor' induction group differences in post-interaction positive affect, } \\
\text { enjoyment of the interaction, and liking towards lab partner } \\
\text { 'Lonely' actors will report lower levels of positive affect, less enjoyment of } \\
\text { the event-sharing interaction, and less liking towards their lab partner } \\
\text { after the interaction activity, relative to 'connected' actors. }\end{array}$ & Not supported \\
\hline$H 1.5$ & $\begin{array}{l}\text { Indirect effects of state loneliness on actor induction group differences in } \\
\text { perceived responsiveness (received and provided), positive affect, enjoyment } \\
\text { of the interaction, and lab partner liking } \\
\text { The effects of the actor induction condition (lonely vs. social } \\
\text { connectedness) on perceived responsiveness (received and provided), post- } \\
\text { interaction positive affect, enjoyment, and lab partner liking will be } \\
\text { explained by differences in state loneliness. }\end{array}$ & $\begin{array}{l}\text { Partially } \\
\text { Supported } \\
\text { (positive affect } \\
\text { and enjoyment } \\
\text { outcomes) }\end{array}$ \\
\hline$H 1.6$ & $\begin{array}{l}\text { Indirect effects of perceived responsiveness on actor induction group } \\
\text { differences in positive affect, enjoyment of the interaction, and lab partner } \\
\text { liking } \\
\text { The effects of the actor induction condition (lonely vs. social } \\
\text { connectedness) on post-interaction positive affect, enjoyment of the } \\
\text { interaction, and lab partner liking will be explained by differences in } \\
\text { perceived responsiveness. }\end{array}$ & Not supported \\
\hline$H 1.7$ & $\begin{array}{l}\text { Indirect effects of state self-esteem and perceived event importance on actor } \\
\text { induction group differences in positive affect, enjoyment of the interaction, } \\
\text { and lab partner liking } \\
\text { The effects of the actor induction condition (lonely vs. social } \\
\text { connectedness) on post-interaction positive affect, enjoyment of the } \\
\text { interaction, and lab partner liking will be explained by differences in state }\end{array}$ & Not supported \\
\hline
\end{tabular}




\begin{tabular}{|c|c|c|}
\hline & self-esteem and perceived event importance. & \\
\hline$H 1.8$ & $\begin{array}{l}\text { Moderated mediating effects of chronic loneliness on indirect effects of state } \\
\text { loneliness for actor induction group differences in positive affect, enjoyment } \\
\text { of the interaction, and lab partner liking } \\
\text { The indirect effects of state loneliness on actor induction condition- } \\
\text { interaction outcome associations (positive affect, enjoyment, liking) will be } \\
\text { moderated by chronic loneliness. Specifically, individuals at higher levels } \\
\text { of chronic loneliness will exhibit stronger indirect effects. }\end{array}$ & $\begin{array}{l}\text { Partially } \\
\text { supported } \\
\text { (significant } \\
\text { moderated } \\
\text { mediation for all } \\
\text { three outcomes, } \\
\text { but in opposite } \\
\text { direction) }\end{array}$ \\
\hline$H 1.9$ & $\begin{array}{l}\text { Moderated mediating effects of chronic loneliness on indirect effects of } \\
\text { perceived responsiveness for actor induction group differences in positive } \\
\text { affect, enjoyment of the interaction, and lab partner liking } \\
\text { The indirect effects of perceived responsiveness on actor induction } \\
\text { condition-interaction outcome associations (positive affect, enjoyment, } \\
\text { liking) will be moderated by chronic loneliness. Specifically, individuals at } \\
\text { higher levels of chronic loneliness will exhibit stronger indirect effects. }\end{array}$ & Not supported \\
\hline$H 2.1$ & $\begin{array}{l}\text { 'Interaction partner' dyad condition differences in perceived responsiveness } \\
\text { Interaction partners of 'lonely' participants will report less received } \\
\text { responsiveness compared to interaction partners of 'connected' } \\
\text { participants. }\end{array}$ & Not supported \\
\hline$H 2.2$ & $\begin{array}{l}\text { 'Interaction partner' dyad condition differences in the provision of } \\
\text { responsiveness } \\
\text { Interaction partners of 'Ionely' participants will report providing a less } \\
\text { supportive response to their lab partner' event disclosure, compared to } \\
\text { interaction partners of 'connected' participants. }\end{array}$ & Not supported \\
\hline$H 2.3$ & $\begin{array}{l}\text { 'Interaction partner' dyad condition differences in post-interaction positive } \\
\text { affect, enjoyment of the interaction, and lab partner liking } \\
\text { Interaction partners of 'lonely' participants will report less positive affect, } \\
\text { less enjoyment of the interaction, and lower liking towards their lab } \\
\text { partner, compared to interaction partners of 'connected' participants. }\end{array}$ & $\begin{array}{l}\text { Partially } \\
\text { supported (for } \\
\text { enjoyment of the } \\
\text { interaction } \\
\text { outcome) }\end{array}$ \\
\hline$H 2.4$ & $\begin{array}{l}\text { Indirect effects of perceived responsiveness on 'interaction partner' dyad } \\
\text { condition differences in positive affect, enjoyment of the interaction, and lab } \\
\text { partner liking } \\
\text { The effects of partner dyad condition (LONELY vs. CONNECTED) on } \\
\text { positive affect, enjoyment of the interaction, and lab partner liking will be } \\
\text { explained by deficits in perceived responsiveness among interaction } \\
\text { partners of 'lonely' versus 'connected' participants. }\end{array}$ & Not supported \\
\hline
\end{tabular}


Figure 4.0. Indirect effects of state loneliness on the relationship between actor mood induction, perceived responsiveness, self-reported provision of responsive behavior, and capitalization outcome associations (H1.5).

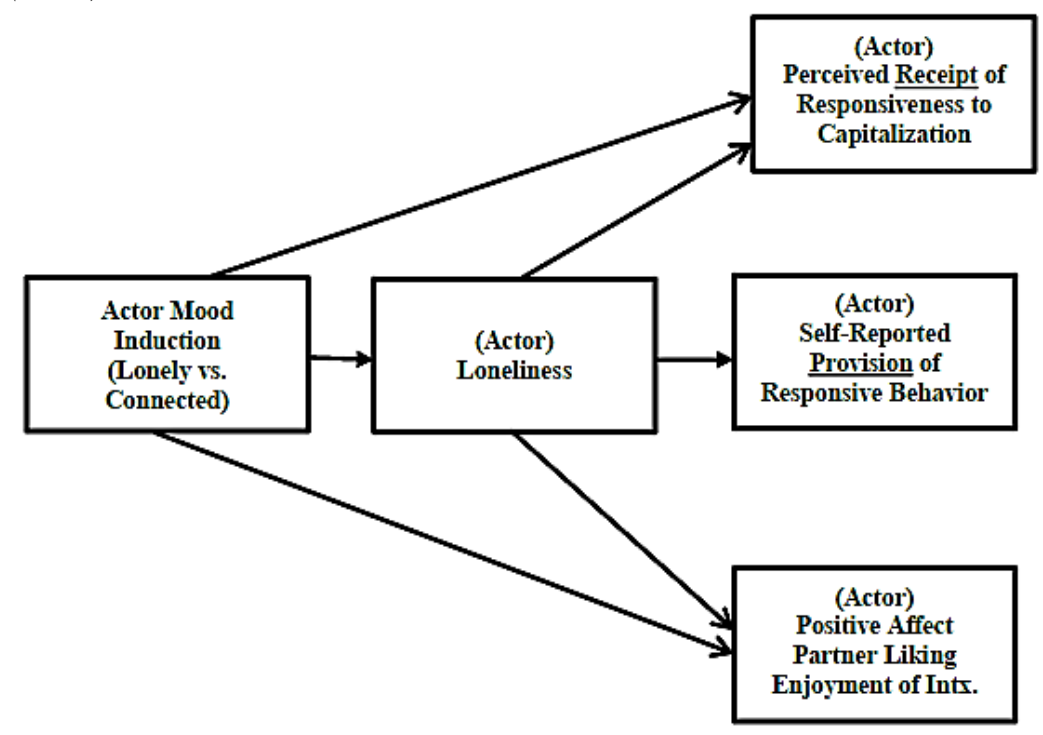


Figure 4.1. Indirect effects of perceived responsiveness on actor mood induction-capitalization outcome associations (H1.6).

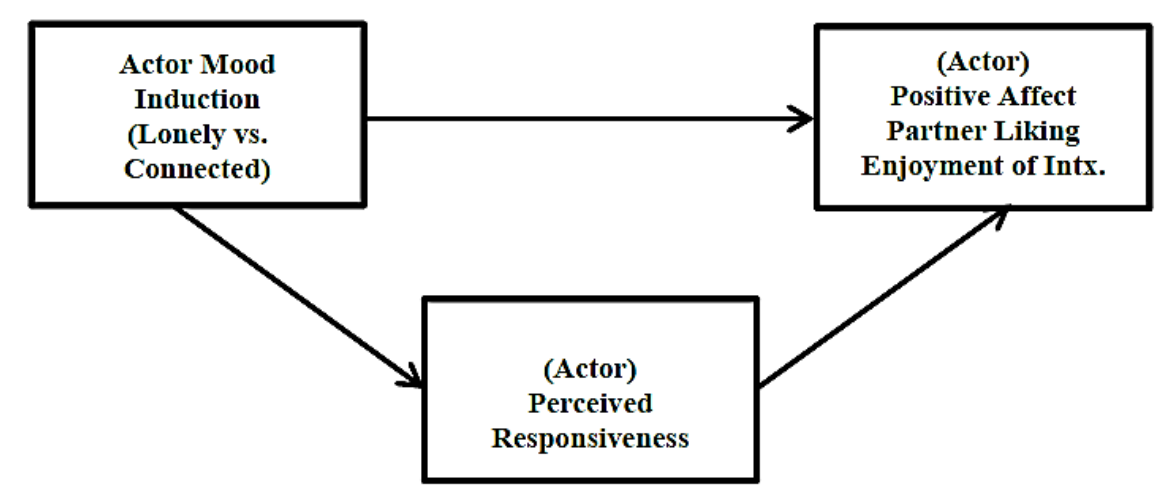


Figure 4.2: Indirect effects of state self-esteem and event importance on the effects of the induction on levels of positive affect, partner liking, and enjoyment of the event-sharing interaction (H1.7)
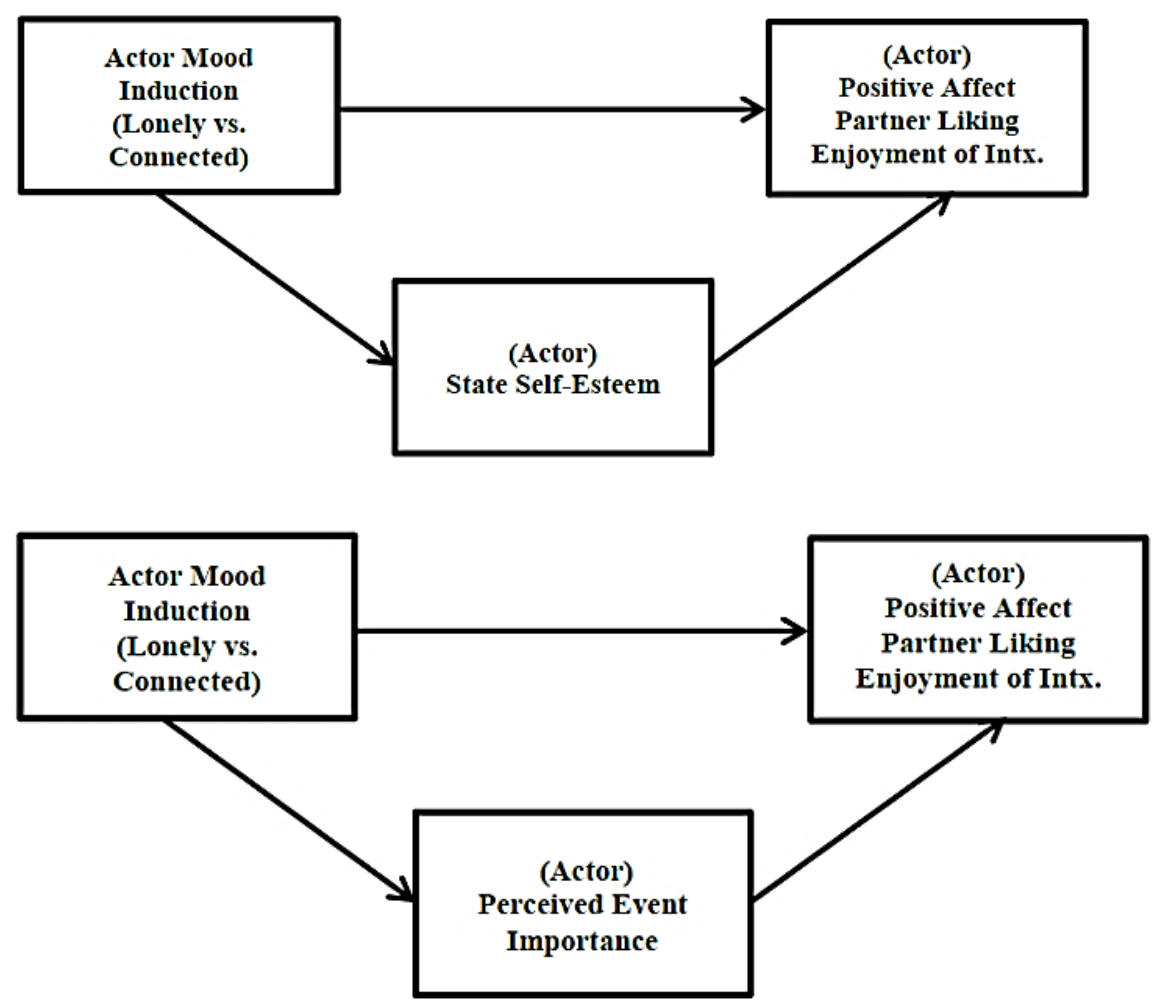
Figure 4.3. Moderation of loneliness on indirect effects of state loneliness (H1.8).

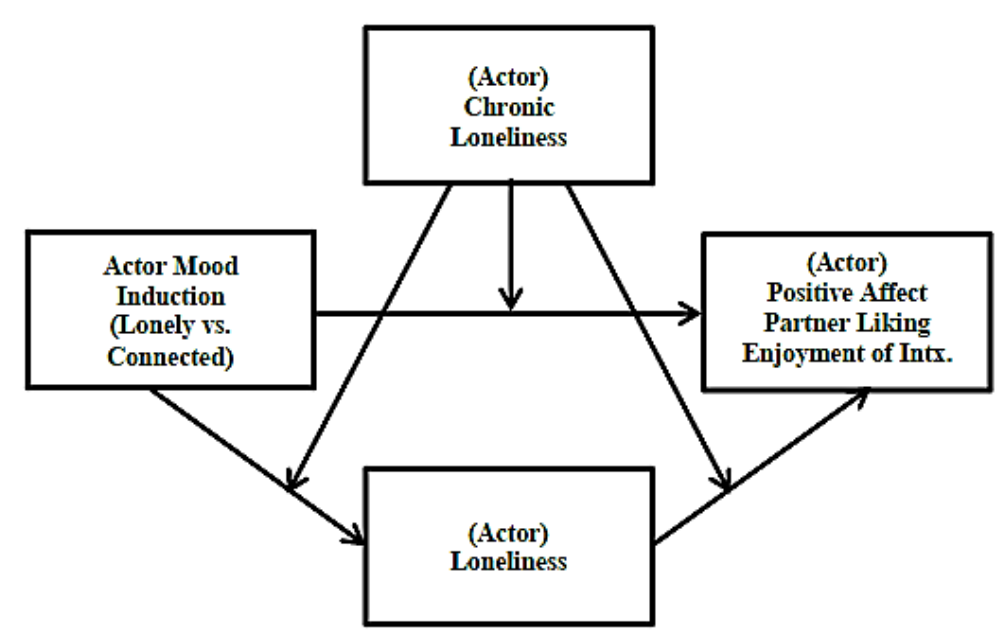


Figure 4.4. Moderation of loneliness on indirect effects of actor perceived responsiveness (H1.9)

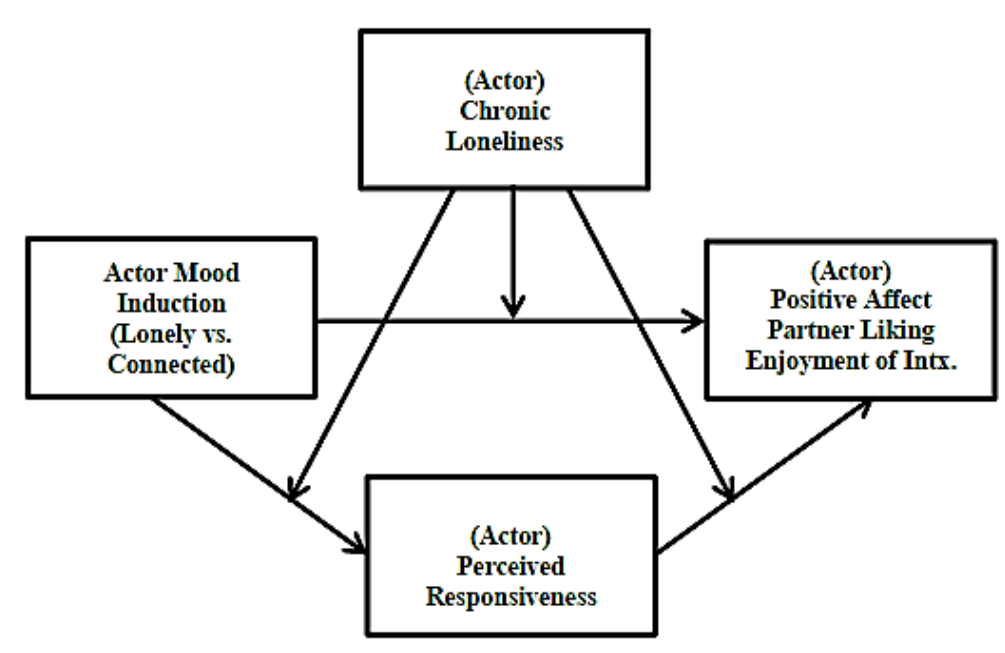


Figure 4.5. Indirect effects of perceived responsiveness on the relationship between partner dyad condition and capitalization outcomes (H2.4).

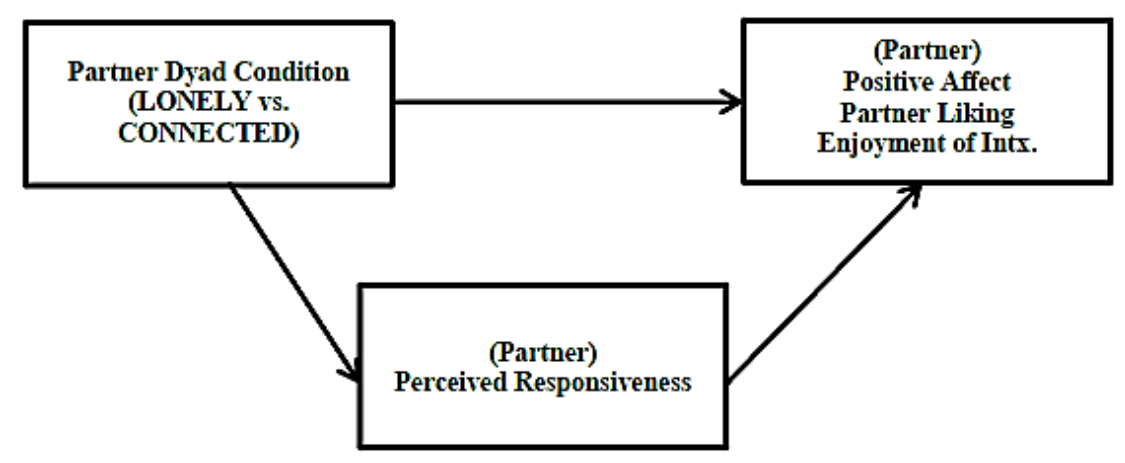


Figure 4.6. Interaction of state loneliness and chronic loneliness on liking towards lab partner.

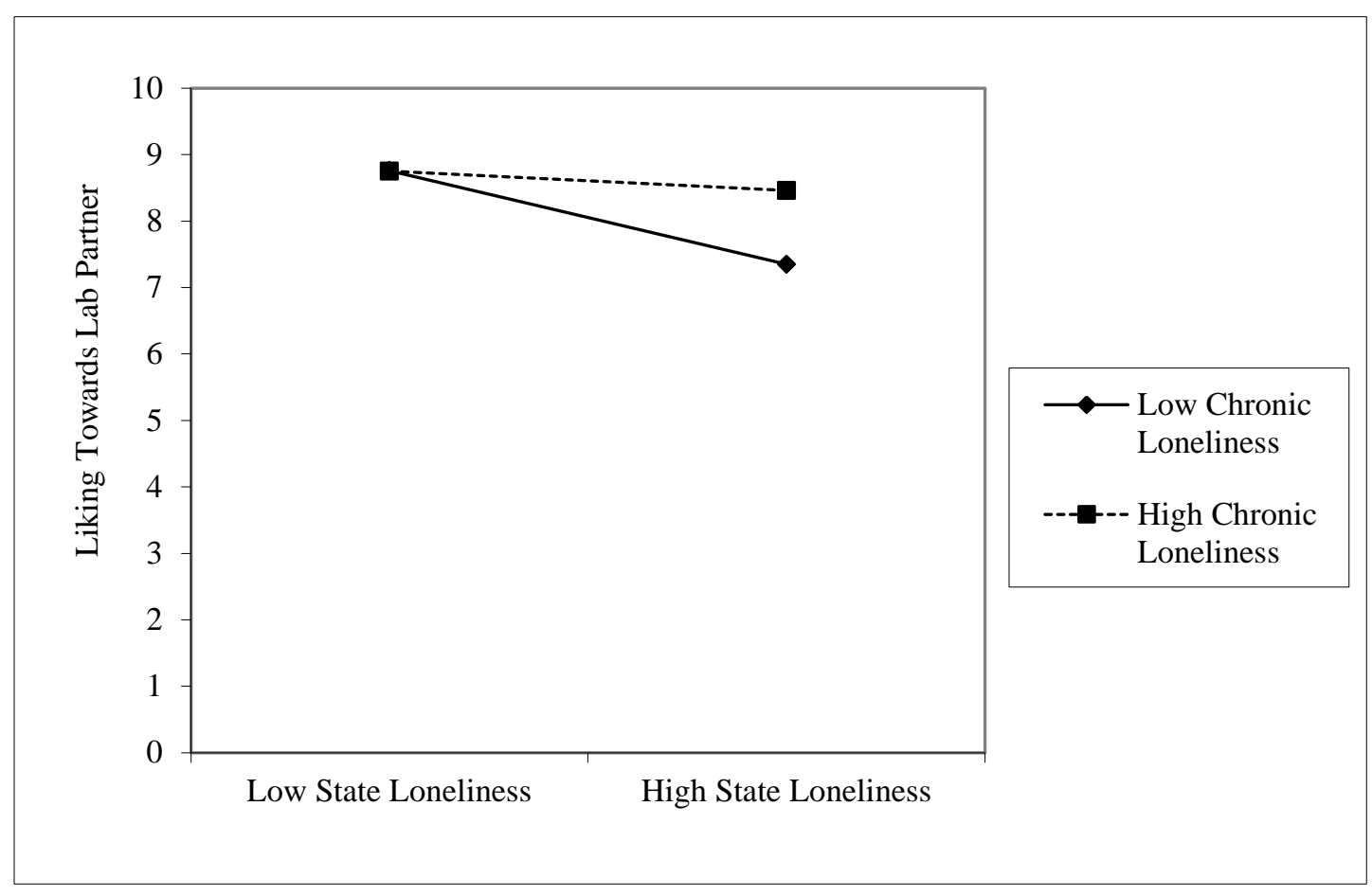


Figure 4.7. Interaction of state loneliness and chronic loneliness on enjoyment of the interaction.

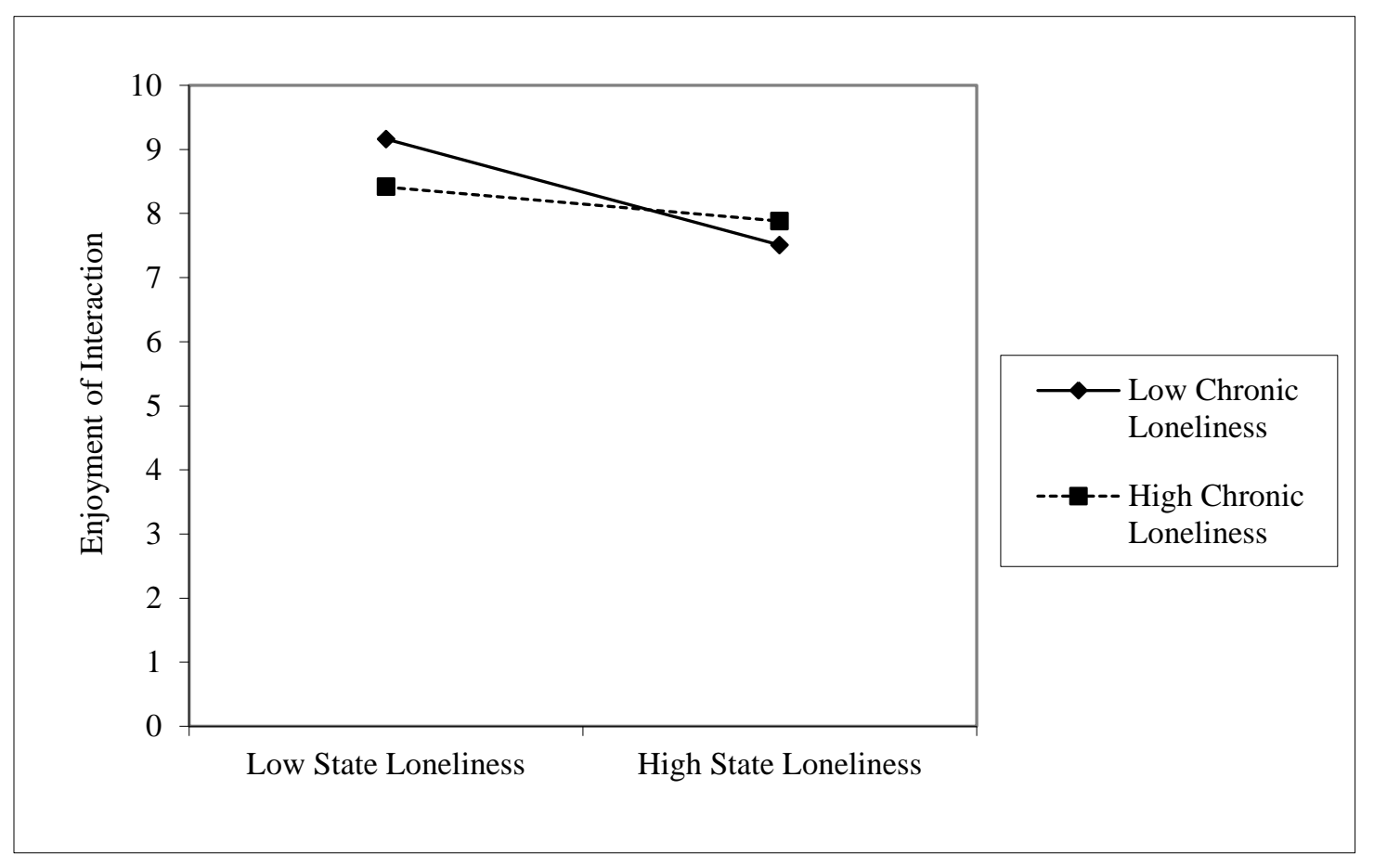


Figure 4.8. Interaction of perceived responsiveness and chronic loneliness on liking towards lab partner.

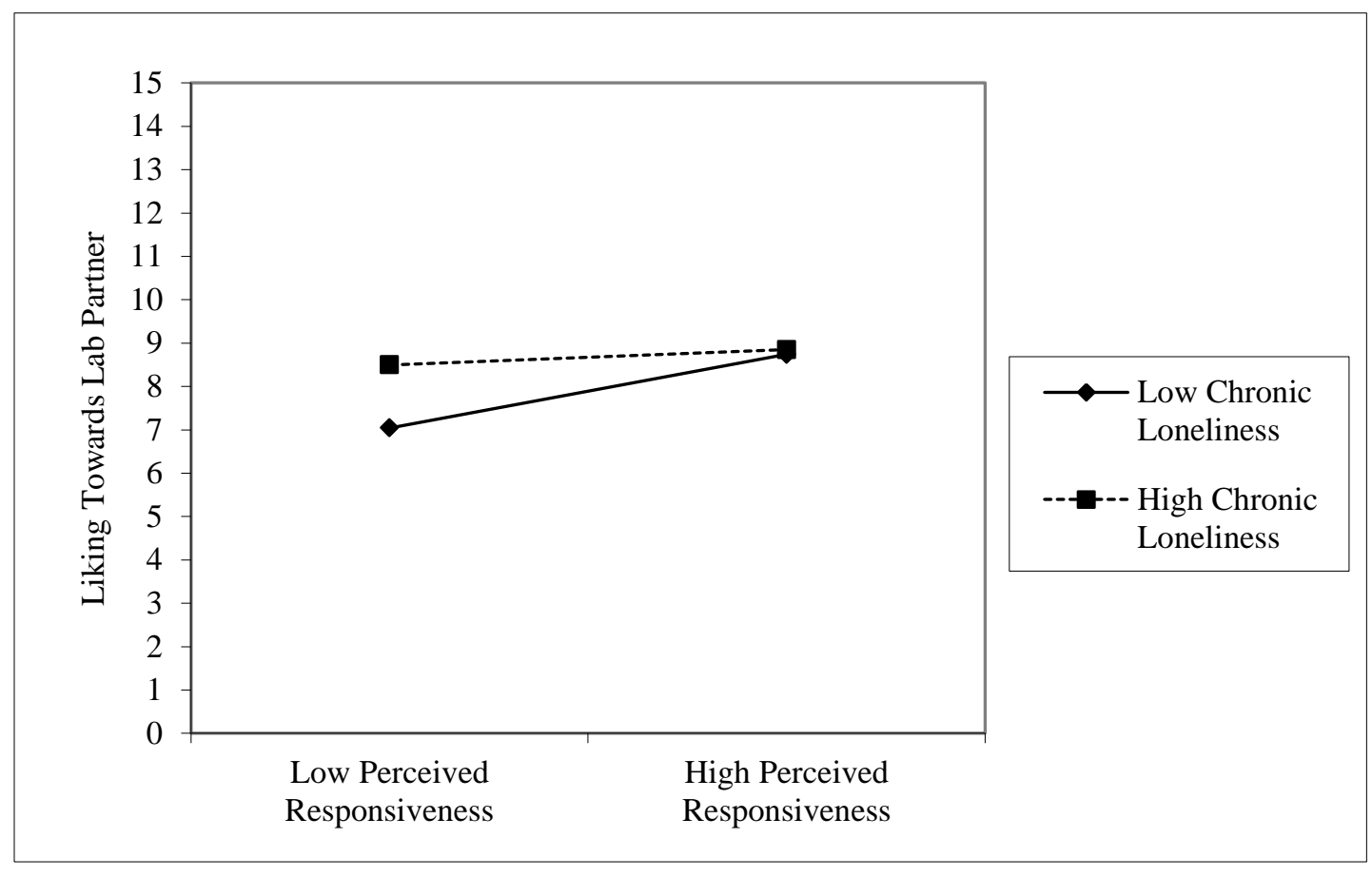




\section{CHAPTER V. DISCUSSION}

This dissertation sought to reveal affective, cognitive, and behavioral correlates of chronic and transient levels of loneliness. Through a series of three studies, the current research provided support for multidimensional and transactional models of loneliness, as well as a positivity-deficit perspective. Additionally, this combination of studies provided valuable insight into the intrapersonal and interpersonal consequences of loneliness, which may negatively impact the development of close relationships and contribute to the maintenance of loneliness over time. Results from the three studies suggest that both chronic and acute experiences of loneliness are consequential to health and social behavior, thus motivating future research to consider both in assessing the short-term and long-term impacts of this negative-affective experience.

\section{Contributions}

Existing theories and models of loneliness have predominantly focused on longlasting or chronic experiences of loneliness. Indeed, some theorists have suggested that transient loneliness is normative and adaptive, whereas chronic levels of loneliness are detrimental to health and well-being over time, and thus should be of central concern to clinical and psychological research (Cacioppo \& Patrick, 2008). Conversely, other theorists have argued that it is imperative to examine processes through which transient loneliness may evolve into a more severe over time (Perlman \& Peplau, 1981). The studies presented in this dissertation took the latter view, providing evidence of the developmental impact of transient loneliness by revealing patterns of behavior (at the within- and between-person level of analysis) which could contribute to negative health 
and social outcomes over time. As such, this dissertation provided a major contribution to the current loneliness literature in relation to the behavioral consequences of transient loneliness. Additionally, through a combination of three studies, this dissertation unpacked unique intrapersonal (i.e., mood-related alcohol consumption) and interpersonal (self-disclosure, and perceived responsiveness) processes related to transient and chronic levels of loneliness, expanding the current understanding of loneliness as consequential to individual health and well-being, to include interpersonal functioning and the development and maintenance of close relationships.

Another major contribution of this dissertation relates to the application of the full-cycle model of social psychology (Mortenson \& Cialdini, 2010), through the use of multiple methods and the triangulation of key findings across multiple studies. In particular, the studies presented in this dissertation applied daily-process methodology and time-lagged multilevel analyses, cross-sectional survey methods, and rigorous experimental methods to examine the various affective, cognitive, and behavioral consequences of loneliness. Previous loneliness research has relied predominantly on cross-sectional and correlational methods. Results presented in this dissertation not only confirmed previously observed associations among loneliness, health, and social behavior, but also provided novel insight into within-person behavioral processes related to transient loneliness which have not previously been captured at the between-person level of analysis (e.g., daily associations among loneliness, time spent with others, and solitary alcohol use). Additionally, the use of experimental, mood-induction methods clarified the directional influence of loneliness on social-perceptual deficits with social 
interactions. Specifically, results from study three demonstrated the causal influence of a loneliness induction on perceptions of event importance, and post-interaction positive affect, enjoyment, and lab-partner liking via increased levels of state loneliness. This was the first experimental study to date to examine the effects of transient loneliness on social perceptions and behaviors within the context of a positive social interaction.

A third major contribution of this dissertation relates to the incorporation of a novel, positivity-deficit perspective of loneliness. Previous research has revealed important information about the various social and behavioral consequences of loneliness, particularly in regards to health risk-related behavior (e.g., alcohol abuse; Heinrich \& Gullone, 2006; Shankur, McMunn, Banks, \& Steptoe, 2011), and stressrelated processes (e.g., negative-event disclosure, support-seeking behavior; Wittenberg \& Reis, 1986). Interestingly, research prior to this dissertation had not explored the extent to which loneliness influences engagement in positive, approach-oriented behavioral processes. As delineated within research and theories of emotion and close relationships, positive and negative behavioral processes are functionally independent, and the presence of negative does not imply the absence of positive (Reis \& Gable, 2013). Thus, examining the influence of loneliness on engagement in positive, approach-oriented behaviors was an important step in understanding this psychological phenomenon.

To address this gap in the current literature, this dissertation builds on recent research by Kashdan, Ferssizidis, Farmer, Adams, and McKnight (2013), who examined 'positivity deficits' related to social anxiety. The current dissertation applied a similar positivity-deficit approach by examining how loneliness interferes with processes vital 
for the maintenance of close, positive interpersonal relationships. Specifically, studies two and three investigated loneliness (chronic and transient) in the context of capitalization, or the disclosure of positive events (Langston, 1994). These were the first studies to date to examine the effects of loneliness on engagement in this positive interpersonal process.

Previous research has described capitalization as vital for the development and maintenance of close relationships. Further, research has demonstrated that sharing positive events with others is related to various intrapersonal and interpersonal benefits (e.g., increased intimacy, lower loneliness; e.g., Gable, Gonzaga, \& Strachman, 2006). The findings presented in studies two and three expand on existing research by showing that levels of loneliness (chronic and transient) prior to a capitalization interaction may actually inhibit individuals from participating in and reaping the personal and social rewards of this positive interpersonal experience. In doing so, these studies make a substantive contribution to the current personal relationships and capitalization literature. Additionally, findings address a recent call by Gable and Reis (2010) for research investigating person factors which interfere with close relationship processes, such as capitalization.

\section{Patterns of Significance}

Though each of the three manuscripts presented in this dissertation provided a unique contribution to the current literature, various patterns of significance were observed among the three studies which are worth noting. These patterns of significance build on previously established multidimensional models of loneliness. Further, results 
across the three studies provide preliminary support for a positivity-deficit perspective of loneliness. Lastly, results suggest that transient loneliness is a unique, powerful, and salient experience for individuals who typically experience low levels of loneliness and/or higher levels of social support.

First, all three studies revealed affective, behavioral, and cognitive deficits related to loneliness which are consistent with previous research and which provide support for multidimensional models (Peplau \& Perlman, 1982). Of note are associations among loneliness, alcohol use, and social-withdrawal observed in study one, loneliness-related deficits in positive event disclosure in study two, and associations among state loneliness, positive affect and enjoyment of a social interaction in study three. Previous work has shown that loneliness is to greater alcohol use (Shankur et al., 2011), social-withdrawal (Cacioppo et al., 2006), less self-disclosure (Wittenberg \& Reis, 1986), less positive affect (Ernst \& Cacioppo, 1999), and more negative perceptions of social interactions (Hawkley, Burleson, Bernston, \& Cacioppo, 2003). Results in both studies one and two are also consistent with theorized responses to loneliness, which include socialwithdrawal and avoidance of social interactions (Rubenstein \& Shaver, 1982).

Additionally, findings of studies one and two are in line with previous research describing loneliness-related deficits in support-seeking behavior (Wilson \& Moulton, 2010). Though spending time with others may serve as an antidote for loneliness, results of study one indicated that rather than seeking out others on days with greater loneliness, participants were withdrawing and drinking. Importantly, this pattern of lonelinessrelated, solitary drinking behavior was observed above and beyond general reductions in 
social behavior. Similarly, whereas seeking support from others in times of good is just as important and beneficial as seeking support in times of stress, results of study two revealed that chronically lonely people were not capitalizing on positive events by celebrating them with others. That loneliness is related to withdrawal from supportive social interactions, in times of good and bad, is one pattern of behavior which may have long term consequences, particularly in regards to the development of close relationships and the maintenance of loneliness over time. As such, results of studies one and two provide very preliminary insight into a developmental process described by transactional models of loneliness (Ernst \& Cacioppo, 1998). Specifically, results suggested that loneliness, at both chronic and transient levels, is related to health and social behavioral deficits, as well as patterns of loneliness-related behavior which may contribute to the exacerbation of loneliness over time. Future research should continue exploring the transactional model of loneliness, through the use of longitudinal methods, which would allow for the examination of specific perceptual and behavioral correlates of loneliness which relate to increased levels and the sustained experience of loneliness over time.

Consistent results were also found across studies two and three in relation to loneliness-related deficits in the perceived importance of positive events. As predicted in study two, chronically lonely persons perceived that their most positive event from the previous week was less important, compared to their less lonely counterparts. Similarly, in study three, participants who received a loneliness induction reported that the positive event they shared with a lab partner during an event-disclosure activity was lower in importance, relative to individuals who received a social connectedness induction. 
Results from both studies are consistent with previously observed patterns of event attributions related to loneliness. That is, previous research has provided evidence that lonely persons are more likely to attribute positive events to external and temporary causes. This pattern of results expands existing research by revealing one particular attributional style and perceptual deficit related to loneliness which may interfere with the development of positive social relationships. Specifically, perceiving positive events as low in importance may influence the extent to which individuals are motivated to capitalize on these events with others. Indeed, and as hypothesized by Kashdan et al. (2013), diminishing the importance of positive events may negatively impact the extent to which individuals celebrate these events with others.

These findings also provide preliminary support for the positive-affect dampening effects of loneliness, which have been previously studied in relation to self-esteem. Of particular note are findings by Wood, Heimpel, and Michela (2003) which showed that individuals low in self-esteem exhibit behaviors and perceptions which dampen experiences of positive affect. Our findings built on this research by suggesting that loneliness may be related to similar affect dampening strategies, via subtle differences in perceptions of importance and causal attributions for positive events made among lonely persons and their less lonely counterparts.

A final pattern of significance relates to the powerful effects of transient loneliness among individuals who typically experience high levels of social support and/or low levels of chronic loneliness. In study one, individuals at higher levels of social support reported stronger associations among daily increases in loneliness and subsequent 
increases in solitary drinking behavior. Similarly, in study three, the indirect effects of the loneliness induction on outcomes of the event-sharing interaction (i.e., positive affect, enjoyment of the interaction, and liking towards lab partner), via levels of state loneliness were only significant among individuals at mean or low levels of chronic loneliness. That is, these findings suggested that, among participants who typically felt socially connected, the loneliness induction related to less positive affect following an eventsharing interaction, less enjoyment of the interaction, and less liking towards a lab partner, due to heightened levels of state loneliness.

Though this pattern of individual differences was unexpected in study three, results provide valuable and preliminary insight into the power of transient loneliness for individuals who typically feel socially connected. Indeed, as described by the cognitivediscrepancy model (Paloutzien \& Janigian, 1987; Perlman \& Peplau, 1981), loneliness is rooted in a perceived discrepancy between what is expected or desired in social relationships and what is experienced in the moment. This discrepancy may be more salient for individuals who generally feel socially connected and supported, as it is in contrast to what is typically experienced within their relationships. That loneliness represents a perceived deficit in social relationships may momentarily diminish the beneficial effects of social support and belongingness, which previous research has demonstrated in relation to negative mood and stress (e.g., Cohen \& Wills, 1985). Thus, this dissertation motivates future work to explore how affective and behavioral reactions to transient loneliness differ based on more prolonged experiences of loneliness and general levels of support. 
CONSEQUENCES OF LONELINESS

\section{Theoretical Implications}

In summary, patterns of significance across the studies presented in this dissertation support previously established multidimensional models of loneliness (Peplau \& Perlman, 1982), which describe loneliness as manifesting in affective, cognitive, and behavioral consequences. Additionally, results provide preliminary support for a positivity-deficit perspective of loneliness by showing that loneliness, when experienced at both chronic and transient levels, is related to deficits in a positive social behavior (i.e., capitalization; Gable et al., 2006). To the extent that positive social interactions, and more specifically capitalization, are vital for the development and maintenance of close relationships, results in this dissertation (i.e., loneliness-related deficits in capitalization) provide insight into one social process which may contribute to the maintenance of loneliness over time.

Importantly, results of this dissertation also challenge and refine recent theorized models of transient loneliness. Specifically, recent theorists have proposed an evolutionary model, wherein transient loneliness is described as normative and adaptive in that it serves as a signal the need to reconnect (Cacioppo \& Patrick, 2008), and activates motives for reafilliation (Qualtner et al., 2015). Conversely, cognitive biases associated with chronic loneliness (e.g., negative perceptions of social interactions, distrust towards others), impede the reafilliation process, and thus chronic loneliness is more detrimental to psychological and physiological health over time (e.g., depression, poor immune-system functioning). The contributions of this dissertation to the evolutionary model of loneliness are two-fold. First, results reveal that transient 
loneliness is related to similar behavioral deficits and cognitive biases as has been previously observed for chronic loneliness (e.g., social withdrawal, negative perceptions of social interactions). In particular, and as demonstrated in Chapter IV of this dissertation, transient loneliness appears to make even positive social interactions less enjoyable and less intrapersonally beneficial (i.e., less positive affect) for individuals and those with whom they interact (Arpin \& Mohr, in preparation). Further, as evidenced in Chapter II, daily experiences of loneliness are associated with risk-related patterns of drinking behavior (i.e., solitary alcohol consumption), previously shown to relate to problematic alcohol use over time (Arpin, Mohr, \& Brannan, 2015). This pattern of results provides strong evidence in support of transient loneliness as consequential in the moment, and relating to outcomes and patterns of behaviors which may indeed be detrimental to individual and interpersonal health and well-being over time.

Second, results presented in this dissertation provide insight into potential mechanisms through which even transient loneliness could interfere with reaffiliation motives, and thus impeded relationship development. That is, to the extent that past interpersonal experiences influence current behavior and the desire for future interaction, as proposed within existing theories of social interaction and self-disclosure (e.g., Baldwin, 1992; Clark \& Lemay, 2010), transient loneliness (and related deficits in positive affect and enjoyment during social interactions) may facilitate negative expectations for future positive social interactions in the initial stages of relationship development. 
As such, the findings in this compilation of studies provide preliminary evidence for the inhibiting effects of transient loneliness on the reaffiliation process, as has been previously observed for chronic loneliness. Research should continue to explore the role of transient loneliness within positive social interactions, as well as the extent to which transient loneliness experienced within one interaction influences the approach versus avoidance of similar (positive) interactions in the future. Such work would provide further insight into social processes through which transient loneliness develops into chronic levels, thus negatively impacting health and well-being over time. Additionally, research should consider individual differences which make some individuals more immune to the negative impacts of transient loneliness. Of note is recent research on social resilience, which describes the capacity of individuals to foster and engage in positive social relationships, and endure in times of social isolation (Cacioppo, Reis, \& Zautra, 2011). Such research would provide insight into potential intervention and prevention approaches which could help individuals (at chronic and transient levels of loneliness) reduce feelings of loneliness in the moment, thus inhibiting the exacerbation of loneliness over time.

More generally, results provide support for need-based models of social relationships (Baumeister \& Leary, 1995; Deci \& Ryan, 2000). As described by the needto-belong hypothesis, when belongingness needs are unmet, individuals experience serious affective, cognitive, and behavioral consequences, including less positive affect, deficits in cognitive ability, and poor self-regulation. To the extent that loneliness is a signal of unmet belongingess needs, this dissertation provided various examples of the 
consequences of the thwarted need for social connection (e.g., social-withdrawal and solitary alcohol use, deficits in self-disclosure behavior, less positive affect, less enjoyment of social interactions).

Interestingly, a component of the need-to-belong hypothesis which is not supported by the current research relates to the promotion of relational value (Mehrabian, 1969). Specifically, early theory suggested that the intrinsic need to belong motivates individuals to engage in behaviors which encourage others to desire mutual affiliation and social interaction. However, findings across the existing literature provide evidence that loneliness is not typically related to behavior which promotes social interaction. Indeed, despite the description of loneliness as a social hunger signaling the need to reconnect (Hawkley \& Cacioppo, 2010), research finds consistent associations among loneliness and subsequent cognitions and behaviors which actually appear to promote withdrawal and isolation, rather than social reaffiliation (e.g., increased vigilance to social threat, negative interpretation of social information, over-attentiveness to social cues; Qualter et al., 2015). The findings of this dissertation provide evidence of similar associations among loneliness and withdrawal-promoting behaviors and cognitions, even in the context of transient or fleeting experiences of loneliness.

\section{Limitations and Future Directions}

Despite the various contributions of this dissertation, questions remain in relation to the social and behavioral consequences of loneliness (chronic and transient) which should be addressed in future research. Additionally, limitations of the studies which make-up the current dissertation motivate future research to continue examining chronic 
and transient loneliness with diverse research samples and more objective assessments of behavior. The section that follows makes note of the limitations of the current dissertation, and describes how these limitations can be addressed in future research.

The first limitation relates to the representativeness of participant samples, particularly in relation to gender. Of note is the gender imbalance in study two and the focus on female dyads in study three. Though the results of studies two and three provide valuable insight into various interpersonal consequences of transient loneliness within positive social interactions, it is possible that the processes explored in these studies differ among men and women. Indeed, researchers posit that, while both men and women are equally prone to loneliness, genders differ in the types of loneliness experienced and in the likelihood of providing accurate self-reports loneliness particularly when using self-labeling measures (e.g., Cacioppo, Gripp, London, Goossens, \& Cacioppo, 2015; Coplan, Closson, \& Arbeau, 2007).

Additionally, early experimental research showed that nonlonely others' perceptions of and reactions to lonely targets differed based on whether the target individual is male or female (Borys \& Perlman, 1985). Recent research has also demonstrated gender differences in responses to transient loneliness (e.g., Arpin, Mohr, \& Brannan, 2015). For example, in study one of this dissertation, women exhibited riskier patterns of daily loneliness-related drinking behavior (i.e., solitary alcohol consumption), relative to men. Previous research has also described gender differences in the provision of social support, with women providing greater responsiveness and support, relative to men (Neff \& Karney, 2005). Additionally, females are typically 
preferred as interaction partners within the context of self-disclosure, given the general tendency to exhibit greater interpersonal warmth within these interactions (Dindia \& Allen, 1992).

In explaining gender differences loneliness and responses to loneliness, researchers and theorists have described the strong interpersonal and communal orientations of women, which may make experiences of loneliness more salient (Wood \& Eagly, 2002). As such, it is possible that the gender imbalance in studies two and three inflated the effects of loneliness on outcomes of interest. Future work should continue to examine gender differences in responses to loneliness (at transient and chronic levels), as well as social-behavioral and perceptual deficits of loneliness within social interactions, within more balanced samples of men and women. In particular, the effects of transient loneliness on perceptions and behaviors within disclosure interactions among men and women would be an important area of research to explore.

Relatedly, the eligibility criteria of studies one and three may have limited the representativeness of these samples in relation to chronic levels of loneliness. Due to ethical concerns relating to the methods used in each study (daily reports of loneliness and alcohol use in study one, and loneliness induction in study three), individuals at risk for depression were not included in these samples. Though loneliness and depression are distinct constructs, the two are highly correlated, and loneliness has previously been described as a major risk factor for depression (Cacioppo et al., 2006). Thus, these screening procedures may have limited these samples to individuals at low to moderate levels of chronic loneliness. Yet, it should be noted that a major contribution of both 
studies one and three is the focus on transient experiences of loneliness. Though previous research has provided much evidence in support of consequences and behavioral correlates of chronic loneliness, these studies were among the first to examine the social behavioral and perceptual consequences of transient loneliness, at the within-person level of analysis and within a lab-based setting. Future work should examine the consequences of transient loneliness among chronically lonely and socially connected individuals alike, who may exhibit unique responses and reactivity to this negative-affective experience.

A final limitation of the studies presented in this dissertation relates to the validity of self-report measures. Importantly, studies one and three offered various methodological strengths which help to address concerns with the use self-report measures. Of note are the multiple daily assessments and time-lagged models employed in study one, which allowed for a more objective assessment of within-person patterns of daily loneliness-related behavior less susceptible to retrospective bias (Reis \& Gable, 2000). Future work should continue investigating transient loneliness via daily-process and experience-sampling research, expanding on the current findings by examining other response behaviors which may be susceptible to self-report and recall bias (e.g., bingeeating behavior).

Additionally, study three reduced participant suspicion by advertising the different components of the study (online survey and lab study) as separate research projects. Yet, surveys completed in the lab were still susceptible to participant response bias, particularly assessments of the perceived receipt and provision of responsiveness during the event-sharing interaction. Future research should seek to build on the findings 
presented in this dissertation by incorporating of more objective measurements of behavior, which are less susceptible to participant response bias. In particular, videos collected in study three should be coded for actual response behaviors exhibited among 'lonely' versus 'connected' participants during the event-sharing interaction. Relatedly, videos from the initial 'getting-to-know-you' activity prior to the mood induction could be coded, and behaviors should be compared for individuals at varying levels of chronic loneliness (as assessed in the initial online survey). Such objective assessments of behavior could offer valuable insights into the social behavioral consequences of chronic and transient loneliness within positive social interactions.

\section{Conclusion}

In summary, this dissertation explores affective, cognitive, and behavioral consequences of loneliness through daily-process, cross-sectional survey, and experimental methods. This combination of studies takes a novel approach by investigating processes through which transient loneliness may develop into chronic levels over time, and by examining loneliness in the context of positive relationship processes and social interactions. Taken together, these studies provide a comprehensive investigation of loneliness, at both the intrapersonal and interpersonal-levels of analysis. Further, this dissertation expands the current understanding of loneliness as consequential for individual health and well-being to include relationship development and interpersonal functioning. 


\section{Terminal Reference List}

Aiken, L. S., \& West, S. G. (1991). Multiple regression: Testing and interpreting interactions. Newbury Park, CA: Sage.

Armeli, S., Mohr, C., Tennen, H., Todd, M., Carney, M. A., Affleck, G., \& Hromi, A. (2003). A daily-process examination of the stress-response dampening effects of alcohol consumption. Psychology of Addictive Behaviors, 17(4), 266-276.

Anderson, C. A., Horowitz, L. M., \& French, R. D. (1983). Attributional-style of lonely and depressed people. Journal of Personality and Social Psychology, 45(1), 127.

Arpin, S. N., \& Mohr, C. D. (in preparation). Experimental induction of loneliness and the perceived provision and receipt of responsive behavior during positive-event disclosure.

Arpin, S. N., \& Mohr, C. D. (in preparation). No one to turn to in times of joy: The inhibiting effects of loneliness on positive-event disclosure.

Arpin, S. N., Mohr, C. D, \& Brannan, D. (2015). Having friends but feeling lonely: A daily-process examination of transient loneliness, drinking behavior, and the influence of interpersonal relationships. Personality and Social Psychology Bulletin, 40, 615-628.

Baldwin, M. W. (1992). Relational schemas and the processing of social information. Psychological Bulletin, 112(3), 461.

Bargh, J. A., \& Chartrand, T. L. (2000). The mind in the middle. Handbook of research methods in social and personality psychology, 253-285. 
Bauer, D. J., Preacher, K. J., \& Gil, K. M. (2006). Conceptualizing and testing random indirect effects and moderated mediation in multilevel models: new procedures and recommendations. Psychological Methods, 11(2), 142.

Baumeister, R. F., \& Leary, M. R. (1995). The need to belong: desire for interpersonal attachments as a fundamental human motivation. Psychological Bulletin, 117(3), 497.

Baumeister, R. F., DeWall, C. N., Ciarocco, N. J., \& Twenge, J. M. (2005). Social exclusion impairs self-regulation. Journal of Personality and Social Psychology, 88(4), 589.

Bekhet, A. K., \& Zauszniewski, J. A. (2012). Mental health of elders in retirement communities: is loneliness a key factor? Archives of Psychiatric Nursing, 26(3), 214-224.

Bell, R. A. (1985). Conversational involvement and loneliness. Communications Monographs, 52(3), 218-235.

Berkman, L. D., \& Breslow, L. (1983). Health and ways of living. New York, New York: Oxford University Press.

Black, D. W., \& Grant, J. E. (2014). DSM-5® Guidebook: The Essential Companion to the Diagnostic and Statistical Manual of Mental Disorders. American Psychiatric Pub.

Bolger, N., Davis, A., \& Rafaeli, E. (2003). Diary methods: Capturing life as it is lived. Annual Review of Psychology 54, 579-616. 
Bolger, N., DeLongis, A., Kessler, R.C., \& Schilling, E. A. (1989). Effects of daily stress on negative mood. Journal of Personality and Social Psychology, 57(5), 808-818.

Bollen, K., \& Lennox, R. (1991). Conventional wisdom on measurement: A structural equation perspective. Psychological Bulletin, 110(2), 305.

Bondevik, M., \& Skogstad, A. (1998). The oldest old, ADL, social network, and loneliness. Western Journal of Nursing Research, 20(3), 325-343.

Bonin, M. F., McCreary, D. R., \& Sadava, S. W. (2000). Problem drinking behavior in two community-based samples of adults: Influence of gender, coping, loneliness, and depression. Journal of Addictive Behaviors, 14(2), 151-161.

Borys, S., \& Perlman, D. (1985). Gender differences in loneliness. Personality and Social Psychology Bulletin, 11(1), 63-74.

Bryk, A. S., \& Raudenbush, S. W. (1992). Hierarchical linear models: Applications and data analysis methods. Newbury Park: Sage.

Burisch, M. (1984). Approaches to personality inventory construction: A comparison of merits. American Psychologist, 39, 214-227.

Burke, M., Marlow, C., \& Lento, T. (2010). Social network activity and social wellbeing. In Proc. CHI 2010: 1902-1912.

Cacioppo J. T., \& Patrick B. (2008). Loneliness: Human Nature and the Need for Social Connection. New York: W. W. Norton \& Company.

Cacioppo, J. T., Ernst, J. M., Burleson, M. H., McClintock, M. K., Malarkey, W. B., Hawkley, L. C., Kowalewski, R. B., Paulsen, A., Hobson, J. A., Hugdahl, K., Spiegel, D., \& Berntson, G. G. (2000). Lonely traits and concomitant 
CONSEQUENCES OF LONELINESS

physiological processes: the MacArthur social neuroscience studies. International Journal of Psychophysiology, 35(2), 143-154.

Cacioppo, J. T., Fowler, J. H., \& Christakis, N. A. (2009). Alone in the crowd: the structure and spread of loneliness in a large social network. Journal of Personality and Social Psychology, 97(6), 977.

Cacioppo, J. T., Hawkley, L. C., \& Berntson, G. G. (2003). The anatomy of loneliness. Current directions in psychological science, 12(3), 71-74.

Cacioppo, J. T., Hawkley, L. C., Crawford, L. E., Ernst, J. M., Burleson, M. H., Kowalewski, R. B., Malarkey, W. B., Van Cauter, E. \& Bernston, G. G. (2002). Loneliness and Health: Potential mechanisms. Psychometric Medicine, 64(407417).

Cacioppo, J. T., Hawkley, L. C., Ernst, J. M., Burleson, M., Berntson, G. G., Nouriani, B., \& Spiegel, D. (2006). Loneliness with a nomological net: An evolutionary perspective. Journal of Research in Personality, 40, 1054-1085.

Cacioppo, J. T., Reis, H. T., \& Zautra, A. J. (2011). Social resilience: the value of social fitness with an application to the military. American Psychologist, 66(1), 43.

Chelune, G. J., Sultan, F. E., \& Williams, C. L. (1980). Loneliness, self-disclosure, and interpersonal effectiveness. Journal of Counseling Psychology, 27(5), 462.

Cacioppo, S., Grippo, A. J., London, S., Goossens, L., \& Cacioppo, J. T. (2015). Loneliness Clinical Import and Interventions. Perspectives on Psychological Science, 10(2), 238-249. 
Clark, M. S., \& Lemay, E. P. (2010). Close relationships. Handbook of Social Psychology.

Clark, M. S., \& Reis, H. T. (1988). Interpersonal processes in close relationships. Annual Review of Psychology, 39(1), 609-672.

Cohen, J. (1988). Statistical power analysis for the behavioral sciences (2 $2^{\text {nd }}$ ed.) Hillsdale, NJ: Erlbaum.

Cohen, S. (1991). Social supports and physical health: Symptoms, health behaviors, and infectious disease. In E. M. Cummings, A. L. Greene, \& K. H. Karraker (Eds.), Life-span developmental psychology: Perspectives on stress and coping (pp. 213233). Hillsdale, New Jersey: Lawrence Erlbaum Associates.

Cohen, S., \& Wills, T. A. (1985). Stress, social support, and the buffering hypothesis, Psychological Bulletin, 98(2), 310.

Cohen, S., Brissette, I., Skoner, D. P., \& Doyle, W. J. (2000). Social integration and health: The case of the common cold. Journal of Social Structure, 1 .

Cohen, S., Mermelstein, R., Kamarck, T., \& Hoberman, H. M. (1985). Measuring the functional components of social support. In I. G. Saraoson \& B. Sarason (Eds.), Social support: Theory, research and applications (pp. 73-94). The Hague, The Netherlands: Martinus Nijhoff.

Collins, N. L., \& Miller, L. C. (1994). Self-disclosure and liking: a meta-analytic review. Psychological Bulletin, 116(3), 457.

Cooper, M. L. (1994). Motivations for alcohol use among adolescents: Development and validation of a four-factor model. Psychological Assessment, 6(2), 117-128. 
Cooper, M. L., Frone, M. R., Russell, M., \& Mudar, P. (1995). Drinking to regulate positive and negative emotions: a motivational model of alcohol use. Journal of Personality and Social Psychology, 69(5), 990.

Coplan, R. J., Closson, L. M., \& Arbeau, K. A. (2007). Gender differences in the behavioral associates of loneliness and social dissatisfaction in kindergarten. Journal of Child Psychology and Psychiatry, 48(10), 988-995.

Cornwell, E. Y., \& Waite, L. J. (2009). Social disconnectedness, perceived isolation, and health among older adults. Journal of Health and Social Behavior, 50(1), 31-48.

Cornwell, E. Y., \& Waite, L. J. (2009). Social disconnectedness, perceived isolation, and health among older adults. Journal of Health and Social Behavior, 50(1), 31-48.

Coxe, S., West, S. G., \& Aiken, L. S. (2009). The analysis of count data: A gentle introduction to Poisson regression and its alternatives. Journal of Personality Assessment, 91(2), 121-136.

Cozby, P. C. (1973). Self-disclosure: a literature review. Psychological bulletin, 79(2), 73, Applied and Preventive Psychology, 8. 1-22.

Creswell, K. G., Chung, T., Clark, D. B., \& Martin, C. S. (2013). Solitary Alcohol Use in Teens Is Associated With Drinking in Response to Negative Affect and Predicts Alcohol Problems in Young Adulthood. Clinical Psychological Science.

Cronbach, L. J. (1951). Coefficient alpha and the internal structure of tests. Psychometrika, 16(3), 297-334.

Cutrona, C. E. (1986). Objective determinants of perceived social support. Journal of Personality and Social Psychology, 50(2), 349-355. 
Davis, S. F., Hanson, H., Edson, R., \& Ziegler, C. (1992). The relationship between optimism-pessimism, loneliness, and level of self-esteem in college students. College Student Journal, 26(2), 244-247.

de Jong-Gierveld, J. (1987). Developing and testing a model of loneliness. Journal of Personality and Social Psychology, 53(1), 119.

Deci, E. L., \& Ryan, R. M. (2000). The "what" and "why” of goal pursuits: Human needs and the self determination of behavior. Psychological Inquiry, 11(4), 227-268.

DeLongis, A., Folkman, S., \& Lazarus, R. S. (1988). The impact of daily stress on health and mood: Psychological and social resources as mediators. Journal of Personality and Social Psychology, 54(3), 486-495.

Dindia, K., \& Allen, M. (1992). Sex differences in self-disclosure: a metaanalysis. Psychological Bulletin, 112(1), 106.

Dindia, K., Fitzpatrick, M. A., \& Kenny, D. A. (1997). Self-Disclosure in Spouse and Stranger Interaction: A Social Relations Analysis. Human Communication Research, 23(3), 388-412.

Durkheim, E. (1857/1951). Suicide. New York: Free Press.

Dykstra, P.A. Fokkema, T. (2007). Social and emotional loneliness among divorced and married men and women: Comparing the deficit and cognitive perspectives. Journal of Applied Social Psychology, 29, 1-12.

Ernst, J. M., \& Cacioppo, J. T. (1998). Lonely hearts: Psychological perspectives on loneliness. Applied and Preventive Psychology, 8. 1-22.

Feldman, S., Downey, G., \& Shaffer-Neitz, R., 1999. Pain, negative mood, and perceived 
support in chronic pain patients: A daily diary study of people with relax sympathic dystrophy syndrome. Journal of Consulting and Clinical Psychology, 67.

Finney, S. J., \& DiStefano, C. (2006). Non-normal and categorical data in structural equation modeling. Structural equation modeling: A second course, 269-314.

Fritz, M. S., \& MacKinnon, D. P. (2007). Required sample size to detect the mediated effect. Psychological Science, 18(3), 233-239.

Gable, S. L., \& Reis, H. T. (2010). Good news! Capitalizing on positive events in an interpersonal context. Advances in Experimental Social Psychology, 42, 195-257.

Gable, S. L., Gonzaga, G. C., \& Strachman, A. (2006). Will you be there for me when things go right? Supportive responses to positive event disclosures. Journal of Personality and Social Psychology, 91(5), 904.

Gable, S. L., Reis, H. T., Impett, E. A., \& Asher, E. R. (2004). What do you do when things go right? The intrapersonal and interpersonal benefits of sharing positive events. Journal of Personality and Social Psychology, 87(2), 228.

Gardner, W. L., Pickett, C. L., Jefferis, V., \& Knowles, M. (2005). On the outside looking in: Loneliness and social monitoring. Personality and Social Psychology Bulletin, 31(11), 1549-1560.

Gerstein, L. H., \& Tesser, A. (1987). Antecedents and responses associated with loneliness. Journal of Social and Personal Relationships, 4(3), 329-363.

Ghiselli, E. E., Campbell, J. P., \& Zedeck, S. (1981). Measurement theory for the behavioral sciences. San Francisco: Freeman. 
Glaser, R., Kiecolt-Glaser, J. K., Speicher, C. E., \& Holliday, J. E. (1985). Stress, loneliness, and changes in herpes virus latency. Journal of Behavioral Medicine, 8(3), 249-260.

Gross, E. F., Juvenon, J., \& Gable, S. L. (2002). Internet use and well-being in adolescence. Journal of Social Issues, 58, 75-90.

Hann, D., Winter, K., \& Jacobsen, P. (1999) Measurement of depressive symptoms in cancer patients. Evaluation of the Center for Epidemiological Studies Depression Scale (CES-D). Journal of Psychosomatic Research, 46, 437-443.

Hawkley, L. C., \& Cacioppo, J. T. (2007). Aging and Loneliness Downhill Quickly? Current Directions in Psychological Science, 16(4), 187-191.

Hawkley, L. C., \& Cacioppo, J. T. (2010). Loneliness matters: a theoretical and empirical review of consequences and mechanisms. Annals of Behavioral Medicine, 40(2), 218-227.

Hawkley, L. C., Burleson, M. H., Bernston, G. G., \& Cacioppo, J. T. (2003). Loneliness in everyday life: Cardiovascular activity, psychosocial context, and health behavior. Journal of Personality and Social Psychology, 85(1), 105-120.

Hawkley, L. C., Hughes, M. E., Waite, L. J., Masi, C. M., Thisted, R. A., \& Cacioppo, J. T. (2008). From social structural factors to perceptions of relationship quality and loneliness: the Chicago health, aging, and social relations study. The Journals of Gerontology Series B: Psychological Sciences and Social Sciences, 63(6), 375384. 
Hawkley, L. C., Masi, C. M., Berry, J. D., \& Cacioppo, J. T. (2006). Loneliness is a unique predictor of age-related differences in systolic blood pressure. Psychology and Aging, 2l(1), 152.

Hayes, A. F. (2009). Beyond Baron and Kenny: Statistical mediation analysis in the new millennium. Communication Monographs, 76(4), 408-420.

Heatherton, T. F. \& Polivy, J. (1991). Development and validation of a scale for measuring state self-esteem. Journal of Personality and Social Psychology, 60, 895-910.

Heatherton, T. F., Wyland, C. L., \& Lopez, S. J. (2003). Assessing self-esteem. Positive Psychological Assessment: A Handbook of Models and Measures, 219-233.

Heinrich, C. C., Brookmeyer, K. A., Shrier, L. A., Shahar, G. (2006). Supportive relationships and sexual risk behavior in adolescence: An ecological-transactional approach. Journal of Pediatric Psychology, 31(3), 286-297.

Heinrich, L. M., \& Gullone, E. (2006). The clinical significance of loneliness: A literature review. Clinical Psychology Review, 26(6), 695-718.

Hofmann, D. \& Gavin, M. (1998). Centering decisions in hierarchical linear models: Implications for research in organizations. Journal of Management, 24(5), 623641.

Holmes, E. A., Mathews, A., Dalgleish, T., \& Mackintosh, B. (2006). Positive interpretation training: Effects of mental imagery versus verbal training on positive mood. Behavior Therapy, 37(3), 237-247. 
House, J. S., Umberson, D., \& Landis, K. R. (1988). Structures and processes of social support. Annual Review of Sociology, 14, 293-318.

Howell, David C. (2010). Statistical Methods for Psychology (7th ed). Belmont, CA: Duxbury Press.

Hussong, A. M., Hicks, R. E., Levy, S. A., \& Curran, P. J. (2001). Specifying the relations between affect and heavy alcohol use among young adults. Journal of Abnormal Psychology, 110(3), 449.

IBM Corp. Released 2013. IBM SPSS Statistics for Windows, Version 22.0. Armonk, NY: IBM Corp.

Ikegami, T. (2002). State self-esteem as a moderator of negative mood effects on person impression. Journal of Experimental Social Psychology, 38(1), 1-13.

Jallais, C., \& Gilet, A. L. (2010). Inducing changes in arousal and valence: Comparison of two mood induction procedures. Behavior research methods,42(1), 318-325

Johnson, J. G., \& Miller, S. M. (1990). Attributional, life-event, and affective predictors of onset of depression, anxiety, and negative attributional style. Cognitive Therapy and Research, 14(4), 417-430.

Jones, W. H., \& Moore, T. L. (1987). Loneliness and social support. Journal of Social Behavior \& Personality, 2(2), 145-156.

Jones, W. H., Hobbs, S. A., \& Hockenbury, D. (1982). Loneliness and social skill deficits. Journal of Personality and Social Psychology, 42(4), 682. 
Joseph, S., \& Lewis, C. A. (1998). The Depression-Happiness Scale: Reliability and validity of a bipolar self-report scale. Journal of Clinical Psychology, 54(4), 537544.

Joseph, S., Linley, P. A., Harwood, J., Lewis, C. A., \& McCollam, P. (2004). Rapid assessment of well-being: The Short Depression-Happiness Scale (SDHS). Psychology and psychotherapy: Theory, research and practice, 77(4), 463-478.

Jourard, S. M. (1964). The transparent self: Self-disclosure and well-being (No. 17). Princeton, NJ: Van Nostrand.

Kashdan, T. B., Ferssizidis, P., Farmer, A. S., Adams, L. M., \& McKnight, P. E. (2013). Failure to capitalize on sharing good news with romantic partners: Exploring positivity deficits of socially anxious people with self-reports, partner-reports, and behavioral observations. Behaviour Research and Therapy, 51, 656-668.

Kashdan, T. B., Week, J. W., \& Savostyanova, A. A. (2011). Whether, how, and when social anxiety shapes positive experiences and events: A self-regulatory framework and treatment implications. Clinical Psychology Review, 31, 786-799.

Kenny, D. A., Kashy, D. A., \& Cook, W. L. (2006). Dyadic data analysis. Guilford Press.

Kenny, D. A., Korchmaros, J. D., \& Bolger, N. (2003). Lower level mediation in multilevel models. Psychological Methods, 8(2), 115. 
Keyes, K. M., \& Hasin, D. S. (2008). Socio-economic status and problem alcohol use: the positive relationship between income and the DSM-IV alcohol abuse diagnosis. Addiction, 103(7), 1120-1130.

Kraut, R., Patterson, M., Lundmark, V., Kiesler, S., Mukophadhyay, T., \& Scherlis, W. (1998). Internet paradox: A social technology that reduces social involvement and psychological well-being? American psychologist, 53(9), 1017.

Kreft, I. G., \& De Leeuw, J. (1998). Introducing Multilevel Modeling. Sage.

LaGuardia, J. G., \& Patrick, H. (2008). Self-determination theory as a fundamental theory of close relationships. Canadian Psychology, 49(3), 201-109.

Langston, C. A. (1994). Capitalizing on and coping with daily-life events: Expressive responses to positive events. Journal of Personality and Social Psychology, 67(6), 1112.

Larsen, R. J., \& Fredrickson, B. L. (1999). Measurement issues in emotion research. In D. Kahneman, E. Diener, \& N. Schwarz (Eds.) Well-being: The foundations of hedonic psychology (pp. 40-60). New York: Russell Sage Foundation.

Lau, S., \& Gruen, G. E. (1992). The social stigma of loneliness: Effect of target person's and perceiver's sex. Personality and Social Psychology Bulletin, 18(2), 182-189.

Laurenceau, J. P., Barrett, L. F., \& Pietromonaco, P. R. (1998). Intimacy as an interpersonal process: the importance of self-disclosure, partner disclosure, and perceived partner responsiveness in interpersonal exchanges. Journal of Personality and Social Psychology, 74(5), 1238. 
Leary, M. R. (2010). Affiliation, acceptance, and belonging: The pursuit of interpersonal connection. In S. T. Fiske, D. T. Gilbert, \& G. Lindzey (Eds.), Handbook of social psychology, $5^{\text {th }}$ edition (Vol. 2, pp. 864-897). Hoboken, NJ: Wiley \& Sons.

Liu, S., Wang, M., Zhan, Y., \& Shi, J. (2009). Daily work stress and alcohol use: testing the cross-level moderation effects of neuroticism and job involvement. Personnel Psychology, 62(3), 575-597.

Maas, C. J., \& Hox, J. J. (2005). Sufficient sample sizes for multilevel modeling. Methodology: European Journal of Research Methods for the Behavioral and Social Sciences, 1(3), 86.

MacGregor, J. C., \& Holmes, J. G. (2011). Rain on My Parade Perceiving Low SelfEsteem in Close Others Hinders Positive Self-Disclosure. Social Psychological and Personality Science, 2(5), 523-530.

MacGregor, J. C., Fitzsimons, G. M., \& Holmes, J. G. (2013). Perceiving low self-esteem in close others impedes capitalization and undermines the relationship. Personal Relationships, 20(4), 690-705.

Maisel, N. C., Gable, S. L., \& Strachman, A. (2008). Responsive behaviors in good times and in bad. Personal Relationships, 15(3), 317-338.

Masi, C. M., Chen, H. Y., Hawkley, L. C., \& Cacioppo, J. T. (2011). A meta-analysis of interventions to reduce loneliness. Personality and Social Psychology Review, $15(3)$. 
Mattick, R. P., \& Clarke, J. C. (1998). Development and validation of measures of social phobia scrutiny fear and social interaction anxiety. Behaviour Research and Therapy, 36(4).

McWhirter, B. T. (1997). Loneliness, learned resourcefulness, and self-esteem in college students. Journal of Counseling and Development, 75(6), 460-469.

Mehrabian, A. (1969). Significance of posture and position in the communication of attitude and status relationships. Psychological Bulletin, 71, 359-372.

Meyers, L. S., Gamst, G., \& Guarino, A. J. (2003). Applied multivariate research. Design and interpretation. Thousand Oaks, CA: Sage Publications, Inc.

Mills, C., \& D'Mello, S. (2013). On the validity of the autobiographical emotional memory task for emotion induction. PloS one, 9(4).

Mohr, C. D., Armeli, S., Ohannessian, C. M., Tennen, H., Carney, A., \& Affleck, G. (2003). Daily interpersonal experiences and distress: Are women more vulnerable? Journal of Social and Clinical Psychology, 22(4), 393-423.

Mohr, C. D., Armeli, S., Tennen H., Temple, M., Todd, M., Clark, J., \& Carney, M. A. (2005). Moving beyond the keg party: A daily-process study of college student drinking motivations. Psychology of Addictive Behaviors, 19(4), 392-403.

Mohr, C. D., Armeli, S., Tennen, H., Carney, M.A., Affleck, G., \& Hromi, A. (2001). Daily interpersonal experiences, context, and alcohol consumption: Crying in your beer and toasting to good times. Journal of Personality and Social Psychology, 80(3), 489-500. 
Mohr, C. D., Brannan, D., Wendt, S., Jacobs, L., Wright, R., \& Wang, M. (2013). Daily mood-drinking slopes as predictors: A new take on drinking motives and related outcomes. Psychology of Addictive Behaviors, 27(4), 944.

Mortensen, C. \& Cialdini, R. (2010). Full-cycle social psychology for theory and application. Social and Personality Psychology Compass, 4(1), 53-3.

Muthén, B. (2011). Applications of causally defined direct and indirect effects in mediation analysis using SEM in Mplus. Unpublished working paper, www.statmodel.com.

Muthén, L. K., \& Muthén, B. O. (2008). Mplus (Version 5.1). Los Angeles, CA: Muthén \& Muthén.

Myers, D. G., \& Diener, E. (1995). Who is happy? Psychological Science, 6(1), 10-19.

Neff, L. A., \& Karney, B. R. (2005). Gender differences in social support: a question of skill or responsiveness?. Journal of Personality and Social Psychology, 88(1), 79.

Ouellet, R., \& Joshi, P. (1986). Loneliness in relation to depression and selfesteem. Psychological Reports, 58(3), 821-822.

Page-Gould, E., Mendoza-Denton, R., \& Tropp, L. R. (2008). With a little help from my cross-group friend: reducing anxiety in intergroup contexts through cross-group friendship. Journal of Personality and Social Psychology, 95(5), 1080.

Paloutzian, R. F., \& Janigian, A. S. (1987). Models and method in loneliness research: Their status and direction. In M. Hogat \& R. Crandall. (Eds). Loneliness: Theory, research, and applications. [Special issue]. Journal of Social Behavior and Personality, 2(2), 31-36. 
Peplau, L. A. (1985). Loneliness research: Basic concepts and findings. In Social support: Theory, research and applications (pp. 269-286). Springer Netherlands.

Peplau, L. A., \& Perlman, D. (1979). Blueprint for a social psychological theory of loneliness. Love and Attraction, 101-110.

Peplau, L. A., \& Perlman, D. (1982). Perspectives on loneliness. Loneliness: A sourcebook of current theory, research and therapy, 1-18.

Peplau, L. A., Miceli, M., \& Morasch, B. (1982). Loneliness and self-evaluation. In L. A. Peplau \& D. Perlman (Eds.), Loneliness: A sourcebook of current theory, research, and therapy (pp. 135-151). New York: Wiley-Interscience,

Perlman, D. (1991). Age differences in loneliness. A meta-analysis. Vancouver, Canada: University of British Columbia. (ERIC Document Reproduction Service No. ED 326767).

Perlman, D., \& Peplau, L. A. (1981). Toward a social psychology of loneliness. Personal Relationships, 3, 31-56.

Perlman, D., \& Peplau, L. A. (1984). Loneliness research: A survey of empirical findings. In L.A. Peplau \& S. Goldston (Eds.), Preventing harmful consequences of severe and persistent loneliness, (pp. 13-46). U.S. Government Printing Office: DDH Publication No. (ADM) 84-1312.

Perrine, M. W., Mundt, J. C., Searles, J. S. \& Lester, L. S. (1995). Validation of daily self-report alcohol consumption using interactive voice response (IVR) technology. Journal of Studies on Alcohol, 56, 487-490. 
Preacher, K. J., \& Hayes, A. F. (2008). Asymptotic and resampling strategies for assessing and comparing indirect effects in multiple mediator models. Behavior Research Methods, 40(3), 879-891.

Pressman, S. D., Cohen, S., Miller, G. E., Barkin, A., Rabin, B. S., \& Treanor, J. J. (2005). Loneliness, social network size, and immune response to influenza vaccination in college freshmen. Health Psychology, 24(3), 297.

Qualter, P., Vanhalst, J., Harris, R., Van Roekel, E., Lodder, G., Bangee, M., Maes, M., Verhagen, M. (2015). Loneliness across the life span. Perspective on Psychological Science, 10, 250- 264.

Raudenbush, S. W., \& Bryk, A. S. (2002). Hierarchical linear models: Applications and data analysis methods. Thousand Oaks, CA: Sage Publications, Inc.

Reis H.T. University of Rochester (2003). A self-report measure of perceived partner responsiveness. Unpublished data.

Reis, H. T., \& Gable, S. L. (2000). Event-sampling and other methods for studying everyday experience. In H. T. Reis \& C. Judd (Eds.), Handbook of Research Methods in Social and Personality Psychology, (pp. 190-222). New York: Cambridge University Press.

Reis, H. T., \& Gable, S. L. (2003). Toward a positive psychology of relationships. In C. L. M. Keyes, J. Haidt (Eds.), Flourishing: Positive psychology and the life welllived (pp. 129-159). Washington, DC, US: American Psychological Association. 
Reis, H. T., Maniaci, M. R., Caprariello, P. A., Eastwick, P. W., \& Finkel, E. J. (2011a). Familiarity does indeed promote attraction in live interaction. Journal of Personality and Social Psychology, 101, 557-570.

Reis, H. T., \& Shaver, P. (1988). Intimacy as an interpersonal process. In S. Duck, E. F.Hay, D.F., S. E. Hobfoll, W. Ickes, B. M. Montgomery (Eds), Handbook of personal relationships: Theory, research and interventions, (pp. 367-389). Oxford, England: John Wiley \& Sons.

Reis, H. T., Clark, M. S., \& Holmes, J. G. (2004). Perceived partner responsiveness as an organizing construct in the study of intimacy and closeness. Handbook of Closeness and Intimacy, 201-225

Reis, H. T., Sheldon, K. M., Gable, S. L., Roscoe, J., Ryan, R. M. (2000). Daily wellbeing: The role of autonomy, competence, and relatedness. Personality and Social Psychology Bulletin, 26(4), 419-435.

Reis, H. T., Smith, S. M., Carmichael, C. L., Caprariello, P. A., Tsai, F. F., Rodrigues, A., \& Maniaci, M. R. (2010). Are you happy for me? How sharing positive events with others provides personal and interpersonal benefits. Journal of Personality and Social Psychology, 99(2), 311.

Resnick, M. D., Beramna, P.S., Blum, R. W., Bauman, K. E., Harris, K. M., Jones, J., Tabor, J., Beuhring, T., Sieving, R. E., Shew, M., Ireland, M., Bearinger, L. H., \& Udry, J. R. (1997). Protecting adolescents from harm: Findings from the national longitudinal study on adolescent health. The Journal of the American Medical Association, 278(10), 823-832. 
Robins, L. N., Cottler, L., Bucholz, K., \& Compton, W. (1995). The Diagnostic Interview Schedule for DSM-IV. Washington University School of Medicine: St. Louis, MO.

Rokach, A., \& Neto, F. (2000). Coping with loneliness in adolescence: A cross-cultural study. Social Behavior and Personality: an international journal, 28(4), 329-341.

Rook, K. S. (1984). The negative side of social interaction: Impact on psychological well-being. Journal of Personality and Social Psychology, 46, 1097-1108.

Rosenberg, M. (1965). The measurement of self-esteem. Society and the adolescent selfimage, 297, 307.

Rotenberg, K. J. (1997). Loneliness and the perception of the exchange of disclosures. Journal of Social and Clinical Psychology, 16(3), 259-276.

Rotenberg, K. J., \& Flood, D. (1999). Loneliness, dysphoria, dietary restraint, and eating behavior. International Journal of Eating Disorders, 25(1), 55-64.

Rotenberg, K. J., \& Kmill, J. (1992). Perceptions of lonely and nonlonely persons as a function of individual differences in loneliness. Journal of Social and Personal Relationships, 9(2), 325-330.

Rotenberg, K. J., Gruman, J. A., \& Ariganello, M. (2002). Behavioral confirmation of the loneliness stereotype. Basic and Applied Social Psychology, 24(2), 81-89.

Rubenstein, C. M., \& Shaver, P. (1982). The experience of loneliness. In L. A. Peplau \& D. Perlman (Eds.), Loneliness: A sourcebook of current theory, research, and therapy (pp. 206-223). New York: Wiley-Interscience. 
Russell, D. W. (1996). UCLA Loneliness Scale (Version 3): Reliability, validity, and factor structure. Journal of Personality Assessment, 66(1), 20-40.

Russell, D., Peplau, L. A., \& Cutrona, C. E. (1980). The revised UCLA loneliness scale: Concurrent and discriminant validity evidence. Journal of Personality and Social Psychology, 39(3), 472-480.

Sarason, B. R., Sarason, I. G., \& Gurung, R. A. R. (2001). Close personal relationships and health outcomes: A key to the role of social support. In B. R. Sarason \& S. Duck (Eds.), Personal relationships: Implications for clinical and community psychology (pp. 15-41). West Sussex, England: Wiley \& Sons, LTD.

Schumaker, J. F., Shea, J. D., Monfries, M. M., \& Groth-Marnat, G. (1993). Loneliness and life satisfaction in Japan and Australia. The Journal of Psychology, 127(1), $65-71$.

Shankur, A., McMunn, A., Banks, J., \& Steptoe, A. (2011). Loneliness, social isolation, and behavioral and biological health indicators in older adults. Health Psychology, 30(4), 377-385.

Sheldon, K. M., \& Gunz, A. (2009). Psychological needs as basic motives, not just experimental requirements. Journal of Personality, 77(5), 1467-1492.

Shrout, P. E., \& Bolger, N. (2002). Mediation in experimental and non-experimental studies: new procedures and recommendations. Psychological Methods, 7(4), 422.

Smith, S. M., \& Reis, H. T. (2012). Perceived responses to capitalization attempts are influenced by self-esteem and relationship threat. Personal Relationships, 19(2), 367-385. 
Sprecher, S., Treger, S., \& Wondra, J. D. (2013). Effects of self-disclosure role on liking, closeness, and other impressions in get-acquainted interactions. Journal of Social and Personal Relationships, 30(4), 497-514.

Solano, C. H., Batten, P. G., \& Parish, E. A. (1982). Loneliness and patterns of selfdisclosure. Journal of Personality and Social Psychology, 43(3), 524.

Steele, C. M., \& Joseph, R. A. (1990). Alcohol myopia: Its prized and dangerous effects. American Psychologist, 921-933.

Strube, M. J. (1991). Small sample failure of random assignment: A further examination. Journal of Consulting and Clinical Psychology, 59(2), 346.

Sweeney, P. D., Anderson, K., \& Bailey, S. (1986). Attributional style in depression: a meta-analytic review. Journal of Personality and Social Psychology, 50(5), 974.

Swendsen, J. D., Tennen, H., Carney, M. A., Affleck, G., Willard, A., \&Hromi, A. (2000). Mood and alcohol consumption: An experience sampling test of the selfmedication hypothesis. Journal of Abnormal Psychology, 109, 198-204.

Tabachnick, B. G., Fidell, L. S., \& Osterlind, S. J. (2001). Using Multivariate Statistics, $5^{\text {th }}$ ed. Boston, MA: Pearson Education, Inc.

Wang, M., Liu, S., Zhan, Y., \& Shi, J. (2010). Daily work-family conflict and alcohol use: Testing the cross-level moderation effects of peer drinking norms and social support. Journal of Applied Psychology, 95(2), 377.

Warburton, W. A., Williams, K. D., \& Cairns, D. R. (2006). When ostracism leads to aggression: The moderating effects of control deprivation. Journal of Experimental Social Psychology, 42(2), 213-220. 
Watson, D., Clark, L. A., \& Tellegen, A. (1988). Development and validation of brief measures of positive and negative affect: The PANAS scales. Journal of Personality and Social Psychology, 54(6), 1063-1070.

Wenger, G.C., Davies, R., Shahtahmasebi, S., \& Scott, A. (1996). Social isolation and loneliness in old age: Review and model refinement. Ageing and Society, 16, $333-358$.

Westermann, R., Spies, K., Stahl, G., \& Hesse, F. W. (1996). Relative effectiveness and validity of mood induction procedures: A meta-analysis. European Journal of Social Psychology, 26(4), 557-580.

Wheeless, L. R., \& Grotz, J. (1977). The measurement of trust and its relationship to selfdisclosure. Human Communication Research, 3(3), 250-257.

Wildschut, T., Sedikides, C., Routledge, C., Arndt, J., \& Cordaro, F. (2010). Nostalgia as a repository of social connectedness: the role of attachment-related avoidance. Journal of Personality and Social Psychology, 98(4), 573.

Wilson, C., \& Moulton, B. (2010). Loneliness among older adults: A national survey of adults 45+. Prepared by Knowledge Networks and Insight Policy Research, Washington, DC: AARP. Nonartists, (45).

Wilson, T. D., Aronson, E., \& Carlsmith, K. (2010). The art of laboratory experimentation. Handbook of Social Psychology.

Wittenberg, M. T., \& Reis, H. T. (1986). Loneliness, social skills, and social perception. Personality and Social Psychology Bulletin, 12(1), 121-130. 
Wood, J. V., Heimpel, S. A., \& Michela, J. L. (2003). Savoring versus dampening: selfesteem differences in regulating positive affect. Journal of Personality and Social Psychology, 85(3), 566.

Wood, W., \& Eagly, A. H. (2002). A cross-cultural analysis of the behavior of women and men: Implications for the origins of sex differences. Psychological Bulletin, 128(5), 699-727.

Young, J. E. (1982). Loneliness, depression, and cognitive therapy: Theory and application. In L. A. Peplau \& D. Perlman (Eds.), Loneliness: A sourcebook of current theory, research, and therapy. New Work, NY: Wiley Inter-science.

Zhou, X., Sedikides, C., Wildschut, T., \& Gao, D. G. (2008). Counteracting Loneliness. On the Restorative Function of Nostalgia. Psychological Science, 19(10), 10231029. 


\section{APPENDIX A: PILOT STUDY PROCEDURE AND RESULTS}

\section{Pilot Study}

\section{Participants}

Sixty-eight undergraduate psychology students ( $86 \%$ female; mean age $=23, \mathrm{SD}$ $=5.48$ ) were recruited for the pilot study via in-class announcements. Psychology instructors provided students with a link to an online study screener, which determined interested participants' eligibility for the study (5-10 minutes). As the pilot procedure involved a negative mood induction procedure, it was deemed unethical to include participants exhibiting risk of depression. Additionally, individuals under the age of 18 were not allowed to participate in the study. Following completion of the study screener, eligible and interested participants were sent the link to an online scheduler where they signed up for a twenty-minute lab session. Participants were sent a reminder for the study two days and two hours before their scheduled lab session. Participants were offered extra credit for participation in the study.

\section{Procedure}

All lab sessions were held in a small office in the psychology department. The room contained a desk with a computer, file cabinet, and a bookshelf. A small flip camcorder positioned on a bookshelf directly across from the participant (participants were told that the camera was used to determine whether they had completed each activity as instructed).

Upon arriving to the lab space, participants were greeted by a research assistant, who administered informed consent and provided instructions throughout the lab session 
for each lab activity. Participants were told that the purpose of the study was to examine different aspects of self-reflection and social perception. During their lab session, participants completed a brief 'self-reflection exercise' (mood induction; 5 minutes), followed by a brief survey (manipulation check; 10 minutes).

Prior to the lab session, participants were randomly assigned and blinded to one of two mood induction conditions: loneliness and social connectedness. Mood induction procedures were based on methods developed by Rotenberg and Flood (1998) and Cacioppo and Hawkley (2006), which utilize the autobiographical recall method. In both conditions, participants were given a small envelope. Within each envelope were two slips of paper, each with a different statement asking participants to think back on a specific type of life experience. The statements within each envelope differed depending on the induction condition. More specific information about the content of each mood manipulation is provided below.

Participants were guided through the self-reflection exercise (mood induction) with a computerized interval timer (Intervals 1 and $2=1.5$ minutes, Interval $3=2$ minutes). An alarm sounded after the end of each time interval. In both loneliness and connectedness mood conditions, participants were told to spend the first two intervals (each 1.5 minutes) taking out one slip of paper at a time, reading it to themselves, and thinking back on a time when they really experienced what was described on each slip of paper. For the third interval, participants were asked to describe these experiences in detail out loud to the video camera. After providing instructions for the 'self-reflection' 
activity (i.e., mood induction), the research assistant turned on the video camera and left the room for the duration of the activity (5 minutes).

\section{Manipulations and Measures}

Loneliness induction. Participants assigned to receive the loneliness induction were given an envelope containing two items adapted from the UCLA loneliness scale. Specific statements included: "Think of a time when you felt you lacked companionship. Perhaps you felt like you had no friends" and "Think of a time when you were no longer close to anyone. Perhaps you felt like you just didn't belong." It is important to note that neither of these items contained the word loneliness, nor were participants directly asked to recall a time when they felt lonely.

Social connectedness induction. Participants receiving the 'connectedness' induction were given two items adapted from methods used by Cacioppo and Hawkley (2006). Specific statements included: "Think of a time when you felt a sense of belonging. Perhaps you were a member of a group" and "Think of a time when you felt you had someone you could share anything with. Perhaps this was a person who was or who could be your best friend."

Post-Induction Survey (Manipulation Check). Immediately following the selfreflection activity, or mood induction exercise, the research assistant re-entered the room and administered a brief computerized survey, designed to assess the effects of the induction on state levels of loneliness and feelings of social connectedness.

State levels of loneliness were assessed as one of the negative affect items on an 18-item version of the Positive and Negative Affect Schedule (PANAS; Watson et al., 
1988). Participants were asked to indicate how lonely they felt since the last interview, based on a 5-point Likert-type scale (0-not at all, 4-extremely). General levels of negative affect were computed by summing the negative affect items on the PANAS. The negative affect subscale showed acceptable internal consistency (Cronbach's alpha $=.76$ ), though fairly low in comparison to previous uses of this measure with college student samples $\left(\right.$ Cronbach's alpha $=.85-.90 ;$ Watson \& Clark, 1999) ${ }^{1}$.

Participants also responded to the belongingness support subscale of the Interpersonal Support Evaluation List (Cohen, Mermelstein, Karmarck, \& Moberman, 1985), which served as a measure of perceived social connectedness. Participants responded to a series of statements probing perceptions of belongingness support availability on a 4-point scale (1-Definitely false, 4-Definitely true). Example items included "When I need suggestions on how to deal with a personal problem, I know someone I can turn to" and "There is someone I can turn to for advice about handling problems with my family. This subscale exhibited adequate reliability (Cronbach's alpha $=.87$ ) comparable to previous research using this measure (e.g., Cohen et al., 1985).

\section{Debriefing}

Upon completing the survey, participants were fully debriefed. As a part of the debriefing process, participants completed a brief self-affirmation exercise to ensure that the use of deception and mood induction procedures in the study did not have negative effects on participant well-being following study participation. Participants were asked to spend 1-2 minutes writing down the most positive aspects of themselves on a notecard, which they were then instructed to take home with them. Following the completion of 
this exercise, participants were thanked for their participation and provided with a list of on-campus mental health resources and the investigator contact information.

\section{Data Analysis Strategy}

To assess the effects of the mood induction on levels of lonely mood (vs. social connectedness), a one-way analysis of variance was conducted comparing participant reports of lonely mood and feelings of belongingness for lonely and connectedness induction conditions. Additionally, to examine the effects of the mood induction on lonely mood over and above general levels of negative affect, a multiple regression was conducted wherein induction condition predicted levels of lonely mood, controlling for general negative affect. Gender differences in the induction effect on levels of lonely mood were also examined as an exploratory step via a 2 (male, female) X 2 (loneliness induction, connectedness induction) factorial ANOVA.

\section{Data Screening}

As suggested by Mills and D’Mello (2014), outliers on the lonely mood variable were identified by computing z-scores of the emotion ratings (i.e., computing z-scores). Given the small sample size, cases with z-scores greater than the absolute value of 2.5 were eliminated from all analyses. Using this method, two emotion ratings were identified as outliers and removed from the data. Additional data from two participants was eliminated due to non-compliance with the self-reflection exercise, as indicated by video footage of the self-reflection activity. Data screening procedures rendered a final pilot sample size of $\mathrm{N}=63$ (lonely induction condition, $\mathrm{n}=33$; connectedness induction, $\mathrm{n}=30$ ). 


\section{Results}

Preliminary descriptive statistics revealed that sixty-five percent of the pilot participants were employed, and $81 \%$ made less than $\$ 16,000$ a year. Sixty-eight percent of participants were non-Hispanic/White, 12\% Hispanic/Latino, 5\% African American, 14\% Asian American, 6\% Native American or Alaskan, 2\% Native Hawaiian, $6 \%$ identified as 'Other'. Sixty percent of participants were in a romantic relationship.

Results of a one-way analysis of variance revealed that induction conditions significantly differed in levels of lonely $\operatorname{mood}\left(F(1,61)=5.51, p=.02, \eta^{2}=.08\right)$. Specifically, participants in the loneliness induction condition reported significantly higher levels of lonely mood following the self-reflection exercise $(M=1.27, S D=1.04)$ compared to participants in the social connectedness induction condition $(M=.70, S D=$ .99). Interestingly, there were no significant differences in feelings of social connectedness between the two induction groups $(F(1,61)=.54, p=.46)$, as assessed by the belongingess subscale of the Interpersonal Support Evaluation List (Cohen et al., $1985)^{2}$. However exploratory analyses revealed marginally significant differences between the two groups in levels of positive affect $\left(F(1,61)=3.12, p=0.08, \eta^{2}=.05\right)$, such that participants in the social connectedness induction condition reported higher levels of positive mood $(M=12.93, S D=5.76)$ following the self-reflection exercise compared to participants in the loneliness mood induction condition $(M=10.48, S D=$ 5.24).

Exploratory analyses revealed significant differences in general levels of negative affect between the two induction groups $\left(F(1,61)=4.28, p=.04, \eta^{2}=.06\right)$. As would be 
anticipated, participants receiving the loneliness induction reported higher levels of negative $\operatorname{mood}(M=9.94, S D=2.47)$, relative to participants who received the connectedness mood induction $(M=8.52, S D=2.93)$. To determine whether the mood induction influenced levels of loneliness, above and beyond increases in negative affect, an analysis covariance was conducted, controlling for levels of general negative affect. Results revealed marginally significant differences in lonely mood between induction groups, when controlling for general negative affect $\left(F(1,60)=3.01, p=.09, \eta^{2}=.05\right)$.

Results of a 2X2 factorial ANOVA revealed no gender differences in lonely mood following the induction $(F(1,61)=.82, p=.37)$. Additionally, there were no differences in the effects of the induction on levels of lonely mood among men and women $(F(1,61)$ $=1.65, p=.20)$.

\section{Brief Discussion}

Results of the pilot study provided support for the effectiveness of the loneliness versus connectedness mood induction procedure in influencing levels of lonely mood. Specifically, participants who reflected and discussed experiences relating to a time when they felt they did not belong reported higher levels of lonely mood in a post-induction mood assessment, relative to participants who reflected on and discussed experiences when they felt a sense of belongingness. Interestingly, induction conditions did not differ in levels of social connectedness following the induction exercise. This was likely due to the measure of perceived belongingness/connectedness included in the pilot study, which was originally developed as an assessment of more stable perceptions of support. The 
results of the pilot demonstrated the need to include a more state-level measure of connectedness in the primary study.

Results of the pilot revealed that the effects of the loneliness induction were reduced to marginal significance when controlling for general negative affect. However this attenuation would be expected given the strong correlation between lonely mood and negative affect $(r=.39, p=.002)$. Loneliness itself is a negative emotional experience, associated with increases in other forms of negative affect (e.g., sadness, boredom, anxiety; Perlman \& Peplau, 1984). Further, that the effects of the induction on lonely $\operatorname{mood}\left(\eta^{2}=.08\right)$ were larger than those observed for general negative affect $\left(\eta^{2}=.06\right)$ supports the use of the induction procedure as an effective method for inducing feelings of loneliness, beyond general levels of negative affect. However, given these results, it was determined necessary to control for negative affect when testing the effects of the induction procedure in the primary study.

Lastly, results of the pilot study indicated that there were no gender differences in the effect of the induction on levels of lonely mood. However, these results should be interpreted with caution, given the gender imbalance in the pilot sample ( $86 \%$ female), and potential for limited power in the test of interaction effects, due to the small overall sample size $(\mathrm{N}=68)$. Despite the absence of gender differences in induction effects as demonstrated in the pilot study, it was determined necessary to fix gender in the primary study. As the primary study included an in-lab interaction among two participants, it was anticipated that focusing on same gender dyads would allow for greater experimental 
control, particularly in regards to gender differences in communication-style, self-

disclosure behavior, and social support provision (e.g., Dindia \& Allen, 1992).

\section{Footnotes}

${ }^{1}$ Though the items on the negative affect subscale of the PANAS showed acceptable internal consistency (Cronbach's alpha $=.76$ ), there was concern with the reliability of this measure, compared to other published studies using a 20-item version of the PANAS, which typically report a much higher alpha for the negative affect subscale (Cronbach's alpha $=.85-.90)$. Thus, the original 20-item version of the PANAS for the primary study, which has previously demonstrated high reliability when administered to college student samples, was utilized in the primary study (Watson, Clark, \& Tellegen, 1999).

${ }^{2}$ Following the completion of the pilot study, it was determined that a different measure of perceived belongingness should be included in the manipulation check survey for the primary study. As the support measure included in the pilot post-induction survey was the subscale of a larger social support measure which is typically used to assess more stable perceptions of support, it is likely that this measure did not capture more state-like perceptions of social connectedness influenced by the connectedness mood induction. 
APPENDIX B: LAB PROCEDURE

Figure 4.9. Overview of Experimental Lab Procedure

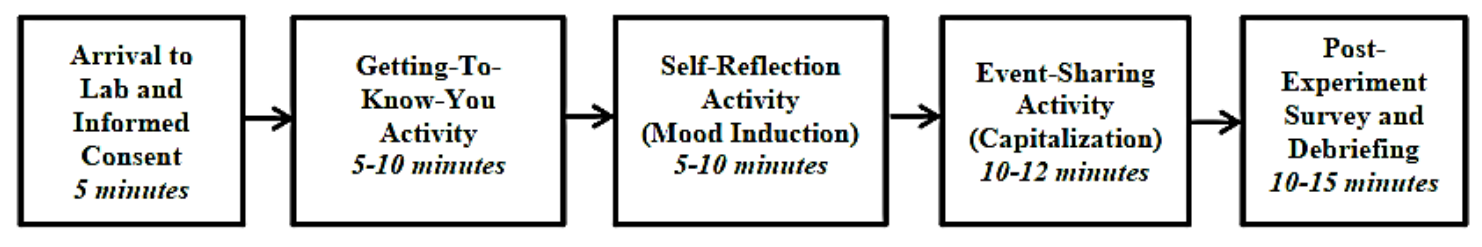

\section{$\underline{\text { LAB SCRIPT }}$}

\section{Getting-To-Know-You Activity}

Hello, and welcome to the study of social exchange and interpersonal perception. Today, you will be asked to complete three brief activities and two brief surveys. Some of these activities you will be completing together, and some by yourself. We will guide you through each activity.

For the first activity, we want you to spend a bit of time getting to know each other. As described in your informed consent, although all aspects of the interaction will remain confidential, we will be recording this exercise to make sure it has been completed as instructed. Please spend the next five minutes switching back and for answering the questions on this list. These are just questions which will help you get to know one another, such as year in school, major, favorite color, etc. I will set a timer, leave the room, and start the camera. I will re-enter after five minutes and let you know when time is up.

Any questions?

Go ahead and begin...

\section{Example getting-to-know-you questions}

1. What is your name?

2. What is your major?

3. What is your favorite color?

4. How old are you?

5. What is your favorite thing to do?

6. What is your favorite food?

7. What is your favorite book?

8. Do you like to sing?

9. Do you like to dance?

10. Do you like to play a musical instrument? 
11. What musical instruments can you play?

12. What is your favorite type of art?

13. Have you ever seen a Broadway musical? Do you like theater?

14. What do you want to be doing in five years? Ten years?

15 . What was your favorite class last year?

16. When you have an hour of free time, what do you like to do?

[After the five-minute getting-to-know-you exercise, ask Participant A (as indicated on the lab roster) to stay in interaction room, and lead other participant to computer room.] Self-Reflection Exercise (Experimental Mood Induction)

For this part of the study, you will be completing a brief self-reflection exercise. In this envelope are two slips of paper. Each slip of paper contains a statement describing a specific type of life experience. You will be guided through this activity with the following interval timer.

[Point to interval timer which should already be pulled up and maximized on computer screen.]

As you can see, there are three intervals. The first two are each a minute and a half, and the third is two minutes long. For the first two intervals, I ask that you take each slip of paper one at a time, read it to yourself, and spend time thinking back on a time when you really felt what is described on each slip of paper. So, for example, when you start the timer take out one slip of paper, read it, and think to yourself about a time when you felt what it describes. A timer will go off after each interval, and so after the first timer goes off, take out the next slip of paper, read it to yourself, and again think back on that experience for the next minute and a half. When the second timer goes off, indicating the beginning of the third interval, you need to start describing these experiences out loud for two minutes. A final alarm will alert you the end of the activity.

Any questions? [Give participant envelope and say]:

I will be waiting outside with my own timer and will reenter the room after five minutes. You may begin. [Specific items in envelopes for each condition listed below]

\section{Loneliness:}

Think of a time when you felt you lacked companionship. Perhaps you felt like you had no friends.

Think of a time when you were no longer close to anyone - Perhaps you felt like you just didn't belong. 


\section{Social Connectedness:}

Think of a time in which you felt a sense of belonging. Perhaps you were a member of a group.

Think of a time when you felt you had someone you could share anything with. Perhaps this was a person who was or who could be your best friend.

[At the end of five minutes, research assistant re-enters the room and administers the manipulation check survey]:

The next part of the study is a brief survey. Please read all of the instructions and respond as accurately as possible. When you are finished, come and alert a research assistant.

\section{Event-Sharing Activity (Capitalization Interaction)}

In this next activity, we are interested in how people discuss their positive events and experiences with others. As in the first activity you completed with your partner, we will be recording this activity for coding by trained judges. For this activity, I would like for each of you to take some time and think about some recent positive event from your life. You are free to choose any event that comes to mind. It may be something that happened to you recently or in the past that continues to make you happy, something going on now, or something you anticipate will happen in the future. Examples of positive events would be receiving a good grade in class, a work promotion, being complimented by someone, being offered a job, being accepted into graduate school, etc. This event can be anything good (big or small) that has either happened recently or that you anticipate happening in the future. However, it must be something that has been on your mind lately, no matter big or small you may think it is.

You will take turns talking about describing your events to one another. When you are describing your event, try to go into as much detail as possible. When hearing about your partner's event you are free to talk as much or as little as you wish. In both situations, try to engage in a conversation that is as close as possible to a normal interaction you may have with a friend or someone you know. You will each have 5 minutes on the timer to complete this task. I will come in when the timer goes off. Do you have any questions? Now who will be the first to share the event?

\section{Post-Interaction/Post-Experiment Survey}

For the next part of the study, please complete this brief online survey. Make sure to read all of the instructions, and alert the research assistant when you are finished. Please respond as accurately as possible. As a reminder, your responses are completely confidential and will not be shared with your lab partner. 


\section{Debriefing Protocol}

Following the completion of the post-experiment questionnaires, participants were fully debriefed about the purpose of the study. Debriefing procedures were based on funneled debriefing methods developed by Bargh and Chartrand (2008). A research assistant verbally administered the following questionnaire to participants.

\section{Verbal Suspicion Questionnaire (Bargh \& Chartrand, 2000)}

1. What did you think the purpose of this study was?

2. What do you think this experiment was trying to study?

3. Did any aspect of this study seem strange or suspicious to you?

a. [If "yes"]: What part?

4. Did you think any of the tasks you did were related in any way?

a. [If "yes"]: In what way were they related?

5. Did anything you did on one task affect what you did on any other task?

a. [If "yes"]: How exactly did it affect you?

Participants who correctly guessed the purpose of the study (loneliness as influencing social itneractions), or who indicated that they think the 'self-reflection' activity affected what they did in the event-sharing activity were flagged, and eliminated from analyses. Bargh and Chartrand (2000) suggest excluding any participants whose answers to the debriefing questions are in the ballpark in regards to the purpose of the study and the link between the experimental manipulation (i.e., loneliness mood induction) and the experimental task (i.e., capitalization interaction).

Following completion of the Verbal Suspicion Questionnaire, participants were fully debriefed of the purpose of the study, and asked to not tell their peers about the purpose of the study or the specific activities completed during the lab session.

\section{Self-Affirmation Exercise}

Finally, we want to make sure that you leave the lab today feeling just as good as when you arrived. So, please take about 1-2 minutes to write down the most positive aspects of yourself on this notecard, which you can take home with you. When you are finished, you can grab your things and you are free to go.

[Hand participant list of resources and contact information as they leave the lab.] 